DEPARTMENT OF THE INTERIOR

\author{
U.S. GEOLOGICAL SURVEY \\ United States Earthquakes, 1978 \\ By \\ Carl A. Stover \\ and \\ Carl A. von Hake
}

Open-File report $84-978$

Prepared in cooperation with Nationa1 Oceanic and Atmospheric Administration. This report has not been reviewed for conformity with U.S. Geological Survey editorial standards. 



\section{Contributors}

Contributors to this publication are listed below according to the type of data supplied or service performed:

Hypocenters and magnitudes:

John S. Derr, U.S. Geological Survey

W. LeRoy Irby, U.S. Geological Survey

R. Kangas, U.S. Geological Survey

John H. Minsch, U.S. Geological Survey

Russ Needham, U.S. Geological Survey

Waverly J. Person, U.S. Geological Survey

Bruce W. Presgrave, U.S. Geological Survey

Hawaiian Volcano Observatory, U.S. Geological Survey

Intensities :

Carl w. Stover, U.S. Geological Survey

Network Operations (by institution):

University of Alaska

University of California, Berkeley

California Institute of Technology

Kansas Geological Survey

University of Oklahoma

Oklahoma Geological Survey

St. Louis University

U.S. Geological Survey

Virginia Polytechnic Institute and State University

Geodesy:

Sandford R. Holdahl, NOAA, NOS, National Geodetic Survey

Richard A. Snay, NOAA, NOS, National Geodetic Survey

William E. Strange, NOAA, NOS, National Geodetic Survey

Tsunamis :

Mark G. Spaeth, NOAA, National Weather Service

Strong-motion seismograph data:

Ronald L. Porcella, U.S. Geological survey

Editorial assistance and manuscript preparations:

Paula K. Smith, U.S. Geological Survey 



\section{Contents}

Page

Contributors........................................ $\ldots i$

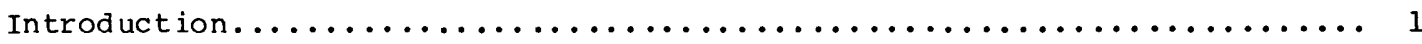

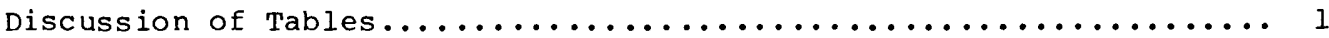

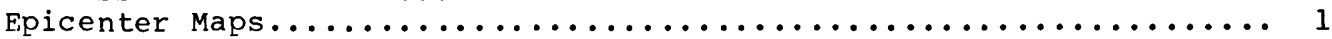

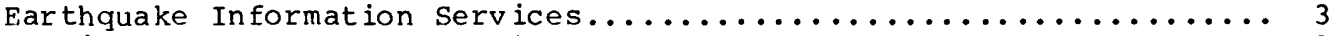

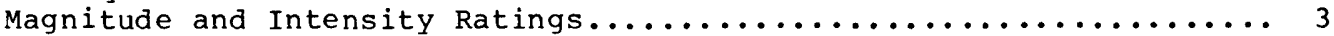

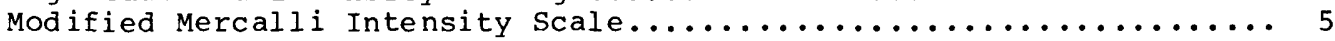

Acknowledgments....................................

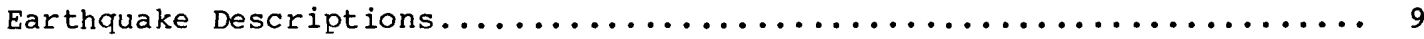

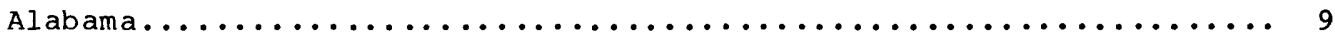

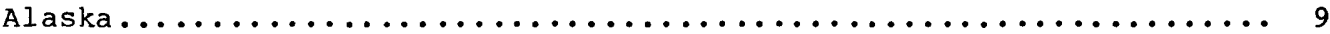

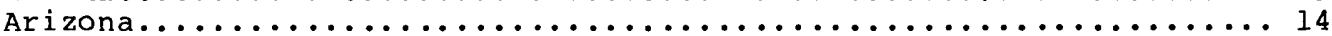

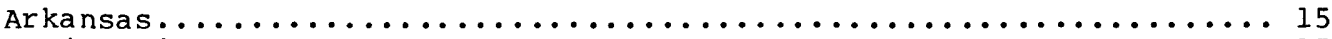

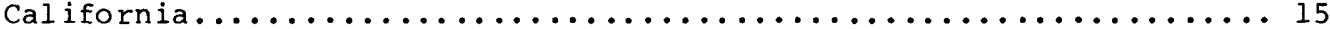

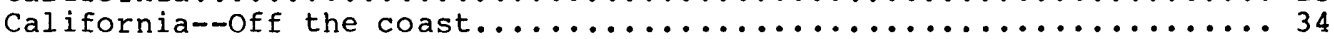

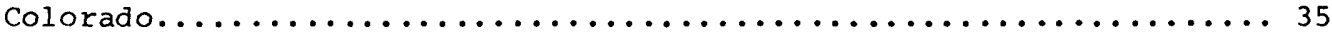

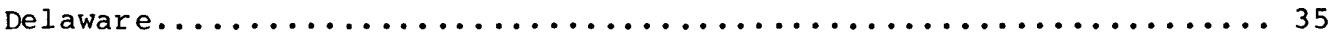

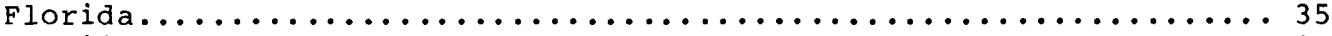

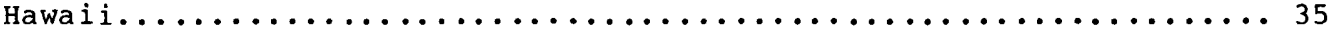

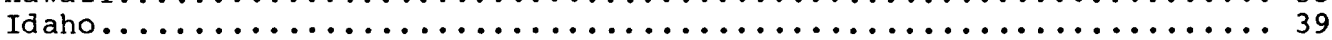

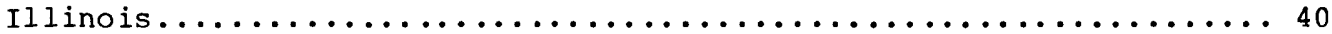

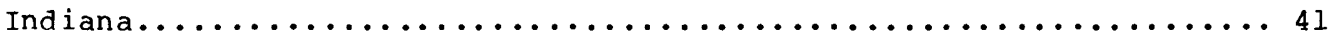

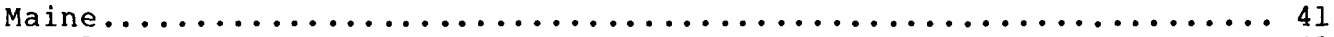

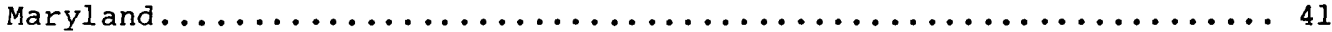

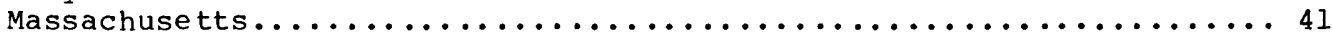

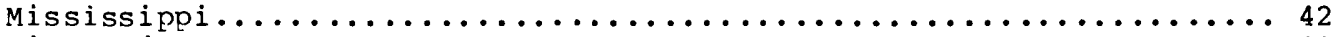

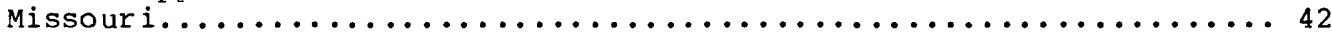

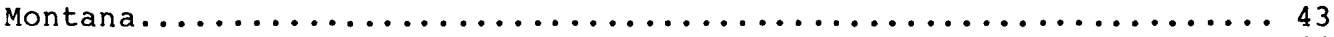

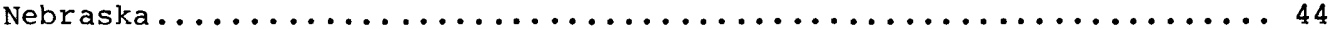

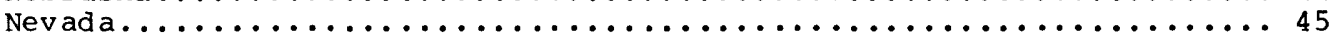

New Hampshire......................................... 46

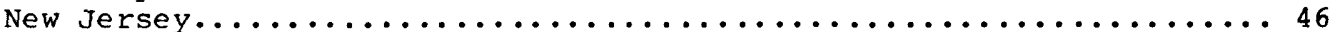

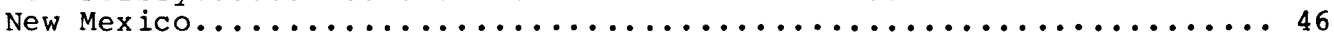

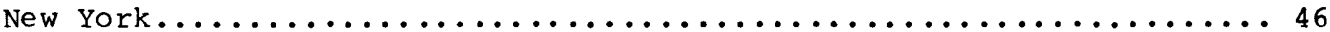

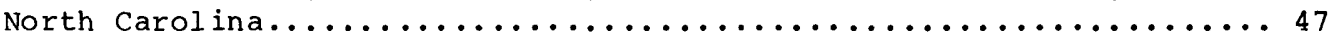

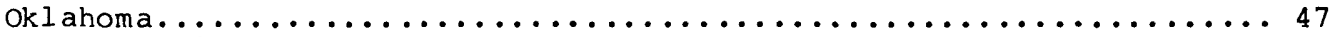

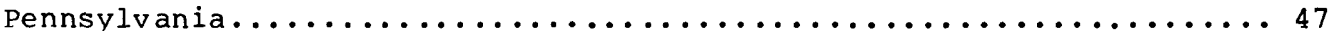

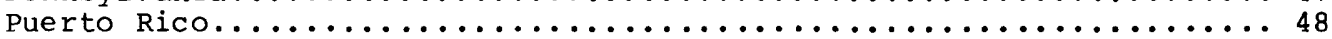

South Carolina....................................48

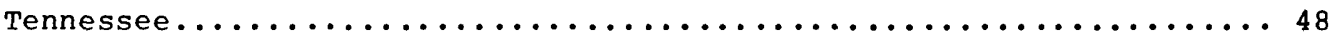

Texas.......................................... $4 . \ldots$

Utah.......................................... 49

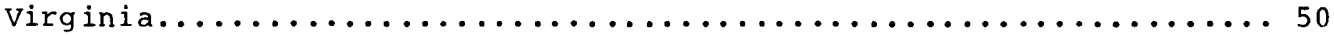

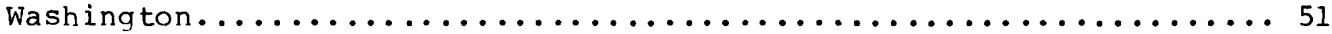

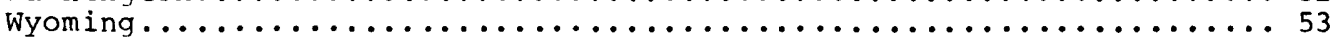

Network operations..................................... 82

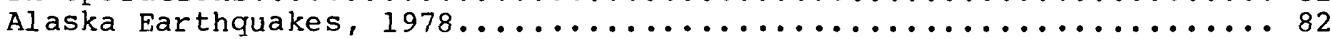
Northern and Central California Earthquakes, 1978.............. 82

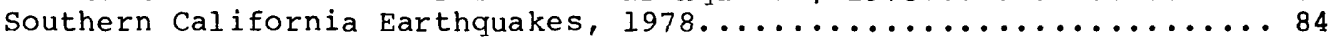
Central Mississippi Valley Earthquakes, 1978.................. 84

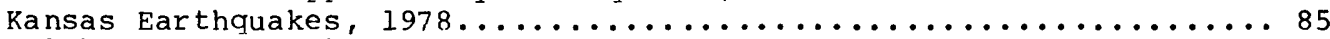

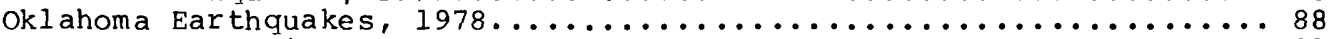

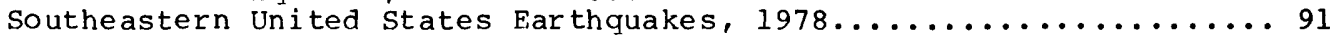

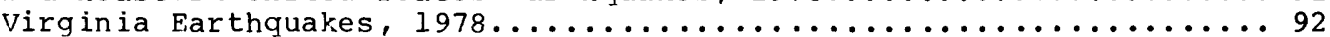




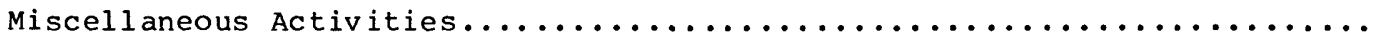

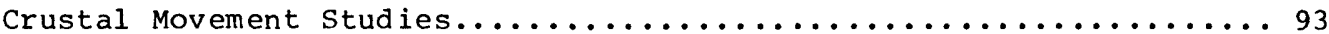

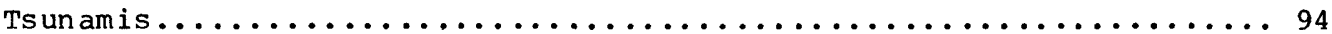

Principal Earthquakes of the World......................... 94

Strong-Motion Seismograph Data........................ 98

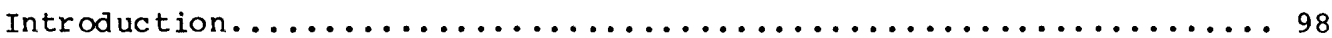

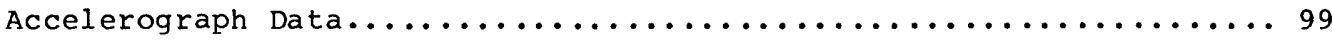

References......................................... 111

\section{LIST OF TABLES}

Table

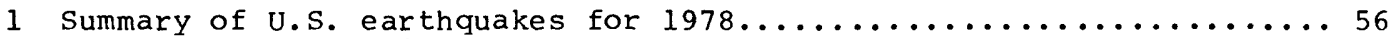

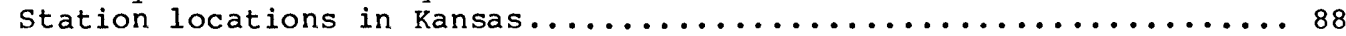

Microearthquake locations in Kansas for $1978 \ldots \ldots \ldots \ldots \ldots \ldots \ldots . \ldots . \ldots 8$

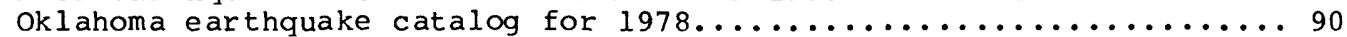

Southeastern United States earthquakes during $1978 \ldots \ldots \ldots \ldots \ldots \ldots 91$

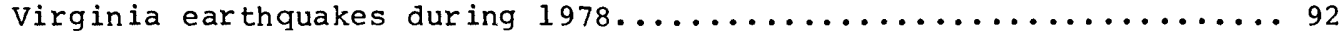

Principal earthquakes of the world during $1978 \ldots \ldots \ldots \ldots \ldots \ldots \ldots 95$

Summary of U.S. accel erograph records recovered during $1978 \ldots \ldots \ldots 104$

\section{LIST OF ILLUSTRATIONS}

Figure

Page

1 Summary of earthquake epicenters in the conterminous United States

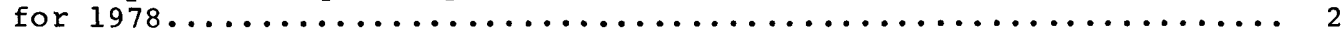

2 Summary of earthquake epicenters in Alaska for $1978 \ldots \ldots \ldots \ldots \ldots \ldots 2$

3 Summary of earthquake epicenters in Hawaii for $1978 \ldots \ldots \ldots \ldots \ldots . . .3$

4 Earthquakes plotted by Modified Mercalli Intensity in the conterminous United states for $1978 \ldots \ldots \ldots \ldots \ldots \ldots \ldots \ldots \ldots \ldots \ldots \ldots$

5 Earthquakes plotted by Modified Mercalli Intensity in Alaska for

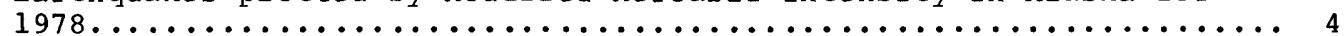

6 Earthquakes plotted by Modified Mercalli Intensity in Hawai for

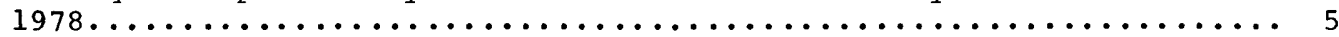

7 Isoseismal map for the northern California earthquake of 26 March

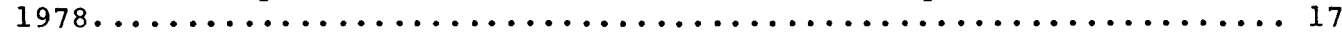

8 Isoseismal map for the southern California earthquake of 4 June 197820

9 Isoseismal map for the southern California earthquake of 5 June 197821

10 Photograph of Southern Pacific Transportation Company freight train

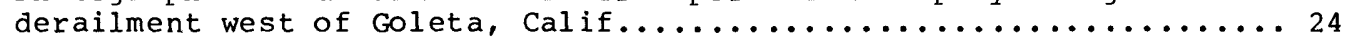

11 Isoseismal map for the southern California earthquake of 13 August

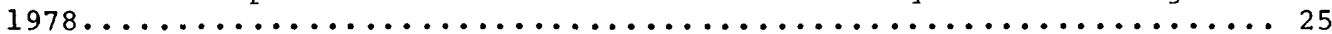

12 Photograph of damage to mobile home near Goleta, Calif........... 26

13 Isoseismal map for the Lake Tahoe, Calif, earthquake of 4 september

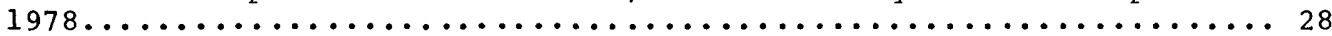

14 Isoseismal map for the northern California earthquake of 8 September

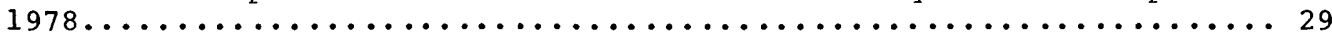

15 Isoseismal map for the Owens Valley, California earthquake of

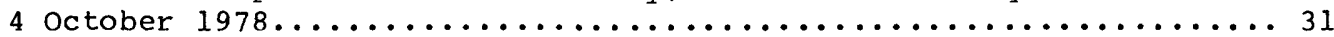

16 Isoseismal map for the central Idaho earthquake of 29 october $1978 . .40$

17 Isoseismal map for the southeastern Idaho earthquake of 30 November

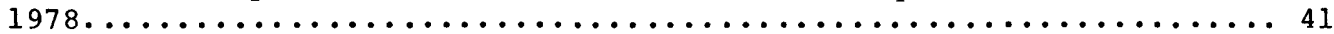

18 Isoseismal map for the southern Illinois earthquake of 5 December

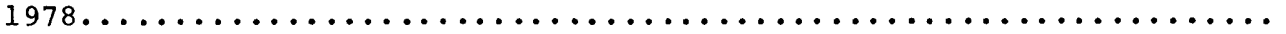

19 Isoseismal map for the eastern Missouri earthquake of 20 September

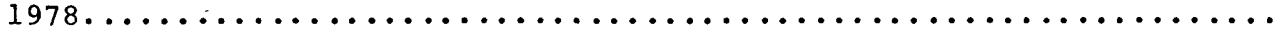


20 Isoseismal map for the western Montana earthquake of 23 Apri1 1978.. 44

21 Isoseismal map for the southeastern Pennsylvania earthquake of

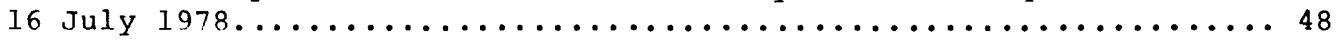

22 Isoseismal map for the west Texas earthquake of 16 June $1978 \ldots \ldots \ldots$

23 Isoseismal map for the puget Sound, Washington earthquake of

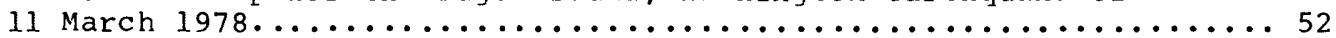

24 Isoseismal map for the puget Sound, Washington earthquake of

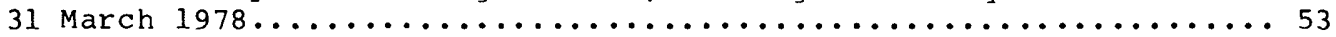

25 Isoseismal map for the Seattle, Washington earthquake of 31 December

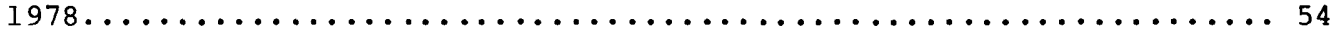

26 Northern California earthquakes during $1978 \ldots \ldots \ldots \ldots \ldots \ldots$

27 Plot of the cumulative number of earthquakes versus 1 ocal Richter

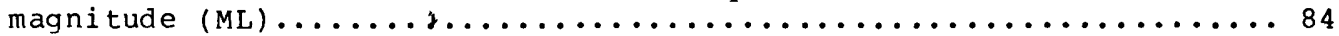

28 Southern California earthquakes of magnitude $2.5 \mathrm{ML}$ or greater

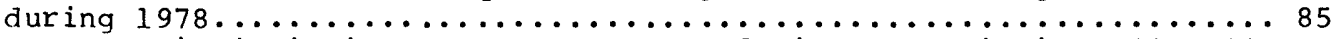

29 Central Mississippi valley earthquakes during 1978 within a $4^{\circ} \times 4^{\circ}$

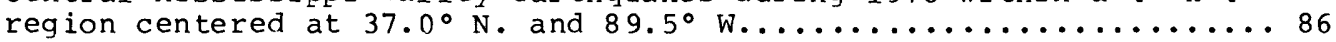

30 Central Mississippi Valley earthquakes during 1978 within a $1.5^{\circ} \mathrm{x}$ $1.5^{\circ}$ region centered at $36.25^{\circ} \mathrm{N}$. and $89.75^{\circ} \mathrm{w} \ldots \ldots \ldots \ldots \ldots$

31 Kansas Geological Survey seismograph network in 1978............87

32 Kansas earthquakes and the Humboldt Fault Zone................. 87

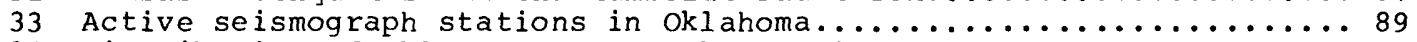

34 Distribution of oklahoma earthquakes during 1978.............. 89

35 Southeastern United States earthquake epicenters during 1978...... 91

36 Southeastern United States seismic stations operating in $1978 \ldots \ldots . \ldots 91$

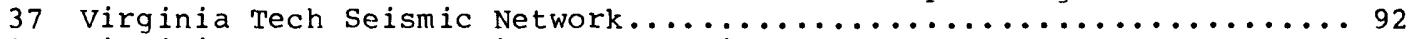

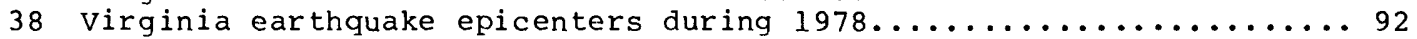

39 Coyote Dam accelerograms from the Ukiah, Calif. earthquake of

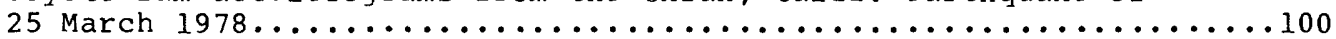

40 Location map, Santa Barbara, Calif. earthquake of 13 August 1978...101

41 Accelerograms from Santa Barbara Courthouse and Cachuma Dam, Santa

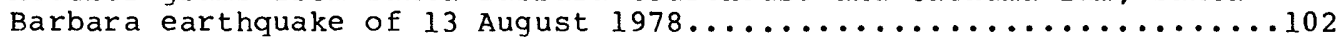

42 Selection of Monticello Dam accelerograms from the Jenkinsville, South Carolina earthquake of February to November 1978..........103 


\title{
United States Earthquakes, 1978
}

\author{
Carl W. Stover, U.S. Geological Survey \\ Carl A. von Hake, NOAA, Environmental Data and Information Service
}

\section{Introduction}

This publication describes all earthquakes that were reported felt in the United States and nearby territories in 1978. It has been compiled by the U.S. Geological Survey (USGS) and published jointly with NOAA, Environmental Data and Information Service (EDIS). Its purpose is to provide a continuous history of U.S. earthquakes for studying seismic risk, evaluating nuclear powerplant sites, designing earthquake-resistive structures, and answering inquiries from the scientific and general public.

The publication is composed of four major chapters: "Earthquake Descriptions," which includes a summary of macroseismic data reported for each earthquake and a chronological list of earthquakes by State (table 1); "Network Operations," which summarizes the results from local seismic networks; "Miscellaneous Activities," which contains information on crustal movement studies, tsunamis, and principal earthquakes of the world (table $7)$, and "Strong-Motion Seismograph Data" (table 8). The intensity and macroseismic data in "Earthquake Descriptions" are compiled from questionnaire canvasses (see next paragraph), newspaper articles, and reports prepared by other government organizations, state institutions, local organizations, and individuals. Each description includes date, origin time, hypocenter, and hypocenter source of the earthquake, maximum intensity (Modified Mercalli), and macroseismic effects reported in the area.

The USGS collects macroseismic intensity information primarily by mailing questionnaires, "Earthquake Report" forms, to postmasters in the earthquake area. postmasters complete the forms and return them to the USGS, where they are evaluated and intensities are assigned. For damaging earthquakes, the questionnaires are supplemented by USGS field investigations. The USGS publishes preliminary intensity data in its quarterly Earthquakes in the United states. The final information is published in the United states Earthquakes series, issued annually since $1 \overline{928}$.
7 include date, origin time, hypocenter (epicenter and focal depth), and magnitude. Table 1 also contains the Modified Mercalli (MM) intensity.. The origin time and date are listed in Universal Coordinated Time (UTC). The epicenters were taken principally from the USGS Preliminary Determination of Epicenters, Monthly Listings or Earthquakes in the United states ${ }^{2}$. The $\frac{\text { accuracy of }}{\text { the }}$ epicenters is that claimed by the institution supplying the hypocenter and is not necessarily the accuracy indicated by the number of decimals listed. The epicenters located by the USGS have a varying degree of accuracy, usually twotenths of a degree or less, depending on their continental or oceanic location. The oceanic hypocenters are less accurate than those on the continent, even though both are listed to two decimals. Depths are 1 isted to the nearest whole kilometer.

Magnitude values 1 isted in the tables were furnished by cooperating institutions or determined by the USGS. The computational sources are labeled according to the assigned letter codes shown in headnotes to the tables.

\section{EPICENTER MAPS}

Figures $1-3$ are computer plots of all earthquake epicenters in the conterminous United States, Alaska, and Hawaii listed in table 1. Each earthquake is indicated by a small circle.

Figures 4-6 are computer plots of 1978 earthquakes in the conterminous United States, Alaska, and Hawai $i$ by Modified Mercalli (MM) intensity. Maximum intensities are represented by Arabic numerals at the location that each occurred. Earthquakes of intensity I-IV are represented by dots.

\footnotetext{
${ }^{1}$ For sale by the superintendent of Documents, U.S. Government Printing Office, Washington, D.C. 20402 .

${ }^{2}$ Copies of these reports are available free on application to Branch of Distribution, U.S. Geological Survey, 1200 South
} Eads Street, Arlington, Va. 22202 . 


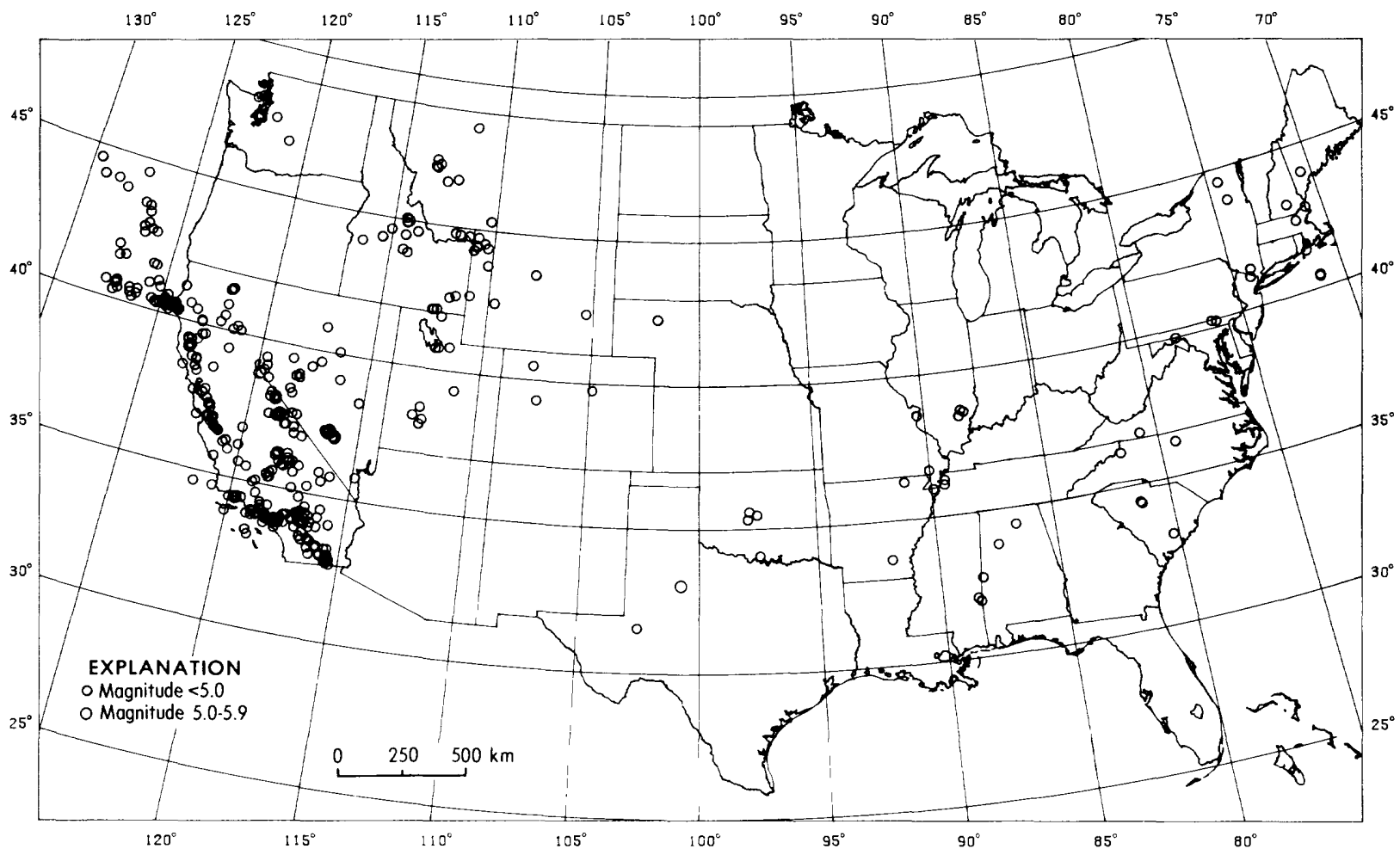

FIGURE 1.-- Summary of earthquake epicenters in the conterminous United states for 1978 .

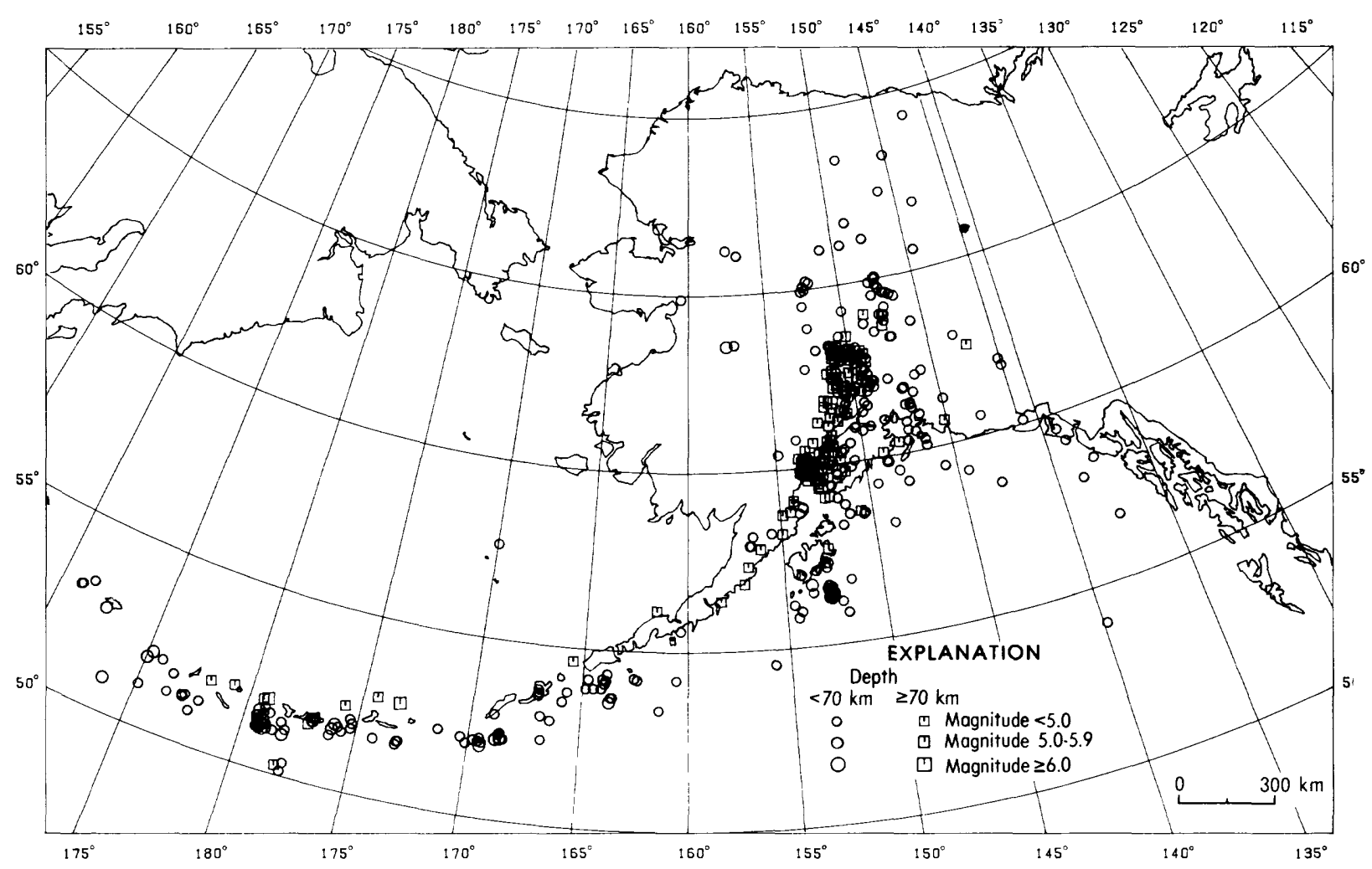

FIGURE 2.--Summary of earthquake epicenters in Alaska for 1978. 


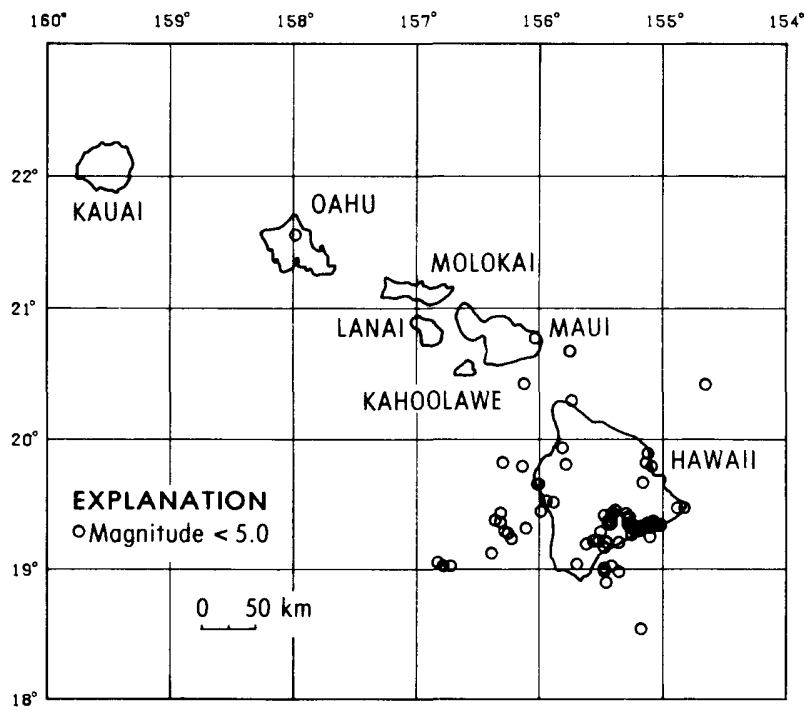

FIGURE 3.--summary of earthquake epicenters in Hawaii for 1978.

The selection of intensity or isoseismal maps shown in the Earthquake Descriptions is governed largely by the size of the area affected. This means that sharp, localized shocks of intensity VI (which occur mostly in California) may not be represented by these maps, whereas other earthquakes of intensity $V$ and VI (which occur largely in the Eastern and Central States) often will be illustrated because of the larger felt areas. Numerals on these computer-plotted maps represent the maximum MM intensities at each town. Isoseismal contours are a generalization of intensity data and are extrapolated to regions that have no observations. The contours do not include each intensity observation.

\section{EARTHQUAKE INFORMATION SERVICES}

The USGS coordinates the collection of all types of earthquake information, with the special objective of correlating instrumentally determined earthquake locations with noninstrumental locations indicated by intensity data. This correlation is achieved through intensive regional investigations of earthquakes by local organizations and the USGS. Primary data are gathered by a canvass of the epicentral area using questionnaire cards. When returned and analyzed, this information is used to map the seismic areas of the country in order to promote public safety through a better understanding of earthquake phenomena.

The National Geophysical and SolarTerrestrial Data Center (NGSDC), one of the five major facilities of NOA's Environmental Data and Information Service, is responsible for data activities in seismology. Its services include preparing local and regional seismic histories for engineers, actuaries, and other scientists and answering direct inquiries from the public on all aspects of historical earthquakes. Additional services and products include publishing annual earthquake summaries and revised historical earthquake reports; and making available copies of seismograms, accelerograms, displacement meter records, digitized strong-motion seismograms, and epicenter lists in several formats. Many of these products and services are based on seismic records or other data that have originated with USGS recording networks or with USGS data-reduction facilities. Information concerning services and products of NGSDC may be obtained from the National Geophysical and Solar-Terrestrial Data Center, NOAA/EDIS, Boulder, CO 80303.

\section{MAGNITUDE AND INTENSITY RATINGS}

Magnitude, a measure of the "size" of an earthquake, is roughly related to the energy release at the focus of an earthquake. Although the magnitude scale has neither "top" nor "bottom" values the highest ever recorded was magnitude 8.9 and the lowest about -3 . On this logarithmic scale, a magnitude 6 shallow-focus earthquake represents elastic-wave energy about 30 times greater than that generated by a magnitude 5 earthquake, 900 times greater than that of a magnitude 4 shock, and so forth. Many factors enter into the determination of earthquake magnitude, including earthquake focal depth, frequency content of the sampled energy, and the earthquake radiation pattern. Magnitude values calculated by the USGS are based on the following formulas:

$$
M S=\log (\mathrm{A} / \mathrm{T})+1.66 \log \mathrm{D}+3.3 .
$$

as adopted by the International Association of Seismology and Physics of the Earth's Interior (IASPEI; Bath, 1966, p. 153), where $A$ is the maximum vertical surface-wave ground amplitude, in micrometers; $T$ is the period, in seconds, and $18<\mathrm{T}<22$; and $\mathrm{D}$ is the distance in geocentric degrees (station to epicenter), and $20^{\circ}<\mathrm{D}<160^{\circ}$. No depth correction is made for depth less than $50 \mathrm{~km}$, and no MS magnitudes are computed for depths greater than $50 \mathrm{~km}$.

$$
m b=\log (A / T)+Q(D, h),
$$

as defined by Gutenberg and Richter (1956), except that $T$, the period in seconds, is restricted to $0.1<\mathrm{T}<3.0$, "and $A$, the ground amplitude in micrometers, is not necessarily the maximum of the P-wave group. $O$ is a function of distance $D$ and depth $h$, where $D \geq 5^{\circ}$.

$$
M L=\log A-\log A_{0},
$$




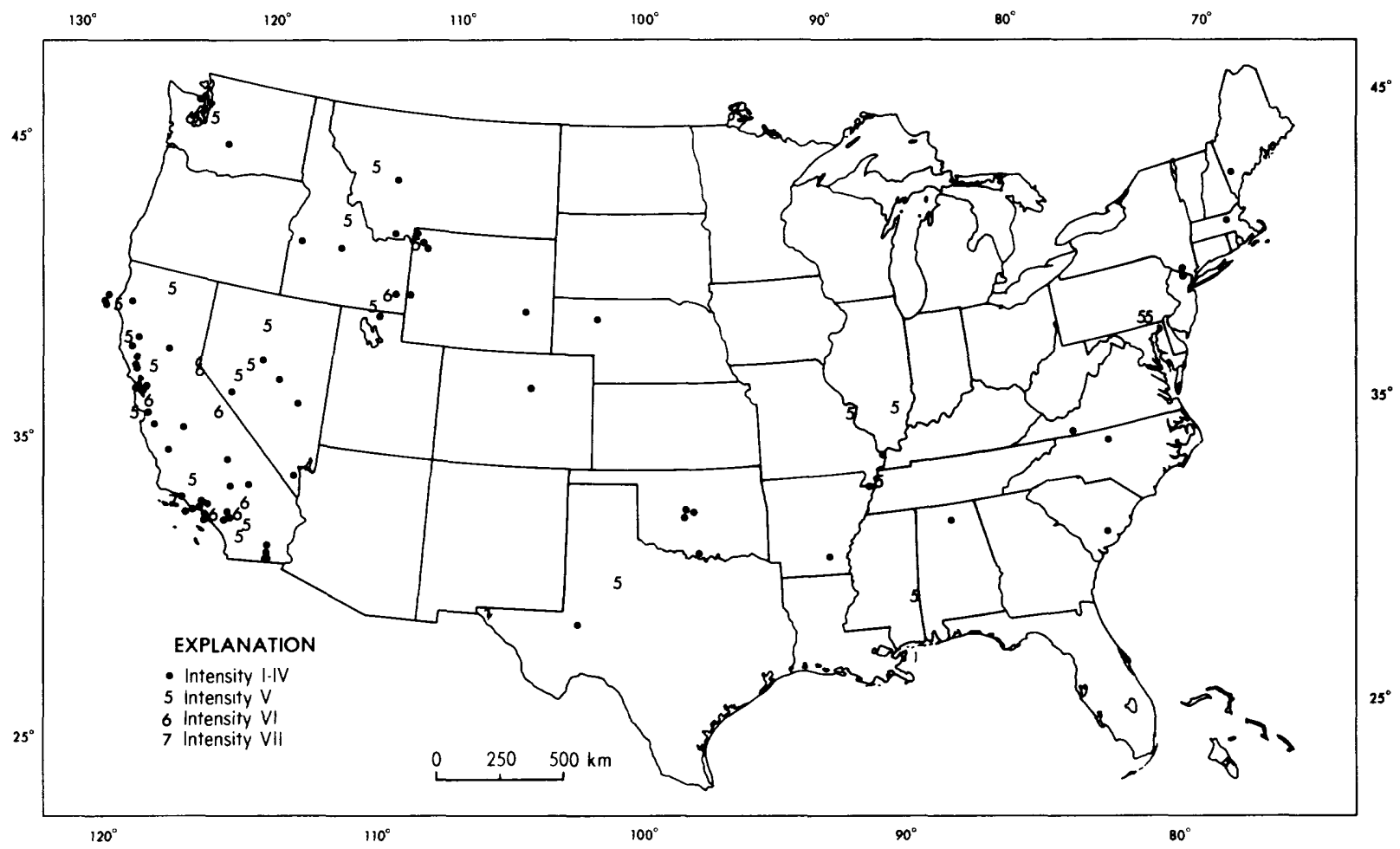

Figure 4.--Earthquakes plotted by Modified Mercalli Intensity in the conterminous United States for 1978 .

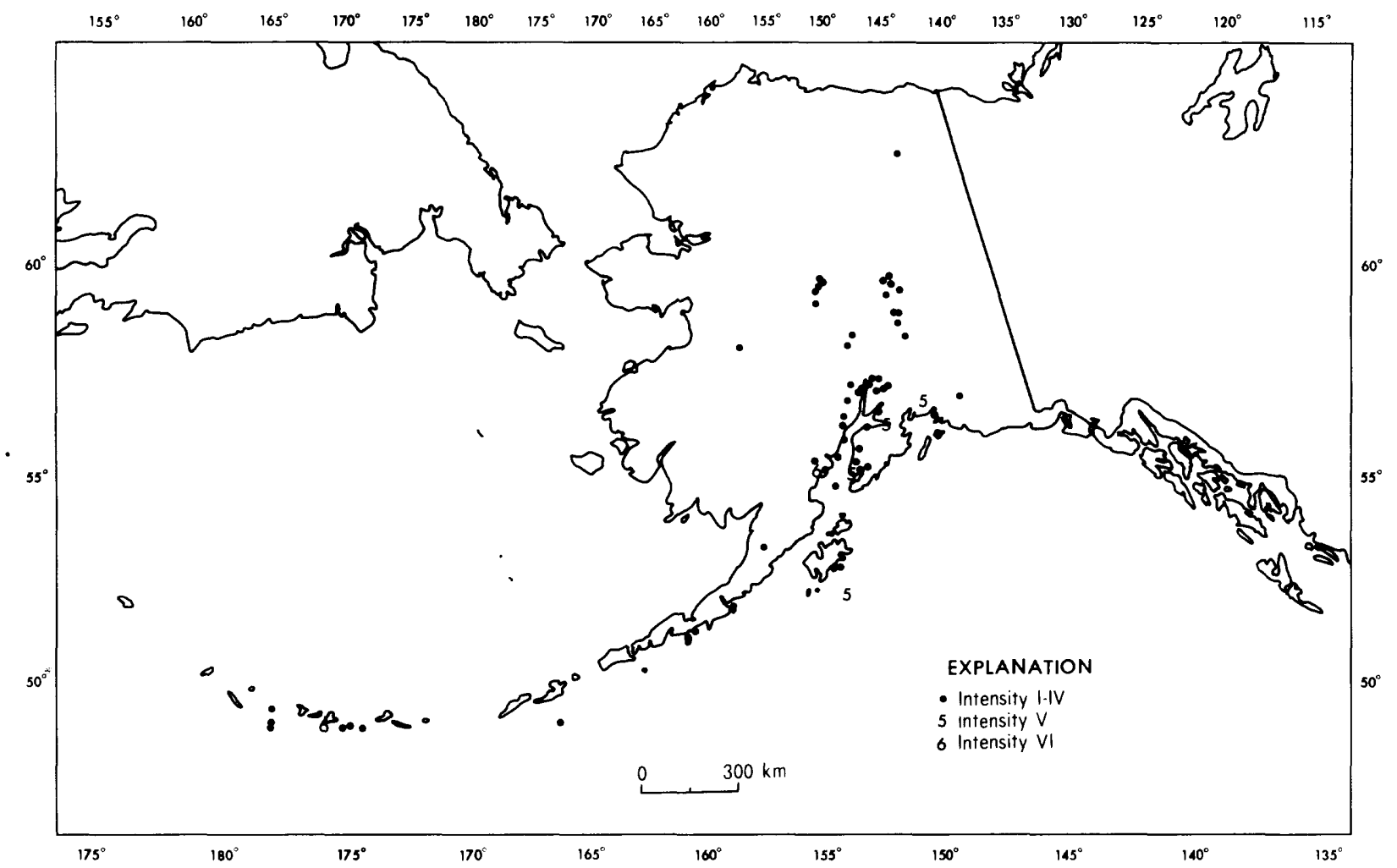

Figure 5.--Earthquakes plotted by Modified Mercalli Intensity in Alaska for 1978. 


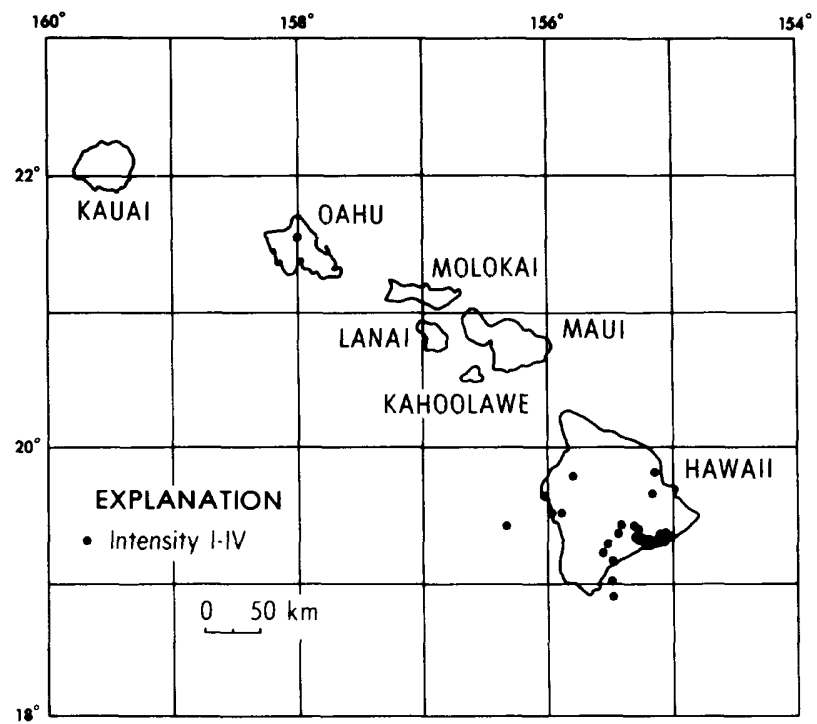

Figure 6.--Earthquakes plotted by Modified Mercalli Intensity in Hawaii for 1978 .

where $A$ is the maximum trace amplitude in millimeters, written by a wood-Anderson torsion seismometer, and $\log A_{0}$ is a standard value as a function of distance, where the distance is $<600 \mathrm{~km}$. ML values are also calculated from other seismometers by conversion of recorded ground motion to the expected response of the torsion seismometer:

$$
\begin{gathered}
\mathrm{mbLg}=3.75+0.90(\log \mathrm{D}) \\
+\log (\mathrm{A} / \mathrm{T}) 0.5^{\circ} \leq \mathrm{D} \leq 4^{\circ} \\
\mathrm{mbLg}=3.30+1.66(\log \mathrm{D}) \\
+\log (\mathrm{A} / \mathrm{T}) 4^{\circ} \leq \mathrm{D} \leq 30^{\circ}
\end{gathered}
$$

as proposed by Nuttli (1973), where A/T. is expressed in micrometers per second, calculated from the vertical-component lsecond $\mathrm{Lg}$ waves, and $\mathrm{D}$ is the distance in geocentric degrees.

Intensity, as applied to earthquakes, represents a quantity determined from the effects on people, manmade objects, and the Earth's surface (landslides, ground fissures). Intensities are assigned according to the descriptions 1 isted in the Modified Mercalli Intensity Scale of 1931 (Wood and Neumann, 1931). There are 12 discrete steps in the MM scale (see next section). An earthquake in a populated area will have different intensities at different localities, owing to the distance from the epicenter of the earthquake, local geological conditions, structural design of buildings, and the earthquake magnitude.

The text of this publication gives the intensity for each city where the earthquake was felt and summaries of the strongest effects reported. Each earthquake is further characterized by its max- imum intensity, which is given in the text and in table 1 .

Although the Modified Mercalli Intensity Scale is in many instances in adequate for present-day requirements, the scale has been the guide used by the USGS and NOAA and will continue to be so used until a new scale has been devised and has acceptance in the engineering and seismological communities. Questions concerning the interpretation of historical earthquake intensities should be referred to the USGS.

\section{MODIFIED MERCALLI INTENSITY}

\section{SCALE OF 1931}

E. 'apted from Sieberg's Mercalli-Cancani scale, modified and condensed.

I. Not felt - or, except rarely under especially favorable circumstances. Under certain conditions, at and outside the boundary of the area in which a great shock is felt: sometimes birds, animals, reported uneasy or disturbed; sometimes dizziness or nausea experienced; sometimes trees, structures, 1 iquids, bodies of water, may sway--doors may swing, very slowly.

II. Felt indoors by few, especially on upper floors, or by sensitive, or nervous persons. Also, as in grade $I$, but often more noticeably: sometimes hanging objects may swing, especially when delicately suspended; sometimes trees, structures, liquids, bodies of water, may sway, doors may swing, very slowly; sometimes birds, animals, reported uneasy or disturbed; sometimes dizziness or nausea experienced.

III. Felt indoors by several, motion usually rapid vibration. Sometimes not recognized to be an earthquake at first. Duration estimated in some cases. Vibration like that due to passing of light, or lightly loaded trucks, or heavy trucks some distance away. Hanging objects may swing slightly. Movements may be appreciable on upper levels of tall structures. Rocked standing motor cars slightly.

IV. Felt indoors by many, outdoors by few. Awakened few, especially light sleepers. Frightened no one, unless apprehensive from previous experience. Vibration like that due to passing of heavy or heavily loaded trucks. Sensation like heavy body striking building or falling of heavy 
objects inside. Rattling of dishes, windows, doors; glassware and crockery clink and clash. Creaking of walls, frame, especially in the upper range of this grade. Hanging objects swung, in numerous instances. Disturbed liquids in open vessels slightly. Rocked standing motor cars noticeably.

V. Felt inaoors by practically all, outdoors by many or most: outdoors direction estimated. Awakened many, or most. Erightened few--slight excitement, a few ran outdoors. Buildings trembled throughout. Broke dishes, glassware, to some extent. Cracked windows--in some cases, but not generally. Overturned vases, small or unstable objects, in many instances, with occasional fall. Hanging objects, doors, swing generally or considerably. Knocked pictures against walls, or swung them out of place. Opened, or closed, doors, shutters, abruptly. Pendulum clocks stopped, started or ran fast, or slow. Moved small objects, furnishings, the latter to slight extent. Spilled liquids in small amounts from well-filled open containers. Trees, bushes, shaken slightly.

VI. Felt by all, indoors and outdoors. Frightened many, excitement general, some alarm, many $r$ an outdoors. Awakened all. Persons made to move unsteadily. Trees, bushes, shaken slightly to moderately. Liquid set in strong motion. Small bells rang--church, chapel, school, etc. Damage slight in poorly built buildings. Fall of plaster in small amount. Cracked plaster somewhat, especially fine cracks chimneys in some instances. Broke dishes, glassware, in considerable quantity, also some windows. Fall of knick-knacks, books, pictures. Overturned furniture in many instances. Moved furnishings of moderately heavy kind.

VII. Frightened all--general al arm, all ran outdoors. Some, or many, found it difficult to stand. Noticed by persons driving motor cars. Trees and bushes shaken moderately to strongly. Waves on ponds, lakes, and runnina water. Water turbid from mud stirred up. Incaving to some extent of sand or gravel stream banks. Rang large church bells, etc. Suspended objects made to quiver. Damage negligible in buildings of good design and construction, slight to moderate in wellbuilt ordinary buildings, considerable in poorly built or badly designed buildings, adobe houses, old walls (especially where laid up without mortar), spires, etc. Cracked chimneys to considerable extent, walls to some extent. Fall of plaster in considerable to large amount, also some stucco. Broke numerous windows, furniture to some extent. Shook down loosened brickwork and tiles. Broke weak chimneys at the roof-line (sometimes damaging roofs). Fall of cornices from towers and high buildings. and stones. Dislodged furniture, with damage from breaking. Damage considerable to concrete irrigation ditches.

VIII. Fright general--alarm approaches panic. Disturbed persons driving motor cars. Trees shaken strongly--branches, trunks, broken off, especially palm trees. Ejected sand and mud in small amounts. Changes: temporary, permanent; in flow of springs and wells; dry wells renewed flow; in temperature of spring and well waters. Damage slight in structures (brick) built especially to withstand earthquakes. Considerable in ordinary substantial buildings, partial collapse: racked, tumbled down, wooden houses in some cases; threw out panel walls in frame structures, broke off decayed piling. Fall of walls. Cracked, broke, solid stone walls seriously. Wet ground to some extent, also ground on steep slopes. Twisting, fall, of chimneys, columns, monuments, also factory stacks, towers. Moved conspicuously, overturned, very heavy furniture.

IX. Panic general. Cracked ground conspicuously. Damage considerable in (masonry) structures built especially to withstand earthquakes: Threw out of plumb some wood-frame houses built especially to withstand earthquakes; great in substantial (masonry) buildings, some collapse in large part; or wholly shifted frame buildings oft foundations, racked frames; serious to reservoirs; underground pipes sometimes broken.

X. Cracked ground, especially when loose and wet, up to widths of several inches; fissures up to 
a yard in width ran parallel to canal and stream banks. Landslides considerable from river banks and steep coasts. Shifted sand and mud horizontally on beaches and flat $l$ and. Changed level of water in wells. Threw water on banks of canals, lakes, rivers, etc. Damage serious to dams, dikes, embankments. Severe to well-built wooden structures and bridges, some destroyed. Developed dangerous cracks in excellent brick walls. Destroyed most masonry and frame structures, also their foundations. Bent railroad rails slightly. Tore apart, or crushed endwise, pipe lines buried in earth. Open cracks and broad wavy folds in cement pavements and asphalt road surfaces.

$X I$. Disturbances in ground many and widespread, varying with ground material. Broad fissures, earth slumps, and land slips in soft, wet ground. Ejected water in large amounts charged with sand and mud. Caused sea-waves ("tidal" waves) of significant magnitude. Damage severe to wood-frame structures, especially near shock centers. Great to dams, dikes, embankments often for long distances. Few, if any (masonry) structures remained standing. Destroyed large well-built bridges by the wrecking of supporting piers, or pillars. Affected yielding wooden bridges less. Bent railroad rails greatly, and thrust them endwise. Put pipe lines buried in earth completely out of service.

XII. Damage total--practically all works of construction damaged greatly or destroyed. Disturbances in ground great and varied, numerous shearing cracks. Landslides, falls of rock of significant character, slumping of river banks, etc., numerous and extensive. Wrenched loose, tore off, large rock masses. Fault slips in firm rock, with notable horizontal and vertical offset displacements. Water channels, surface and underground, disturbed and modified greatly. Dammed lakes, produced waterfalls, deflected rivers, etc. Waves seen on ground surfaces (actually seen, probably, in some cases). Distorted lines of sight and level. Threw objects upward into the air.

\section{ACKNOWLEDGMENTS}

Active cooperation in earthquake investigations in the united States is provided by several seismology collaborators. The following served as collaborators to the USGS and NOAA during 1978.

Alabama.--L. J. Eisele, Spring Hill College, Mobile.

Alaska.--Staff of NOAA-Alaska Tsunami Warning Center, Palmer, and $\mathrm{J}$. B. Townshend, College Observatory, College.

Arizona.--Marc Sbar, University of Arizona, Tucson.

California (northern).--Bruce A. Bolt, University of California, Berkeley.

California (southern).--Clarence R. Allen, California Institute of Technology, Pasadena.

Connecticut.--Robert Miller, University of Connecticut, Groton.

Delaware.--Kenneth D. Woodruff, University of Delaware, Newark.

Florida and Georgia.--Leland T. Long, Georgia Institute of Technology, Atlanta.

Hawaii.--Robert Koyanagi, USGS, Hawaiian Volcano Observatory, Hawaii National Park.

Idaho.--James K. Applegate, Boise State University, Boise.

Indiana.--Robert F. Blakely, Department of Natural Resources, Geological Survey, Bloomington.

Iowa.--J. P. Kopp, Loras College, Dubuque.

Kansas.-Henry V. Beck, Kansas State University, Manhattan, and Don $W$. Steeples, Kansas Geological Survey, Lawrence.

Kentucky.--Ronald L. Street, University of Kentucky, Lexington.

Michigan.--F. Mauk, University of Michigan, Ann Arbor.

Minnesota.--Harold Mooney, University of Minnesota, Minneapol is.

Missouri, Illinois, Arkansas area.--ot to Nuttli and Robert B. Hermann, Saint Louis University, Saint Louis.

Montana.--Anthony Oamar, University of Montana, Missoula.

New England.--E. Chiburis, Boston College, Weston, Mass.

New York.--Lynn R. Sykes and Yash P. Aggarwal, Lamont-Doherty Geological observatory, Pal isades.

Ohio.--Edward J. Walter, John Carroll University, Cleveland.

Oklahoma.--James E. Lawson, Jr., Oklahoma Geological Survey, Leonard.

Oregon.--Richard W. Couch, Oregon University, Corvallis.

Pennsylvania.--Benjamin F. Howell, Jr., Pennsylvania State University, University Park.

South Carolina.--Pradeep Talwani, University of South Carolina, Columbia, and 
Joyce Bagwell, Baptist College of Charleston, Charleston.

Tennessee.--Berlen C. Moneymaker, Knoxville.

Texas.--G. R. Keller, University of Texas, El Paso.

Utah.--Kenneth Cook, University of Utah, Salt Lake City.

Virginia.--G. A. Bollinger, Virginia
Polytechnic Institute and state University, Blacksburg.

Washington.--Norman Rasmussen and Robert S. Crosson, University of Washington, Seattle.

West Virginia.--R. W. Laird, West Virgina University, Morgantown.

wisconsin.--David E. Willis, University of wisconsin, Milwaukee. 


\section{Earthquake Descriptions}

This section lists all earthquakes alphabetically by state. The origin time of earthquake occurrences is given in Universal Coordinated Time (UTC). Times are expressed continuously from midnight to midnight, or 0 to 24 hours.

Sources of noninstrumental information (macroseismic data) in this publication include questionnaire canvasses conducted by the USGS; newspaper articles; bulletins of the seismological society of America; and special earthquake reports of other organizations. Instrumental data are provided by the USGS, National Earthquake Information Service.

Roman numerals in the earthquake descriptions refer to the Modified Mercalli Intensity Scale of 1931 (see page 2), which gives about equal weight to the disturbance of inanimate objects and to personal reactions. When more than one degree of intensity is reported from a town, the town is assigned the highest intensity reported. All earthquake reports that contain minimal information are assigned intensity II.

[The following symbols are used to indicate authority for arrival or origin times, epicenters, and/or magnitudes: (A) Geophysical Institute, University of Alaska, Fairbanks; (B) University of California, Berkeley; (D) University of Montana, Missoula; (E) U.S. Department of Energy, Las Vegas, Nevada; (G) U.S. Geological Survey, National Earthquake Information Service, Golden, Colo.; (H) U.S. Geological Survey, Hawaiian Volcano Observatory, Hawaii National Park; (J) Weston Observatory, Vieston, Mass.; (K) Lee, W. H. $\mathrm{K}$. and others, 1978; (L) Lamont-Doherty Geological Observatory, Palisades, N.Y.; (M) NOAA, Alaska Tsunami Warning Center, Palmer; (O) Earth Physics Brarch, Ottawa, Canada; (P) California Institute of Technology, Pasadena; (Q) Pacific Geoscience Centre, Sydney, R.C., Canada; (S) St. Louis University, St. Louis, Mo.; (T) University of oklahoma, Leonard; (U) University of Utah, Salt Lake City; (V) Virginia Polytechnic Institute and state University, Blacksburg; (W) University of Washington, Seattle]

\section{Alabama}

1 March (G) Northern Alabama Origin time: $0408 \quad 26.6$ Epicenter: $34.42 \mathrm{~N} .86 .61 \mathrm{~W}$.
Alabama--Continued

$\begin{array}{ll}\text { Depth: } & 5 \mathrm{~km} \\ \text { Magnitude: } & 2.5 \mathrm{mbLg} \\ \text { Intensity III: } & \text { Huntsville area (V). }\end{array}$

11 December (G) Alabama-Mississippi border Origin time: $\begin{array}{r}\text { area } \\ 0206\end{array} \quad 48.2$

See Mississippi listing.

Alaska

5 January (G) Southern Alaska

Origin time: $\quad 195609.8$

Epicenter: $\quad 61.33 \mathrm{~N}, 151.65 \mathrm{~W}$.

Depth :

Magnitude: $110 \mathrm{~km}$

Intensity III: Anchorage (M), Wasilla (M).

Intensity II: Palmer (M).

6 January (G) Andreanof Islands, Aleutian Origin time: 070843.8

Epicenter: $\quad 51.78 \mathrm{~N} .176 .01 \mathrm{~W}$.

Depth:

Magnitude:

$63 \mathrm{~km}$

Intensity IV: Adak (M).

6 January (G) Kenai Peninsula

origin time: 215901.1

Epicenter: $\quad 60.91 \mathrm{~N} ., 149.38 \mathrm{~W}$.

Depth:

Magnitude: $\quad 4.6 \mathrm{mb}, 4.9 \mathrm{MI}(\mathrm{M})$

Intensity $V:$ Girdwood (small

objects fell, not broken).

Intensity IV: Anchorage, Chugiak, Clam Gulch, Cooper Landing, Fagle River, Kenai, Ouzinkie, Seward, Soldotna, Whittier.

Intensity III: Elmendorf AFB, Moose Pass, Palmer.

9 January (G) Southern Alaska origin time: 070605.8

Epicenter: $\quad 62.00 \mathrm{~N}, 148.82 \mathrm{~W}$.

Depth:

Magnitude: $\quad 3.5 \mathrm{ML}(\mathrm{M})$

Intensity III: Anchorage, Lower

Susitna Valley, Matanuska.

9 January (G) Andreanof Islands, Aleutian Islands

Origin time: $\quad 221814.6$

Epicenter: $\quad 51.61 \mathrm{~N} .177 .17 \mathrm{~W}$.

Depth :

$121 \mathrm{~km}$

Magnitude:

$3.9 \mathrm{mb}$

Intensity II: Adak (M). 
Alaska--Continued

10 January (A) Central Alaska

Origin time: $\quad 1209 \quad 16.4$

Epicenter: $\quad 64.74 \mathrm{~N} ., 147.44 \mathrm{~W}$.

Depth:

Magnitude:

$24 \mathrm{~km}$

Intensity

18 January (G) Fox Islands, Aleutian Islands Islands

origin time: $\quad 170418.1$

Epicenter: $\quad 52.92 \mathrm{~N}, 166.43 \mathrm{~W}$.

Depth:

Magnitude:

$70 \mathrm{~km}$

None computed.

Intensity IV: Unalaska (M).

22 January (G) Cook Inlet

Origin time: 020254.0

Epicenter: $\quad 60.24$ N., $152.33 \mathrm{~W}$.

Depth:

Magnitude:

$115 \mathrm{~km}$

None computed.

Intensity III: Anchorage (M).

27 January (G) Kenai Peninsula

origin time: $\quad 185259.2$

Epicenter: $\quad 60.37 \mathrm{~N} ., 151.12 \mathrm{~W}$.

Depth:

$70 \mathrm{~km}$

Magnitude: $\quad 4.7 \mathrm{mb}$

Intensity III: Anchorage (M), Eagle

River (M), Kenai (M), Soldotna (M).

28 January (G) Kenai Peninsula

origin time: 185306.8

Epicenter: $\quad 60.07 \mathrm{~N}, 151.33 \mathrm{~W}$.

Depth:

Magnitude: $\quad 4.5 \mathrm{mb}$

Intensity III: Kenai (M), Nikishka

(M).

Intensity II: Anchorage (M).

12 February (G) Cook Inlet

Origin time: $\quad 085638.9$

Epicenter: $\quad 59.45$ N., $152.62 \mathrm{~W}$.

Depth:

Magnitude:

$72 \mathrm{~km}$

$5.4 \mathrm{mb}$

Intensity IV: Anchor Point, Homer, Ouzinkie, Seldovia.

Intensity III: Kodiak, Kokhanok, Port Graham.

Intensity II: English Bay (M), Homer (M), King Salmon (M).

16 February (G) Southern Alaska

origin time: 205349.0

Epicenter: $\quad 61.31 \mathrm{~N} ., 144.89 \mathrm{~W}$.

Depth:

Normal.

Magnitude: $\quad 4.1 \mathrm{ML}(\mathrm{M})$

Intensity IV: Chitina.

6 March (G) Andreanof Islands, Aleutian Islands

Origin time:

Epicenter :

$\begin{array}{lll}18 & 40 & 23.6\end{array}$

Depth:

Magnitude:

$51.76 \mathrm{~N} ., 175.81 \mathrm{~W}$.

: $\quad 4.7 \mathrm{mb}$

Intensity II: Adak (M).
Alaska--Continued

20 March (G) Southern Alaska

origin time: 035905.0

Epicenter: $\quad 60.18 \mathrm{~N}, 153.61 \mathrm{~W}$.

Depth:

$153 \mathrm{~km}$

Magnitude: $\quad 4.9 \mathrm{mb}$

Intensity II: Anchorage (M), Homer

(M).

20 March (G) Southern Alaska

origin time: 081537.5

Epicenter: $\quad 59.84 \mathrm{~N} ., 153.24 \mathrm{~W}$.

Depth:

$134 \mathrm{~km}$

Magnitude:

$3.8 \mathrm{mb}$

Intensity III: Soldotna (M).

Intensity II: Homer (M), Kenai (M).

31 March (G) Southern Alaska

origin time: $\quad 0038 \quad 13.4$

Epicenter: $\quad 61.77 \mathrm{~N} ., 151.41 \mathrm{~W}$.

Depth:

Magnitude:

$90 \mathrm{~km}$

$5.1 \mathrm{mb}$

Intensity IV: Anchorage (M),

Chugiak, Cooper Landing, Girdwood, Kasilof, Kenai, Skwentna,

Talkeetna, Tyonek, Wasilla, Whittier, Willow.

Intensity III: Elmendorf AFB, Homer (M), Moose Pass, Palmer (M).

9 April (G) Kenai Peninsula

origin time: $\quad 171259.9$

Epicenter: $\quad 60.69 \mathrm{~N}, 151.84 \mathrm{~W}$.

Depth:

Magnitude: $\quad 4.5 \mathrm{mb}$

Intensity III: Anchorage (M), Kenai

(M).

Intensity II: Homer (M).

12 April (G) Kodiak Island region

origin time: 034203.5

Epicenter: $\quad 56.42 \mathrm{~N} ., 152.69 \mathrm{~W}$.

Depth:

$14 \mathrm{~km}$

Magnitude:

$6.0 \mathrm{mb}, 6.6 \mathrm{MS}, 6.5$ MS (B), 6.3 MS (P), $5.8 \mathrm{ML}(\mathrm{A})$

Intensity V: Sitkinak Island.

Intensity IV: Kodiak, old Harbor (M) .

Intensity III: Ouzinkie.

Intensity II: Olga Bay (M), Zechar Bay (M).

19 April (G) Southern Alaska

Origin time: $\quad 014903.5$

Epicenter: $\quad 60.14$ N. $153.54 \mathrm{~W}$.

Depth:

Intensity II: Anchorage (M).

19 April (G) Southern Alaska

origin time: $\quad 145218.1$

Fpicenter: $\quad 61.00 \mathrm{~N} ., 146.49 \mathrm{~W}$.

Depth:

Maqnitude: $\quad 3.3 \mathrm{ML}(\mathrm{M})$

$40 \mathrm{~km}$

Intensity IV: Valdez (M).

21 April (G) Central Alaska

origin time: $\quad 20 \quad 40 \quad 37.7$ 
Alaska--Continued

Epicenter :

Depth:

Magnitude:

$64.53 \mathrm{~N} ., 147.95 \mathrm{~W}$.

$30 \mathrm{~km}$

$3.7 \mathrm{ML}(\mathrm{M})$

Intensity II: College (M).

24 April (G) Andreanof Islands, Aleutian Islands

Origin time: $\quad 042847.0$

Epicenter: $\quad 51.64 \mathrm{~N}, 176.09 \mathrm{~W}$.

Depth :

Magnitude: $\quad 5.2 \mathrm{mb}, 4.8 \mathrm{MS}$

Intensity III: Adak.

5 May (G) Southern Alaska

Origin time: $\quad 053247.4$

Epicenter: $\quad 63.30 \mathrm{~N} ., 150.97 \mathrm{~W}$.

Depth:

$134 \mathrm{~km}$

Magnitude: $\quad 5.2 \mathrm{mb}$

Intensity IV: Skwentna, Talkeetna, Willow to wide Pass (M).

Intensity III: Anchorage to Palmer (M).

11 May (G) Andreanof Islands, Aleutian Islands

Origin time:

Epicenter:

$\begin{array}{llll}00 & 23 & 37.6\end{array}$

Depth:

Magnitude:

$51.67 \mathrm{~N} ., 176.10 \mathrm{~W}$.

$59 \mathrm{~km}$

$5.6 \mathrm{mb}, 5.9 \mathrm{MS}$, $5.8 \mathrm{MS}(\mathrm{B})$

Intensity IV: Adak (M).

12 May (G) Central Alaska

$\begin{array}{ll}\text { Origin time: } & 121603.9 \\ \text { Epicenter: } & 62.25 \mathrm{~N} ., 149.40 \mathrm{~W} . \\ \text { Depth: } & 67 \mathrm{~km} \\ \text { Magnitude: } & 5.1 \mathrm{mb}\end{array}$

Felt throughout the Susitna valley (press report).

Intensity IV: Anchorage, Chugiak, Elmendorf AFB, Gakona, Girdwood, Glennallen, Gold Creek (M), Homer, Kashwitna (M), McKinley Park', Palmer (M), Skwentna, Talkeetna, Wasilla (M), Whittier.

Intensity III: Fairbanks (M).

Intensity II: Ester, Moose Pass.

24 May (G) Andreanof Islands, Aleutian Islands

origin time:

Epicenter:

Depth:

Magnitude:

Intensity IV:

$06 \quad 16 \quad 55.4$

$51.23 \mathrm{~N} ., 179.21 \mathrm{~W}$.

$25 \mathrm{~km}$

$6.0 \mathrm{mb}, 6.7 \mathrm{MS}$, $6.4 \mathrm{mb}(\mathrm{P}), 6.2$ $\mathrm{MS}(\mathrm{P}), 6.4 \mathrm{MS}(\mathrm{B})$

Adak (M).

24 May (G) Andreanof Islands, Aleutian Islands

Origin time:

$\begin{array}{llll}09 & 53 & 03.4\end{array}$

Epicenter :

Depth:

$51.13 \mathrm{~N} ., 179.20 \mathrm{~W}$.

Magnitude:

Normal.

Intensity III: Adak.
Alaska--Continued

25 May (G) Central Alaska

origin time: $\quad 103957.4$

Epicenter: $\quad 64.55 \mathrm{~N} ., 152.59$ 认े.

Depth:

Magnitude:

Normal.

Intensity IV: Tanana.

31 May (G) Southern Alaska

Origin time: $\quad 182925.6$

Epicenter: $\quad 61.36 \mathrm{~N}, 149.70 \mathrm{~W}$.

Depth:

Magnitude :

$44 \mathrm{~km}$

Intensity II: $\operatorname{Palmer}(M)$.

10 June (G) Alaska Peninsula

Origin time: $\quad 082359.6$

Epicenter: $\quad 57.92 \mathrm{~N}, 156.72 \mathrm{~W}$.

Depth:

Magnitude:

$10 \mathrm{~km}$

$4.5 \mathrm{mb}, 3.9 \mathrm{MS}$, 4. $6 \mathrm{ML}(\mathrm{M})$

Intensity II: Egegik (M).

Intensity I: King Salmon (M).

10 June (G) Southern Alaska

Origin time: $\quad 193510.2$

Epicenter: $\quad 60.30 \mathrm{~N} ., 146.45 \mathrm{~W}$.

Depth:

Magnitude:

$20 \mathrm{~km}$

Intensity IV:

$4.8 \mathrm{mb}, 4.7 \mathrm{ML}(\mathrm{M})$

Intensity III: Anchorage (M), Valdez (M).

12 June (G) Kenai Peninsula, Alaska

Origin time: 073039.3

Epicenter: $\quad 59.86 \mathrm{~N}, 150.76 \mathrm{~W}$.

Depth:

Maqnitude:

$55 \mathrm{~km}$

Intensity III: Homer (M).

22 June (G) Andreanof Islands, Aleutian Islands

origin time: $\quad \begin{array}{llll}05 & 41 & 27.7\end{array}$

Epicenter: $\quad 51.61 \mathrm{~N} ., 179.41 \mathrm{~W}$.

Depth:

Normal.

Magnitude: $\quad 4.8 \mathrm{mb}, 5.9 \mathrm{MS}$,

Intensity III: $\begin{gathered}5.0 \mathrm{ML}(\mathrm{M}) \\ \text { Adak (M) }\end{gathered}$

13 July (G) Southern Alaska

Origin time: $\quad 152733.5$

Epicenter: $\quad 62.11 \mathrm{~N} ., 149.95 \mathrm{~W}$.

Depth:

Magnitude: $\quad 3.5 \mathrm{ML}(\mathrm{M})$

Intensity II: $\quad \operatorname{Palmer}(M)$.

16 July (G) Central Alaska

Origin time: 050302.3

Epicenter: $\quad 63.57 \mathrm{~N}, 150.52 \mathrm{~W}$.

Depth:

$31 \mathrm{~km}$

Magnitude: $\quad 3.5 \mathrm{ML}(\mathrm{M})$

Intensity III: McKinley Park (M).

19 July (A) Southern Alaska

Origin time: $\quad 185432.8$

Epicenter: $\quad 61.33 \mathrm{~N}, 149.98 \mathrm{~W}$.

Depth:

$13 \mathrm{~km}$

Magnitude: $\quad 3.0 \mathrm{ML}(\mathrm{M})$ 
Intensity II: Chugiak (M), Eagle River (M), Susitna Flats--15 km north of Anchorage (A).

$23 \mathrm{July}$ (G) Central Alaska

origin time: $\quad 151935.5$

Epicenter: $\quad 63.31 \mathrm{~N} ., 147.26 \mathrm{~W}$.

Depth:

Magnitude: $\quad 5.0 \mathrm{mb}, 4.8 \mathrm{ML}(\mathrm{M})$

Intensity III: Throughout central Alaska (M).

27 July (G) Central Alaska

Origin time: $\quad 141848.0$

Epicenter: $\quad 65.00 \mathrm{~N} ., 147.60 \mathrm{~W}$.

Depth:

Magnitude:

$20 \mathrm{~km}$

$3.8 \mathrm{ML}(\mathrm{A})$

Intensity IV: Fairbanks.

27 July (G) Central Alaska

origin time: 155142.2

Epicenter:

Depth:

$64.85 \mathrm{~N}, 147.59 \mathrm{~W}$.

Magnitude:

$10 \mathrm{~km}$

Intensity III: Fairbanks (press report).

27 July (G) Central Alaska

Origin time: $\quad 171121.1$

Epicenter: $\quad 64.93 \mathrm{~N} ., 148.02 \mathrm{~W}$.

Depth:

$10 \mathrm{~km}$

Magnitude: $\quad 3.7 \mathrm{ML}(\mathrm{A})$

Intensity III: Fairbanks (press report).

3 August (G) Kenai Peninsula

origin time: 063330.9

Fpicenter: $\quad 59.78 \mathrm{~N}, 151.15 \mathrm{~W}$.

Depth:

Magnitude:

$89 \mathrm{~km}$

None computed.

Intensity III: Homer (M).

8 August (G) Southern Alaska

origin time: 093003.3

Epicenter: $\quad 61.39$ N. $146.91 \mathrm{~W}$.

Depth:

Magnitude:

$53 \mathrm{~km}$

Intensity $V$ : Palmer (few windows

cracked).

Intensity IV: Delta Junction,

Glennallen, Valdez, Whittier.

Intensity III: Anchorage (M), Girdwood.

Intensity II: Chugiak.

13 August (G) Southern Alaska

origin time: 004941.0

Epicenter: $\quad 62.28 \mathrm{~N} ., 149.71 \mathrm{~W}$.

Depth:

Magnitude:

$65 \mathrm{~km}$

Intensity IV: Anchorage, Chugiak,

Skwentna.

Intensity III: Big Lake (M), Palmer

(M), Wasilla (M).

18 August (G) Southern Alaska

Origin time: $\quad 185228.4$

Epicenter
Depth:

Magnitude:

$123 \mathrm{~km}$

Intensity VI: Clam Gulch (cracked

$5.4 \mathrm{mb}, 5.7 \mathrm{mb}(\mathrm{B})$ plasterboard, hairline cracks in exterior cinderblock walls, small objects shifted).

Intensity V: Sterling.

Intensity IV: Anchorage, Chugiak, Cooper Landing, Eagle River, Girdwood, Homer, Kenai, Larsen Bay, Moose Pass, Port Lions, Seldovia, Skwentna, Tyonek, Whittier.

Intensity III: Cordova (M), Healey (M), King Salmon (M), Kodiak (M), Soldotna, Spenard, Willow.

Intensity II: Fairbanks (M), Nikishka.

22 August (G) Central Alaska

origin time: $\quad 041355.3$

Epicenter: $\quad 65.16 \mathrm{~N} ., 151.99 \mathrm{~W}$.

Depth:

Magnitude:

$14 \mathrm{~km}$

Intensity II: Tanana (M).

22 August (G) Central Alaska

origin time: 095324.2

Epicenter: $\quad 65.23 \mathrm{~N}, 152.12 \mathrm{~W}$.

Depth:

Magnitude:

$17 \mathrm{~km}$

Intensity II: Tanana (M).

22 August (A) Central Alaska

origin time: $\quad 101202.8$

Epicenter: $\quad 64.92 \mathrm{~N}, 152.53 \mathrm{~W}$.

Depth:

Magnitude: $\quad 3.8 \mathrm{ML}(\mathrm{A})$

Intensity II: Tanana.

22 August (A) Central Alaska

Origin time: $\quad 102908.0$

Epicenter: $\quad 64.99 \mathrm{~N}, 152.31 \mathrm{~W}$.

Depth:

Magnitude:

$1 \mathrm{~km}$

$3.4 \mathrm{ML}(\mathrm{A})$

Intensity II: Tanana.

26 Auqust (G) Central Alaska

Origin time: $\quad 134431.2$

Epicenter: $\quad 65.08 \mathrm{~N} ., 152.36 \mathrm{~W}$.

Depth:

Magnitude: Normal.

Intensity II: Tanana (M).

3 September (A) Central Alaska

origin time: 062705.4

Epicenter: $\quad 64.58 \mathrm{~N} ., 147.16 \mathrm{~W}$.

Depth:

Magnitude: $\quad 3.9 \mathrm{ML}$

Intensity II: Eielson AFB (A), Fairbanks (A).

18 September (G) Central Alaska

Origin time: $\quad 170254.9$

Epicenter: $\quad 63.66 \mathrm{~N}, 147.59 \mathrm{~W}$.

Depth:

Magnitude:

$88 \mathrm{~km}$

Cantwell. Us

Cantwell, Usibelli. 
Alaska--Continued

Intensity II: Talkeetna (A), Willow (A).

19 September (G) Southern Alaska

origin time: 083756.0

Epicenter: $\quad 61.34 \mathrm{~N}, 147.18 \mathrm{~W}$.

Depth:

Magnitude: $\quad 3.9 \mathrm{ML}(\mathrm{M})$

Intensity III: Anchorage to Valdez (A).

Intensity II: Gakona, Valdez.

20 September (G) Southern Alaska

origin time: $\quad 114605.9$

Epicenter: $\quad 61.92 \mathrm{~N} ., 149.23 \mathrm{~W}$.

Depth: $8 \mathrm{~km}$

Magnitude: $\quad 3.8 \mathrm{ML}(\mathrm{M})$

Intensity IV: Girdwood, Palmer,

Sutton.

Incensity II: Anchorage (M), Independence Mine area (M), Skwentna.

21 September (G) Southern Alaska

origin time: $\quad 144519.6$

Epicenter: $\quad 61.11 \mathrm{~N} ., 151.81 \mathrm{~W}$.

Depth:

Magnitude :

$81 \mathrm{~km}$

$4.5 \mathrm{mb}$

Intensity IV: Anchorage (M), Eagle

River (M), Girdwood, Kenai, Palmer, Soldotna, sterling.

Intensity II: Sutton.

25 September (G) Andreanof Islands,

Aleutian Islands

origin time: 093701.9

Epicenter: $\quad 51.79 \mathrm{~N}, 175.28 \mathrm{~W}$.

Depth:

Magnitude: $\quad 4.6 \mathrm{mb}$

Intensity II: Adak (telephone

report).

26 September (G) Central Alaska

Origin time: $\quad 160818.6$

Epicenter: $\quad 64.99 \mathrm{~N} ., 147.55 \mathrm{~W}$.

Depth:

Magnitude: $\quad 3.7 \mathrm{mb}, 3.9 \mathrm{ML}(\mathrm{M})$

Intensity III: Eielson AFB (A),

Fairbanks, North Pole (A).

28 September (G) Central Alaska

Origin time: $2353 \quad 13.7$

Epicenter: $\quad 63.99 \mathrm{~N} .147 .71 \mathrm{~W}$.

Depth:

Magnitude: $\quad 4.4 \mathrm{mb}, 4.5 \mathrm{ML}(\mathrm{M})$

Intensity III: Big Delta (M), Delta

Junction $(A)$, Fairbanks $(M)$, Healey

(M), Nenana (M), North Pole (M).

Solcha (A).

4 October (G) Andreanof Islands, Aleutian Islands

origin time: $\quad 185300.1$

Epicenter: $\quad 51.81 \mathrm{~N}, 177.05 \mathrm{~W}$.

Depth :

Magnitude:

$58 \mathrm{~km}$

Intensity IV: Adak Island (M).

\section{Alaska--Continued}

4 October (G) Near Islands, Aleutian

Islands

Origin time: $\quad 195517.5$

Epicenter: $\quad 50.93$ N., 173.53 E.

Depth:

Magnitude: $\quad 5.3 \mathrm{mb}, 5.0 \mathrm{MS}$

Intensity III: Shemya Island (M).

6 october (G) Southern Alaska

Origin time: 055405.2

Epicenter: $\quad 61.93 \mathrm{~N}, 150.67 \mathrm{~W}$.

Depth:

Magnitude: $\quad 4.6 \mathrm{ML}(\mathrm{M})$

Intensity III: Willow-Hatcher Pass area (M).

Intensity II: Palmer (M), Wasilla (M).

17 October (G) Andreanof Islands, Aleutian origin time: 205048.7

Epicenter: $\quad 51.72 \mathrm{~N}, 176.94 \mathrm{~W}$.

Depth :

Magnitude: $\quad 5.0 \mathrm{mb}$

Eighty-six questionnaires were completed for this earthquake from the Naval Air Station, Adak Island. The evaluated intensities from the information on these questionnaires ranged from intensity IV to VI.

27 October (G) Southern Alaska

origin time: $\quad 042931.5$

Epicenter: $\quad 62.20 \mathrm{~N}, 151.05 \mathrm{~W}$.

Depth:

Magnitude: None computed.

Intensity II: Talkeetna.

30 October (G) Southern Alaska

origin time: $\quad 111138.4$

Epicenter: $\quad 60.96 \mathrm{~N}, 150.32 \mathrm{~W}$.

Depth :

Magnitude: $\quad 3.3 \mathrm{mb}$

Intensity III: Anchorage area (M).

31 October (G) Southern Alaska

Origin time: $\quad 122830.1$

Epicenter: $\quad 61.91 \mathrm{~N} ., 149.57 \mathrm{~W}$.

Depth:

Magnitude: $\quad 3.5 \mathrm{mb}, 3.4 \mathrm{ML}(\mathrm{M})$

Intensity II: Palmer area (M).

14 November (G) Central Alaska

origin time: 222745.7

Epicenter: $\quad 64.54 \mathrm{~N}, 147.03 \mathrm{~W}$.

Depth:

Magnitude:

$25 \mathrm{~km}$

Intensity II: Fairbanks area (M).

19 November (G) Near Islanis, Aleutian Islands

Origin time: $\quad 194235.7$

Epicenter: $\quad 52.70 \mathrm{~N}, 172.48 \mathrm{~F}$.

Depth:

Magnitude:

$47 \mathrm{~km}$

$5.3 \mathrm{mb}(\mathrm{G}), 5.2 \mathrm{MS}(\mathrm{G})$

Intensity $V$ : Shemya Island. 
Alaska--Continued

24 November (G) Southern Alaska

origin time: 002812.8

Epicenter: $\quad 62.03 \mathrm{~N} ., 150.52 \mathrm{~W}$.

Depth:

Intensity II: Palmer (M), Talkeetna (M).

24 November (G) Southern Alaska

origin time: 085045.4

Epicenter: $\quad 61.99 \mathrm{~N}, 150.51 \mathrm{~W}$.

Depth:

Intensity II: Willow (M).

2 December (G) Kenai Peninsula

origin time: $\quad 215720.0$

Epicenter: $\quad 59.69$ N., $151.66 \mathrm{~W}$.

Depth: $\quad 13 \mathrm{~km}$

Magnitude: $\quad 3.7 \mathrm{ML}(\mathrm{M})$

Intensity $\mathrm{V}$ : Homer.

3 December (G) Central Alaska

origin time: $\quad 193931.2$

Epicenter: $\quad 62.31 \mathrm{~N} .149 .75 \mathrm{~W}$.

Depth :

Magnitude:

Intensity IV: Chugiak, Talkeetna

(M).

Intensity III: Anchorage (M), Palmer (M), Willow (M).

Intensity II: Wasilla.

4 December (G) Central Alaska

origin time: $\quad 121106.4$

Epicenter: $\quad 65.04 \mathrm{~N} ., 147.51 \mathrm{~W}$.

Depth: $\quad 24 \mathrm{~km}$

Magnitude: $\quad 3.3 \mathrm{ML}$ (M)

Intensity II: Fairbanks (M).

8 December (G) Northern Alaska

origin time: $\quad 100151.5$

Epicenter: $\quad 68.33 \mathrm{~N} ., 145.17 \mathrm{~W}$.

Depth:

Normal.

Magnitude:

4.0 ML(M)

Intensity II: Fairbanks (M).

15 December (G) Rat Islands, Aleutian Islands

Origin time: $\quad 083034.7$

Epicenter: $\quad 52.11 \mathrm{~N} ., 175.23 \mathrm{E}$.

Depth:

Magnitude :

$47 \mathrm{~km}$

$5.6 \mathrm{mb}, 5.6 \mathrm{MS}$, $5.4 \operatorname{MS}(B)$

Intensity $V$ : Shemya AFB (light

furniture and small objects shifted).

Intensity IV: Attu.

17 December (G) Central Alaska

origin time: 131526.0

Epicenter :

Depth:

$63.95 \mathrm{~N} ., 147.42 \mathrm{~W}$.

Magnitude: $\quad 4.8 \mathrm{mb}, 4.6 \mathrm{ML}(\mathrm{M})$

Intensity IV: Clear AFB (M), Ester,
Alaska--Continued

22 December (G) Alaska Peninsula

origin time: 032529.9

Epicenter: $\quad 55.57 \mathrm{~N}, 160.37 \mathrm{~W}$.

Depth:

Magnitude:

$12 \mathrm{~km}$

Intensity IV: Sand Point (M).

24 December (G) Céntral Alaska

origin time: $\quad 131308.1$

Epicenter: $\quad 63.56 \mathrm{~N} ., 157.59 \mathrm{~W}$.

Depth:

Magnitude: $\quad 5.0 \mathrm{mb}(\mathrm{G}), 4.4 \mathrm{MS}(\mathrm{G})$, $5.3 \mathrm{ML}(\mathrm{M})$

Intensity IV: Galena Airport (people were awakened and the control tower was evacuated).

Arizona

10 March (P) Baja California

Origin time: $\quad 203436.2$

Epicenter: $\quad 32.33 \mathrm{~N}, 115.03 \mathrm{~W}$.

Depth: $\quad 5 \mathrm{~km}$

Maqnitude: $\quad 3.5 \mathrm{ML}$

Intensity III: Yuma (telephone

report).

Il March (G) Baja California

origin time: $\quad 235746.8$

Epicenter: $\quad 32.26 \mathrm{~N}, 115.12 \mathrm{~W}$.

Depth:

Magnitude: $\quad 4.8 \mathrm{mb}, 5.0 \mathrm{ML}(\mathrm{P})$

This earthquake destroyed approximately 30 houses, cracked dozens of buildings, and interrupted electric power and telephone communications in the cities of San Luis, Sonora, and Luis Sanchez, Mexico.

Intensity VI: Arizona--Yuma (cracked plaster).

Intensity III: California--El Centro (press report).

12 March (G) Baja California

origin time: $\quad 003015.7$

Epicenter: $\quad 32.23 \mathrm{~N} ., 115.14 \mathrm{~W}$.

Depth: $\quad 5 \mathrm{~km}$

Magnitude: $\quad 4.5 \mathrm{mb}, 4.7 \mathrm{ML}(\mathrm{P})$

Intensity IV: Arizona--Yuma.

Intensity III: California--southern Imperial Valley (telephone report).

12 March (G) Baja California

Origin time: $\quad \begin{array}{lll}18 & 42 & 24.3\end{array}$

Epicenter: $\quad 32.26 \mathrm{~N} ., 115.11 \mathrm{~W}$.

Depth: $\quad 5 \mathrm{~km}$

Magnitude: $\quad 4.9 \mathrm{mb}, 4.9 \mathrm{ML}(\mathrm{P})$

Intensity VI:

Arizona-Yuma (cracked plaster, cracked streets).

Intensity IV:

Arizona--Somerton.

California--Imperial, Winterhaven. 
16 March (P) Baja California

origin time: 015110.1

Epicenter: $\quad 32.30 \mathrm{~N}, 115.12 \mathrm{~W}$.

Depth :

Magnitude:

$5 \mathrm{~km}$

Intensity IV: Yuma.

5 May (P) Baja California

origin time: 210315.8

See California listing.

\section{Arkansas}

31 August (S) New Madrid, Missouri region origin time: 003100.3

See Tennessee listing.

23 September (S) Southern Arkansas

origin time: 073357.5

Epicenter: $\quad 33.65 \mathrm{~N} ., 91.89 \mathrm{~W}$.

Depth:

$2 \mathrm{~km}$

Magnitude:

$3.1 \mathrm{mbLg}$

Intensity IV: Wilmar.

21 November (S) Northeastern Arkansas

Origin time: 233122.1

Epicenter: $\quad 35.97 \mathrm{~N}, 89.92 \mathrm{~W}$.

Depth:

$10 \mathrm{~km}$

Magnitude:

$2.4 \mathrm{MI}$

Intensity II: Blytheville.

\section{California}

4 January (B) Northern California

Origin time: $\quad 122300.5$

Epicenter: $\quad 40.65 \mathrm{~N} ., 124.77 \mathrm{~W}$.

Depth :

$27 \mathrm{~km}$

Magnitude: $\quad 3.7 \mathrm{ML}$

Intensity IV: Ferndale, Fortuna.

Intensity III: Eel River Valley.

6 January (B) Northern California

origin time: $\quad 104244.8$

Epicenter: $\quad 39.42 \mathrm{~N}, 123.33 \mathrm{~W}$.

Depth:

$14 \mathrm{~km}$

Magnitude: $\quad 2.9 \mathrm{ML}$

Intensity IV: Willits.

14 January (B) Northern California

origin time: $\quad 154545.6$

Epicenter: $\quad 38.81 \mathrm{~N}, 122.79 \mathrm{~W}$.

Depth: $\quad 5 \mathrm{~km}$

Magnitude: $\quad 3.1 \mathrm{ML}$

Intensity III: Southern Lake County (press report).

15 January (B) Northern California

origin time: $\quad 143826.2$

Epicenter: $\quad 39.14 \mathrm{~N} ., 123.25 \mathrm{~W}$.

Depth :

Magnitude:

$5 \mathrm{~km}$

Intensity IV: Ukiah (press report).
Intensity III: Willits (press report).

17 January ( $B$ ) Northern California

origin time: $\quad 205912.9$

Epicenter: $\quad 39.15 \mathrm{~N} ., 123.26 \mathrm{~W}$.

Depth:

Magnitude:

$8 \mathrm{~km}$

$2.9 \mathrm{ML}$

Intensity $V$ : Ukiah (hairline crack in the wall, dishes rattled, buildings swayed--press report).

25 January (P) Southern California

origin time: $\quad 104050.0$

Epicenter: $\quad 34.32 \mathrm{~N} ., 118.33 \mathrm{~W}$.

Depth: $\quad 5 \mathrm{~km}$

Magnitude: $\quad 2.7 \mathrm{ML}$

Intensity III: La Crescenta, sylmar.

28 January (B) Northern California

Origin time: 084902.5

Epicenter: $\quad 39.40 \mathrm{~N} ., 123.35 \mathrm{~W}$.

Depth:

Magnitude: $\quad 2.5 \mathrm{ML}(\mathrm{B})$

Intensity $V:$ willits (furniture and small objects moved; people awakened; windows, doors, dishes rattled).

31 January (P) Southern California

origin time: $\quad 141810.0$

Epicenter: $\quad 34.18 \mathrm{~N}, 118.63 \mathrm{~W}$.

Depth:

Magnitude: $\quad 2.1 \mathrm{MI}$

Intensity III: Canoga Park, Chatsworth.

6 February (P) Southern California

origin time: 003925.8

Epicenter: $\quad 34.03 \mathrm{~N}, 116.78 \mathrm{~W}$.

Depth :

Magnitude: $\quad 3.1 \mathrm{M}$

Intensity III: Palm Springs.

6 February (P) Southern California

origin time: 010128.9

Epicenter: $\quad 34.03 \mathrm{~N}, 116.78 \mathrm{~W}$.

Depth: $\quad 6 \mathrm{~km}$

Magnitude: $\quad 3.3 \mathrm{ML}$

Intensity III: Palm Springs.

6 February (P) Imperial Valley

origin time: $\quad 125714.2$

Epicenter: $\quad 33.25 \mathrm{~N} ., 115.57 \mathrm{~W}$.

Depth: $\quad 4 \mathrm{~km}$

Magnitude: $\quad 3.4 \mathrm{ML}$

Intensity IV: Niland (many awakened; windows, doors, dishes rattled; buildings shook).

Intensity III: Brawley (telephone report).

7 February (B) Northern California

Origin time: $\quad 133922.7$

Epicenter: $\quad 39.41 \mathrm{~N}, 123.17 \mathrm{~W}$.

Depth:

$5 \mathrm{~km}$

Magnitude: $\quad 2.7 \mathrm{ML}(\mathrm{B})$

Intensity IV: Willits (many awak- 
ened; small objects shifted; buildings shook; windows, doors, dishes rattled).

11 February (P) Imperial Valley

Origin time: 014530.5

Epicenter: $\quad 33.02 \mathrm{~N}, 115.53 \mathrm{~W}$.

Depth:

$7 \mathrm{~km} \mathrm{~N}, 115.53 \mathrm{~W}$.

Magnitude: $\quad 2.5 \mathrm{ML}$

Intensity II: Brawley.

11 February (P) Imperial Valley

oriqin time: 014551.0

Epicenter: $\quad 33.02 \mathrm{~N}, 115.53 \mathrm{~W}$.

Depth :

$5 \mathrm{~km}$

Magnitude: $\quad 2.5 \mathrm{ML}$

Intensity II: Brawley.

11 February (P) Imperial Valley,

Origin time: 022551.4

Epicenter: $\quad 33.02 \mathrm{~N}, 115.53 \mathrm{~W}$.

Magnitude: $\quad 3.1 \mathrm{ML}$

Depth: $6 \mathrm{~km}$

Intensity III: Brawley.

13 February (P) Southern California

origin time: $\quad 180406.3$

Epicenter: $\quad 34.02$ N., $117.22 \mathrm{~W}$.

Depth:

Magnitude:

$6 \mathrm{~km}$

$2.9 \mathrm{MI}$

Intensity II: Redlands.

14 February (B) Northern California

Origin time: $\quad 163346.9$

Epicenter: $\quad 38.41 \mathrm{~N}, 122.65 \mathrm{~W}$.

Depth:

Magnitude:

$8 \mathrm{~km}$

Intensity II: Santa Rosa.

14 February (B) Northern California

Origin time: 200309.5

Epicenter: $\quad 38.42 \mathrm{~N} ., 122.66 \mathrm{~W}$.

Depth:

$9 \mathrm{~km}$

Magnitude:

$2.5 \mathrm{ML}$

Intensity II: Santa Rosa.

14 February (B) Northern California

origin time: $\quad 210355.4$

Epicenter: $\quad 40.30 \mathrm{~N} ., 124.27 \mathrm{~W}$.

Depth:

Magnitude: $\quad 4.0 \mathrm{MI}$

Intensity IV: Rio Dell.

Intensity III: Ferndale, Fortuna.

23 February (P) Imperial Valley

origin time: $\quad 164303.7$

Epicenter:

Depth :

Magnitude :

$32.82 \mathrm{~N} ., 115.60 \mathrm{~W}$.

$19 \mathrm{~km}$

Intensity IV: Imperial, Seeley.

1 March (P) Southern California

Origin time: $\quad 045431.2$

Epicenter: $\quad 34.53 \mathrm{~N} ., 116.77 \mathrm{~W}$.

Depth:

Magnitude: $\quad 4.4 \mathrm{ML}, 4.4 \mathrm{ML}(\mathrm{B})$

Intensity VI: White Water (cracked plaster; light furniture shifted).
Intensity V: Colton, Fawnskin, Highland.

Intensity IV: Angelus Oaks, Apple Valley, Big Bear City, Bryn Mawr, Chino, Crestline, Etiwanda, Green Valley Lake, Montrose, Mount Baldy, Palomar Mountain, Rimforest, San Bernardino, Twin Peaks.

Intensity III: Barstow, Big Bear Lake, Crest Park, Del Rosa, Isake Arrowhead, Lucerne Valley, Newberry Springs, Norton AFB, Victorville (press report).

Intensity II: Azusa, Blue Jay, Burbank, Daggett, West Covina (press report), Wrightwood.

8 March (P) Southern California

origin time: $\quad 144934.9$

Epicenter: $\quad 33.83$ N., 117.88 W.

Depth:

Magnitude:

$5 \mathrm{~km}$

$2.9 \mathrm{ML}$

Intensity IV: Anaheim (Brookhurst Center, Sunkist), Fullerton, Long Beach, Orange, Placentia, Santa Ana, Westminster.

Intensity III: Brea (press report), Buena Park (press report), Cypress (press report), Garden Grove, La Mirada, Midway City, Stanton, Sunny Hills, Tustin (press report), Yorba Linda.

11 March (G) Baja California

origin time: $\quad 235746.8$

See Arizona listing.

12 March (G) Baja California origin time: 003015.7

See Arizona listing.

12 March (G) Baja California

origin time: $\quad 184224.3$

See Arizona listing.

13 March (P) Southern California

Origin time: $\quad \begin{array}{llll}16 & 38 & 15.7\end{array}$

Epicenter: $\quad 33.93 \mathrm{~N}, 117.98 \mathrm{~W}$.

Depth :

Magnitude:

$4 \mathrm{~km}$

$3.2 \mathrm{ML}$

Intensity VI: La Mirada (cracked plaster).

Intensity V: Buena Park, La Habra, Surfside.

Intensity IV: Anaheim (press report), Bellflower, Fullerton, Perry, Pico Rivera, Whittier.

Intensity III: Alhambra, Brea (press report), Compton, Manhattan Beach, Rowl and Heights.

Intensity II: La Puente, Norwalk, Santa Ana.

14 March (P) Southern California Origin time: $\quad 160951.2$ 


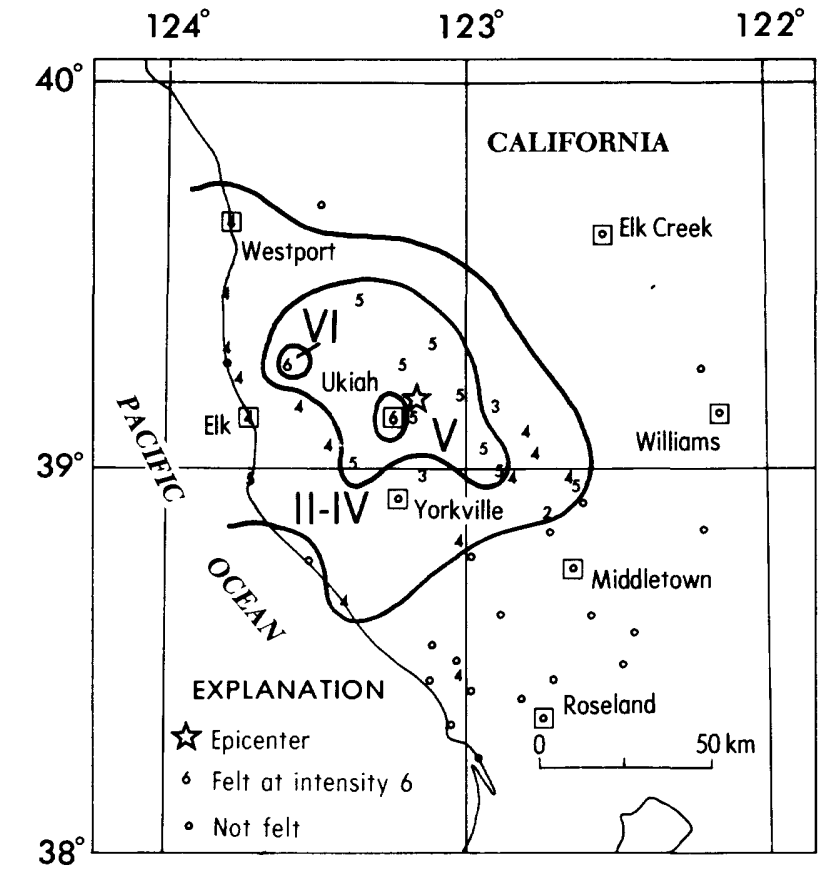

Epicenter:

Depth:

Magnitude:

Intensity IV:

-33.95 N., $117.97 \mathrm{~W}$.

$4 \mathrm{~km}$

$2.5 \mathrm{ML}$

Intensity III: Fullerton, Santa $\mathrm{Fe}$ Springs, Whittier.

15 March (P) Imperial Valley

Origin time:

$0402 \quad 49.4$

Epicenter:

$32.78 \mathrm{~N} ., 115.53 \mathrm{~W}$.

Depth :

$5 \mathrm{~km}$

Magnitude:

$2.9 \mathrm{ML}$

Intensity III: El Centro, Imperial (telephone report).

15 March (P) Imperial Valley

origin time: $\quad 040939.7$

Epicenter: $\quad 32.78$ N., $115.57 \mathrm{~W}$.

Depth:

Magnitude:

$5 \mathrm{~km}$

$3.2 \mathrm{ML}$

Intensity III: El Centro, Imperial (telephone report).

20 March (P) Southern California

origin time: $\quad 000756.7$

Epicenter: $\quad 36.00 \mathrm{~N}, 117.87 \mathrm{~W}$.

Depth :

Magnitude: $\quad 3.0 \mathrm{ML}, 3.6 \mathrm{ML}(\mathrm{B})$

Intensity III: Haiwee Reservoir

(telephone report).

21 March (B) Northern California

Origin time: $\quad 140512.1$

Epicenter: $\quad 40.44 \mathrm{~N}, 124.45 \mathrm{~W}$.

Depth :

Magnitude:

$11 \mathrm{~km}$

$3.5 \mathrm{ML}$

Intensity IV: Bayside, Loleta, Rio

Dell, Scotia.

Intensity III: Ferndale.

26 March (B) Northern California

Origin time: $\quad 002704.8$

Epicenter: $\quad 39.21 \mathrm{~N} ., 123.17 \mathrm{~W}$.

Depth :

$9 \mathrm{~km}$

Magnitude:

$4.9 \mathrm{mb}(\mathrm{G}), 4.4 \mathrm{ML}$

This earthquake was felt over an area of about $8,200 \mathrm{sq} \mathrm{km}$ centered in the Ukiah area (fig. 7).

Loss of about $\$ 10,000$ from glass bottles and containers being thrown to the floor and broken was sustained by grocery and 1 iquor stores in the Ukiah area. This earthquake triggered three accelerographs located at Coyote Dam near IJkiah (Porcella, 1978a). The accelerographs are located at the center crest, center toe, and south abutment of the dam. Maximum acceleration recorded at the crest was $0.30 \mathrm{~g}$, at the toe was $0.34 \mathrm{~g}$, and at the south abutment was $0.20 \mathrm{~g}$.

The press reported damage at a new warehouse at the Parducci Winery south of Ukiah. Huge storage tanks in the warehouse, welded to plates
FIGURE 7.--Isoseismal map for the northern California earthquake of 26 March 1978, 002704.4 UTC. Roman numerals represent Modified Mercalli intensities between isoseismals; Arabic numerals are used to represent these intensities at specific sitea. on the floor to hold them rigid, contained 200 tons of liquid. The liquid moved and about $1,000 \mathrm{gal}-$ lons spilled, damaging the tanks. Liquid in free-floating tanks in another building sustained minor spillage, but there was no damage to the tanks. The drop ceiling in the new warehouse was also damaged.

Intensity VI: Comptche (windows broken), Ukiah (pictures fell, glass bottles and jars broken in grocery and 1 iquor stores, burglar alarm set off, damage to Parducci Winery).

Intensity V: Boonville, Clearlake Highlands, Finley, Kelseyville, Lake port, Manchester, Potter Valley, Redwood Valley, Talmage, Willits, witter springs.

Intensity IV: Albion, Clearlake Park, Cloverdale, Elk, Fort Bragg, Glenhaven, Lucerne, Mendocino, Monte Rio, Navarro, Philo, Stewarts Point, Westport.

Intensity III: Hopland, Upper Lake. Intensity II: $\quad$ Loch Lomond. 
26 March (B) Northern California

origin time: $\quad 003411.9$

Epicenter: $\quad 39.21 \mathrm{~N}, 123.14 \mathrm{~W}$.

Depth:

Magnitude:

$5 \mathrm{~km}$

Intensity IV: Talmage, Ukiah.

Intensity III: Willits.

26 March (B) Northern California

Origin time: 011910.0

Epicenter: $\quad 39.20 \mathrm{~N}, 123.17 \mathrm{~W}$.

Depth:

Magnitude: $\quad 3.6 \mathrm{mb}(\mathrm{G}), 3.6 \mathrm{ML}$

Intensity IV: Talmage, Ukiah.

Intensity III: Willits.

26 March (B) Northern California

Origin time: 022916.9

Epicenter: $\quad 39.21 \mathrm{~N}, 123.17 \mathrm{~W}$.

Depth: $\quad 5 \mathrm{~km}$

Magnitude: $\quad 3.3 \mathrm{ML}$

Intensity IV: Talmage, Ukiah.

Intensity III: Willits.

26 March (B) Northern California

Origin time: $\quad 042818.6$

Epicenter: $\quad 39.23 \mathrm{~N}, 123.19 \mathrm{~W}$.

Depth:

$3 \mathrm{~km}$

Intensity IV: Talmage, Ukiah.

Intensity III: Comptche, Willits.

27 March (B) Northern California

Origin time: 084403.0

Fpicenter: $\quad 40.43 \mathrm{~N}, 124.31 \mathrm{~W}$.

Depth:

Magni tude:

$14 \mathrm{~km}$

$3.2 \mathrm{ML}$

Intensity IV: Ferndale, Fortuna, Rio Dell.

Intensity III: Scotia (press

report), Weotl.

1 April (P) Southern California

Origin time: $\quad 105227.4$

Epicenter: $\quad 34.20 \mathrm{~N} ., 116.97 \mathrm{~W}$.

Depth :

$6 \mathrm{~km}$

Magnitude: $\quad 4.2 \mathrm{ML}, 3.8 \mathrm{mb}(\mathrm{G})$

Intensity $V:$ Angelus Oaks, Big Bear City, Llano, Rosamund.

Intensity IV: Big Bear Lake, Blue Jay, Bryn Mawr, Cedar Glen, Coachella, Fawn-skin, Forest Falls, Green Valley Lake, Highland, Loma Linda, Lucerne Valley, Redlands, Running Springs, Sunnymead, Yucaipa.

Intensity III: Palm springs.

4 April (B) Northern California

oriqin time: 032331.8

Epicenter: $\quad 37.84 \mathrm{~N} ., 122.06 \mathrm{~W}$.

Depth:

Magnitude:

$8 \mathrm{~km}$.

$2.5 \mathrm{ML}$

Intensity II: Alamo (R), Walnut

Creek (B).

8 April (B) Northern California origin time:
Epicenter:

Depth:

Magnitude:

Intensity IV: Talmage, Willits.

Intensity III : Redwood Valley, Ukiah.

14 April (P) Central California

origin time: 100105.2

Epicenter: $\quad 35.28 \mathrm{~N}, 116.83 \mathrm{~W}$.

Depth:

Magnitude:

$5 \mathrm{~km}$

Intensity II: Goldestone Tracking

Station.

18 April (P) Southern California

Origin time: $\quad 224236.2$

Epicenter: $\quad 33.88 \mathrm{~N}, 117.55 \mathrm{~W}$.

Depth:

$6 \mathrm{~km}$

Magnitude:

3. $0 \mathrm{ML}$

Intensity II: Corona, Riverside.

22 April (P) Southern California

origin time: 130427.8

Epicenter: $\quad 34.05 \mathrm{~N},, 118.97 \mathrm{~W}$.

Depth:

Magnitude:

$14 \mathrm{~km}$

Intensity III: Malibu to point Dume (P).

25 April (P) Imperial Valley region

Origin time: 045838.2

Epicenter: $\quad 33.00 \mathrm{~N}, 115.58 \mathrm{~W}$.

Depth:

Magnitude:

$11 \mathrm{~km}$

Intensity II: Brawley (P).

25 April (P) Imperial Valley region

Origin time: $\quad 051325.3$

Epicenter: $\quad 33.00 \mathrm{~N}, 115.58 \mathrm{~W}$.

Depth:

Magnitude: $\quad 2.3 \mathrm{ML}$

Intensity II: Brawley (P).

25 April (P) Southern California

Origin time: $\quad 215630.2$

Epicenter: $\quad 33.72 \mathrm{~N}, 118.37 \mathrm{~W}$.

Depth:

Magnitude:

$4 \mathrm{~km}$

Intensity II: Long Beach (P).

26 April (P) Southern California

Origin time: 060821.9

Epicenter: $\quad 33.95$ N., $118.30 \mathrm{~W}$.

Depth:

Magnitude:

$5 \mathrm{~km}$

2.7 ML

Intensity II: Hawthorne (P), Los Angeles (P), Northern Orange County (press report), Santa Monica (P).

29 April (P) Southern California

Origin time: $\quad 033211.8$

Epicenter: $\quad 33.83 \mathrm{~N}, 117.73 \mathrm{~W}$.

Depth:

Magnitude: $\quad 3.2 \mathrm{ML}, 4.6 \mathrm{mb}(\mathrm{G})$

Intensity III: Santa Ana, Villa Park 
29 April (P) Southern California

origin time: $\quad 040346.2$

Epicenter: $\quad 34.23 \mathrm{~N} ., 116.57 \mathrm{~W}$.

Depth:

$6 \mathrm{~km}$

Magnitude: $\quad 3.8 \mathrm{ML}$

Intensity V: Landers.

Intensity IV: Fawnskin, Lake Arrowhead, Riverside.

Intensity III: Helendale, Orange, Santa Ana.

Intensity II: Anaheim Hills (press report), Big Bear (P), Tustin

(press report).

1 May (P) Southern California

Origin time: $\quad 080254.4$

Epicenter: $\quad 34.37 \mathrm{~N}, 119.97 \mathrm{~W}$.

Depth: $\quad 5 \mathrm{~km}$

Magnitude: $\quad 3.7 \mathrm{ML}$

Intensity IV: Goleta, Santa Barbara.

5 May (P) Baja California

Origin time: 210315.8

Epicenter: $\quad 32.22 \mathrm{~N} ., 115.32 \mathrm{~W}$.

Depth:

$5 \mathrm{~km}$

Magnitude: $\quad 4.8 \mathrm{mb}(\mathrm{G}), 4.1 \mathrm{MS}(\mathrm{G})$,

Intensity IV: $5.5 \mathrm{ML}$

Arizona--San Luis, Yuma.

California--Calexico, Del Mar, Campo, El Centro, Heber, Ocotililo, Potrero, seeley.

Intensity III :

California--Plaster City, San Diego.

6 May (B) Owens Valley region

Origin time: $\quad 10 \quad 14 \quad 14.9$

Epicenter: $\quad 37.47 \mathrm{~N} ., 118.57 \mathrm{~W}$.

Depth:

$37.47 \mathrm{~N}, 118.57 \mathrm{~W}$. Intensity IV:

Intensity III: East San Jose (press report).

11 May (P) Southern California

origin time: $\quad 054732.4$

Epicenter: $\quad 34.00 \mathrm{~N}, 118.35 \mathrm{~W}$.

Depth:

$5 \mathrm{~km}$

Magnitude: $\quad 2.8 \mathrm{ML}$

Intensity III: Culver City, Inglewood, Los Angeles, Santa Monica, Southgate.

11 May (B) Central California origin time: $\quad 115605.6$

Epicenter: $\quad 37.37$ N., $121.76 \mathrm{~W}$.

Depth:

Magnitude:

$3 \mathrm{~km}$

Intensity IV: East San Jose.

11 May (B) Central California

origin time: $\quad 115656.6$

Epicenter: $\quad 37.37 \mathrm{~N}, 121.76 \mathrm{~W}$.

Depth:

Magnitude:

$2 \mathrm{~km}$

Intensity IV: East San Jose.

11 May (B) Central California origin time: $\quad 121811.8$

Epicenter: $\quad 37.38 \mathrm{~N} ., 121.75 \mathrm{~W}$.

Depth :

Magnitude:

$4 \mathrm{~km}$

Intensity IV: East San Jose.

11 May (B) Central California

Origin time: $\quad \begin{array}{lll}12 & 28 & 03.5\end{array}$

Epicenter: $\quad 37.38 \mathrm{~N}, 121.75 \mathrm{~W}$.

Depth:

Magnitude:

$4 \mathrm{~km}$

$3.4 \mathrm{ML}$

Intensity IV: East San Jose.

Magnitude: $\quad 3.7 \mathrm{ML}$

Intensity IV: Bishop.

8 May (B) Central California

origin time: 010702.4

Epicenter: $\quad 36.83 \mathrm{~N}, 120.03 \mathrm{~W}$.

Depth:

$9 \mathrm{~km}$

Magnitude: $\quad 3.3 \mathrm{ML}$

Intensity II: Madera.

8 May (B) Central California

origin time: 065623.3

Epicenter: $\quad 37.37 \mathrm{~N}, 121.75 \mathrm{~W}$.

Depth :

Magnitude: $\quad 3.4 \mathrm{ML}$

11 May (P) Southern California

Origin time: $\quad 175740.6$

Epicenter: $\quad 34.00 \mathrm{~N}, 118.45 \mathrm{~W}$.

Depth:

Magnitude: $\quad 2.9 \mathrm{M}$

Intensity III: Beverly Hills, Inglewood, Santa Monica.

22 May (B) Central California

origin time: $\quad 215606.9$

Epicenter: $\quad 36.64 \mathrm{~N}, 121.31 \mathrm{~W}$.

Depth: $\quad 4 \mathrm{~km}$

Magnitude: $\quad 3.7 \mathrm{mb}(\mathrm{G}), \quad 3.7 \mathrm{ML}$

Intensity II: Hollister, Stone

Canyon.

This earthquake is the beginning of a swarm along the Caleveras fault northeast of San Jose. $\frac{\text { Intensity III }}{\text { report). }}$ East San Jose (press

10 May (B) Central California

origin time: $\quad 155540.5$

Epicenter: $\quad 37.38$ N., $121.75 \mathrm{~W}$.

Depth :

$2 \mathrm{~km}$

Magnitude:

$3.3 \mathrm{ML}$

22 May (B) Northern California

Origin time: $\quad 220158.2$

Epicenter: $\quad 36.64 \mathrm{~N} ., 121.31 \mathrm{~W}$.

Depth:

Magnitude:

$5 \mathrm{~km}$

Intensity II: Hollister (B), Stone

Canyon (B).

23 May (P) Southern California

Origin time: 091650.8

Epicenter: $\quad 33.90 \mathrm{~N}, 119.17 \mathrm{~W}$.

Depth:

$5 \mathrm{~km}$ 
Cal ifornia--Continued

Magnitude: $\quad 4.0 \mathrm{mb}(\mathrm{G}), 3.9 \mathrm{ML}$

Intensity IV: Compton, Hollywood Beach (press report), oxnard (press report), Port Hueneme (press report), Silver Strand Beach. Intensity III: Glendale. Intensity II: Point Mugu.

27 May (B) Northern California origin time: $\quad 182241.3$

Epicenter: $\quad 38.54 \mathrm{~N} ., 122.74 \mathrm{~W}$. Depth:

Magnitude:

$9 \mathrm{~km}$

Intensity II: Santa Rosa (B), Sonoma county (B).

31 May (P) Imperial Valley region Origin time: 051649.2

Epicenter: $\quad 32.87 \mathrm{~N}, 115.48 \mathrm{~W}$.

Depth:

Magnitude:

$10 \mathrm{~km}$

Intensity II: Brawley.

31 May (P) Imperial Valley region

oriqin time: $\quad 114445.2$

Epicenter: $\quad 32.87$ N., $115.48 \mathrm{~W}$.

Depth:

Magnitude: $\quad 2.9 \mathrm{ML}$

Intensity II: Brawley.

4 June (P) Southern California

Origin time: 035717.3

Epicenter: $\quad 33.92 \mathrm{~N} ., 117.83 \mathrm{~W}$.

Depth :

Magnitude:

$6 \mathrm{~km}$

3. $5 \mathrm{ML}$

This earthquake was felt over an area of approximately $1,800 \mathrm{sq} \mathrm{km}$ ( $\mathrm{fig}$. 8).

Intensity V: Whittier (light furniture and small objects shifted).

Intensity IV: Anaheim, Arcadia, Atwood, Big Bear City, Bonsall, Brea, Buena Park, Burbank, Costa Mesa, Covina, Cudahy, Cypress, Diamond Bar, Downey, El Segundo, Fawnskin, Fountain Valley, Fullerton, Garden Grove, Gardena, Glendale, Hancock, Ladera Heights, La Habra, Long Beach, Los Angeles, Maywood, Midway City, Mon rovia, Monterey Park, Montrose, Murrieta, Norwalk, Paramount, Pasadena, Placentia, Pomona, San Jacinto, Sierra Madre, South Gate, Stanton, sunset Beach, Vista, West Covina, Westminster, Wildomar, Yorba Linda.

Intensity III: Glendora, Glenoaks, Los Alamitos, Santa Ana.

Intensity II: Altadena, Green Valley Lake, Huntington Beach, Montclair, Phelan, San Gabriel, Van Nuys .

5 June (P) Southern California

origin time: $\quad 160303.8$

Epicenter: $\quad 33.42 \mathrm{~N} .116 .70 \mathrm{w}$

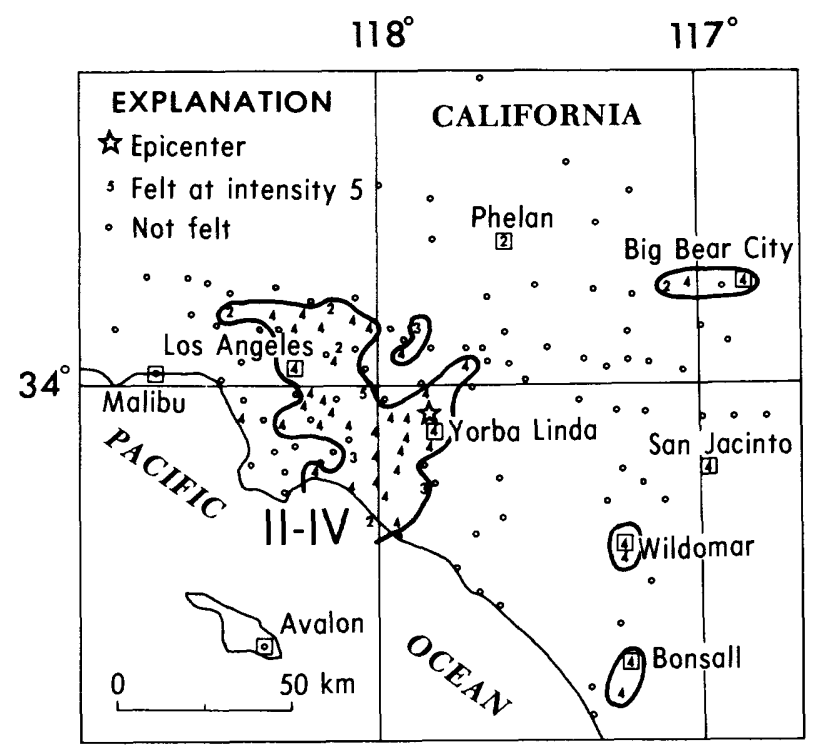

FIGURE 8.--Isoseismal map for the southern California earthquake of 4 June $1978,035717.3$ UTC. Roman numerals represent Modified Mercalli intensities between isoseismals; Arabic numerals are used to represent these intensities at specific sites.

\section{California--Continued}

Depth: $12 \mathrm{~km}$

Magnitude:

$3.9 \mathrm{mb}(\mathrm{G}), 4.4 \mathrm{ML}$

This earthquake was felt over an area of approximately $11,000 \mathrm{sq} \mathrm{km} \mathrm{(fig.}$ 9).

Intensity V: Anza (hairline cracks in exterior brick walls, small objects shifted), Winchester (few cracked windows; windows, doors, and dishes rattled).

Intensity IV: Aguanga, Alpine, Banning, Bonita, Borrego Springs, Camp Pendleton, El Cajon, Escondido, Guatay, Hemet, Idyllwild, Indio, Jamul, Julian, La Mirada, Laguna Niquel, Lakeside, North Palm Springs, Pala, Palm Springs, Palomar Mountain, Pauma Valley, Pico Rivera, Pine Valley, Pomona, Ramona, Ranchita, San Diego, San Diego NAB, San Marcos, Santa Ysabel, Valley Center, Vista, Warner Springs.

Intensity III: Boulevard, Del Mar, La Jolla, Mountain Center, North Shore, Palm Desert, Pico Rivera, Rincon Springs (press report), San Diego--Lindbergh Field, South Whittier.

Intensity II: Lake Elsinore, Mt. Baldy. 


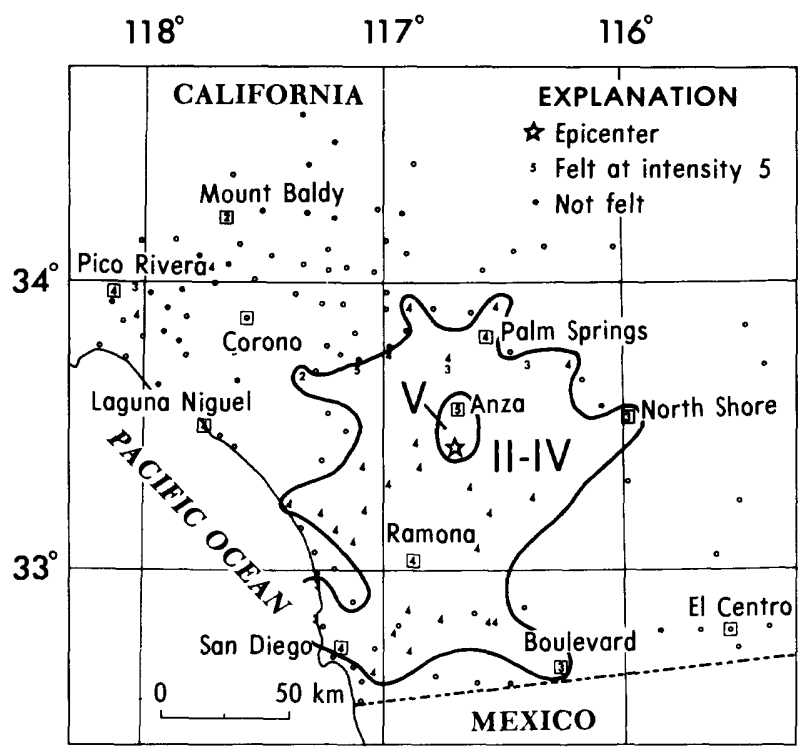

FIGURE 9.--Isoseismal map for the southern California earthquake of 5 June $1978,160303.8$ UTC. Roman numerals represent Modified Mercalli intensities between isoseismals; Arabic numerals are used to represent these intensities at specific sites.

California--Continued

11 June (B) Northern California

Origin time: $\quad 2012 \quad 03.9$

Epicenter: $\quad 41.44 \mathrm{~N} ., 121.89 \mathrm{~W}$.

Depth: $2 \mathrm{~km}$

Magnitude: $\quad 3.5 \mathrm{ML}$

Intensity II: Mt. Shasta area (B).

12 June (B) Northern California

Origin time: 091844.1

Epicenter: $\quad 41.46 \mathrm{~N} .121 .86 \mathrm{~W}$.

Depth:

Magnitude:

$2 \mathrm{~km}$

$3.8 \mathrm{ML}$

Intensity II: Mt. Shasta area (B).

14 June (P) Southern California

Origin time: $\quad 042749.4$

Epicenter: $\quad 34.38 \mathrm{~N} ., 118.63 \mathrm{~W}$.

Depth:

$6 \mathrm{~km}$

Magnitude: $2.9 \mathrm{ML}$

Intensity II: Newhall (P).

16 June (P) Central California

origin time: 042131.9

Epicenter:

Depth:

Magnitude:

$35.03 \mathrm{~N} ., 119.13 \mathrm{~W}$.

$5 \mathrm{~km}$

$4.1 \mathrm{mb}(\mathrm{G}), 4.1 \mathrm{ML}$, $4.4 \mathrm{ML}(\mathrm{B})$

Intensity $\mathrm{V}$ : Mettler (small

objects overturned), Pumpkin Center (furniture shifted), Tupman (small objects fell).

Intensity IV: Fellows, Lebec, Taft. Intensity III: Frazier Park,
California--Continued

Shafter.

Intensity II: Buena Vista pumping plant (P), New Cuyama, Santa Barbara (P), Saugus.

25 June (P) Southern California

Origin time: $\quad 073111.4$

Epicenter: $\quad 34.05$ N., $117.27 \mathrm{~W}$.

Depth :

Magnitude:

$5 \mathrm{~km}$

Intensity II: Fontana (P).

2 July (B) Northern California

Origin time: $\quad 115757.0$

Epicenter: $\quad 36.90 \mathrm{~N} ., 122.18 \mathrm{~W}$.

Depth:

Intensity V: Capitola (small

objects fell), Watsonville (small objects fell).

Intensity IV: Aptos, Boulder Creek, Davenport, East Santa Cruz, Felton, Mount Herman, Pescadero, Salinas, Santa Cruz.

Intensity II: Brookdale, Santa Clara.

5 July (P) Southern California

Origin time: $\quad 104755.6$

Epicenter: $\quad 33.88$ N., $116.50 \mathrm{~W}$.

Depth:

Magnitude: $\quad 3.8 \mathrm{ML}$

Intensity $V: \quad$ North Palm springs (small objects fell, few windows cracked, light furniture shifted). Intensity IV: Banning, Desert Hot Springs (press report), Indio, Landers, Palm Springs.

$18 \mathrm{July}$ (B) Central California

Origin time: $\quad 190903.7$

Epicenter: $\quad 36.99$ N. $121.67 \mathrm{~W}$.

Depth: $\quad 5 \mathrm{~km}$

Magnitude: $\quad 2.9 \mathrm{ML}$

Intensity IV: Corralitos.

Intensity III: Watsonville.

21 July (P) Southern California

Origin time: $\quad 132441.9$

Epicenter: $\quad 34.05 \mathrm{~N}, 118.90 \mathrm{~W}$.

Depth :

Magnitude.

Intensity III: Newbury Park (P), Redondo Beach (P).

$23 \mathrm{July}$ (B) Northern California

Origin time: 073335.6

Epicenter: $\quad 39.40 \mathrm{~N}, 121.46 \mathrm{~W}$.

Depth:

Intensity III: Nevada City, Oroville.

23 July (B) Central California

origin time: $\quad 143842.4$

Epicenter: $\quad 35.93 \mathrm{~N} ., 120.51 \mathrm{~W}$.

Depth: 
Magnitude: $\quad 3.2 \mathrm{ML}$

Intensity II: Bradley.

26 July (P) Southern California

origin time: 003853.6

Epicenter: $\quad 34.35 \mathrm{~N} ., 116.92 \mathrm{~W}$.

Depth :

Magnitude:

$6 \mathrm{~km}$

Intensity IV: Apple Valley, Big Bear City, Big Bear Lake, Green Valley Lake.

Intensity III: Fawnskin, Lucerne Valley, San Bernardino.

31 July (B) Northern California

origin time:

$09 \quad 15 \quad 44.3$

Epicenter:

$37.71 \mathrm{~N}, 122.14 \mathrm{~W}$.

Depth:

Magnitude:

$9 \mathrm{~km}$

$3.0 \mathrm{ML}$

Intensity IV: Danville (press report).

Intensity III: Castro Valley (press report), Hayward (press report), Lafayette (B), Oakland (B), San Leandro (press report), San Lorenzo (press report).

1 August (B) Northern California

Origin time: 090234.5

Epicenter: $\quad 41.45 \mathrm{~N}, 121.88 \mathrm{~W}$.

Depth:

$2 \mathrm{~km}$

Magnitude:

$4.5 \mathrm{mb}(\mathrm{G}), 4.6 \mathrm{ML}$

This is the largest event in a series of earthquakes which occurred in this area over a period of several months, six of which were felt within the following 7 hours. The magnitude 4.3 event at $09 \quad 46 \quad 44.6$ was felt at about the same intensity as this one, but because it occurred only 44 minutes later, the intensity data for the separate events could not be differentiated. Most of these earthquakes that were over magnitude 3.0 were felt by Forest Service personnel. The intensities 1 isted below are associated with this event, the largest in the series, but may also include data from the following event at 09 4644.6 .

Surface fractures were associated with this activity. The ruptures occurred along the Stephens Pass fault (Bennett and others, 1979), a 2-km-long fault trending north from a point $260 \mathrm{~m}$ south of stephens Pass Road, at a point about $31 \mathrm{~km}$ northeast of McCloud.

Intensity V: Dunsmuir (heavy furniture shifted).

Intensity IV: Bieber, Rig Bend, Dorris, Edgewood, Etna, Gazelle, Klamath River, Macdoel, McCloud, Mount Hebron, Mount Shasta, Scott
Bar, Tennant, Weed.

3 August (P) Southern California

Origin time: 043042.1

Epicenter: $\quad 33.67 \mathrm{~N}, 116.70 \mathrm{~W}$.

Depth:

Magnitude:

$5 \mathrm{~km}$

Intensity II: Idyllwild (P).

11 August (P) Southern California

Origin time: 004730.1

Epicenter: $\quad 34.15 \mathrm{~N}, 117.45 \mathrm{~W}$.

Depth:

Magnitude:

$4 \mathrm{~km}$

4. $0 \mathrm{ML}$

Intensity IV: Arlington, Blooming-

ton, Brea, Bryn Mawr, Colton, Compton, Crestline, Del Rosa, Etiwanda, Fawnskin, Fontana, Glendale, Lake Arrowhead, Lakewood, Llano, Loma Linda, Los Angeles, Mount Baldy, Norton AFB, Pacific Palisades, Redlands, Rimforest, Riverside, Rubidoux, San Bernardino, Santa Fe Springs, Sunnymead, Wrightwood.

Intensity III: Bellflower, Canoga Park, Cedarpines Park, Leona Valley, Northridge, Palm Springs, Pasadena (press report), Sylmar, Westside.

Intensity II: Blue Jay, El Toro Marine Air station, Montrose, ontario, Saugus, Toluca Lake.

13 August (B) Northern California

origin time: 055548.0

Epicenter:

Depth :

$41.43 \mathrm{~N} ., 121.89 \mathrm{~W}$.

Magnitude:

$4.3 \mathrm{mb}(\mathrm{G}), 4.1 \mathrm{MS}(\mathrm{G})$, $4.3 \mathrm{ML}$

Intensity IV: Horse Creek, McCloud, Weed.

Intensity II: Forks of Salmon.

13 August (K) Southern California

Origin time: 225452.4

Epicenter:

Depth :

Magnitude:

$34.37 \mathrm{~N}, 119.72 \mathrm{~W}$. $12 \mathrm{~km}$

$5.5 \mathrm{mb}(\mathrm{G}), 5.6 \mathrm{MS}(\mathrm{G})$, $5.1 \mathrm{ML}, 5.7 \mathrm{ML}(\mathrm{B})$

The damage described below was taken from a report by Miller and Felszeghy, 1978 .

About 65 people were injured, but there were no fatalities. The worst damage (intensity VII) occurred at the University of California Santa Barbara (UCSB) campus, at Goleta, and at Santa Barbara. At the UCSB campus several of the multi-story, reinforced-concrete structures sustained moderate diagonal cracking of the shear walls in the lower stories. Instruments and supplies were destroyed in some laboratories. Damage occurred to 
light fixtures, ceilings, and plaster throughout the campus. Similar but less severe damage occurred in the commercial district of Goleta and in the Santa Barbara area. The damage loss is estimated at $\$ 7.31$ million.

A Southern Pacific Transportation Company freight-train derailment occurred west of Goleta near winchester Canyon Road in an area of cut-and-fill roadbed (fig. 10 ). The 49 empty and 9 loaded cars travelling at about $50 \mathrm{mph}$ derailed when passing over a "kink" in the tracks, apparently the result of roadbed-fill failure; 30 of the cars were derailed and a section of the track was damaged.

A total of three overpasses crossing U.S. Highway 101, all steelreinforced concrete structures located in the Goleta area, suffered significant earthquake damage. These overpasses are: the adjacent curving bridges on ward Memorial Road, the one at Glen Annie Road, and the one at the western end of Hollister Avenue just east of the railroad derailment. The most extensive damage was sustained by the Ward Memorial bridges, which were subjected to considerable transverse motion that shifted the superstructures relative to the abutments causing the concrete to crack and spall in a number of places. Most of the Ward Memorial Road bridges' bents sustained damage that ranged from light cracking and incipient spalling, to localized deep spalling that exposed reinforcement bars.

The earthquake caused several rock slides on San Marcos Pass Road, the section of State Highway 154 that runs northwest through the Santa Ynez Mountains from U.S. Highway 101 between Goleta and Santa Barbara. Most of the slides occurred on roadcuts where there were steep inclines. Extension fractures opened in at least one area on a steep slope which forms the shoulder of the highway. Some minor slides occurred along the cliffs at the coastline and off the roads in the Santa Ynez Mountains.

Porcella and others (1978) reported that eight accelerograms were recovered from strong-motion stations at Santa Barbara, Goleta, Cochuma Dam, and the UCSB campus. The highest accelerations that were recorded at the three-story North Hall building on the UCSB campus were $0.44 \mathrm{~g}, 0.66 \mathrm{~g}$, and $0.99 \mathrm{~g}$, for the ground, third floor, and roof levels, respectively.

Due to an error, the affected area of this earthquake was insufficiently canvassed with USGS questionnaires; as a result, the isoseismal map shown in figure 11 does not have as much intensity data as would normally be collected. However, the data is extensive enough to show this earthquake was felt over an area of approximately $25,000 \mathrm{sq} \mathrm{km}$ along the southern California coast.

\section{Intensity VII:}

Goleta-University of California Santa Barbara area--There was significant earthquake damage to buildings within an $8-\mathrm{km}$ radius of the UCSB campus. On campus there was extensive cracking of reinforced-concrete shear walls in the Biological Sciences II Building, Engineering Building, Library III, University Center, Anacapa Residence Hall, Santa Cruz Residence Hall, and North Hall. Mechanical equipment on or near the roof tops of multistory buildings suffered considerable damage from building movement during the earthquake. A few very old adobe or wood-frame buildings were seriously damaged.

The Air Traffic Control Tower at the airport, a steel-frame structure with 1 ateral reinforcment, was shaken so that it sustained an estimated permanent deflection of 1.3 to 2.5 $\mathrm{cm}$. Two other buildings sustained cracked concrete-floor slabs, with shifted wood columns and walls in one. Damage to the hangars consisted of buckled diagonal steel bracings and failure of some of the structural connections at the supports for the large sliding doors.

The Ward Memorial Road bridges were damaged by the shifting of the ground, which caused the bridge decks to impact at several expansion joints and resulted in cracked and spalled concrete. The bridges were temporarily closed.

The most common damage to residential and small commercial buildings consisted of 


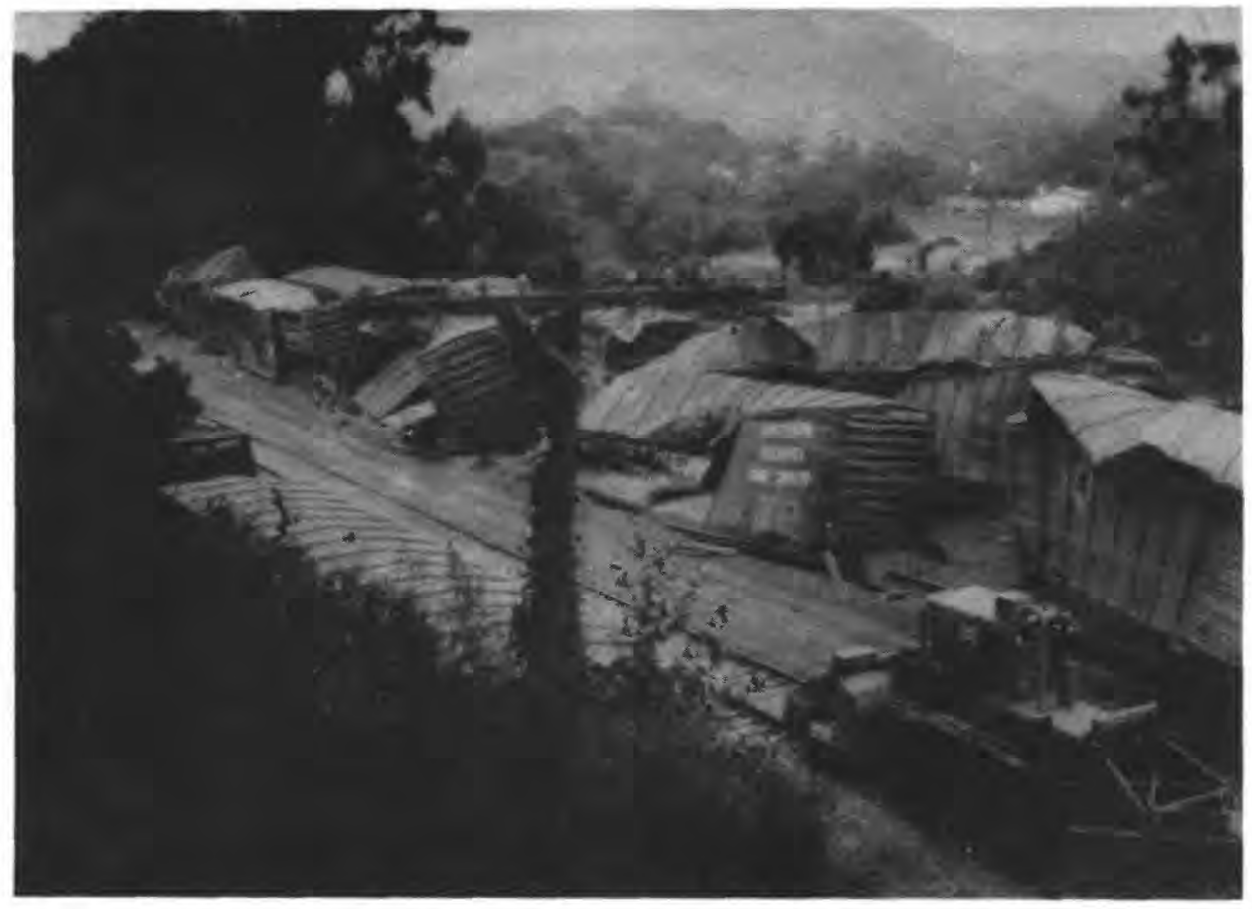

FIGURE 10.--Southern Pacific Transportation Company freight train derailment west of Goleta, Calif.

\section{California--Continued}

cracked and fallen plaster and stucco, differential settlement of foundations, failure of unreinforced chimneys, fallen hot water heaters, dislodgement of air-conditioning ducts, dislodgement of tiles and panels from suspended acoustical ceilings, lighting fixtures loosened or fallen, and glass broken. A common type of damage to mobile homes is shown in figure 12. The mobile homes were thrown off their mounts, crumpling the skirts around the base and dislodging the contents of the home.

The most widespread type of damage was breakage of household belongings and store merchandise that toppled from shelves. In homes, cupboards and refrigerators were thrown open, spilling their contents. Liquor stores and supermarkets sustained heavy losses from breakage. About one-third of the UCSB 1 ibrary's 1.2 million books were thrown to the floor. The UCSB laboratories suffered much damage and glass breakage when wall cabinets broke loose and fell, spilling their contents.

\section{California-continued}

buildings sustained diagonal cracks in their reinforcedconcrete shear walls, particularly in the lower floors. The Freitas Building at 200 E. Carrillo Street and the Santa Barbara Court House Building suffered some damage. The Santa Barbara County Administration Building at Anacapa and Anapamu Streets suffered diagonal tension cracks in some of the columns on the north side of the building. The roof of an unoccupied restaurant that was being remodeled at $100 \mathrm{~W}$. Anapamu Street collapsed. The floating dock at Marina No. 1 was damaged by the movement of the concrete support piles which were buried $3.6 \mathrm{~m}$ into the mud. Several water mains were broken. There was widespread breakage and damage to household belongings or store merchandise as described for the Goleta area above.

Intensity VI: Solvang (cracked drywall, hairline cracks in exterior walls, stone or brick fences cracked, few windows cracked, small objects shifted, vehicles rocked moderately, felt by all).

Intensity V: Carpenteria, Los Alamos, Santa Maria, Santa Ynez. 


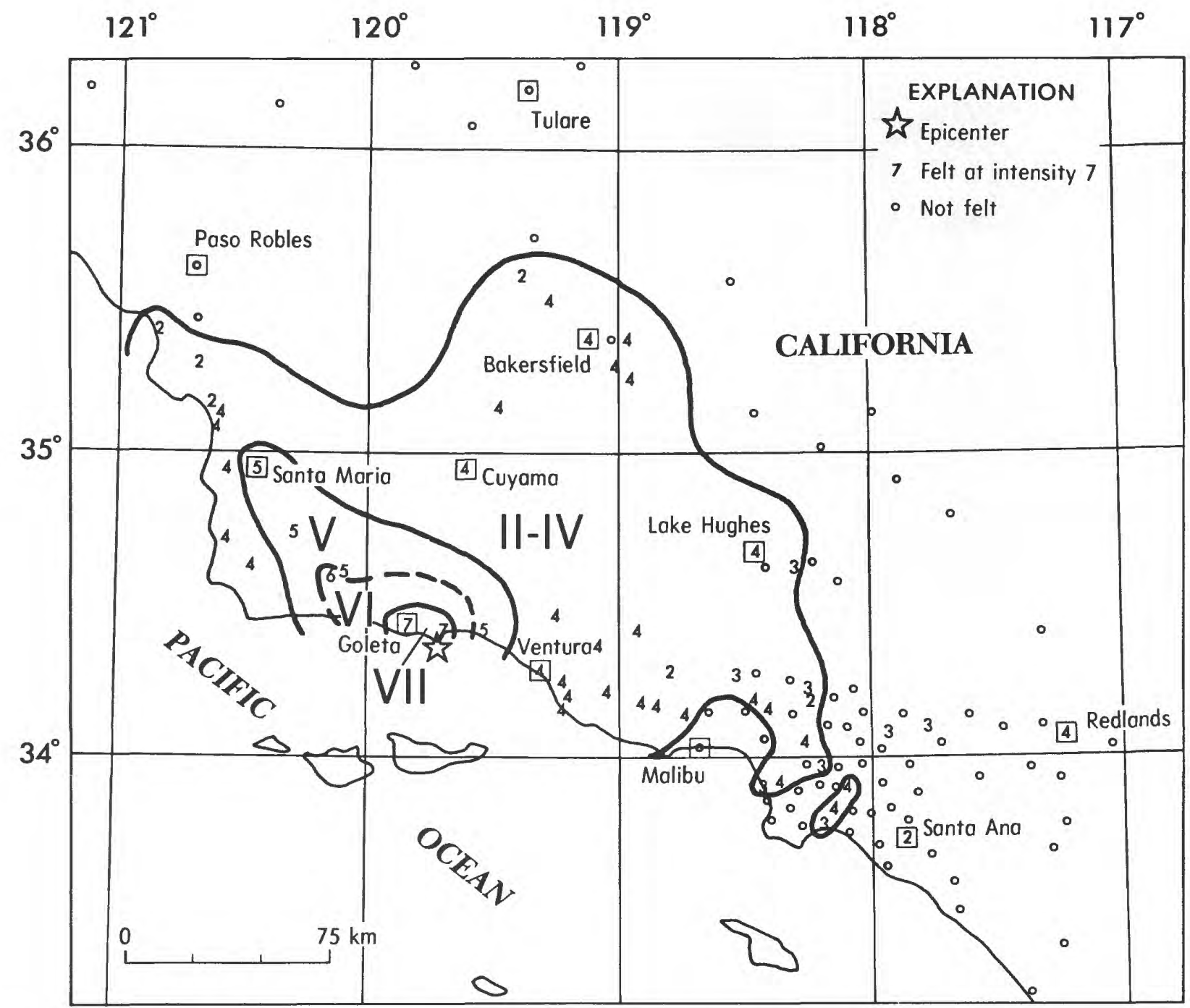

FIGURE 11.--Isoseismal map for the southern California earthquake of 13 August 1978 , 225453.5 UTC. Roman numerals represent Modified Mercalli intensities between isoseismals: Arabic numerals are used to represent these intensities at specific sites.

California--Continued

Intensity IV: Agoura, Arroyo

Grande, Artesia, Bakersfield, Camarillo, Cuyama, East Ventura, Fillmore, Grover City, Guadalupe, Halcyon, Hawthorne, Hillcrest Center, Lake Hughes, Lakewood, Lamont, Lompoc, Montalvo, Newbury Park, Norwalk, Oxnard, Palms, Pineside, Port Hueneme, Pumpkin Center, Oceano, Ojai, Redlands, Santa Paula, Shafter, Sherman Oaks, Studio City, Taft, Thousand Oaks, Van Nuys, Vandenburg AFB, Ventura.

Intensity III: Bellflower, Granada Hills, La Crescenta, La Verne, Leona Valley, Long Beach, Manhattan Beach, West Covina.

\section{California--Continued}

Pismo Beach, San Luis Obispo, Santa Ana (Marine Corps Air station), Simi Valley, Wasco.

13 August (K) Southern California Origin time: 231101.7 Epicenter: $\quad 34.40 \mathrm{~N}$. $119.76 \mathrm{~W}$. Depth: $13 \mathrm{~km}$ Magnitude: $\quad 3.4 \mathrm{ML}(\mathrm{P}), 3.3 \mathrm{ML}$ Intensity II: Santa Barbara area.

13 August (K) Southern Cal ifornia Origin time: 231502.5 Epicenter: $\quad 34.41 \mathrm{~N} ., 119.77 \mathrm{~W}$. Depth: $13 \mathrm{~km}$

Magnitude: $\quad 3.1 \mathrm{ML}(\mathrm{P}), 3.0 \mathrm{ML}$ 


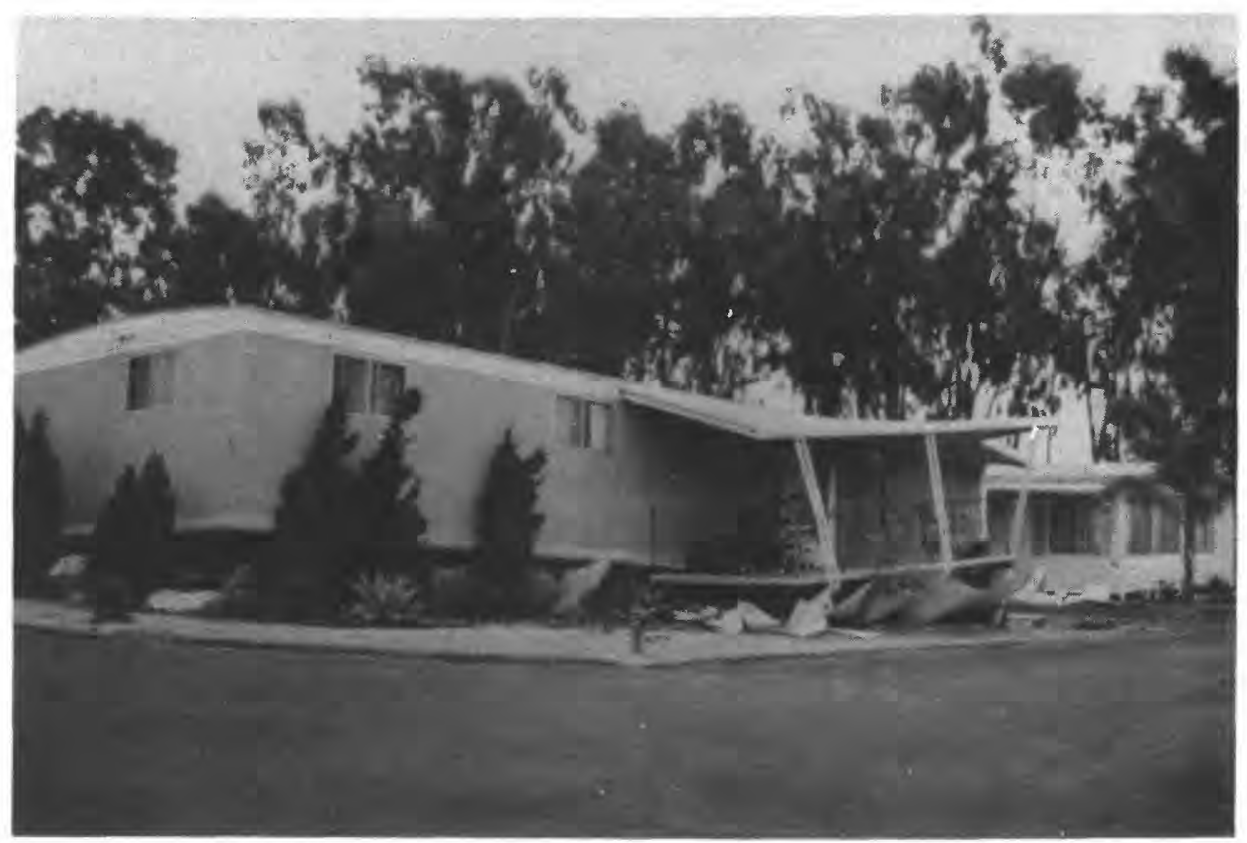

FIGURE 12.--Damage to mobile home near Goleta, Calif.

California--Continued

13 August (K) Southern California

Origin time: $\quad 232353.8$

Epicenter: $\quad 34.40 \mathrm{~N}, 119.74 \mathrm{~W}$.

Depth:

Magnitude:

Intensity II: Santa Barbara area.

14 August (K) Southern California

Origin time: 010235.1

Epicenter: $\quad 34.40 \mathrm{~N}, 119.72 \mathrm{~W}$.

Depth:

Magnitude: $\quad 3.1 \mathrm{ML}(\mathrm{P}), 2.7 \mathrm{ML}$

Intensity II: Santa Barbara area.

16 August (B) Northern California

Origin time: 074532.4

Epicenter: $\quad 40.33 \mathrm{~N} ., 124.39 \mathrm{~W}$.

Depth:

Magnitude:

$23 \mathrm{~km}$

$3.8 \mathrm{ML}$

Intensity IV: Fortuna, Rio Dell.

Intensity III: Eureka, Ferndale, Freshwater, Scotia.

16 August (K) Southern California

Oriqin time: $\quad 133511.6$

Epicenter: $\quad 34.41 \mathrm{~N} ., 119.80 \mathrm{~W}$.

Depth:

Magnitude: $\quad 3.5 \mathrm{ML}(\mathrm{P}), 3.2 \mathrm{ML}$

Intensity IV: Goleta (University of

California Santa Barbara campus),

Los Olivos, Oxnard, New Cuyama,

Santa Barbara.

Intensity II: Ventura.

19 August (P) Baja California

Origin time: 093107.3

Epicenter: $\quad 32.42 \mathrm{~N}, 116.83 \mathrm{~W}$

Depth:
Cal ifornia--Continued

Intensity V: Tecate (1 ight furniture shifted; small objects

shifted; windows, doors, and dishes rattled, few awakened).

Intensity IV: Alpine, Bonita, Bostonia, Boulevard, Campo, Chula Vista, Dulzura, El Cajon, Guatay, Imperial Beach, Jamul, La Jolla, Lakeside, La Mesa, Lemon Grove, Pine Valley, Ramona, San Diego, San Diego (Lindbergh Field), San Diego (Montgomery Field), Santee, University City.

Intensity II: San Ysidro.

28 August (B) Central California

origin time: 033218.4

Epicenter: $\quad 37.55 \mathrm{~N}, 121.86 \mathrm{~W}$.

Depth: $\quad 11 \mathrm{~km}$

Magnitude: $\quad 2.8 \mathrm{ML}$

Intensity IV: Fremont, Mountain

View, Warm Springs (all reported windows, doors, and dishes rattled; buildings creaked or trembled). Intensity III: San Leandro.

Intensity II: Livermore (press report), Pleasanton, Redwood, Walnut Creek (press report).

29 August (B) Northern California

Origin time: $\quad 001446.4$

Epicenter: $\quad 37.36 \mathrm{~N}, 121.72 \mathrm{~W}$.

Depth:

Magnitude:

$8 \mathrm{~km}$

$4.1 \mathrm{ML}$

Intensity VI: San Jose--east side of the city (acoustical ceiling tiles fell in an East Side supermarket; at 3720 Sierra Road cracks 
California--Continued

and the walls were 1 ifted $1.7 \mathrm{~cm}$ from the floor; in Eastridge a small amount of glassware was broken--press report).

Intensity V: Hayward (small objects broken; windows, doors, and dishes rattled), Mount Hamilton-Lick Observatory (small cracks in dry-wall, hairline cracks in exterior walls), San Jose (Cambrian Park--small objects overturned).

Intensity IV: Boulder Creek, Burlingame, Felton, Moffett Field NAS, Mountain View, Oakland, Santa Clara, Sunnyvale, Union City, Vallejo.

Intensity III: Fremont (B), Milpitas, Pacifica.

Intensity II: Alameda, Belmont, Livermore (press report), Newark (press report), Santa Cruz, Stockton (Airport Control Tower).

29 August (B) Northern California

origin time: 001845.3

Epicenter: $\quad 37.35 \mathrm{~N} ., 121.72 \mathrm{~W}$.

Depth:

Magnitude:

$7 \mathrm{~km}$

$3.8 \mathrm{ML}$

Intensity IV: Mount Hamilton--Lick observatory.

Intensity III: Fremont (B), San Jose (press report), San Jose (Cambridge Park).

29 August (P) Southern California

Origin time: $\quad 060449.8$

Epicenter: $\quad 34.38 \mathrm{~N} ., 119.77 \mathrm{~W}$.

Depth: $\quad 5 \mathrm{~km}$

Maqnitude: $\quad 2.8 \mathrm{ML}$

Intensity II: Santa Barbara (P).

29 August (P) Southern California

origin time: $\quad 105146.0$

Epicenter: $\quad 34.38 \mathrm{~N}, 119.80 \mathrm{~W}$.

Depth:

Magnitude:

$5 \mathrm{~km}$

2. $5 \mathrm{ML}$

Intensity II: Santa Barbara (P).

1 September (B) Northern California

Origin time: 093125.4

Epicenter: $\quad 37.34 \mathrm{~N}, 121.78 \mathrm{~W}$.

Depth: $\quad 8 \mathrm{~km}$

Magnitude: $\quad 3.3 \mathrm{ML}$

Intensity II: San Jose (B).

3 September (P) Southern California

origin time: $\quad 181046.5$

Epicenter: $\quad 33.95 \mathrm{~N} ., 117.72 \mathrm{~W}$.

Depth:

Magnitude:

$6 \mathrm{~km}$

Intensity IV: La Puente, Pomona,

Riverside, Yorba Linda.

Intensity III: Los Serranos.

Intensity II: Anaheim (press

report), Chino, Di amond Bar, East

Los Angeles County (P), North

Orange County (P), Redlands, South
California--Continued

4 september (B) Lake Tahoe region

origin time:

$04 \quad 52 \quad 32.3$

Epicenter:

Depth:

Magnitude:

$38.82 \mathrm{~N} ., 119.81 \mathrm{~W}$.

$18 \mathrm{~km}$

$3.9 \mathrm{mb}(\mathrm{G}), 4.6 \mathrm{ML}$

Only the Topaz and eastern Amador County intensity data are from USGS questionnaires, all the other intensities are based on press reports.

Intensity $V$ :

California--South Lake Tahoe (few broken dishes).

Intensity IV:

California--Eastern Amador County, in the Lake Tahoe Sierra region.

Nevada--Gardnerville, stateline, Topaz.

Intensity III:

California-Grass Valley, Ione, Jackson, Leek Springs (El Dorado National Forest), Placerville, Pollock Pines, Tahoe City, Truckee.

Nevada--Carson City, Incline Villaqe, Minden, Zephyr Cove.

Intensity II:

California--Stockton, Strawberry.

4 September (B) Lake Tahoe region

origin time: $\quad 215453.2$

Epicenter: $\quad 38.81 \mathrm{~N} .119 .82 \mathrm{~W}$.

Depth:

Magnitude: $\quad 4.7 \mathrm{mb}(\mathrm{G}), 5.3 \mathrm{ML}$

This is the largest magnitude event of a series of earthquakes on september 3 and 4 in the area south of Lake Tahoe. It was felt over an area of approximately 45,500 sq km of California and Nevada (fig. 13).

Intensity VI:

California--Mt. Aukum (dry wall cracked, hairline cracks in exterior walls, water splashed onto sides of lakes and pools).

Nevada--Genoa (plaster and dry wall cracked; hairline cracks in exterior walls; windows, doors, and dishes rattled; vehicles rocked slightly; buildings shook).

Intensity $\mathrm{V}$ :

California--Bear Valley (heavy furniture shifted; small objects shifted; pictures fell; buildings shook; windows, doors, and dishes rattled), Camp Connell (light furniture shifted; small objects shifted; hanging pictures swung; buildings shook; windows, doors, and dishes rattled), Glencoe (heavy furniture shifted; hanging pictures swung; buildings shook; windows, doors, and dishes rattled), South Lake Tahoe (small 


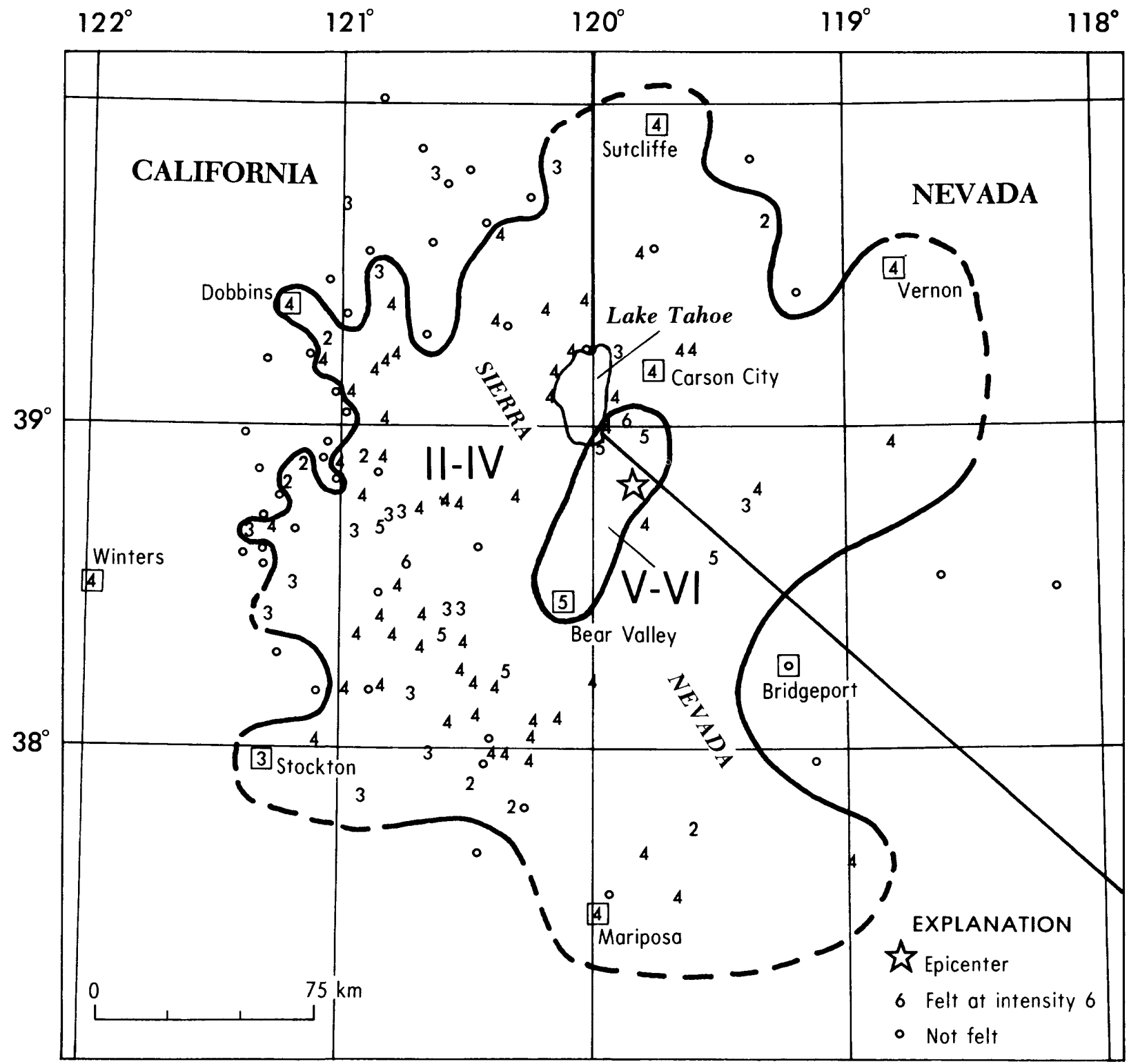

FIGURE 13.--Isoseismal map for the Lake Tahoe earthquake of 4 September 1978 , 2154 53.2 UTC. Roman numerals represent Modified Mercalli intensities between isoseismals; Arabic numerals are used to represent these intensities at specific sites.

\section{Cal ifornia--Continued}

cainers slightly disturbed; buildings shook; windows, doors, and dishes rattled), Topaz (small objects fell, buildings shook; windows, doors, and dishes rattled). Twin Bridges (light furniture shifted; small objects fell; hanging pictures swung; buildings shook; windows, doors, and dishes rattled).

Nevada--Minden ( light furniure shifted; small objects overturned, fell, and broke; water in

\section{California--Continued}

small containers spilled; hanging pictures swung out of place; buildings shook; windows, doors, and dishes rattled). Intensity IV:

California--Alta, Altaville, Amador City, Angels Camp, Arnold, Baxter, Camino, Carnelian Bay, Chicago Park, Citrus Heights, Coleville, Colfax, Cool, Diamond Springs, Dobbins, Douglas Flat, Echo Lake area, El portal, Fiddletown, Floriston, Foresthill, 
Georgetown, Gold Run, Hathaway Pines, Homewood, Ione, Jackson, Kyburz, Linden, Long Barn, Lotus, Mammoth Lakes, Mariposa, Markleville, Mi-Wuk Village, Mokelumne Hill, Mono Vista, Mountain Ranch, Murphys, Pacific House, Penn Valley, Pine Grove, Pollock Pines, Rail Road Flat, River Pines, Sheep Ranch, Sierraville, Soda Springs, Sonora, Standard, Stateline (press report), Strawberry, Sutter Creek, Tahoe City, Tahoe Vista, between Tamarak Lake and Echo Lake, Truckee, Tuolumne, Twain Harte, Valley Springs, Wallace, Washington, Wawona, White Pines, Winters.

Nevada--Carson City, Dayton, Fallon, Gardnerville, Glenbrook, Reno, Schurz, Silver City, Smith, zephyr Cove.

Intensity III:

California--Alleghany, Blairsden, Chilcoot, Copperopolis, Grass Valley (press report), La Porte, Pioneer, Placerville (press report), Sacramento (Foothill Farms), San Andreas, Shingle Springs, Sloughhouse, Smithflat, Stockton, Valley Home, Volcano, West Point, wilton.

Nevada--Incline Village, Wellington. Intensity II:

California--Avery, Chinese Camp, Dutch Flat, Greenwood, Loomis, Moccasin, Nevada City, Newcastle, Yosemite Lodge.

Nevada--Wadsworth.

8 September (B) Northern California

origin time: $\quad 165947.8$

Epicenter: $\quad 38.64 \mathrm{~N}, 121.91 \mathrm{~W}$.

Depth :

$17 \mathrm{~km}$

Magnitude: $\quad 4.4 \mathrm{mb}(\mathrm{G}), 4.2 \mathrm{ML}$

This earthquake was felt over an area of approximately $4,500 \mathrm{sq} \mathrm{km}$ of northern California (fig. 14).

Intensity $V:$ Madison (light furniture and small objects shifted; windows, doors, and dishes rattled; buildings shook; pictures swung), North Highlands (few windows cracked; windows, doors, and dishes rattled).

Intensity IV: Brooks, Capay, Citrus Heights, College City, Davis, Dixon, Esparto, Liberty Farms, Rescue, Sacramento, Steele Park, Vacaville, Winters (press report), Woodbridge, Woodland, Yolo, Zamora.

Intensity III: Courtland, Fairfield (press report), Loma Mar, Napa (press report), Robbins, Sonoma (press report), Yountville.

Intensity II: Benecia, Cobb

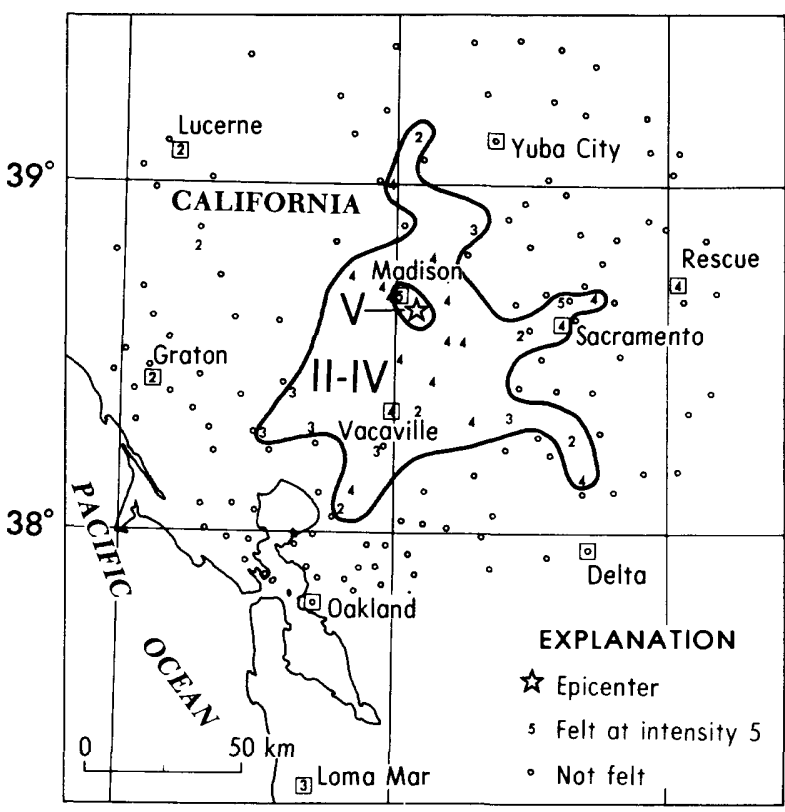

FIGURE 14.--Isoseismal map for the northern California earthquake of 8 September $1978,165947.8$ UTC. Roman numerals represent Modified Mercalli intensities between isoseismals; Arabic numerals are used to represent these intensities at specific sites.

\section{California--Continued}

Elmira, Galt, Graton, Lucerne, Meridian, West Sacramento.

12 September (P) Southern Càlifornia

origin time: $\quad 115755.4$

Epicenter: $\quad 34.38 \mathrm{~N}, 119.77 \mathrm{~W}$.

Depth :

Magnitude:

$6 \mathrm{~km}$

$3.6 \mathrm{ML}$

Intensity $V$ : Santa Barbara (few windows cracked; light furniture and small objects shifted; hairline cracks in exterior walls; windows, doors, and dishes rattled).

Intensity IV: Goleta, Solvang, Summerland.

17 September (B) Central California

origin time: $\quad \begin{array}{lll}15 & 38 & 33.1\end{array}$

Epicenter: $\quad 36.68 \mathrm{~N} .121 .36 \mathrm{~W}$.

Depth:

Magnitude:

$6 \mathrm{~km}$

Intensity IV: San Juan Bautista.

19 September (B) Northern California

Origin time: $\quad \begin{array}{ll}15 & 524.5\end{array}$

Epicenter: $\quad 37.73 \mathrm{~N} .122 .56 \mathrm{~W}$. Depth : $8 \mathrm{~km}$ 
California--Continued

Intensity II: Daly City.

21 September (B) Central California

Origin time: 031857.1

Epicenter: $\quad 36.99 \mathrm{~N}, 121.68 \mathrm{~W}$.

Depth:

$8 \mathrm{~km}$

Magnitude: $\quad 2.9 \mathrm{ML}$

Intensity III: Gilroy (B), Monterey

(B), Santa Cruz (B), Watsonville

(B).

22 September (P) Southern California

Origin time: 031326.8

Epicenter: $\quad 33.87 \mathrm{~N}, 117.83 \mathrm{~W}$.

Depth:

$4 \mathrm{~km}$

Magnitude:

2.9 ML

Intensity II: Anaheim.

22 September (B) Northern California

Origin time: $\quad 032619.8$

Epicenter: $\quad 40.63 \mathrm{~N}, 123.63 \mathrm{~W}$.

Depth:

$20 \mathrm{~km}$

Magnitude: $\quad 3.7 \mathrm{mb}(\mathrm{G}), 3.7 \mathrm{ML}$

Intensity III: Rio Dell.

24 September (P) Southern California

Origin time: 020427.5

Epicenter: $\quad 34.38 \mathrm{~N}, 119.73 \mathrm{~W}$.

Depth:

Magnitude: $\quad 3.6$ ML

Intensity IV: Goleta, Isla Vista, Santa Barbara.

26 September (P) Southern California

Origin time: 053503.9

Epicenter:

Depth:

Magnitude:

$34.03 \mathrm{~N} ., 118.40 \mathrm{~W}$.

$6 \mathrm{~km}$

Intensity II: Beverly Hills (P).

4 October (P) Owens Valley area

Origin time: $\quad 164248.6$

Epicenter: $\quad 37.53 \mathrm{~N} .118 .63 \mathrm{~W}$.

Depth:

Magnitude:

$9 \mathrm{~km}$

$5.4 \mathrm{mb}(\mathrm{G}), 5.1 \mathrm{MS}(\mathrm{G})$, $5.8 \mathrm{ML}(\mathrm{B}), 5.8 \mathrm{ML}$

The press reported that in grocery stores throughout the Bishop area considerable amounts of canned and bottled goods were shaken from shelves and pictures were knocked from walls; and there was a report of a policeman being swayed back and forth and finding it difficult to stand. Boulders rolled on to roads and minor landslides occurred in the canyon areas near Bishop. Landslides were also reported near Mammoth Lakes and in Yosemite National Park. At the Union Carbide Corporation Pine Creek Mine near Bishop about 130 miners were underground at the time of the earthquake, but none were hurt. The earthquake shook rock slides loose throughout the vicinity of the mine causing some road obstruc-
California--Continued

tions. A pickup truck was jiggled over the edge on one of the high mine roads. The University of California at Berkeley reported 30 aftershocks in the following 24 hours.

Porcella (1978c) reported that eight accelerographs at Pine Flat and Buchanan Dams northeast of Fresno, California were triggered by this earthquake. The maximum recorded acceleration was less than $0.05 \mathrm{~g}$. This earthquake was felt over an area of approximately 105,000 sq km of California and Nevada (fig. 15).

All of the aftershocks listed in Table 1 were felt in the epicentral region including the town of Bishop; however, not enough specific information is available to assign intensities at individual locations for any of the aftershocks.

Intensity $\mathrm{V}_{\perp}$ :

California-

Benton (plaster cracked, small objects broken, hanging objects swung violently, hanging pictures fell, felt by all)

Bishop (Safeway supermarket just north of Bishop had a window broken and merchandise knocked from shelves. Grocery stores throughout the area reported considerable amounts of canned and bottled goods shaken from shelves, pictures knocked from walls--press report. Reports of other effects included windows broken, plaster cracked, small objects broken, and felt by al1)

Easton (cracked plaster and cracks in exterior walls, small objects overturned, felt by many)

Friant (cracked plaster, cracked interior wall, light furniture shifted, small objects and hanging pictures fell, water in small containers spilled, felt by al1)

Mammoth Lakes-Timber Ridge (a swimming pool was cracked to such an extent that it had to be drained--press report)

Paradise Camp--24 $\mathrm{km}$ northwest of Bishop (most of the bar stock and glasses were broken and stucco walls were cracked. In Paradise Estates, items were shaken from walls, a mounted deer head fell from a wall, a mirror was broken, and fluorescent light panels fell--press report). 


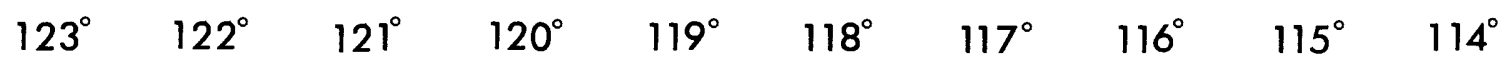

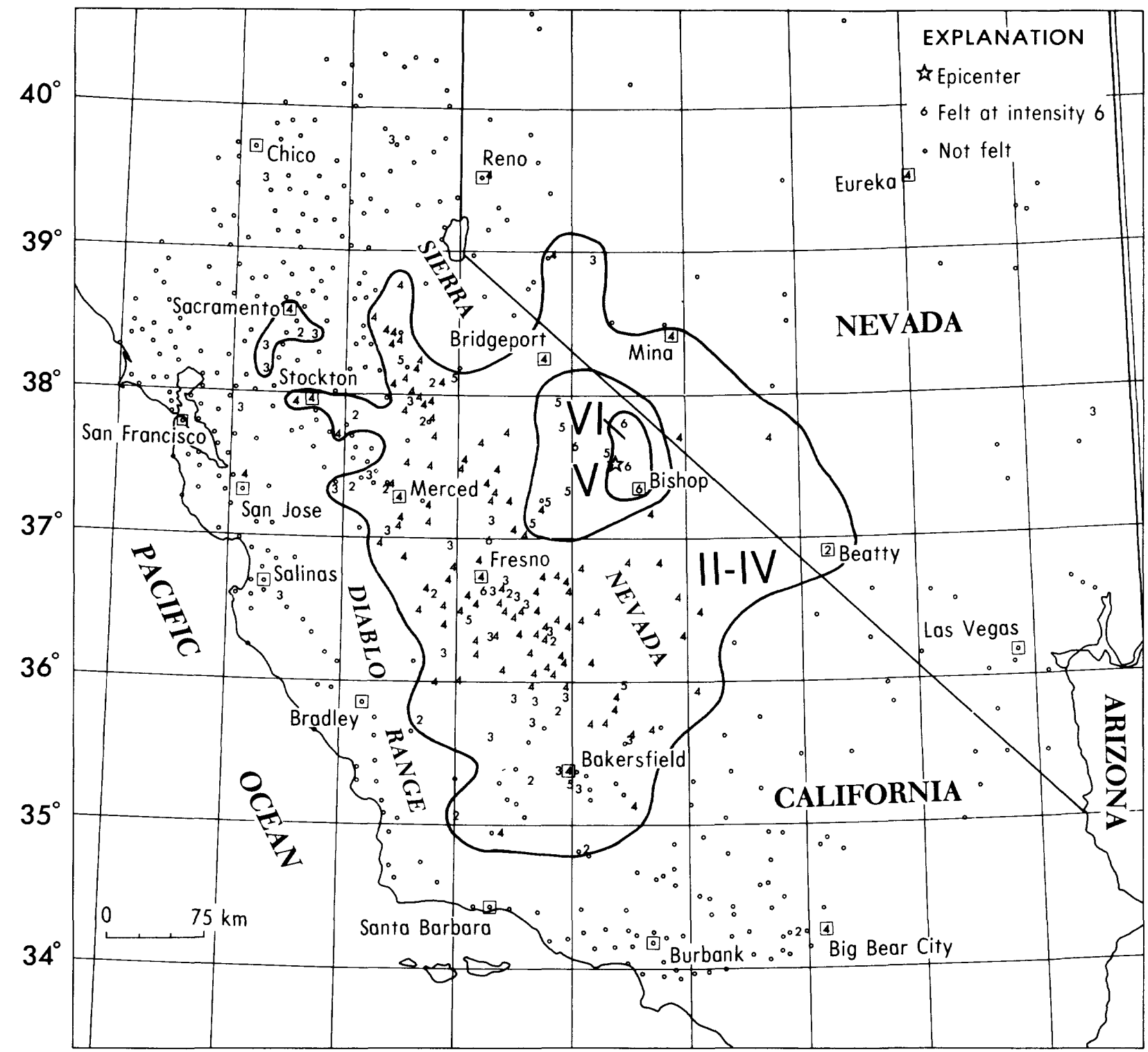

FIGURE 15.--Isoseismal map for the Owens Valley earthquake of 4 october 1978 , 1642 48.6 UTC. Roman numerals represent Modified Mercalli intensities between isoseismals; Arabic numerals are used to represent thesr intensities at specific sites.

Intensity $\mathrm{V}$ :

California--Columbia (press report), Crowley Lake, Crowley Lake Dam, Johnsondale, June Lake, Lakeshore, Lee Vining, Mono Hot Springs, Mountain Ranch, Pumpkin Center, Riverdale, Shaver Lake, Strawberry, Toms Place.
Intensity IV:

California--Ahwahnee, Altaville, Arnold, Auberry, Avenal, Badger, Bakersfield, Bass Lake, Big Bear City, Biq Creek, Big Pine, Biola, Bridgeport, Burrel, California Hot Springs, Camp Connell, Cantua Creek, Cartago, Caruthers, Castle AFB. 
California--Continued

Catheys Valley, Chinese Camp, Chowchilla, Clovis, Coarsegold, Corcoran, Coulterville, Cutler, Cuyama, Del Rey, Dos Palos, Dunlap, El Nido, El Portal, Farmersville, Farmington, Firebaugh, Fish Camp, Five Points, Fresno, Glencoe, Glennville, Goshen, Groveland, Hanford, Hathaway Pines, Helm, Holt, Hornitos, Hume, Independence, Ivanhoe, Keeler, Kerman, Kettleman City, Kingsburg, Kings Canyon, Lake Isabella, Le Grand, Lemoncove, Lemoore, Lindsay, Little Lake, London, Lone Pine, Long Barn, Mariposa, Merced, Midpines, Miramonte, Murphys, North Fork, Oakhurst, Olancha, Orange Cove, Orosi, Pacific House, Piedra, Pinedale, Pine Grove, Pioneer, Pixley, Poplar, Porterville, Posey, Prather, Rail Road Flat, Raisin, Redtop, River Pines, Sacramento, Salida, Selma, Sequoia National Park, Snelling, Soulsbyville, South Dos Palos, Springville, Squaw valley, standard, stockton, stratford, Strathmore, Tehachapi (press report), Terra Bella, Three Rivers, Tipton, Tollhouse, Tranquillity, Traver, Tulare, Tuolumne, Twain Harte, Vallecito, Visalia, Volcano, Wawona, Weldon, Wilseyville, Wishon, Woodlake, Woodville, Woody, Yosemite Lodge, Yosemite National Park.

Nevada--Dyer, Eureka, Goldfield, Mina, Sparks, Yerington.

Intensity III:

California--Alpaugh, Armona, Avery, Blairsden, Bodfish, Calwa, Chualar, Delhi, Diablo, Dinuba, Ducor, Earlimart, Fowler, Huron, Jamestown, Lamont, Laton, Liberty Farms, Lost Hills, Madera, Newman, o'Neals, Pollock Pines, Pond, Reedley, Richvale, Rio Vista, Sanger, Santa Rita Park, Wilton.

Nevada--Panaca, Schurz.

Intensity II:

California--Arcata (press report), Atwater, Cholame, Coalinga (press report), Deep Springs (press report), Elk Grove, Exeter, Green Valley Lake, Lebec, Mi-Wuk Village, Moccasin, Mono (press report), Parlier, Richgrove, San Joaquin, Stevinson, Tupman, Valley Home.

Nevada--Beatty.
Cal ifornia--Continued

5 October (B) Northern California

Origin time: 095640.4

Epicenter: $\quad 41.46 \mathrm{~N} ., 121.88 \mathrm{~W}$.

Depth:

Magnitude: $\quad 4.2 \mathrm{mb}(\mathrm{G}), 3.8 \mathrm{MS}(\mathrm{G})$, $4.1 \mathrm{ML}(\mathrm{B})$

Intensity IV: McCloud.

6 October (B) Northern California Origin time: $\quad 212634.4$ Epicenter: $\quad 40.38 \mathrm{~N}, 124.27 \mathrm{~W}$. Depth: $20 \mathrm{~km}$

Magnitude: $\quad 4.8 \mathrm{mb}(\mathrm{G}), 4.2 \mathrm{MS}(\mathrm{G})$, 4.6 ML

Felt in the coastal areas from Arcata to Cape Mendocino (B).

Intensity V: Miranda.

Intensity IV: Bayside, Bridge-

ville, Eureka, Fortuna, Rio Dell, Weott.

Intensity III: Garberville (press report), Redway (press report).

9 October (B) Central California

origin time: $\quad 204945.8$

Epicenter: $\quad 36.68 \mathrm{~N} ., 121.37 \mathrm{~W}$.

Depth:

Magnitude: $\quad 3.3 \mathrm{ML}$

Intensity III: Chualar and San Benito County (B).

10 October (B) Central California

origin time: 013836.9

Epicenter: $\quad 36.61 \mathrm{~N} .121 .28 \mathrm{~W}$.

Depth: $\quad 8 \mathrm{~km}$

Magnitude: $\quad 3.8 \mathrm{ML}$

Intensity II: San Benito County

(B).

10 October (B) California

Origin time: 042851.8

Epicenter: $\quad 38.80 \mathrm{~N}, 119.82 \mathrm{~W}$.

Depth:

Magnitude:

$10 \mathrm{~km}$

Intensity II: Markleeville area (B).

10 October (P) Central California

origin time: 090657.4

Epicenter: $\quad 35.08 \mathrm{~N}, 117.50 \mathrm{~W}$.

Depth:

Magnitude:

$6 \mathrm{~km}$

Intensity II: Edwards AFB (P).

16 October (B) Northern California

origin time: 021807.0

Epicenter: $\quad 40.46 \mathrm{~N} ., 124.30 \mathrm{~W}$.

Depth: $20 \mathrm{~km}$

Magnitude: $\quad 3.7 \mathrm{ML}(\mathrm{B})$

Intensity III: Rio Dell.

19 October (P) Southern California

origin time: 091024.5

Epicenter: $\quad 33.83 \mathrm{~N}, 118.07 \mathrm{~W}$.

Depth: $\quad 5 \mathrm{~km}$ 
Magnitude: $\quad 1.9 \mathrm{ML}$

Intensity II: Lakewood (P).

26 October (P) Owens Valley area

origin time: $\quad 190041.3$

Epicenter: $\quad 37.52 \mathrm{~N}, 118.58 \mathrm{~W}$.

Depth:

Magni tude:

$9 \mathrm{~km}$

4. $0 \mathrm{ML}(\mathrm{B})$,

$$
\text { 3.9 ML(P) }
$$

Intensity IV: Mariposa.

Intensity II : Yosemite (B).

27 October (B) Central California

origin time: $\quad 120022.2$

Epicenter: $\quad 37.26 \mathrm{~N}, 121.64 \mathrm{~W}$.

Depth:

Magnitude:

$4 \mathrm{~km}$

$3.1 \mathrm{ML}$

Intensity II: San Jose (B).

29 October (B) Northern California

Origin time: $\quad 065049.1$

Epicenter: $\quad 40.30 \mathrm{~N}, 124.73 \mathrm{~W}$.

Depth :

Magni tude:

$20 \mathrm{~km}$

Intensity IV: Rio Dell.

29 October (P) Southern California

Origin time: 080959.5

Epicenter: $\quad 33.92 \mathrm{~N} ., 118.30 \mathrm{~W}$.

Depth :

Magnitude:

$5 \mathrm{~km}$

Felt in the southwest Los Angeles Basin (P).

Intensity IV: El Segundo (press report), Hawthorne, Inglewood (press report), Los Angeles, Manhattan Beach (press report), Torrance (press report).

29 October (P) Southern California origin time: 221609.0

Epicenter: $\quad 34.32 \mathrm{~N} ., 118.63 \mathrm{~W}$.

Depth:

Magnitude:

$3 \mathrm{~km}$

Intensity IV:

Intensity II: Canoga Park, Malibu (P).

30 october (B) Central California

Origin time: $\quad 160504.6$

Epicenter: $\quad 36.67 \mathrm{~N}, 121.36 \mathrm{~W}$.

Depth :

Magnitude:

$6 \mathrm{~km}$

$3.2 \mathrm{ML}$

Intensity II: Hollister (B).

1 November (P) Imperial Valley

origin time: $\quad 064222.6$

Epicenter: $\quad 32.75 \mathrm{~N}, 115.52 \mathrm{~W}$.

Depth:

Magnitude: $\quad 2.8 \mathrm{ML}$

Intensity II: El Centro (P).

6 November (P) Owens Valley area

Origin time: $\quad 162225.0$

Epicenter: $\quad 37.53 \mathrm{~N}, 118.62 \mathrm{~W}$.

Depth:
Magnitude:

Intensity II: $\quad$ Bishop (B)

11 November (P) Southern California

origin time: $\quad 042404.6$

Epicenter: $\quad 33.63 \mathrm{~N} ., 117.88 \mathrm{~W}$.

Depth:

$3 \mathrm{~km}$

Magnitude :

$2.7 \mathrm{ML}$

Intensity $\mathrm{V}$ :

Costa Mesa (dishes, pots, and pans crashed to the floor when cupboard doors flew open--press report),

Santa Ana Heights (press reports of breakage of stored items when they were knocked loose from their "moorings").

Intensity IV: Huntington (press report), Newport Beach (press report).

12 November (B) Northern California origin time: $\quad 130757.3$

Epicenter: $\quad 39.49 \mathrm{~N} ., 122.95 \mathrm{~W}$.

Depth:

Magnitude:

$6 \mathrm{~km}$

$3.8 \mathrm{ML}$

Intensity IV: Potter Valley, Willits.

13 November (P) Southern California

origin time: $\quad 164943.1$

Epicenter: $\quad 34.43 \mathrm{~N} ., 119.70 \mathrm{~W}$.

Depth:

. $119.70 \mathrm{~W}$.

Magnitude:

$13 \mathrm{~km}$

Intensity II: Goleta, Santa Barbara.

13 November (P) Southern California

origin time: $\quad 165058.3$

Epicenter: $\quad 34.43 \mathrm{~N}, 119.70 \mathrm{~W}$.

Depth:

$15 \mathrm{~km}$

Maqnitude:

$3.3 \mathrm{ML}$

Intensity II: Goleta, Santa Barbara.

16 November (P) Southern California

origin time: $\quad 132846.7$

Epicenter: $\quad 34.15 \mathrm{~N} ., 117.60 \mathrm{~W}$.

Depth:

Magnitude:

Intensity II: Claremont (P),

Pomona (P).

19 November (P) Southern California

Origin time: $\quad 042005.6$

Epicenter: $\quad 33.85 \mathrm{~N} ., 118.17 \mathrm{~W}$.

Depth:

$12 \mathrm{~km}$

Magnitude :

$2.7 \mathrm{ML}$

Intensity II: Lakewood (P), Long Beach (P).

19 November (P) Southern California

origin time: $\quad 174056.9$

Epicenter: $\quad 33.85$ N. $118.18 \mathrm{~W}$.

Depth :

Magnitude: $\quad 3.1 \mathrm{ML}(\mathrm{P})$ 
report).

Intensity IV: Bellflower, Harbor City, Lakewood, Long Beach, Los Alamitos, Maywood.

Intensity III: Cypress, Montebello.

Intensity II: Downey (P), Hawthorne, watts (P).

19 November (P) Southern California

Origin time: $\quad 180016.4$

Epicenter: $\quad 33.85 \mathrm{~N} ., 118.17 \mathrm{~W}$.

Depth: $\quad 12 \mathrm{~km}$

Magnitude: $\quad 2.8 \mathrm{ML}(P)$

Felt in the Los Angeles area (press report).

Intensity III: North Long Beach, Watts.

20 November (P) Southern California

Origin time: $\quad 065509.4$

Epicenter: $\quad 34.15 \mathrm{~N}, 116.97 \mathrm{~W}$.

Depth:

Magnitude: $\quad 4.0 \mathrm{mb}(\mathrm{G}), 4.2 \mathrm{ML}$

Felt in Los Angeles, orange, Riverside, San Bernardino, and San Diego counties.

Intensity VI: Redlands (interior plaster walls cracked and split, hairline cracks in exterior walls, light furniture and small objects shifted, windows cracked).

Intensity $\mathrm{V}$ : Auga Caliente Springs (small objects fell). Highland (pictures knocked off walls--press report).

Intensity IV: Beaumont, Cathedral City, Fl Cajon, Hemet, Landers, Mead Valley, Morongo Valley, Palm Springs, Rancho Mirage, Rialto, Riverside, Running Springs, Sunnymead, Yucaipa.

Intensity III: Cypress, Long Beach, San Bernarảino.

Intensity II: Anaheim (P), Downey (P), Newport Beach (P), Pasadena (P), Twentynine Palms.

20 November (P) Southern California

Origin time: $\quad 065845.0$

Epicenter: $\quad 34.15 \mathrm{~N}, 116.97 \mathrm{~W}$. Depth:

$6 \mathrm{~km}$

Magnitude: $\quad 3.5 \mathrm{ML}(\mathrm{P})$

Intensity III: Cathedral City, Running Springs.

20 November (P) Southern California oriqin time: $\quad 212149.1$

Epicenter: $\quad 34.15 \mathrm{~N} ., 116.98 \mathrm{~W}$.

Depth:

$5 \mathrm{~km}$

Magnitude: $\quad 3.1 \mathrm{MI}$

Intensity II: Big Rear Lake (P).
25 November (P) Owens Valley area

origin time: 032455.3

Epicenter: $\quad 37.50 \mathrm{~N}, 118.70 \mathrm{~W}$.

Depth: $8 \mathrm{~km}$

Magnitude: $\quad 3.4 \mathrm{ML}, 3.5 \mathrm{ML}(\mathrm{B})$

Intensity II: Bishop (B).

25 November (P) Owens Valley area

Origin time: $\quad 040641.4$

Epicenter: $\quad 37.53 \mathrm{~N}, 118.68 \mathrm{~W}$.

Depth: $\quad 9 \mathrm{~km}$

Magnitude: $\quad 3.5 \mathrm{ML}, 3.3 \mathrm{ML}(\mathrm{B})$

Intensity II: Bishop (B).

1 December (P) Southern California

origin time: 232046.5

Epicenter: $\quad 33.93 \mathrm{~N}, 116.68 \mathrm{~W}$.

Depth:

Magnitude: $\quad 3.6 \mathrm{ML}$

Intensity II: Palm Springs (press report).

6 December (P) Southern California

Origin time: $\quad 100349.3$

Epicenter: $\quad 34.38 \mathrm{~N}, 119.75 \mathrm{~W}$.

Depth: $\quad 5 \mathrm{~km}$

Magnitude: $\quad 3.2 \mathrm{ML}$

Intensity III: Santa Barbara (press report).

6 December (P) Southern California

Origin time: $\quad 112148.7$

Epicenter: $\quad 34.37$ N., 119.75 W.

Depth: $\quad 5 \mathrm{~km}$

Magnitude: $\quad 2.2 \mathrm{ML}$

Intensity II: Santa Barbara (press report).

14 December (P) Southern California

Origin time: $\quad 085520.4$

Epicenter: $\quad 34.40 \mathrm{~N}, 119.50 \mathrm{~W}$.

Depth: $\quad 5 \mathrm{~km}$

Magnitude: $\quad 3.1 \mathrm{ML}$

Intensity IV: Carpenteria (press report).

Intensity III: Santa Barbara (press report).

29 December (P) Southern California

Origin time: $\quad 063030.4$

Epicenter: $\quad 33.93 \mathrm{~N}, 118.33 \mathrm{~W}$.

Depth: $\quad 3 \mathrm{~km}$

Magnitude: $\quad 2.3 \mathrm{ML}$

Intensity II: Hawthorne (P), Inglewood (P).

California--off the coast

23 May (B) Northern California

Origin time: $\quad 214202.9$

Epicenter: $\quad 40.44 \mathrm{~N} ., 124.85 \mathrm{~W}$.

Depth: $\quad 35 \mathrm{~km}$

Magnitude: $\quad 4.4 \mathrm{mb}(\mathrm{G}), 4.6 \mathrm{ML}$

Intensity IV: Bayside, Eureka, Fortuna, Miranda.

Intensity III: Bridgeville, Eel 
California--Off the coast--Continued

River and Van Duzen River Valleys (press report).

1 June (B) Northern California Origin time: $\quad 183820.9$ Epicenter: $\quad 40.34 \mathrm{~N} ., 124.84 \mathrm{~W}$. Depth: Magnitude: Intensity IV: Rio Dell.

\section{Colorado}

10 June (G) Northern Colorado

origin time: 205753.5

Epicenter: $\quad 39.78 \mathrm{~N}, 104.87 \mathrm{~W}$.

Depth:

Magnitude:

$20 \mathrm{~km}$

Intensity IV: Denver (Park Hill), Denver (1335 Rosemary St, 1680

Magnolia St, 1603 Clermont St-press report), Thornton (2501 E. 104 th St--press report), Henderson (1098 Brighton Road).

Intensity III: Commerce City. Intensity II: Thornton (post office).

\section{Delaware}

16 July (G) Southeastern Pennsylvania origin time: $\quad 063937.8$

See Pennsylvania listing.

\section{Florida}

12 January Central florida

origin time: 2110

Epicenter: Not located.

Depth:

Magnitude:

Intensity IV: Haines City (press

report).

6 November Northern Florida

origin time: 2300

Epicenter: $\quad 30.20 \mathrm{~N} ., 82.65 \mathrm{~W}$.

Depth:

Magnitude:

None computed.

None computed.

The epicenter given is a macroseismic location taken from the felt information. The magnitude is estimated to be less than 2.0 . There were reports of two events, on November 14 at 2014 UTC and on November 16 at 1900 UTC; both were reported felt, but neither were recorded on the nearest seismographs and may not have been seismic.
Florida--Continued

Intensity IV: Lake City (press reported doors rattled and houses shook).

Hawaii

The locations 1 isted below that are followed by $(\mathrm{H})$ designate intensity values assigned by the Hawaiian Volcano Observatory.

4 January (H) Island of Hawaii

origin time: $\quad 184227.2$

Epicenter: $\quad 19.33 \mathrm{~N}, 155.18 \mathrm{~W}$.

Depth:

Magnitude:

$$
10 \mathrm{~km}
$$

$3.7 \mathrm{ML}$

$I_{i}$ Eensity IV: $\mathrm{HilO}(\mathrm{H})$.

Intensity III: Kurtistown (H), Isaupahoehoe $(\mathrm{H})$, Volcano $(\mathrm{H})$.

5 January (H) Island of Hawaii

origin time: 135846.6

Epicenter: $\quad 19.32 \mathrm{~N} ., 155.27 \mathrm{~W}$.

Depth:

$10 \mathrm{~km}$

Magnitude: $\quad 3.2 \mathrm{ML}$

Intensity IV: Ainahou Ranch (H).

11 January (H) Island of Hawaii

origin time: 083311.3

Epicenter: $\quad 19.33 \mathrm{~N}, 155.22 \mathrm{~W}$.

Depth:

Magnitude:

$10 \mathrm{~km}$

Intensity IV: Hilo (H).

Intensity III: Glenwood $(\mathrm{H})$, Hamakua (H), Mountain View (H), Puna (H), Volcano (H).

13 January (H) Island of Hawaii

origin time: $\quad 003406.1$

Epicenter: $\quad 19.53 \mathrm{~N}, 155.95 \mathrm{~W}$.

Depth:

Intensity III: Kona (H).

23 January (H) Island of Hawaii

origin time: $\quad 062138.5$

Epicenter: $\quad 19.33 \mathrm{~N} ., 155.06 \mathrm{~W}$.

Depth:

Magnitude:

$9 \mathrm{~km}$

3.7 ML

Intensity IV: Glenwood (H), Hilo

(H), Kalapana (H), Kurtistown (H), Mountain View (H), Volcano (H). Intensity III: Papaikou (H).

23 January (H) Island of Hawaii

origin time: $\quad 062826.9$

Epicenter: $\quad 19.34 \mathrm{~N} ., 155.07 \mathrm{~W}$.

Depth:

Magnitude :

$9 \mathrm{~km}$

$3.6 \mathrm{ML}$

Intensity IV: Glenwood (H), Hilo

(H), Kurtistown (H), Mountain View

(H), Volcano (H).

Intensity III: Papaikou (H). 
25 January (H) Island of Hawaii

Origin time: 011816.8

Epicenter: $\quad 19.33 \mathrm{~N}, 155.10 \mathrm{~W}$.

Depth:

$9 \mathrm{~km}$

Magnitude: $\quad 3.2 \mathrm{ML}$

Intensity III: Hilo (H).

3 February (H) Island of Hawaii

Origin time: $\quad 053245.8$

Epicenter: $\quad 19.43 \mathrm{~N} ., 155.30 \mathrm{~W}$.

Depth:

Magnitude: $\quad 3.5 \mathrm{ML}$

Intensity IV: Glenwood (H), Hilo

(H), Kurtistown (H), Mountain View

(H), Volcano (H).

Intensity III: Honomu (H), Papaikou (H).

14 February (H) Island of Hawaii

origin time: 200506.2

Epicenter: $\quad 19.40 \mathrm{~N} ., 155.28 \mathrm{~W}$.

Depth:

$15 \mathrm{~km}$

Magnitude: $\quad 3.4 \mathrm{ML}$

Intensity III: Hawailan Volcano

Observatory (H), Volcano (H).

25 February (H) Island of Hawaii

origin time: 085527.2

Epicenter: $\quad 19.44 \mathrm{~N} ., 156.32 \mathrm{~W}$.

Depth:

$17 \mathrm{~km}$

Magnitude:

$3.2 \mathrm{ML}$

Intensity III: Holualoa.

2 March (H) Island of Hawaii

Origin time: $\quad \begin{array}{lll}10 & 51 & 02.9\end{array}$

Epicenter: $\quad 19.33 \mathrm{~N}, 155.22 \mathrm{~W}$.

Depth:

Magnitude: $\quad 3.7 \mathrm{M}$

Intensity IV: Hilo (H), Volcano (H).

21 March (H) Island of Hawaii

origin time: 051325.4

Epicenter: $\quad 19.37 \mathrm{~N}, 155.42 \mathrm{~W}$.

Depth:

$12 \mathrm{~km}$

Magnitude: $\quad 3.3 \mathrm{ML}$

Intensity III: Kukaiau Ranch.

3 April (H) Island of Hawai i

origin time: $\quad 042832.9$

Epicenter: $\quad 19.30 \mathrm{~N}, 155.19 \mathrm{~W}$.

Depth:

Magnitude: $\quad 3.0 \mathrm{MI}$

Intensity III: Volcano (H).

6 April (H) Island of Hawaii

origin time: $\quad 134714.1$

Epicenter: $\quad 19.33 \mathrm{~N}, 155.13 \mathrm{~W}$.

Depth:

Magnitude:

$10 \mathrm{~km}$

3.2 ML

Intensity III: Hilo (H).

8 April (H) Island of Hawaii

origin time: 024344.2

Epicenter: $\quad 19.83$ N., 155.14 W.

Depth:

Magnitude:

$29 \mathrm{~km}$

$3.6 \mathrm{ML}$

Intensity IV: Hilo (H), Papaiko
(H), Pepeekeo (H), Honolii (H).

Intensity III: Glenwood (H), Hawaiian Volcano Observatory (H), Honokaa (H), Laupahoehoe (H).

Intensity II: Ahualoa (H), Hawaiian Ocean View Estates (H), Waimea (H).

13 April (H) Island of Hawaii

Origin time: $\quad 065512.7$

Epicenter: $\quad 19.34 \mathrm{~N}, 155.07 \mathrm{~W}$.

Depth:

Magnitude: $\quad 3.4 \mathrm{ML}$

Intensity IV: Hawaii Volcanoes

National Park (H), Hilo (H).

3 May (H) Island of Hawaii

Origin time: $\quad 172906.4$

Epicenter: $\quad 19.29$ N., $155.50 \mathrm{~W}$.

Depth :

Magnitude:

$8 \mathrm{~km}$

Intensity IV: Pahala (H).

23 May (H) Island of Hawaii

Origin time: 070926.7

Epicenter: $\quad 19.31 \mathrm{~N}, 155.22 \mathrm{~W}$.

Depth :

Magnitude:

$10 \mathrm{~km}$

Intensity IV: Hilo $(\mathrm{H})$.

Intensity III: Glenwood ( $\mathrm{H}$ ), Hawaii

Volcanoes National Park (H), Moun-

tainview (H), Papaikou (H), Volcano (H).

3 June (H) Island of Hawaii

Origin time: 010202.1

Epicenter: $\quad 19.67 \mathrm{~N}, 155.16 \mathrm{~W}$.

Depth :

$14 \mathrm{~km}$

Magnitude:

$3.2 \mathrm{ML}$

Intensity IV: $\mathrm{HilO}(\mathrm{H})$

Intensity III: Glenwood ( $\mathrm{H}$ ), Hawaii

Volcanoes National Park (H),

Honokaa (H), Kalapana (H), Paauilo

(H), Volcano (H).

4 June (H) Island of Hawai i

Origin time: 080044.9

Epicenter: $\quad 19.81 \mathrm{~N}, 155.79 \mathrm{~W}$.

Depth :

Magnitude:

$16 \mathrm{~km}$

Intensity IV: Kona (H).

12 June (H) Island of Hawaii

origin time: 215712.1

Epicenter: $\quad 19.40 \mathrm{~N}, 155.26 \mathrm{~W}$.

Depth: $\quad 3 \mathrm{~km}$

Magnitude: $\quad 3.0 \mathrm{ML}$

Intensity IV: Kilauea Caldera (H).

Intensity III : Hawaiian Volcano

Observatory (H), Hawaii Volcanoes

National Park (H), Volcano (H).

13 June (H) Island of Hawaii

origin time: 024935.0

Epicenter: $\quad 18.90 \mathrm{~N} ., 155.46 \mathrm{~W}$.

Depth:

Magnitude:

$15 \mathrm{~km}$

Intensity IV: Hawaiian Ocean View Estates $(\mathrm{H})$, Naalehu (H). 
Hawaii--Continued

13 June (H) Island of Hawaii

Origin time: $\quad 165401.2$

Epicenter: $\quad 19.34 \mathrm{~N} ., 155.05 \mathrm{~W}$.

Depth: $\quad 6 \mathrm{~km}$

Magnitude: $\quad 3.1 \mathrm{ML}$

Intensity IV: Kalapana (H), Kapaahu (H).

Intensity III: Mountainview (H), Volcano $(\mathrm{H})$, Wainaku $(\mathrm{H})$.

21 June (H) Island of Hawai

Origin time: $\quad 102044.1$

Epicenter: $\quad 19.32 \mathrm{~N}, 155.22 \mathrm{~W}$.

Depth: $\quad 10 \mathrm{~km}$

Magnitude: $\quad 3.4 \mathrm{ML}$

Intensity III: Hawaii Volcanoes

National Park (H), Pahala (H), Volcano (H).

23 June (H) Island of Hawaii

origin time: $\quad 114758.6$

Epicenter: $\quad 19.32 \mathrm{~N} .155 .26 \mathrm{~W}$.

Depth:

Magnitude:

$11 \mathrm{~km}$

$4.9 \mathrm{mb}(\mathrm{G}), 4.2 \mathrm{ML}$

Intensity IV: Captain Cook, Hawaii

Volcanoes National Park, Hamakua

(H), Hilo, Honokaa, Honomu, Keaau, Kukuihaele, Kurtistnwn, Naalehu, Paauhau, Paauilo, Fahala, Pahoa, Papaikou, Pepeekeo, Puna (H), Volcano.

Intensity III: Kau, Kohala, Kona. Intensity II: Kalaupapa.

30 June (H) Island of Hawaii

Origin time: $\quad 222802.8$

Epicenter: $\quad 19.17$ N., $155.48 \mathrm{~W}$.

Depth:

Magnitude:

$9 \mathrm{~km}$

$3.1 \mathrm{ML}$

Intensity III: Hawaiian Ocean View

Estates $(\mathrm{H})$.

1 July (H) Island of Hawai i

Origin time: $\quad 191813.3$

Epicenter: $\quad 19.32 \mathrm{~N}, 155.12 \mathrm{~W}$.

Depth:

Magnitude:

$7 \mathrm{~km}$

$3.9 \mathrm{ML}$

Intensity IV: Hilo (H), Puna (H).

Intensity III: Mauna Loa Observatory

(H), Pohakuloa (H).

Intensity II: $\operatorname{Rohala}(\mathrm{H}), \operatorname{Kona}(\mathrm{H})$.

4 July (H) Island of Hawaii

Origin time: 070516.3

Epicenter: $\quad 19.35$ N., $155.08 \mathrm{~W}$.

Depth :

Magnitude:

$9 \mathrm{~km}$

$3.0 \mathrm{ML}$

Intensity III: Glenwood (H).

9 July (H) Island of Hawaii

Origin time: $\quad 230756.9$

Epicenter: $\quad 19.37 \mathrm{~N} ., 155.10 \mathrm{~W}$.

Depth:

Magnitude: $\quad 3.1 \mathrm{ML}$

Intensity III: Hilo (H).

$14 \mathrm{July}$ (H) Island of Hawai origin time: $\quad 125637.2$
Hawai--Continued

Epicenter:

Depth:

Magnitude :

$19.35 \mathrm{~N} ., 155.25 \mathrm{~W}$.

Intensity III: Volcano (H).

$10 \mathrm{~km}$

$3.2 \mathrm{ML}$

16 July (H) Island of Hawaii

Origin time: $\quad 182941.8$

Epicenter: $\quad 19.35 \mathrm{~N} ., 155.02 \mathrm{~W}$.

Depth:

Magnitude:

$8 \mathrm{~km}$

Kalapana (H).

Intensity III: Glenwood $(\mathrm{H}), \mathrm{HilO}$ (H).

9 August ( $\mathrm{H}$ ) Island of Hawai

Origin time: $\quad 071010.3$

Epicenter: $\quad 19.30 \mathrm{~N}, 155.22 \mathrm{~W}$.

Depth:

Magnitude: $\quad 3.4 \mathrm{ML}$

Intensity III: Glenwood ( $\mathrm{H}), \mathrm{HilO}$

(H), Kurtistown (H), Mountainview

(H), Volcano (H).

12 August (H) Island of Hawaii

origin time: $\quad 105250.5$

Epicenter: $\quad 19.33 \mathrm{~N}, 155.11 \mathrm{~W}$.

Depth:

Magnitude:

$10 \mathrm{~km}$

Intensity IV:

$3.1 \mathrm{ML}$

Hilo (H).

Intensity III : Mountainview (H),

Volcano $(\mathrm{H})$.

29 August (H) Island of Hawaii

Origin time: 214408.9

Epicenter: $\quad 19.32 \mathrm{~N} ., 155.20 \mathrm{~W}$.

Depth:

Magnitude: $\quad 3.1 \mathrm{ML}$

Intensity II: Hilo (press report).

30 August (H) Island of Hawaii

origin time: $\quad 224015.7$

Epicenter: $\quad 19.34 \mathrm{~N} .155 .03 \mathrm{~W}$.

Depth :

Magnitude: $\quad 3.1 \mathrm{ML}$

Intensity III: Rlacksand Subdivision

(H), Hilo (H), Kalapana (H), Volcano (H).

31 August (H) Island of Hawaii

Origin time: 230721.4

Epicenter: $\quad 19.01 \mathrm{~N} ., 155.48 \mathrm{~W}$.

Depth:

Magnitude:

$35 \mathrm{~km}$

Intensity IV: $\mathrm{Kau}(\mathrm{H})$

Intensity III : Ahualoa (H), Ainaloa

(H), Glewnood (H), Hawaiian Beaches (H), Hawailan Paradise Park (H), Hawailian Volcano Observatory (H), Hilo (H), Honokaa (H), Kamuela (H), Volcano Golf Course (H).

3 September (H) Island of Hawai

origin time: $\quad 142610.5$

Epicenter: $\quad 19.32 \mathrm{~N}, 155.20 \mathrm{~W}$.

Depth: $\quad 9 \mathrm{~km}$

Magnitude: $\quad 3.0 \mathrm{ML}$

Intensity III: Volcano (H). 
5 September (H) Island of Hawaii

Origin time: $\quad 202646.8$

Epicenter: $\quad 19.33 \mathrm{~N} ., 155.23 \mathrm{~W}$.

Depth:

$10 \mathrm{~km}$

Magnitude: $\quad 3.7 \mathrm{ML}$

Intensity IV: Hilo (H).

Intensity III: Glenwood $(\mathrm{H}), \mathrm{H}$ awaii

Volcanoes National Park (H),

Hawaiian Volcano Observatory (H),

Volcano $(\mathrm{H})$.

12 September (H) Island of Hawaii

origin time: 061606.1

Epicenter: $\quad 19.33 \mathrm{~N} ., 155.11 \mathrm{~W}$.

Depth:

$10 \mathrm{~km}$

Magnitude: $\quad 4.1 \mathrm{ML}$

Intensity IV: Glenwood, Hawaii Volcanoes National Park, Hilo, Honomu, Keaau, Kurtistown, Mountainview (H), Ookala, Pahoa (H), Papaaloa, Volcano.

Intensity III: Hakalau (H), Kalapana (H), Naanaleau (H), Papaikou. Intensity II: Honokaa.

19 September (H) Island of Hawaii

origin time: $\quad 094429.2$

Epicenter: $\quad 19.39 \mathrm{~N}, 155.28 \mathrm{~W}$.

Depth :

$4 \mathrm{~km}$

Magnitude: $\quad 3.2 \mathrm{ML}$

Intensity III: Namakanipaio (H), Volcano $(\mathrm{H})$.

20 September (H) Island of Hawaii

Origin time: 014108.7

Epicenter: $\quad 19.44 \mathrm{~N}, 155.40 \mathrm{~W}$.

Depth:

$9 \mathrm{~km}$

Magnitude: $\quad 3.3 \mathrm{ML}$

Intensity IV: Red Hill (H).

20 September (H) Island of Hawai

origin time: $\quad 232027.0$

Epicenter: $\quad 19.52 \mathrm{~N}, 155.88 \mathrm{~W}$.

Depth:

$9 \mathrm{~km}$

Maqnitude: $\quad 3.1 \mathrm{ML}$

Intensity IV: Kealakekua $(H)$.

30 September (H) Island of Hawaii

origin time: 235613.3

Epicenter: $\quad 19.38 \mathrm{~N} ., 155.45 \mathrm{~W}$.

Depth:

$9 \mathrm{~km}$

Magnitude:

$3.2 \mathrm{ML}$

Intensity IV: Pahala (H).

Intensity III: Glenwood $(\mathrm{H})$, Naalehu $(\mathrm{H})$.

Intensity II: $\mathrm{HilO}(\mathrm{H})$.

15 October (H) Island of Hawai i

Origin time: $\quad 153548.6$

Epicenter: $\quad 19.38 \mathrm{~N}, 155.07 \mathrm{~W}$.

Depth:

$9 \mathrm{~km}$

Magnitude: $\quad 3.3 \mathrm{ML}$

Intensity IV: Hawaiian Paradise

Park (H), Kalapana (H).

Intensity III: Hilo (H), Kaimu (H).

Intensity II: Volcano $(\mathrm{H})$, Hawaii

Volcanoes National Park (H).

28 October (H) Island of Hawaii

origin time: 223733.2

Epicenter: $\quad 21.56 \mathrm{~N} ., 157.98 \mathrm{~W}$.

Depth :

Magnitude:

$5 \mathrm{~km}$

Honolulu, Kaaawa

Kapalama, Kunia, Pearl City,

Waipahu.

Intensity III: Kaneohe $(\mathrm{H})$.

Intensity II: Waikiki.

22 November (H) Island of Hawaii

origin time: $\quad 232314.8$

Epicenter: $\quad 19.35 \mathrm{~N} ., 155.05 \mathrm{~W}$.

Depth:

Magnitude: $\quad 3.3 \mathrm{MI}$

Intensity III: Wahaula Visitors

Center $(\mathrm{H})$.

Intensity II: Blacksand Subdivision (H), Hawaii Volcanoes National Park

(H).

23 November (H) Island of Hawaii

origin time: 131615.9

Epicenter: $\quad 19.23 \mathrm{~N}, 155.55 \mathrm{~W}$.

Depth:

Magnitude: $\quad 4.2 \mathrm{ML}$

Intensity IV: Hawaiian Ocean View

Estates $(\mathrm{H})$, Naalehu $(\mathrm{H})$, Pahala (H).

Intensity III: Honaunau $(\mathrm{H})$, Keokea ( $\mathrm{H}$ ).

Intensity II: Ahualo (H), Hilo (H), Kailua-Kona (H), Kainaliu (H),

Keauhou (H), Mauna Loa Observatory

(H), Papaikou (H).

28 November (H) Island of Hawaii

origin time: $\quad 113136.7$

Epicenter: $\quad 19.31 \mathrm{~N}, 155.22 \mathrm{~W}$.

Depth:

Intensity III: Ainahou Ranch (H).

Intensity II: Volcano (H).

29 November (H) Island of Hawaii

origin time: $\quad 075040.3$

Fpicenter: $\quad 19.65$ N., $156.01 \mathrm{~W}$.

Depth:

Magnitude: $\quad 3.3 \mathrm{ML}$

Intensity III: Kona $(\mathrm{H})$.

29 November (H) Island of Hawaii

origin time: $\quad 084259.6$

Epicenter: $\quad 19.37 \mathrm{~N}, 155.45 \mathrm{~W}$.

Depth:

Maqnitude:

Intensity III: Kailua-Kona (H),

Pahala (H).

Intensity II: Volcano Golf Course

(H).

1 December (H) Island of Hawaii

origin time: $\quad 012239.4$

Epicenter: $\quad 19.35$ N., $155.28 \mathrm{~W}$.

Depth :

$29 \mathrm{~km}$

Magnitude: $\quad 3.4 \mathrm{MI}$

Intensity III: Ainaloa $(H)$. 
5 December ( $H$ ) Island of Hawaii

Origin time: $\quad 213639.7$

Epicenter: $\quad 19.33 \mathrm{~N}, 155.19 \mathrm{~W}$.

Depth:

$10 \mathrm{~km}$

Magnitude: $\quad 3.8 \mathrm{ML}$

Intensity III: Ainaioa (H), Hilo

(H), Kalapana (H), Nanawale (H), Volcano (H).

13 December (H) Island of Hawaii

Origin time: $\quad 044431.5$

Epicenter: $\quad 19.36 \mathrm{~N}, 155.08 \mathrm{~W}$.

Depth :

Magnitude: $\quad 3.6 \mathrm{ML}$

Intensity IV: Glenwood $(\mathrm{H})$, Kalapana (H), Mountain View $(H)$, Pahoa (H).

Intensity III: Hilo (H).

14 December (H) Island of Hawaii

Origin time: $\quad 141244.9$

Epicenter: $\quad 19.32 \mathrm{~N}, 155.22 \mathrm{~W}$.

Depth :

$10 \mathrm{~km}$

Magnitude: $\quad 4.1 \mathrm{ML}$

Intensity IV: Glenwood (H), Hilo

(H), Kurtistown (H), Mountain View (H).

Intensity III: Ainaloa (H), Volcano (H).

Intensity II : Blacksand Subdivision (H), Hawaii Volcanoes National Park (H).

27 December (H) Island of Hawaii

origin time: $\quad 104055.7$

Epicenter: $\quad 19.33 \mathrm{~N}, 155.22 \mathrm{~W}$.

Depth:

$10 \mathrm{~km}$

Magnitude: $\quad 4.0 \mathrm{ML}$

Intensity IV: Ainaloa (H), Glenwood (H), Hilo (H), Kurtistown (H), Mountain View (H), Papaikou (H), Volcano ( $\mathrm{H})$.

Intensity III: Ahualoa (H), Hawaii Volcanoes National Park (H), Hawaiian Beaches ( $H$ ), Hawaiian Ocean View Estates (H), Honokaa (H), $\operatorname{Kau}(\mathrm{H}), \operatorname{Kona}(\mathrm{H})$.

Idaho

3 April (G) Western Idaho

origin time:

$\begin{array}{lll}10 & 10 & 08.1\end{array}$

Epicenter:

$44.05 \mathrm{~N},, 116.36 \mathrm{~W}$.

Depth :

$5 \mathrm{~km}$

Magnitude:

$3.2 \mathrm{ML}, 3.6 \mathrm{ML}(\mathrm{D})$

Intensity IV: Sweet.

20 April (G) Southeastern Idaho

origin time: $\quad 145647.6$

Epicenter: $\quad 42.66$ N., $111.55 \mathrm{~W}$.

Depth:

$5 \mathrm{~km}$

Magnitude:

$2.5 \mathrm{ML}$

Intensity IV: Conda.

Intensity III: Soda springs (telephone report).

23 April (G) Western Montana

origin time: 232437.0

See Montana listing.

29 July (U) Northern Utah

origin time: $\quad 140403.2$

See Utah listing.

28 September (G) Southern Idaho

Origin time: $\quad 085820.7$

Epicenter: $\quad 42.10 \mathrm{~N} ., 112.33 \mathrm{~W}$.

Depth: $5 \mathrm{~km}$

Magnitude: $\quad 2.7 \mathrm{ML}$

Intensity IV: Malad City.

24 October (U) Southeastern Idaho

origin time: $\quad 203059.3$

Epicenter: $\quad 42.55 \mathrm{~N}, 111.84 \mathrm{~W}$.

Depth:

Magnitude: $\quad 4.2 \mathrm{mb}(\mathrm{G}), 4.3 \mathrm{ML}(\mathrm{G})$,

Intensity VI: $4.1 \mathrm{ML}$

Idaho--Thatcher (cracked plaster, cracked cement foundation).

Intensity $\mathrm{V}$ :

Idaho--Lava Hot Springs (furniture shifted; windows, doors, and dishes rattled; felt by many).

Intensity IV:

Idaho--Aberdeen, Arimo, Bancroft, Downey, Grace, Inkom, McCammon, Pocatello, Swanlake.

Intensity II:

Idaho--Franklin.

Utah--Plymouth.

29 October (G) Central Idaho

origin time: $\quad 134644.5$

Epicenter: $\quad 44.96$ N., $114.27 \mathrm{~W}$.

Depth: $5 \mathrm{~km}$

Magnitude: $\quad 4.2 \mathrm{mb}, 5.0 \mathrm{ML}$, $5.0 \mathrm{ML}(\mathrm{D})$

This earthquake was felt over an area of approximately $25,000 \mathrm{sq} \mathrm{km}$ of Idaho and Montana (fig. 16).

Intensity $\mathrm{V}$ :

Idaho--

Ellis (light furniture and small objects shifted, hanging pictures knocked out of place, felt by all).

Lowman (light furniture shifted; small objects fell; windows, doors, and dishes rattled; awakened and felt by many).

Intensity IV:

Idaho--Carmen, Challis, Clayton, Cobalt, Hailey, Lemhi, Northfork, Salmon, Shoup, Sweet, Tendoy.

Intensity III:

Idaho--Golden.

Montana--Jackson.

Intensity II:

Idaho--Garden Valley, Leadore. 


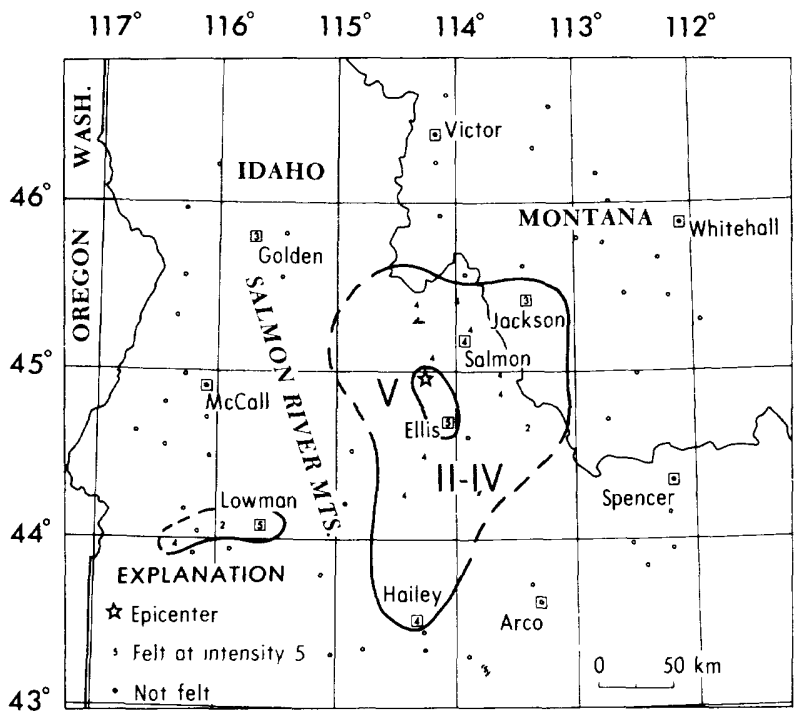

FIGURE 16.--Isoseismal map for the central Idaho earthquake of 29 October 1978, 134644.5 UTC. Roman numerals represent Modified Mercalli intensities between isoseismals; Arabic numerals are used to represent these intensities at specific sites.

Idaho--Continued

20 November (G) Central Idaho

Origin time: $\quad 142551.8$

Epicenter: $\quad 44.00 \mathrm{~N},, 114.41 \mathrm{~W}$.

Depth:

$5 \mathrm{~km}$

Magnitude: $\quad 3.2 \mathrm{ML}, 3.7 \mathrm{ML}(\mathrm{D})$

Intensity IV: Clayton.

30 November (U) Southeastern Idaho

origin time: 065340.1

Epicenter: $\quad 42.11 \mathrm{~N} ., 112.49 \mathrm{~W}$.

Depth :

$4 \mathrm{~km}$

Magnitude: $\quad 4.6 \mathrm{mb}(\mathrm{G}), 4.7 \mathrm{ML}$

This earthquake is located in the Pocatello Valley of Idaho in the same area as the March 27, 1975 intensity VIII event of magnitudes $6.1 \mathrm{mb}(\mathrm{G}), 6.0 \mathrm{MS}(\mathrm{G})$, and 6.1 $M L(G)$. It was felt over an area of approximately $18,000 \mathrm{sq} \mathrm{km}$ of Idaho and Utah (fig. 17).

Intensity $\mathrm{V}$ :

Utah--Snowville (water in small containers spilled; small objects shifted; windows, doors, and dishes rattled; felt by all). Intensity IV:

Idaho--Arimo, Clifton, Dayton, Elba, Franklin, Holbrook, Malad City, Malta, Naf, Pocatello, Stone, Swanlake, Weston.

Utah--Rear River City, Cache Junction, Clarkston, Corinne, Cor-
Idaho--Continued

nish, Fielding, Garland (press report), Honeyville, Howell, Huntsville, Lewiston, Newton, Plymouth, Providence, Richmond, Riverside, Tremonton.

Intensity III:

Utah--Kelton.

Intensity II:

Idaho--American Falls, Inkom.

Utah--Wellsville.

30 November (U) Southeastern Idaho

Origin time: $\quad 115509.3$

Epicenter: $\quad 42.11 \mathrm{~N} ., 112.55 \mathrm{~W}$.

Depth: $\quad 4 \mathrm{~km}$

Magnitude: $\quad 3.5 \mathrm{ML}$

Intensity II: Stone.

20 December (U) Southeastern Idaho

origin time: $\quad 134622.6$

Epicenter: $\quad 42.12 \mathrm{~N} ., 112.49 \mathrm{~W}$.

Depth :

Magnitude: $\quad 3.9 \mathrm{ML}$

Intensity IV:

Idaho--Holbrook, Malad City, stone, Swanlake.

Utah--Clarkston, Lewiston, North Ogden, Portage, Riverside, Snowville.

Intensity III:

Utah--Garland.

Intensity II:

Idaho--Inkom.

Utah--Fielding, Trenton.

Illinois

2 June (S) Southern Illinois

Origin time: $\quad 020728.8$

Epicenter: $\quad 38.42 \mathrm{~N} ., 88.46 \mathrm{~W}$.

Depth:

Magnitude:

$20 \mathrm{~km}$

$3.5 \mathrm{mbLg}(\mathrm{V})$

Intensity IV: Fairfield, Flora.

29 August (S) Southern III inois

Origin time: 070550.3

Epicenter: $\quad 38.53 \mathrm{~N} ., 88.22 \mathrm{~W}$.

Depth: $17 \mathrm{~km}$

Magnitude: $\quad 2.4 \mathrm{mbLg}$

Intensity II: West salem.

20 September ( $S$ ) Eastern Missouri origin time: 122408.8

See Missouri Iisting.

5 December (S) Southern Illinois

Origin time: 014801.3

Epicenter: $\quad 38.62 \mathrm{~N} ., 88.36 \mathrm{~W}$.

Depth:

Magnitude: $\quad 3.5 \mathrm{mbLg}$

This earthquake was felt over an area of approximately $11,5000 \mathrm{sq} \mathrm{km}$ of Illinois and Indiana (fig. 18). 


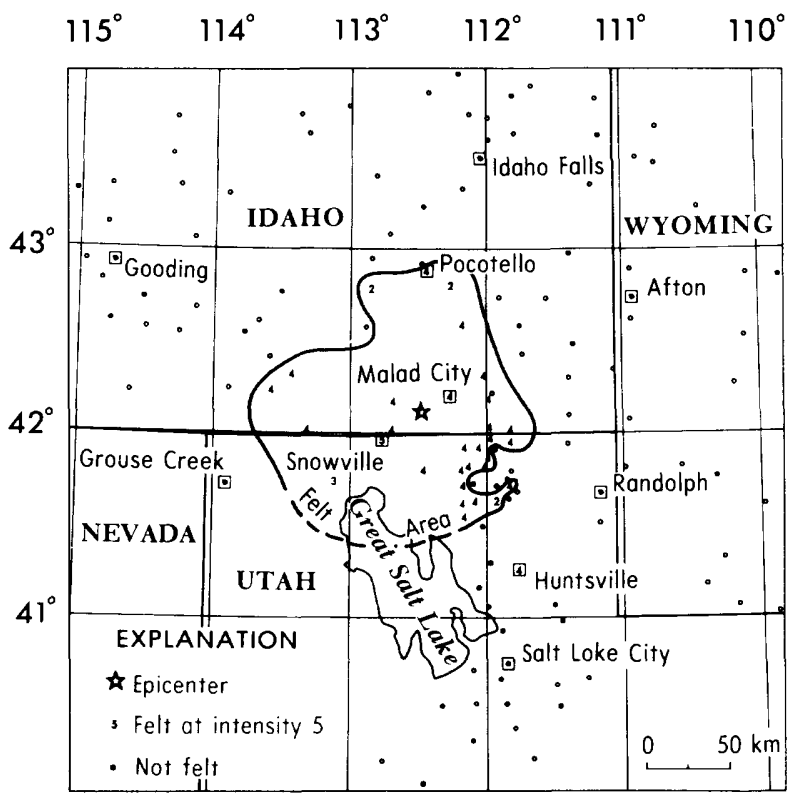

FIGURE 17.--Isoseismal map for the southeastern Idaho earthquake of 30 November 1978, 0653 40.1 UTC. Roman numerals represent Modified Mercalli intensities between isoseismals; Arabic numerals are used to represent these intensities at specific sites.

Illinois--Continued

Intensity $\mathrm{V}$ :

Illinois--

Barnhill (one report of cracked plaster; hanging pictures swung; buildings creaked and trembled; windows, doors, and dishes rattled; felt by many)

Newton (liaht furniture and small objects shifted; hanging pictures swung; windows, doors, and dishes rattled; felt by al1)

West Liberty (light furniture shifted; small objects fell; windows, doors, and dishes rattled; felt by many)

Willow Hill (few windows cracked; small objects shifted; hanging pictures swung; windows, doors, and dishes rattled; felt by many).

Intensity IV:

Illinois-Alma, Annapolis, Belle

Rive, Bluford, Bone Gap,

Bridgeport, Browns, Carmi, Cisne,

Coulterville, Dieterich, Dundas,

Edgewood, Effingham, Fairfield,

Farina, Flora, Geff, Golden Gate, Grayville, Hoyleton, Ingraham, Iola, Iuka, Johnsonville, Kell, Kinmundy, Lancaster, Louisville,
Illinois--Continued

Mason, Mount Erie, Noble, Olney, Opdyke, Parkersburg, Patoka, Rinard, Saint Peter, Sainte Marie, Salem, Sims, Robinson, Texico, West Union, Xenia, Yale. Indiana--Buffaloville, Owensville. Intensity III:

Illinois--Albion, Bellmont, Bonnie, Sailor Springs, Sullivan.

Intensity II:

Illinois--Bible Grove, Buckner, Fillmore, Flat Rock, Glen Carbon, Irving, Keenes, Lawrenceville, Oakdale, Shobonier, Teutopolis, West Salem, West York.

Indiana--Fairbanks, Stewartsville, Switz City.

\section{Indiana}

5 December (S) Southern Illinois origin time: 014801.3

See Illinois listing.

\section{Maine}

4 January ( J) Southwestern Maine

Origin time: $\quad 192810.8$

Epicenter: $\quad 44.04 \mathrm{~N} ., 70.51 \mathrm{~W}$.

Depth: $0 \mathrm{~km}$

Magnitude: $\quad 3.2 \mathrm{mbLg}, 3.2 \mathrm{mbLg}(\mathrm{L})$

Intensity IV: Cisco (J), Naples (press report), Otisfield (press report).

Intensity III: Harrison (J), Oxford (J), Poland (J).

Intensity II: Bridgton (J), Canton $(\mathrm{J})$, Johnson Hill (J), west Poland (J).

Maryland

16 July (G) Southeastern Pennsylvania Oriqin time: $\quad 06 \quad 3937.8$

See Pennsylvania listing.

\section{Massachusetts}

24 March (G) Southwest of Bermuda Islands Origin time: $\quad 004238.2$

Epicenter: $\quad 29.86 \mathrm{~N}, 67.39 \mathrm{~W}$.

Depth:

Magnitude:

$22 \mathrm{~km}$

$6.1 \mathrm{mb}, 6.0 \mathrm{MS}$, $6.1 \mathrm{MS}(\mathrm{B}), 6.0 \mathrm{ML}(\mathrm{P})$

Intensity IV: Massachusetts--New Bedford. Intensity III:

Massachusetts--Boston (press 


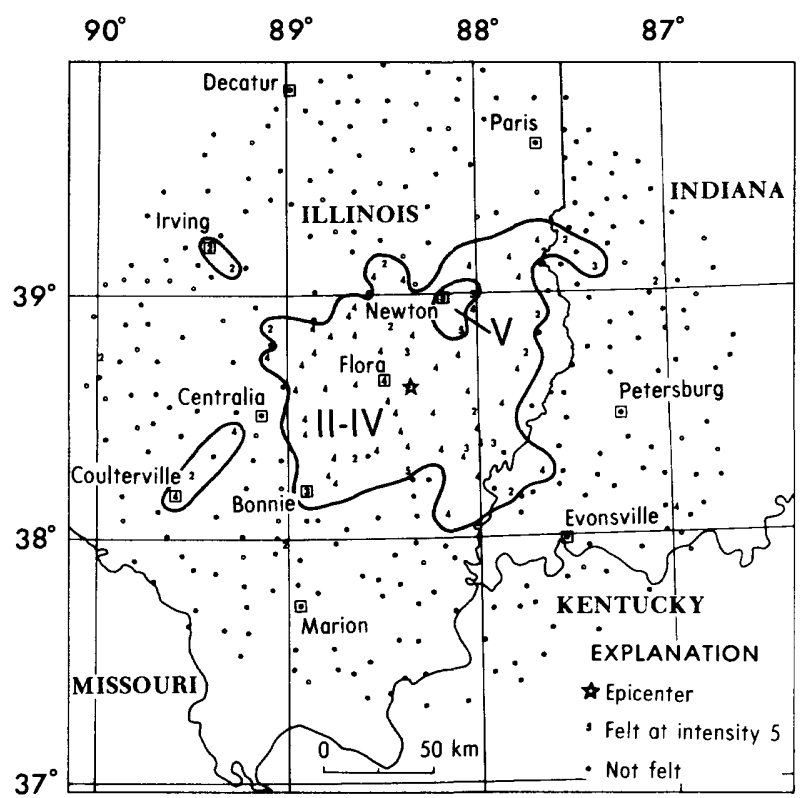

FIGURE 18.--Isoseismal map for the southern Illinois earthquake of 5 December $1978,014801.3$ UTC. Roman numerals represent Modified Mercalli intensities between isoseismals; Arabic numerals are used to represent these intensities at specific sites.

Massachusetts--Continued

report)

North Carolina--Asheville (press report), Camp Lejune (press report), Hatteras (press report), Wake County (press report).

1 September (J) Eastern Massachusetts

Origin time: 033343.6

Epicenter: $\quad 42.48 \mathrm{~N}, 71.46 \mathrm{~W}$.

Depth:

Magnitude:

$0 \mathrm{~km}$

2. $0 \mathrm{mbLg}$

Intensity III: Stow.

Intensity II: Acton.

Mississippi

11 December (G) Alabama-Mississippi border area

Origin time:

$02 \quad 06 \quad 48.2$

Epicenter:

Depth :

Magnitude:

Intensity $\mathrm{V}$ :

Alabama--

Gilbertown (light furniture shifted, small objects fell),

Melvin (small objects broken).

Intensity II:

Mississippi--Carmichael.
Missour i

31 August (S) New Madrid, Missouri region Origin time: $\begin{array}{lll}00 & 31 & 00.3\end{array}$

See Tennessee listing.

20 September (S) Eastern Missouri

Origin time: $\quad 122408.8$

Epicenter: $\quad 38.57 \mathrm{~N} ., 90.28 \mathrm{~W}$.

Depth:

$2 \mathrm{~km}$

Magnitude: $\quad 3.1 \mathrm{mbLg}, 3.0 \mathrm{mbLg}(\mathrm{G})$

O. W. Nuttli, st. Louis University, reported two places with minor damage that are not reflected in the intensity values 1 isted below. One was in southeast st. Louis where one of the concrete posts, $76 \mathrm{~cm}$ high and $25 \mathrm{~cm}$ square, which supported a wooden porch, cracked entirely across and showed evidence of slight displacement. The other occurred at Ladue where a plaster ceiling cracked down the middle.

This earthquake was felt over an area of approximately $3,700 \mathrm{sq} \mathrm{km}$ of Illinois and Missouri (fig. 19).

Intensity $\mathrm{V}$ :

Illinois--Belleville (plaster cracked in one home, small objects shifted, windows rattled), East Alton (few windows cracked; windows, doors, and dishes rattled).

Missouri--North County (few windows cracked; buildings shook; windows, doors, and dishes rattled). Intensity IV:

Illinois--Alton, Caseyville, Dupo, East Carondelet, Edwardsville, Elsah, Glen Carbon, Godfrey, Granite City, Lebanon, Litchfield (press report), Lovejoy, Madison, Marine, Moro, National Stock Yards, O'Fallon, Roxana, Smithton, South Roxana, Venice.

Missouri--Affton, Benton Park, Berkeley, Brentwood, Carondelet, Chesterfield, Christian Bechtold, Clayton, Crestwood (press report), Defiance, Ferguson, Florissant, Gravois, Jennings, Lambert Airport, Manchester, Maplewood, Northwest Plaza, Northwood, Olivette, Pierre Laclede, Portage Des Sioux, Richmond Heights, Sappington, St. Ann, St. Charles, st. Louis, st. Peters, University City, Webster Groves, West Alton.

Intensity III:

Illinois--Columbia, Wood River.

Missouri--Des Peres, Fenton.

Intensity II:

Illinois--Chicago (press report), Dow.

Missouri--Grover, Hematite. 


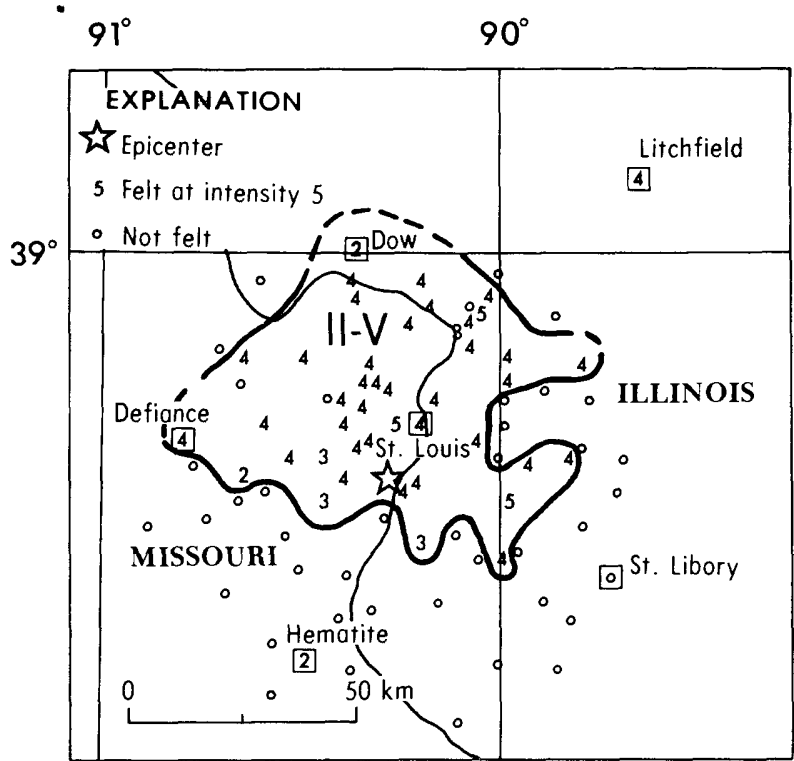

FIGURE 19.--Isoseismal map for the eastern Missouri earthquake of 20 September $1978,12 \quad 24$ 08.8 UTC. Roman numerals represent Modified Mercalli intensities between isoseismals; Arabic numerals are used to represent these intensities at specific sites.

\section{Montana}

14 January (G) Southwestern Montana Origin time: $\quad 165350.3$

Epicenter: $\quad 44.77 \mathrm{~N} ., 111.94 \mathrm{~W}$. Depth :

Magnitude: $\quad 3.6 \mathrm{ML}$

Intensity II: Lake Village (Yellowstone National Park).

23 April (G) Western Montana

Origin time: 232437.0

Epicenter: $\quad 46.97 \mathrm{~N} ., 113.27 \mathrm{~W}$.

Depth:

Magnitude:

$$
5 \mathrm{~km}
$$

$4.5 \mathrm{mb}, 4.3 \mathrm{MS}$,

$$
\text { 4.9 ML }
$$

This earthquake was felt over an area of approximately $94,000 \mathrm{sq}$ $\mathrm{km}$ of eastern Idaho and western Montana ( $f i g .20$ ).

Intensity $\mathrm{V}$ :

Montana-ovando (hanging pictures fell), Ravalli (small objects overturned).

Intensity IV:

Idaho--Calder, North Fork.

Montana--Anaconda, Arlee,

Augusta, Big Arm, Black Eagle, Bonner, Boulder, Butte, Canyon Creek, Carter, Charlo, Choteau, Clancy, Clinton, Conrad, Corvallis, Deer Lodge, Dixon, East

\section{Montana--Continued}

Helena, Elliston, Fairfield, Florence, Frenchtown, Garrison, Great Falls, Greenough, Hall, Hamilton, Helena, Helmville, Hot Springs, Jefferson City, Kalispell, Lincoln, Lolo, Marysville, Milltown, Missoula, Moiese, Monarch, Montana City, oilmont, Pablo, Paradise, Pendroy, Philipsburg, polson, Power, Radersburg, Rollins, Ronan, Saint Ignatius, Saint Reqis, Saltese, Seeley Lake, Silver Star, Simms, Sunburst, Sun River, Superior, Swan Lake, Toston, Townsend, Trout Creek, Troy, Ulm, Valier, Victor, Warmsprings, Whitehall, White Sulphur springs, Winston.

Intensity III:

Idaho--Elk River, Headquarters. Montana--Basin, Brady, Cascade, Darby, Dutton, Essex, Fort Shaw, Jackson, Kevin, Orofino, Raynesford, Stockett, Sula, Vaughn, Willow Creek, Wolf Creek.

Intensity II:

Idaho--Kellogg.

Montaina--Fort Benton, Heart Butte, Polaris, Sand Coulee, Wise River, Wisdom.

23 April (G) Western Montana Origin time: $\quad 233643.7$ Epicenter: $\quad 47.00 \mathrm{~N}, 113.31 \mathrm{~W}$. Depth : Magnitude: $\quad 3.7 \mathrm{MI}$ Intensity II: Dixon, Polson (telephone report).

7 October (G) Western Montana Origin time: $\quad 123555.3$ Fpicenter: $\quad 46.62 \mathrm{~N} ., 112.13 \mathrm{~W}$. Depth: $5 \mathrm{~km}$

Magnitude: $\quad 2.7 \mathrm{ML}(\mathrm{G}), 2.9 \mathrm{ML}(\mathrm{D})$ Intensity IV: East. Helena, Helena.

16 October (G) Western Montana origin time: 011535.3 Epicenter: $\quad 47.09$ N., $113.16 \mathrm{~W}$. Depth : Magnitude: $\quad 2.9 \mathrm{ML}(\mathrm{n}), 3.4 \mathrm{ML}$ Intensity II: Missoula, Potomac (telephone report).

21 october (G) Western Montana

origin time: $\quad 122815.3$

Epicenter: $\quad 46.97 \mathrm{~N},, 113.25 \mathrm{~W}$.

Depth :

Magnitude:

$5 \mathrm{~km}$

29 October (G) Central Idaho

origin time: 134644.5

See Idaho listing. 


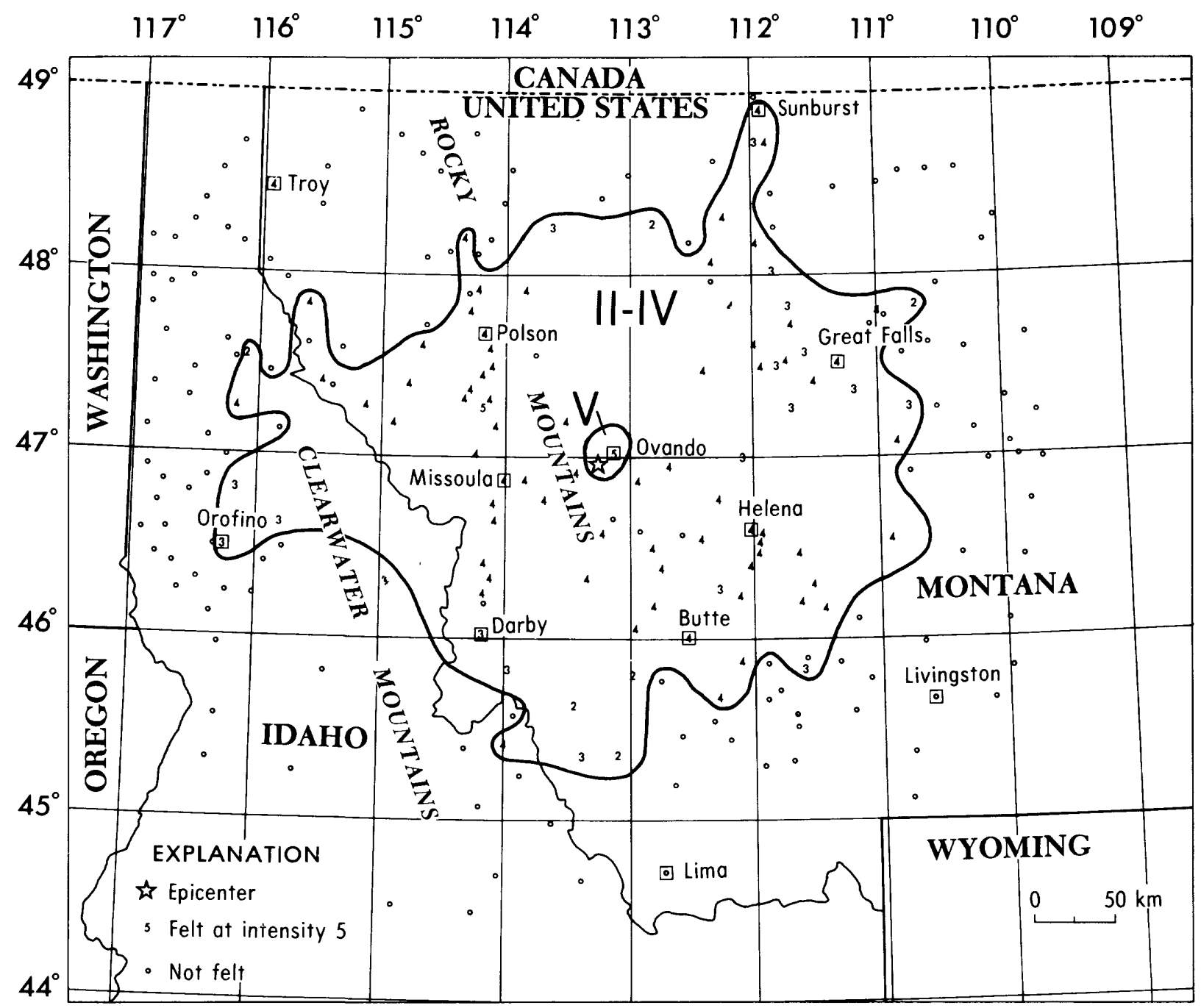

FIGURE 20.--Isoseismal map for the western Montana earthquake of 23 April 1978,2324 37.0 UTC. Roman numerals represent Modified Mercalli intensities between isoseismals; Arabic numerals are used to represent these intensities at specific sites.

Montana--Continued

4 November (G) Hebgen Lake region

origin time: $\quad 154943.8$

Epicenter: $\quad 44.75 \mathrm{~N}, 111.23 \mathrm{~W}$.

Depth: $\quad 5 \mathrm{~km}$

Magnitude: $\quad 3.5 \mathrm{ML}(\mathrm{G})$

Intensity III:

Wyoming--Old Faithful, Yellowstone National Park.

10 November (G) Western Montana

origin time: 095345.6

Epicenter: $\quad 47.01 \mathrm{~N} .113 .33 \mathrm{~W}$.

Depth:

Magnitude:

$5 \mathrm{~km}$

Intensity IV: Seeley Lake

Intensity III : Ovando.

\section{Nebraska}

7 May (G) Central Nebraska

$\begin{array}{ll}\text { Origin time: } & 160619.6 \\ \text { Epicenter: } & 42.30 \mathrm{~N}, 101.93 \mathrm{~W} . \\ \text { Depth: } & 15 \mathrm{~km}\end{array}$

Depth:

Magnitude: $\quad 4.3 \mathrm{mbLg}, 4.0 \mathrm{mbLg}(\mathrm{S})$

Intensity IV: Arthur, Ashby, $27 \mathrm{~km}$ north and $5 \mathrm{~km}$ east of Ashby, $27 \mathrm{~km}$ north of Ashby, $26 \mathrm{~km}$ north of Ashby, $16 \mathrm{~km}$ north of Ashby, $10 \mathrm{~km}$ south of Ashby, $26 \mathrm{~km}$ south of Ashby, $27 \mathrm{~km}$ north of Bingham, 60 $\mathrm{km}$ west of Hyannis, $13 \mathrm{~km}$ southwest of Lewellen, $13 \mathrm{~km}$ north of Mullen, Whitman, $37 \mathrm{~km}$ north of Whitman.

Intensity III: Elsie, $56 \mathrm{~km}$ north of Hyannis, $40 \mathrm{~km}$ north-northeast of Mullen, $5 \mathrm{~km}$ west of Oshkosh. 


\section{Nevada}

13 January (G) Southern Nevada

Origin time: $\quad 033937.0$

Epicenter: $\quad 39.42 \mathrm{~N} ., 117.60 \mathrm{~W}$.

Depth: $5 \mathrm{~km}$

Magnitude: $\quad 4.1 \mathrm{ML}(\mathrm{B})$

Intensity $V$ : Austin.

14 February (G) Southern Nevada

Origin time: 043524.0

Epicenter: $\quad 39.63 \mathrm{~N} ., 117.18 \mathrm{~W}$.

Depth: $\quad 5 \mathrm{~km}$

Magnitude: $\quad 4.4 \mathrm{mb}, 4.8 \mathrm{ML}(\mathrm{B})$

Intensity IV: Austin, Eureka,

Gabbs, Round Mountain, Yerington. Intensity II: Manhattan.

23 February (E) Southern Nevada

origin time: $\quad 170000.164$

Epicenter: $\quad 37.12 \mathrm{~N} ., 116.06 \mathrm{~W}$.

Depth:

Magnitude: $\quad 5.7 \mathrm{mb}(\mathrm{G}), 5.4 \mathrm{ML}(\mathrm{B})$

Nevada Test Site explosion "REBLOCHON" at $37^{\circ} 07^{\prime} 25.24^{\prime \prime} \mathrm{N}$.,

$116^{\circ} 03^{\prime} 49.79^{\prime \prime}$ W. surface elevation $1288 \mathrm{~m}$, depth of burial $658 \mathrm{~m}$.

5 March (G) Northern Nevada

origin time: $\quad 224618.2$

Epicenter: $\quad 38.94$ N., $118.03 \mathrm{~W}$.

Depth:

Magnitude:

$4.0 \mathrm{mb}, 4.6 \mathrm{ML}(\mathrm{B})$

Intensity V: Gabbs (small objects fell).

15 March (G) Western Nevada

Origin time: 030914.9

Epicenter: $\quad 38.35$ N., $118.22 \mathrm{~W}$.

Depth: $\quad 5 \mathrm{~km}$

Magnitude: $\quad 3.2 \mathrm{ML}$

Intensity IV: Gabbs, Luning, Mina.

23 March (E) Southern Nevada

Origin time: $\quad 163000.200$

Epicenter: $\quad 37.10$ N., $116.05 \mathrm{~W}$.

Depth: $0 \mathrm{~km}$

Magnitude: $\quad 5.7 \mathrm{mb}(\mathrm{G}), 5.5 \mathrm{ML}(\mathrm{B})$

Nevada Test Site explosion "ICEBERG" at $37^{\circ} 06^{\prime} 06.39^{\prime \prime}$ W., $116^{\circ} 03^{\prime} 04.13^{\prime \prime}$

W. surface elevation $1266 \mathrm{~m}$, depth of burial $640 \mathrm{~m}$.

11 April (E) Southern Nevada

Origin time: $\quad 153000.161$

Epicenter: $\quad 37.30$ N. $116.33 \mathrm{~W}$.

Depth:

$0 \mathrm{~km}$

Maqnitude :

$5.3 \mathrm{mb}(\mathrm{G}), 5.3 \mathrm{ML}(\mathrm{B})$

Nevada Test Site explosion "FONDOTTA" at $37^{\circ} 17^{\prime} 58.66^{\prime \prime}$ N., 116०19'36.12" W. , surface elevation $2099 \mathrm{~m}$, depth of burial $633 \mathrm{~m}$.

11 April (E) Southern Nevada

Origin time: $\quad 174500.073$

Epicenter: $\quad 37.23 \mathrm{~N} ., 116.37 \mathrm{~W}$.

Depth:

$0 \mathrm{~km}$
Nevada--Continued

Magnitude :

$5.5 \mathrm{mb}(\mathrm{G}), 4.5 \mathrm{MS}(\mathrm{G})$, $5.3 \mathrm{ML}(\mathrm{B})$

Nevada Test Site explosion "BACKBEACH" at $37^{\circ} 14^{\prime} 00.57^{\prime \prime} \mathrm{N}$. $116^{\circ} 22^{\prime} 06.49^{\prime \prime} \mathrm{W}$. , surface elevation $2066 \mathrm{~m}$, depth of burial $611 \mathrm{~m}$.

20 April (G) Southern Nevada

origin time: $\quad 131547.3$

Epicenter: $\quad 35.80 \mathrm{~N} ., 114.90 \mathrm{~W}$.

Depth:

Magnitude:

$10 \mathrm{~km}$

Intensity II: Boulder City.

20 April (G) Southern Nevada

Origin time: 131844.5

Epicenter: $\quad 35.80 \mathrm{~N} ., 114.90 \mathrm{~W}$.

Depth:

Magnitude: $\quad 1.9 \mathrm{ML}$

Intensity II: Boulder City.

23 May (G) Northern Nevada

Origin time: $\quad 054755.4$

Epicenter: $\quad 40.87 \mathrm{~N} .117 .26 \mathrm{~W}$.

Depth :

Magnitude: $\quad 4.1 \mathrm{mb}, 4.6 \mathrm{ML}(\mathrm{B})$

Intensity $V$ : Battle Mountain,

valmy.

Intensity IV: Golconda, Winnemucca.

6 July (G) Northern Nevada

Origin time: 222122.0

Epicenter: $\quad 39.11 \mathrm{~N} ., 116.22 \mathrm{~W}$.

Depth:

Magnitude :

$10 \mathrm{~km}$

. 8 ML

12 July (E) Southern Nevada

origin time: $\quad 170000.075$

Epicenter: $\quad 37.079 \mathrm{~N} ., 116.044 \mathrm{~W}$. Depth: $0 \mathrm{~km}$

Magnitude: $\quad 5.5 \mathrm{mb}(\mathrm{G}), 4.1 \mathrm{MS}(\mathrm{G})$, $5.4 \mathrm{ML}(\mathrm{B})$

Nevada Test Site explosion "LOWBALL" at $37^{\circ} 04 \cdot 43.21^{\prime \prime}$ N., $116^{\circ} 02 \cdot 37.63^{\prime \prime}$

w. , surface elevation $1252 \mathrm{~m}$, depth of burial $564 \mathrm{~m}$.

29 July (G) Central Nevada

Origin time: 223207.1

Epicenter: $\quad 38.40 \mathrm{~N} .115 .24 \mathrm{~W}$.

Depth :

Magnitude: $\quad 3.9 \mathrm{mb}, 4.2 \mathrm{ML}$

Intensity II: Ely (R), Eureka.

31 August (E) Southern Nevada

Origin time: $\quad 140000.164$

Epicenter: $\quad 37.28 \mathrm{~N} .116 .36 \mathrm{~W}$.

Depth:

Magnitude: $\quad 5.6 \mathrm{mb}(\mathrm{G}), 5.5 \mathrm{ML}(\mathrm{B})$

Nevada Test Site explosion "PANIR" at $37^{\circ} 16^{\prime} 33.34^{\prime \prime}$ N., $116^{\circ} 21^{\prime} 26.43^{\prime \prime} \mathrm{W}$. , surface elevation $2040 \mathrm{~m}$, depth of burial $681 \mathrm{~m}$. 
Nev ada--Continued

4 September (B) Lake Tahoe region origin time: 045231.6

See California listing.

4 September (B) Lake Tahoe region origin time: $\quad 215452.5$

See California listing.

4 October (P) Owens Valley area origin time: $\quad 164248.6$

See California listing.

13 September (E) Southern Nevada Origin time: $\quad 151500.161$ Epicenter: $\quad 37.21 \mathrm{~N}, 116.21 \mathrm{~W}$. Depth: Magnitude: $\quad 4.6 \mathrm{mb}(\mathrm{G}), 4.6 \mathrm{ML}(\mathrm{B})$

Nevada Test Site explosion "DIABLO HAWK" at $37^{\circ} 12^{\prime} 31.68^{\prime \prime} \mathrm{N}$. $116^{\circ} 12^{\prime} 38.78^{\prime \prime} \mathrm{W} .$, surface elevation $2239 \mathrm{~m}$, depth of burial $388 \mathrm{~m}$.,

27 September (E) Southern Nevada

$\begin{array}{ll}\text { Origin time: } & 170000.071 \\ \text { Epicenter: } & 37.07 \mathrm{~N} ., 116.02 \mathrm{~W} \\ \text { Depth: } & 0 \mathrm{~km}\end{array}$

Magnitude: $\quad 5.0 \mathrm{mb}(\mathrm{G}), \quad 5.0 \mathrm{ML}(\mathrm{B})$

Nevada Test Site explosion "DRAUGHTS" at $37^{\circ} 04^{\prime} 25.92^{\prime \prime}$ N., 116 $01^{\circ} 11.40^{\prime \prime}$ W. surface elevation $1253 \mathrm{~m}$, depth of burial $442 \mathrm{~m}$.

27 September (E) Southern Nevada

origin time: $\quad 172000.076$

Epicenter: $\quad 37.08 \mathrm{~N} .116 .05 \mathrm{~W}$.

Depth :

$0 \mathrm{~km}$

Magnitude:

$5.7 \mathrm{mb}(\mathrm{G}), 4.1 \mathrm{MS}(\mathrm{G})$, $5.5 \mathrm{ML}(\mathrm{B})$

Nevada Test Site explosion "RUMMY" at $37^{\circ} 04^{\prime} 47.29^{\prime \prime}$ N., 116 $16^{\circ} 03^{\prime} 04.76^{\prime \prime} \mathrm{W}$. , surface elevation $1262 \mathrm{~m}$, depth of burial $640 \mathrm{~m}$.

2 November (E) Southern Nevada

origin time: $\quad 152500.169$

Epicenter: $\quad 37.29 \mathrm{~N} ., 116.30 \mathrm{~W}$.

Depth: $\quad 0 \mathrm{~km}$

Magnitude: $\quad 4.2 \mathrm{mb}(\mathrm{G}), 4.3 \mathrm{ML}(\mathrm{B})$

Nevada Test Site explosion "FMMENTHAL" at $37^{\circ} 17^{\prime} 16.60 " \mathrm{~N}$. $116^{\circ} 17^{\prime} 50.98^{\prime \prime} \mathrm{W}$., surface elevation $2131 \mathrm{~m}$, depth of burial $576 \mathrm{~m}$.

18 November (E) Southern Nevada

origin time: $\quad 190000.166$

Epicenter: $\quad 37.13 \mathrm{~N} ., 116.08 \mathrm{~W}$.

Depth:

$0 \mathrm{~km}$

Maqnitude: $\quad 5.1 \mathrm{mb}(\mathrm{G}), 5.2 \mathrm{ML}(\mathrm{B})$

Nevada Test Site explosion "OUARGEL" at $37^{\circ} 07^{\prime} 36.73^{\prime \prime}$ N. $116^{\circ} 05^{\prime} 01.94^{\prime \prime}$
Nevada--Continued

W. , surface elevation $1302 \mathrm{~m}$, depth of burial $542 \mathrm{~m}$.

16 December (E) Southern Nevada

Oriqin time: $\quad 153000.158$

Epicenter: $\quad 37.27 \mathrm{~N} ., 116.41 \mathrm{~W}$.

Depth: $0 \mathrm{~km}$

Magnitude: $\quad 5.5 \mathrm{mb}(\mathrm{B}), 5.5 \mathrm{ML}(\mathrm{B})$

Nevada Test Site explosion "FARM" at $37^{\circ} 16^{\prime} 24.22^{\prime \prime}$ N., $116^{\circ} 24^{\prime} 36.99^{\prime \prime}$ W. surface elevation $2006 \mathrm{~m}$, depth of burial $689 \mathrm{~m}$.

New Hampshire

25 August (J) Southeastern New Hampshire origin time: $\quad 200130.5$

Epicenter: $\quad 42.87 \mathrm{~N} ., 70.83 \mathrm{~W}$.

Depth:

Magnitude: $\quad 2.3 \mathrm{mbLg}$

Intensity III: Seabrook (J).

\section{New Jersey}

30 June (L) Northern New Jersey

origin time: 201343.6

Epicenter: $\quad 41.07 \mathrm{~N} ., 74.20 \mathrm{~W}$.

Depth:

Magnitude:

$5 \mathrm{~km}$

Intensity IV:

New Jersey--Mahwah (press report), Oakland (press report).

New York--Hillburn (press report), Suffern (press report).

Intensity III:

New York--Pine Grove (press report), Sloatsburg (press report), Tuxedo Park (press report).

30 June (L) Northern New Jersey

Origin time: 223949.7

Epicenter: $\quad 41.08 \mathrm{~N} .74 .20 \mathrm{~W}$.

Depth: $\quad 6 \mathrm{~km}$

Magnitude: $\quad 2.2 \mathrm{ML}$

Intensity III: Oakland, New JerseySuffern, New York area (press report).

New Mexico

16 June (G) West Texas

origin time: $\quad 114654.2$

See Texas listing.

New York

18 February (O) Southern Quebec origin time: $\quad 144825.0$ 
New York--Cont inued

Epicenter:

Depth:

Magnitude:

Intensity $\mathrm{V}$ :

Quebec--Saint Donat de Montcalm region.

Intensity IV:

New York--Rouses Point.

5 March (L) Southeastern New York

origin time: $\quad 075325.6$

Epicenter :

Depth:

Magnitude:

$41.35 \mathrm{~N} ., 74.15 \mathrm{~W}$.

$5 \mathrm{~km}$

Intensity III: Highland Mills (J).

30 June (L) Northern New Jersey

origin time: $\quad 2013 \quad 43.6$

See New Jersey 1 isting.

30 June (L) Northern New Jersey

origin time: $\quad 223949.7$

See New Jersey 1 isting.

North Carolina

25 February (V) Northern North Carolina

origin time: 035327.7 .

Epicenter: $\quad 36.19 \mathrm{~N} ., 79.30 \mathrm{~W}$.

Depth:

Magnitude:

$8 \mathrm{~km}$

Intensity IV: Burlington.

22 March (V) Northern North Carolina

Origin time: 155226.7

Epicenter: $\quad 36.20 \mathrm{~N} ., 81.73 \mathrm{~W}$.

Depth:

$1 \mathrm{~km}$

Magnitude:

$2.9 \mathrm{mbLg}$

Possible explosion (V).

24 March (G) Southwest of Bermuda Islands origin time: $\quad 004238.2$

See Massachusetts 1 isting.

oklahoma

9 March (T) Southern Oklahoma

origin time: 063051.2

Epicenter: $\quad 34.07 \mathrm{~N} .97 .40 \mathrm{~W}$.

Depth:

Magnitude:

Intensity II: Simon area (T).

17 May ( $T$ ) Central Oklahoma

origin time: 231115.7

Epicenter: $\quad 35.53 \mathrm{~N} .97 .91 \mathrm{~W}$

Depth:

Magnitude:

$5 \mathrm{~km}$

Intensity $\mathrm{I}:$

I: $\quad$ El Reno (T).
oklahoma--Continued

18 May (T) Central Oklahoma

origin time: 001922.4

Epicenter: $\quad 35.50 \mathrm{~N} ., 97.50 \mathrm{~W}$.

Depth: $\quad 5 \mathrm{~km}$

Magnitude: $\quad 2.7 \mathrm{mbLg}$

Intensity III: $\mathrm{El}$ Reno $(\mathrm{T})$.

18 May (T) Central Oklahoma

Origin time: $\quad 003217.6$

Epicenter: $\quad 35.60 \mathrm{~N} ., 97.83 \mathrm{~W}$.

Depth:

$5 \mathrm{~km}$

Magnitude: $\quad 2.1 \mathrm{mbLg}$

Intensity II: $\quad \mathrm{El}$ Reno (T).

Pennsylvania

16 July (G) Southeastern Pennsylvania

origin time: $\quad 063937.8$

Epicenter: $\quad 39.92 \mathrm{~N} ., 76.26 \mathrm{~W}$.

Depth:

Magnitude :

$5 \mathrm{~km}$

$2.9 \mathrm{mbLg}(\mathrm{V})$, $3.1 \mathrm{mbLg}(\mathrm{J})$

This earthquake was felt over an area of approximately $1,600 \mathrm{sq} \mathrm{km}$ of Pennsylvania, Delaware, and Maryland (fig. 21).

Intensity $\mathrm{V}$ :

Pennsylvania--Lancaster (few plaster cracks), New providence (few plaster cracks).

Intensity IV:

Delaware--Wilmington.

Maryland--Cardiff, Pylesville, Whiteford.

Pennsylvania--Airville, Bart, Bausman, Brogue, Buck, Columbia, Conestoga, Craley, East Prospect, Fawn Grove, Gordonville, Holtwood, Kirkwood, Millersville, Mount Nebo, Mountville, Paradise, Peach Bottom, Penryn, Pequea, Rawlinsville, Red Lion, Refton, Smoketown, Strasburg, Washington Boro, Willow street, Windsor, Wrightsville (11 km south).

Intensity III:

Maryland--Darlington.

Pennsylvania--Manhe im.

6 October (G) Southeastern Pennsylvania origin time: $\quad 192541.6$ Epicenter: $\quad 39.97 \mathrm{~N}, 76.51 \mathrm{~W}$. Depth: $\quad 5 \mathrm{~km}$

Magnitude: $\quad 2.8 \mathrm{mbLg}, 3.0 \mathrm{mbLg}(\mathrm{L})$

According to the press, this earthquake shook buildings throughout Lancaster and its suburbs. Buildings trembled briefly, a loud noise (described variously as a boom, roar, or vehicle collision) could be heard, and some startled people ran outside. 


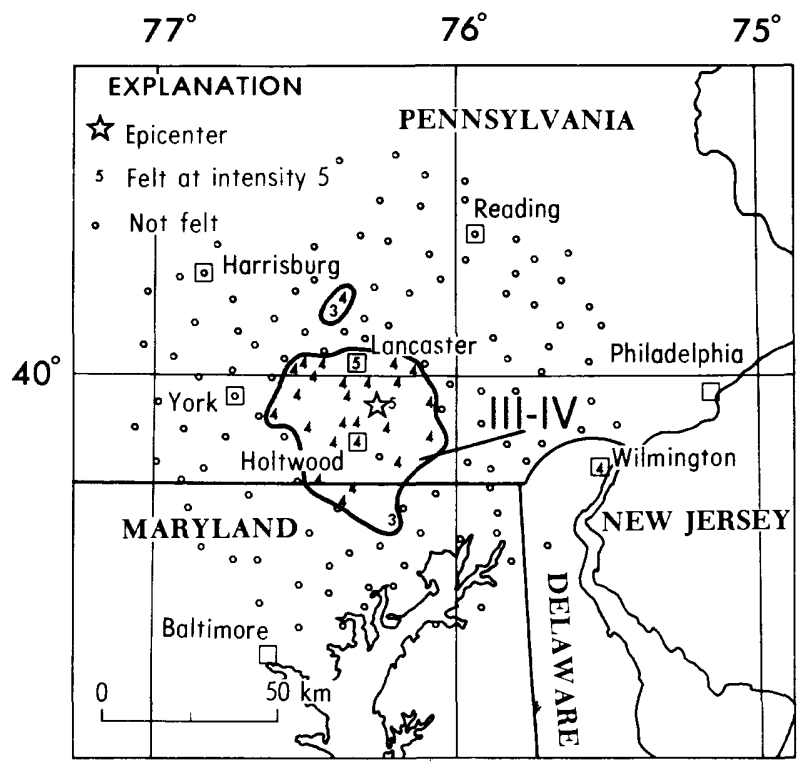

FIGURE 21.--Isoseismal map for the southeastern Pennsylvania earthquake of 16 July 1978 , $\begin{array}{llll}06 & 39 & 37.8 & \text { UTC. Roman }\end{array}$ numerals represent Modified Mercalli intensities between isoseismals; Arabic numerals are used to represent these intensities at specific sites.

Pennsylvania--Conti nued

Intensity $V$ : Brunnerville (few cracked windows), Mount Joy (unconfirmed report of cracked plaster).

Intensity IV: Akron, Bausman, Bird in Hand, Brogue, Brownstown, Conestoga, Delta, East Petersburg, Lancaster, Landisville, Leola, Mountville, New Holland, Peach Bottom, Penryn, Quarryville, Refton, Ronks, Smoketown, Talmage, Washington Boro, Willow Street, Wrightsville.

Intensity III: Intercourse, Millersville, Pequea, strasburg.

Intensity II: Ephrata (press report), Manheim, Marticville (press report), Millersville (press report).

\section{Puerto Rico}

15 May (G) Mono Passage

Origin time:

Epicenter :

Depth :

Magnitude:

$\begin{array}{lll}19 & 25 & 14.1\end{array}$

$18.73 \mathrm{~N} .67 .43 \mathrm{~W}$

Intensity II
South Carolina

7 September (G) Eastern South Carolina origin time: 225322.3

Epicenter: $\quad 33.07 \mathrm{~N},{ }^{2} 80.22 \mathrm{~W}$.

Depth:

Magnitude: $11 \mathrm{~km}$

Intensity IV: Summerville--Corey Woods $(4.2 \mathrm{~km}$ southwest on Highway 17A--windows, doors, and dishes rattled).

Intensity III: Jedburg, Summerville--city area, Summerville--Briarwood $(3.2 \mathrm{~km}$ south on State Highway 199), College Park $(7.8 \mathrm{~km}$ east off Interstate 26), Flowertown Village (5.1 $\mathrm{km}$ southwest on Highway $17 \mathrm{~A}$ ), King's Grant ( $8 \mathrm{~km}$ south on Highway $642)$, Knightsville $(5.8 \mathrm{~km}$ west on Highway 13), Oakdale Subdivision ( 4 $\mathrm{km}$ southwest on Highway 17A), old Orangeburg Road $(5.3 \mathrm{~km}$ west on Highway 22), Owen's Circle $(5.9 \mathrm{~km}$ south on Highway 165), Pine Forest Estates ( $4 \mathrm{~km}$ southwest on Highway 17A), Quail Arbor II (3.8 km south on Highway 165), Quail Arbor V (4.6 $\mathrm{km}$ south on Highway 199), Shepard Park (2.2 km west on Highway 17A), Twin Oaks $(1.8 \mathrm{~km}$ south off Highway 165), Waring Hall $(3.4 \mathrm{~km}$ west on Highway 13), Warrington Subdivision (1.3 km west on Highway 13 ).

Intensity II: Summerville--old Fort Estates $(7.2 \mathrm{~km}$ south on Highway 642 ).

Tennessee

18 January (S) Western Tennessee

Origin time: 234626.1

Epicenter: $\quad 36.25 \mathrm{~N} ., 89.42 \mathrm{~W}$.

Depth :

Magnitude: $\quad 2.6 \mathrm{mbLg}$

Intensity III: Ridgely (Tennessee Earthquake Information Center).

31 August (S) New Madrid, Missouri region Origin time: 003100.3

Epicenter: $\quad 36.09 \mathrm{~N} ., 89.42 \mathrm{~W}$.

Depth:

Magnitude:

$4 \mathrm{~km}$

$3.5 \mathrm{mbLg}$

Intensity V:

Tennessee--Dyersburg (S).

Intensity IV:

Arkansas--Leachville.

Missouri--Bragaadocio, Hayti, Pascola.

Tennessee--Bogota, Findley, Lenox, Troy.

Intensity III:

Arkansas--Etowah.

Missouri--Caruthersville, Portageville.

Tennessee--Memphis, Samburg, Yorkville.

Intensity II: 
Arkansas--Keiser.

Tennessee--Kenton.

\section{Texas}

2 March (G) Southwestern Texas

Origin time: $\quad 100452.7$

Epicenter: $\quad 31.56$ N., $102.51 \mathrm{~W}$.

Depth :

Magnitude:

$11 \mathrm{~km}$

$3.5 \mathrm{ML}$

Intensity III: Kermit (telephone report).

16 June (G) West Texas

$\begin{array}{ll}\text { Origin time: } & 114654.2 \\ \text { Epicenter: } & 33.03 \mathrm{~N}, 100.77 \mathrm{~W} . \\ \text { Depth: } & 10 \mathrm{~km} \\ \text { Magnitude: } & 4.4 \mathrm{mb}, 5.3 \mathrm{ML}, \\ & 4.6 \mathrm{mbLg}(\mathrm{S})\end{array}$

This earthquake was felt over an area of approximately $52,000 \mathrm{sq} \mathrm{km}$ of west Texas (fig. 22).

Intensity V:

Texas-Carlsbad (hanging pictures fell, small objects broke), Fluvanna (one broken window, hanging pictures fell, light furniture shifted), Justiceburg (unconfirmed report of cracked plaster), Peacock (few cracked windows), Rotan (light furniture shiftéd), Snyder (cracked window, mirror fell and broke).

Intensity IV:

New Mexico--Hobbs.

Texas--Abilene, Ackerly, Albany (press report), Anson, Aspermont, Ballinger, Big Lake, Big Spring, Blackwell, Breckenridge, Bronte, Coahoma, Coleman, Clyde, Colorado City, Dermott, Dunn, Eola, Gail, Girard, Goldsboro, Goodfel low AFB, Guthrie, Hamlin, Hermleigh, Ira, Jayton, Knott, Lamesa, Lorenzo, Lubbock, Lueders, Maryneal, McCaulley, Mereta, Merkel, Mertzon, Miles, Moran, New Home, Nolan, Old Glory, Ovalo, Roby, Roscoe, Rule, San Angelo, San Angelo--Angelo State University, Spur, Stamford, Stanton, Sylvester, Sweetwater (press report), Tahoka, Tuscola (press report), Valdera, Veribest, Water Valley, Westbrook, windgate, winters, Woodson.

Intensity III:

Texas--Gouldbusk, Loraine, Talpa. Intensity II:

Texas--Glen Cove, Post, Sagerton.
28 February (U) Northern Utah

Origin time: $\quad 002006.5$

Epicenter: $\quad 40.76 \mathrm{~N} ., 112.20 \mathrm{~W}$.

Depth:

Magnitude :

$14 \mathrm{~km}$

Intensity IV: Magna, Salt Lake City.

Intensity III: Tooele.

Intensity II: Farmington.

9 March (U) Northern Utah

Origin time: 063051.8

Epicenter: $\quad 40.76 \mathrm{~N} ., 112.08 \mathrm{~W}$.

Depth :

Magnitude: $\quad 3.3 \mathrm{ML}$

Intensity VI: Magna (cracks in the exterior wall of fire station no. 2 ; windows broken, plaster and dry wall cracked).

Intensity V: Salt Lake City.

Intensity IV: Hooper, Murray.

Intensity III: Centerville (press report), Lincoln, Midvale.

9 March (U) Northern Utah

Origin time: 064620.0

Epicenter: $\quad 40.77 \mathrm{~N} ., 112.08 \mathrm{~W}$.

Depth:

Magnitude:

$7 \mathrm{~km}$

Intensity IV: Magna (press report).

13 March (U) Northern Utah

Origin time: $\quad 133543.4$

Epicenter: $\quad 40.76 \mathrm{~N}, 112.08 \mathrm{~W}$.

Depth :

Magnitude:

$13 \mathrm{~km}$

Intensity III: Bountiful (press report), Granger (press report), Salt Lake City (Glendale area and University of Utah Hospital--press report).

Intensity II: Midvale.

29 July (U) Northern Utah

Origin time: $\quad 140403.2$

Epicenter: $\quad 41.85 \mathrm{~N}, 112.13 \mathrm{~W}$.

Depth:

Magnitude: $\quad 3.1 \mathrm{ML}$

Intensity IV:

Idaho--Weston.

Utah--Cornish, Fielding, Howell, Logan, Newton, Plymouth, Portage, Preston, Richmond, Riverside, Smithfield, Trenton.

Intensity III:

Idaho--Frankl in.

Utah--Cherry Creek, Collinston, Garland.

Intensity II:

Idaho-Dayton.

Utah--Clarkston.

24 october (U) Southeastern Idaho origin time: $\quad 203059.3$

see Idaho listing. 


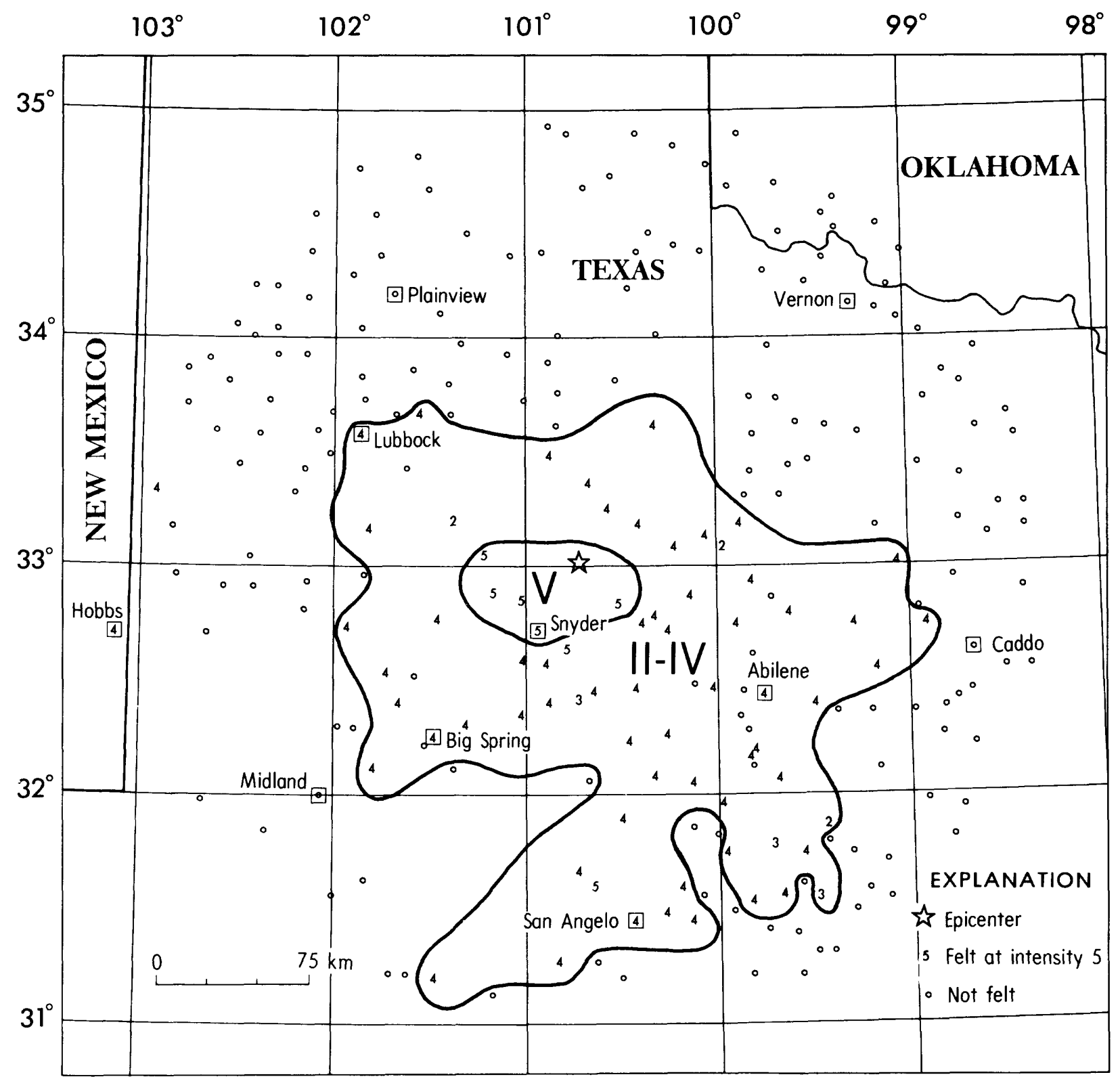

FIGURE 22.--Isoseismal map for the west Texas earthquake of 16 June $1978,114654.2$ UTC. Roman numerals represent Modified Mercalli intensities between isoseismals; Arabic numerals are used to represent these intensities at specific sites.

Utah--Cont inued

30 November (U) Southeastern Idaho origin time: 065340.1

See Idaho listing.

20 December (U) Southeastern Idaho origin time: $\quad 134622.6$

see Idaho listing.

\section{Virginia}

17 March (V) Southwestern Virginia Origin time: $\quad \begin{array}{lll}18 & 26 & 34.5\end{array}$ Fpicenter: $\quad 36.75$ N. , $80.74 \mathrm{~W}$. Depth:

Magnitude: $7 \mathrm{~km}$

Intensity IV: Galax, Hillsville, Lambsburg, Laurel Fork, Woodlawn. Intensity III: Dugspur.

Intensity II: Austinville, Indepen- 
5 March (W) Puget Sound area

origin time: 181335.9

Epicenter: $\quad 48.06 \mathrm{~N} ., 123.00 \mathrm{~W}$.

Depth :

Magnitude:

Intensity IV:

$57 \mathrm{~km}$

$4.0 \mathrm{mb}(\mathrm{G}), 3.3 \mathrm{ML}(\mathrm{G})$

Intensity III:

British Columbia--Victoria (telephone report).

washington--North Seattle (telephone report), Oak Harbor (telephone report).

Intensity II: Hansville.

11 March (W) Puget Sound area

origin time: $\quad 155211.2$

Epicenter: $\quad 47.42$ N., $122.71 \mathrm{~W}$.

Depth :

Magnitude:

$25 \mathrm{~km}$

$4.3 \mathrm{mb}(\mathrm{G}), 4.8 \mathrm{ML}$, $3.2 \mathrm{MS}(\mathrm{G})$

This earthquake was felt over an area of $20,000 \mathrm{sq} \mathrm{km}$ of northwest Washington ( $f i g .23$ ).

Intensity VI: Crystal Mountain Ski Resort (crack in rock and mortar wall at the Summit House; open beams supporting the roof moved 1-2 cm), Freeland (cracked plaster), Grotto (cracked plaster), Longbranch (cracked plaster and windows ).

Intensity V: Ashford, Bremerton, Centralia, Dockton, Enumclaw, Everett, Federal Way, Hobart, Issaquah, La Grande, Leavenworth, Olympia, Port Orchard, Poulsbo, Preston, Redondo, Ronald, Seabeck, seattle, snoqualmie Pass, South Colby, Tacoma.

Intensity IV: Aberdeen, Allyn, Anderson Island, Baring, Belfair, Blakely Island, Brinnon, Burley, Cinebar, Clearlake, Des Moines, East olympia, Eatonville, Fort Lewis (Gray Army Airfield), Fox Island, Gig Harbor, Glenoma, Hadlock, Hoodsport, Indianola, Kapowsin, Kent, La Conner, Lakebay, Little Rock, Lyman, Lynnwood, McKenna, Medina, Milton, Mukilteo, olalla, olga, Orting, Pacific, Parkland, Port Angeles, Proctor, Puyallup, Ranier, Renton, Roy, Skykomish, Spanaway, Tillicum, Tracyton, Union, Vashon, Vaughn, wilkeson, Winslow, Yelon.

Intensity III: Buckley, Carbonado, Carnation, Fircrest, Fort Lewis, Gold Bar, Lake Stevens, Lakewood Center, McMillin, Napavine, Oakville, Randle, Redmond, Wallingford, Wenatchee (press report).

Intensity II: Bellevue, Edmonds, Fall City, Forest Park, Index, Lester, Lilliwaup, Mineral.
29 March (W) Northwest Washington

Origin time: $\quad 121638.4$

Epicenter: $\quad 48.20 \mathrm{~N},, 122.76 \mathrm{~W}$.

Depth:

Magnitude:

$24 \mathrm{~km}$

Intensity IV: Oak Harbor.

31 March (W) Puget Sound area

origin time: 080300.2

Epicenter: $\quad 47.42 \mathrm{~N} ., 122.72 \mathrm{~W}$.

Depth:

Magnitude: $\quad 4.2 \mathrm{ML}(\mathrm{G})$

This earthquake was felt over an area of $7,000 \mathrm{sq} \mathrm{km}$ of the Puget Sound area (fig. 24).

Intensity $\mathrm{V}$ : Bremerton (hairline cracks in exterior walls), Brinnon, Chimacum, Hadlock, Manchester, Olalla, Port Orchard, Port Ludlow, Poulsbo, Quilcene, Rollingbay, Seabeck, Seattle, Tacoma, Tracyton, Union.

Intensity IV: Auburn, Baring, Belfair, Bellevue, Burley, Crystal Mountain, Dockton, Fircrest, Fox Island, Hansville, Index, Indianola, Kapowsin, Kingston, Lilliwaup, Matlock, Medina, Olympia, Puyallup, Ravensdale, Tahuya, Vaughn.

Intensity III: La Grande. Intensity II: Carnation, Nordland.

27 June (W) Washington

Origin time: 021858.8

Epicenter: $\quad 46.86 \mathrm{~N} ., 120.96 \mathrm{~W}$.

Depth: $1 \mathrm{~km}$

Magnitude: $\quad 3.7 \mathrm{ML}(\mathrm{G})$

Intensity II: Naches Ranger Station (W), Snoqualmie.

23 August (Q) Vancouver Island, British Columbia

Origin time: $\quad 103718.0$

Epicenter: $\quad 48.38 \mathrm{~N} ., 123.20 \mathrm{~W}$.

Depth:

$17 \mathrm{~km}$

Magnitude: $\quad 4.4 \mathrm{mb}(\mathrm{G}), 3.5 \mathrm{ML}$

Felt at intensity $V$ on Vancouver Island.

Intensity IV: Eastsound, Friday Harbor.

Intensity II: Port Angeles (W).

31 December (W) Seattle area

origin time: $\quad 032346.7$

Epicenter: $\quad 47.58 \mathrm{~N}, 121.85 \mathrm{~W}$.

Depth :

$20 \mathrm{~km}$

Magnitude: $\quad 4.0 \mathrm{ML}(\mathrm{G})$

This earthquake was felt over an area of approximately $8,500 \mathrm{sq} \mathrm{km}$ of Washington and British Columbia (fig. 25). 


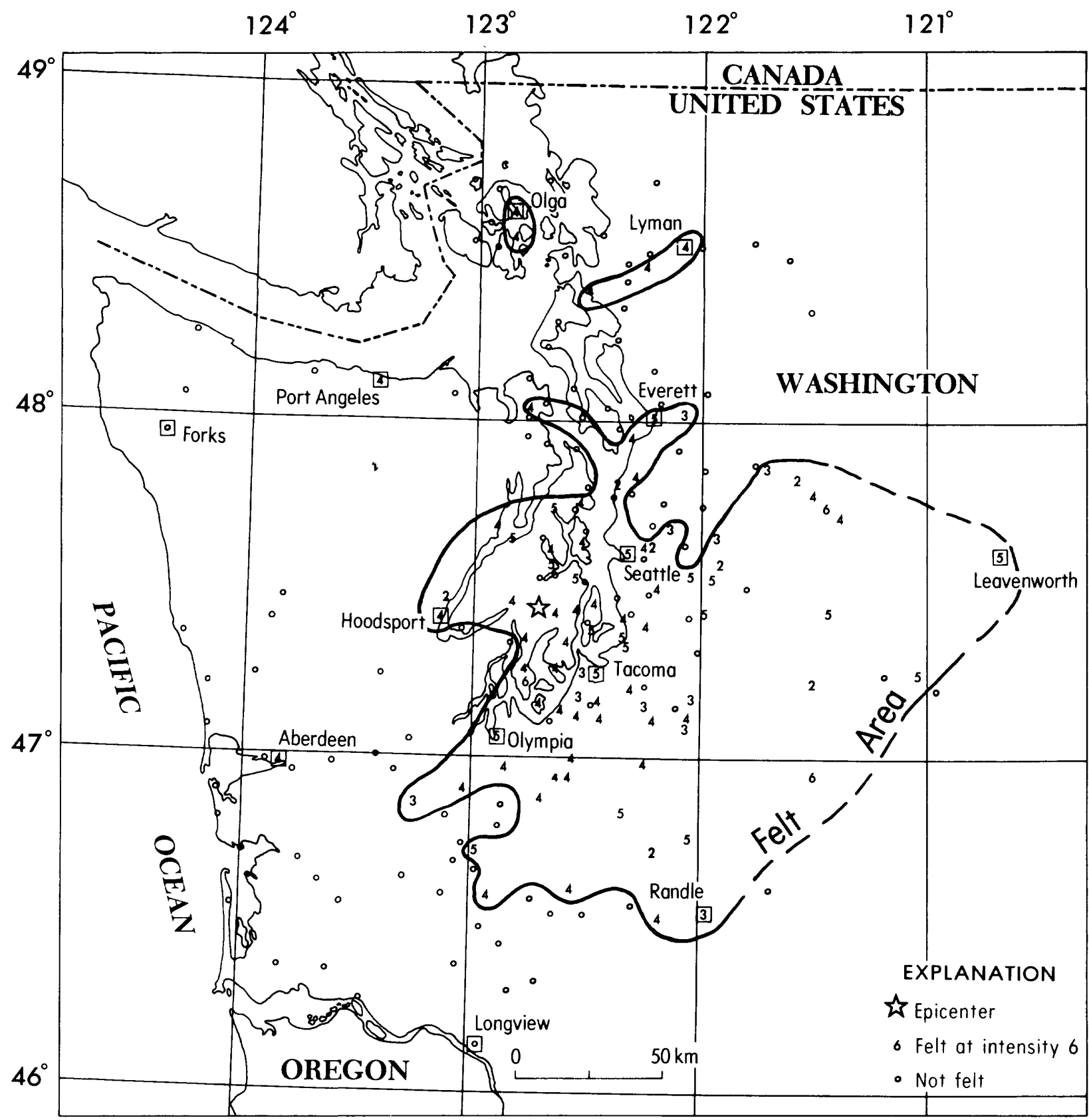

FIGURE 23.--Isoseismal map for the Puget Sound, Washington, earthquake of 11 March 1978, 155211.2 UTC. Roman numerals represent Modified Mercalli intensities between isoseismals; Arabic numerals are used to represent these intensities at specific sites. Dashed line indicates the isoseismal is not well defined by the data.

Intensity $\mathrm{V}$ :

Washington--

Granite Falls (light furniture and small objects shifted, felt by many),

North Seattle (press reported small cracks in walls and ceil- ing of one home).

Intensity IV:

Washington--Bothell, Clinton, Fall City, Freeland, Gold Bar, Grotto, Hobart, Index, Kenmore (press report), Kingston, Lake City (press report), Lake Forest Park 


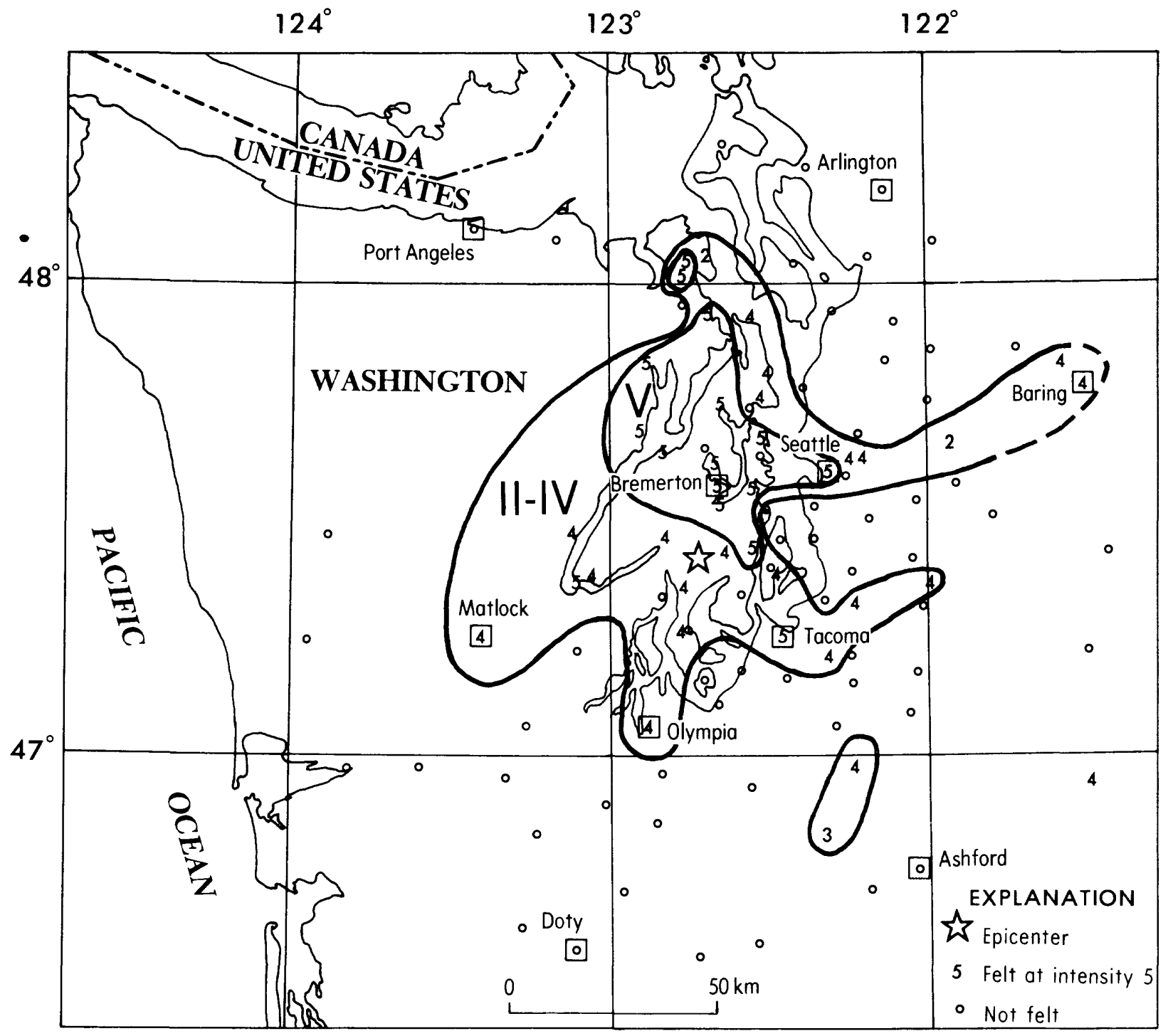

FIGURE 24.--Isoseismal map for the Puget Sound, Washington, earthquake of 31 March 1978, 080300.2 UTC. Roman numerals represent Modified Mercalli intensities between isoseismals; Arabic numerals are used to represent these intensities at specific sites. Dashed line indicates the isoseismal is not well defined by the data.

Washington--Continued

(press report), Lake Stevens, Magnolia (press report), Monroe, Mukilteo, Northbend, Port Orchard, Preston, Ravensdale, Renton, seattle, Seattle-Tacoma Airport (furniture shifted on the fifth floor), Snoqualmie, Sultan, Woodinville.

Intensity III:

Washington--Belfair, Conway, Darrington, Everett, Kirkland, Medina, Mountlake Terrace, Port Townsend, Shaw Island, Startup.
Washington--Continued

British Columbia--Victoria (press report).

Washington--Black Diamond, Coupeville, Edmonds, North Bend, Retsil, Skykomish, Tacoma.

\section{Wyom ing}

13 January

Yellowstone National Park

Seven earthquakes were felt in the eastern part of the park from Janu- 


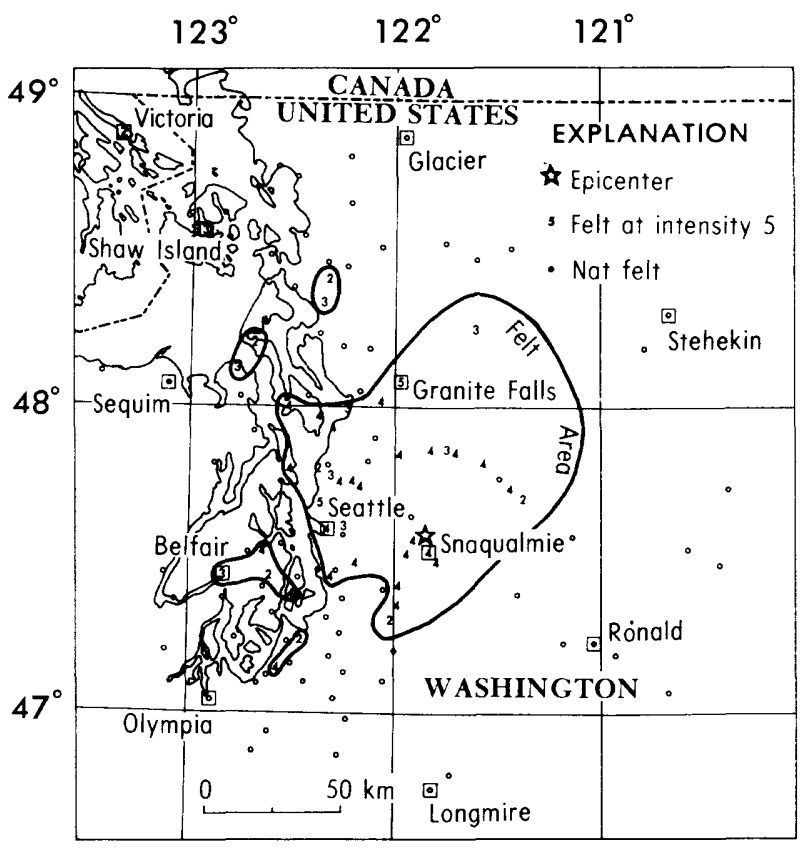

FIGURE 25.--Isoseismal map for the Seattle earthquake of 31 December $1978,032346.7 \mathrm{UTC}$. Roman numerals represent Modified Mercalli intensities between isoseismals; Arabic numerals are used to represent these intensities at specific sites.

Wyoming--Continued

ary 13 to 21 . The largest magnitude of these quakes was about 1.8 ML. They were felt at Canyon Village and Lake, with the smaller magnitude events only being felt at Lake. Maximum intensity was IV.

16 January (G) Southeast Wyoming

Origin time: 035003.1

Epicenter: $\quad 42.43 \mathrm{~N} ., 105.31 \mathrm{~W}$.

Depth:

Magnitude:

$5 \mathrm{~km}$

3. $0 \mathrm{ML}$

Intensity III: $\quad 14.5 \mathrm{~km}$ north of Laramie, Wheatland (telephone report).

2 February (G) Yellowstone National Park origin time: $\quad 003625.6$

Epicenter: $\quad 44.39 \mathrm{~N} ., 110.81 \mathrm{~W}$.

Depth:

$5 \mathrm{~km}$

Maqnitude: $\quad 3.6 \mathrm{mb}, 3.4 \mathrm{ML}$, 3. $6 \mathrm{ML}(\mathrm{D})$

Intensity III: Old Faithful.

(Shocks at on 4901.4 and 0430

25.9 with magnitudes of $2.8 \mathrm{ML}$ and

2.6 ML were also felt.)

2 February (G) Yellowstone National Park origin time: $\quad 123556.2$

Epicenter: $\quad 44.38 \mathrm{~N} ., 110.83 \mathrm{~W}$.
Wyoming--Continued

Depth:

Magnitude:

$4 \mathrm{~km}$

Intensity III: old Faithful.

7 March (G) Yellowstone National Park origin time: $\quad 011047.6$

Epicenter: $\quad 44.43 \mathrm{~N} ., 110.84 \mathrm{~W}$.

Depth:

$5 \mathrm{~km}$

Magnitude:

$3.7 \mathrm{mb}, 3.5 \mathrm{ML}$, $3.8 \mathrm{ML}(\mathrm{D})$

From March 7 at 0109 UTC, to 1830 UTC, a swarm of 129 tremors was felt at old Faithful. This series was known to have immediately induced minor changes in the thermal activity in the Upper Geyser Basin. These included increased turbidity in Heart Spring and Calida, and more vigorous splashing in Tea Kettle, with a rise in water level.

No changes in thermal activity occurred at the Norris Geyser Basin for 2.5 days after the swarm. But by March 23, Palpitar Spring had drained and the algae had died. At Steamboat Geyser minor eruptions appeared to have greater volume, and on March 28 the geyser had its first major eruption in 9 years. Runoff and sinter deposition at Cistun Springs had shifted from the west side of the pool to the southeast side, where two small trees were dying from the hot water (R. A. Hutchinson, National Park Service, written commun., 1978).

Intensity V: old Faithful.

7 March (G) Yellowstone National Park Origin time: 073933.1 Epicenter: $\quad 44.30 \mathrm{~N} ., 110.92 \mathrm{~W}$. Depth: $\quad 5 \mathrm{~km}$ Magnitude: $\quad 3.8 \mathrm{mb}, 3.1 \mathrm{ML}$ Intensity IV: Old Faithful.

15 April (G) Western Wyoming origin time: 052350.8 Fpicenter: $\quad 42.72 \mathrm{~N}, 110.88 \mathrm{~W}$. Depth: Magnitude: $5 \mathrm{~km}$ Intensity II: Afton.

7 June Yellowstone National Park origin time: 1228 Fpicenter: Not located. Depth: None computed. Magnitude: None computed. Intensity IV: Canyon Village. Intensity III : Lake.

8 June Yellowstone National Park origin time: $\quad 150154$ Epicenter: Not located. Depth: None computed. 
Magnitude: None computed.

Intensity III: Old Faithful.

18 June Yellowstone National Park

origin time: 2240

Epicenter: Not located.

Depth: None computed.

Magnitude: None computed.

Intensity III: old Faithful.

19 June Yellowstone National Park

origin time: 0543

Epicenter: Not located.

Depth: None computed.

Magnitude: None computed.

Intensity IV: Lake-Fishing Bridge area.

15 July Yellowstone National Park

Origin time: 0826

Epicenter: Not located.

Depth: None computed.

Magnitude: None computed.

Intensity IV: Norris.

Intensity III: Canyon Village.

21 July Yellowstone National Park Origin time: $\quad 1001$

Epicenter: Not located.

Depth: None compured.

Magnitude: None computed.

Intensity IV: Grant Village.

21 August Yellowstone National Park

origin time: 1214

Epicenter: Not located.

Depth: None computed.

Magnitude: None computed.

Intensity III: Old Faithful (earthquakes at $12: 16,12: 27$, and $12: 32$ were also felt at intensity III).

21 August Yellowstone National Park

origin time: 1314

Epicenter: Not located.

Depth: None computed.

Magnitude: None computed.

Five other earthquakes were felt at old Faithful following this one. They were felt at 13:16, 13:27, $13: 32$, 14:25, and $14: 27$ (R. A. Hutchinson, Park Geologist).

Intensity III: old Faithful.

15 September (G) Yellowstone National Park origin time: 134544.8
Epicenter:

Depth :

Magnitude:

$44.56 \mathrm{~N} ., 110.49 \mathrm{~W}$.

$5 \mathrm{~km}$

Intensity IV: Canyon Village, Fishing Bridqe, Lake.

2 October (G) Yellowstone National Park origin time: $\quad 135910.7$

Epicenter: $\quad 44.74 \mathrm{~N}, 110.78 \mathrm{~W}$.

Depth: $5 \mathrm{~km}$

Magnitude:

$3.5 \mathrm{ML}, 3.5 \mathrm{ML}(\mathrm{D})$

This earthquake and the one at 2355

42.6 are two of nine which were

felt in the park area on Octoher 2

in a 12-hour period from 1159 to

2355 UTC. The intensity data are from R. A. Hutchinson, Park Geologist (written commun., 1979).

Intensity III: Madison Junction. Intensity II: old Faithful.

2 October (G) Yellowstone National Park origin time: 235542.6

Epicenter: $\quad 44.71 \mathrm{~N}, 110.80 \mathrm{~W}$.

Depth:

Magnitude: $5 \mathrm{~km}$

Madison Junction, Norris.

Intensity III: Canyon Village.

Intensity II: old Faithful.

12 October Yellowstone National Park

Origin time: 1352

Epicenter: Not located.

Depth: None computed.

Magnitude: None computed.

This is the first of about 35 earthquakes occurring in a 90-minute period which were felt at Grant Village ( $R$. A. Hutchinson, written commun., 1979).

Intensity IV: Grant Village.

4 November (G) Hebgen Lake region origin time: $\quad 154943.8$

See Montana listing.

11 November (G) Yellowstone National Park Origin time: $\quad 204620.8$

Epicenter: $\quad 44.39 \mathrm{~N}, 110.25 \mathrm{~W}$.

Depth:

Magnitude:

$6 \mathrm{~km}$

Intensity II: Lake. 


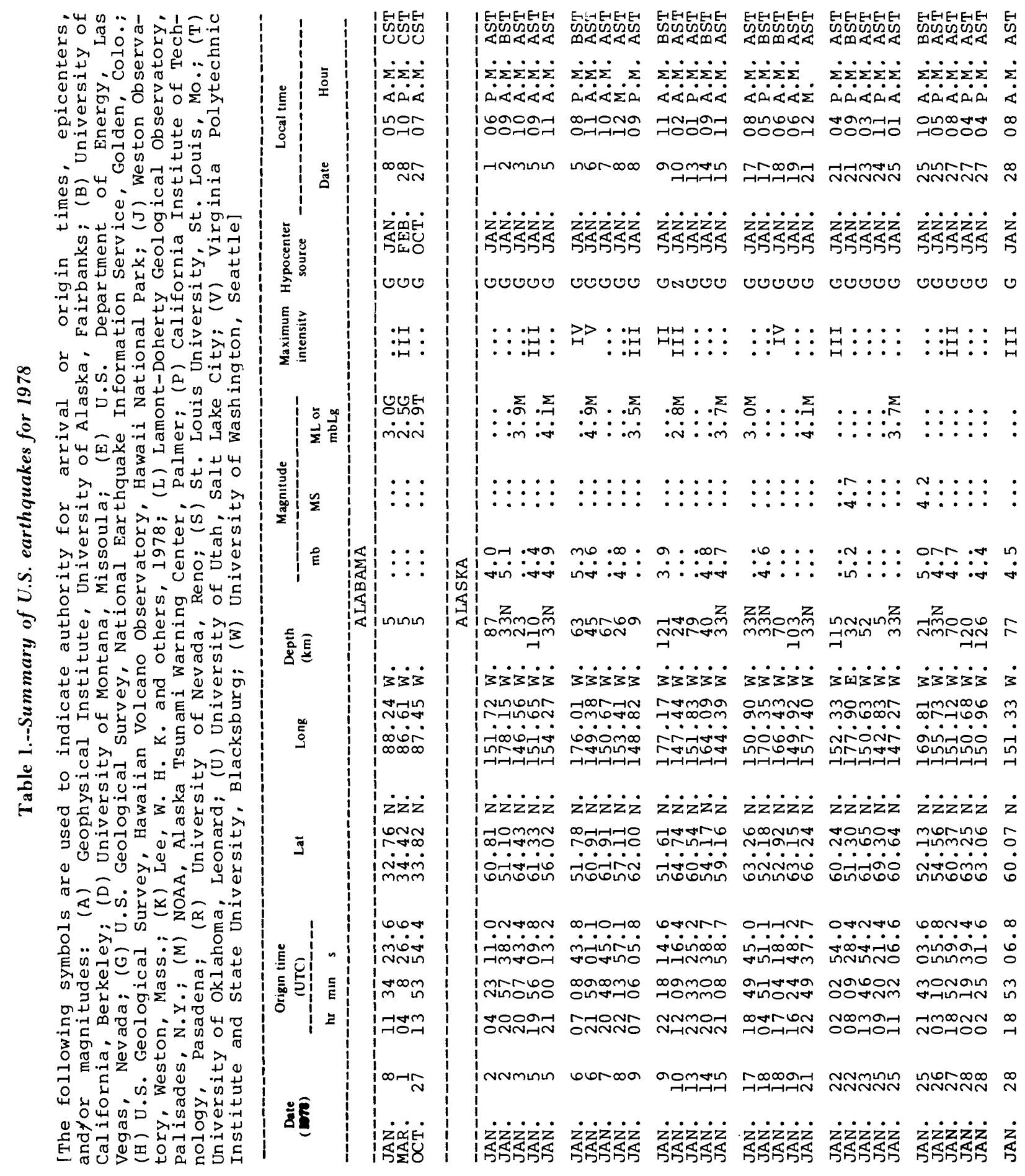




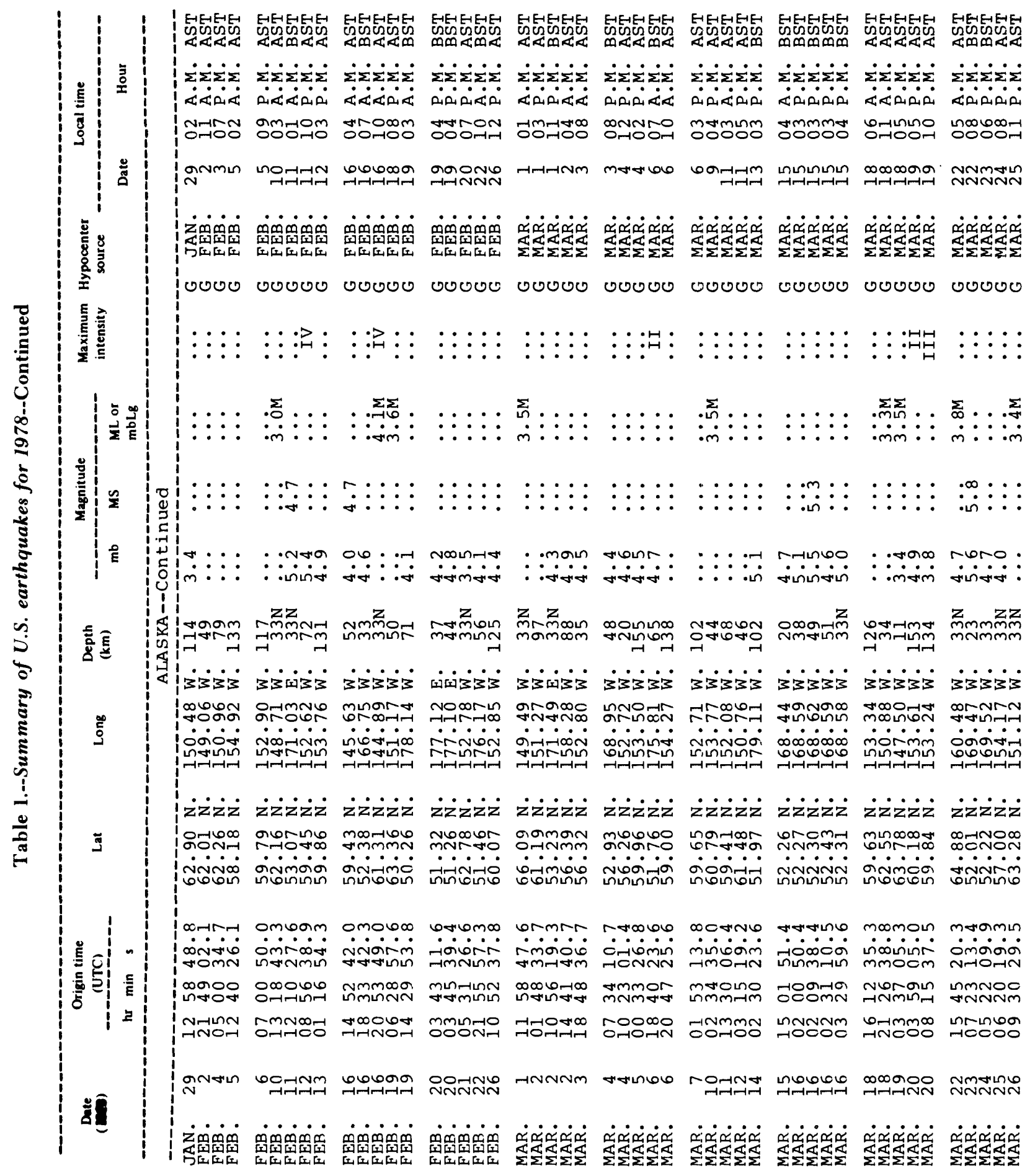




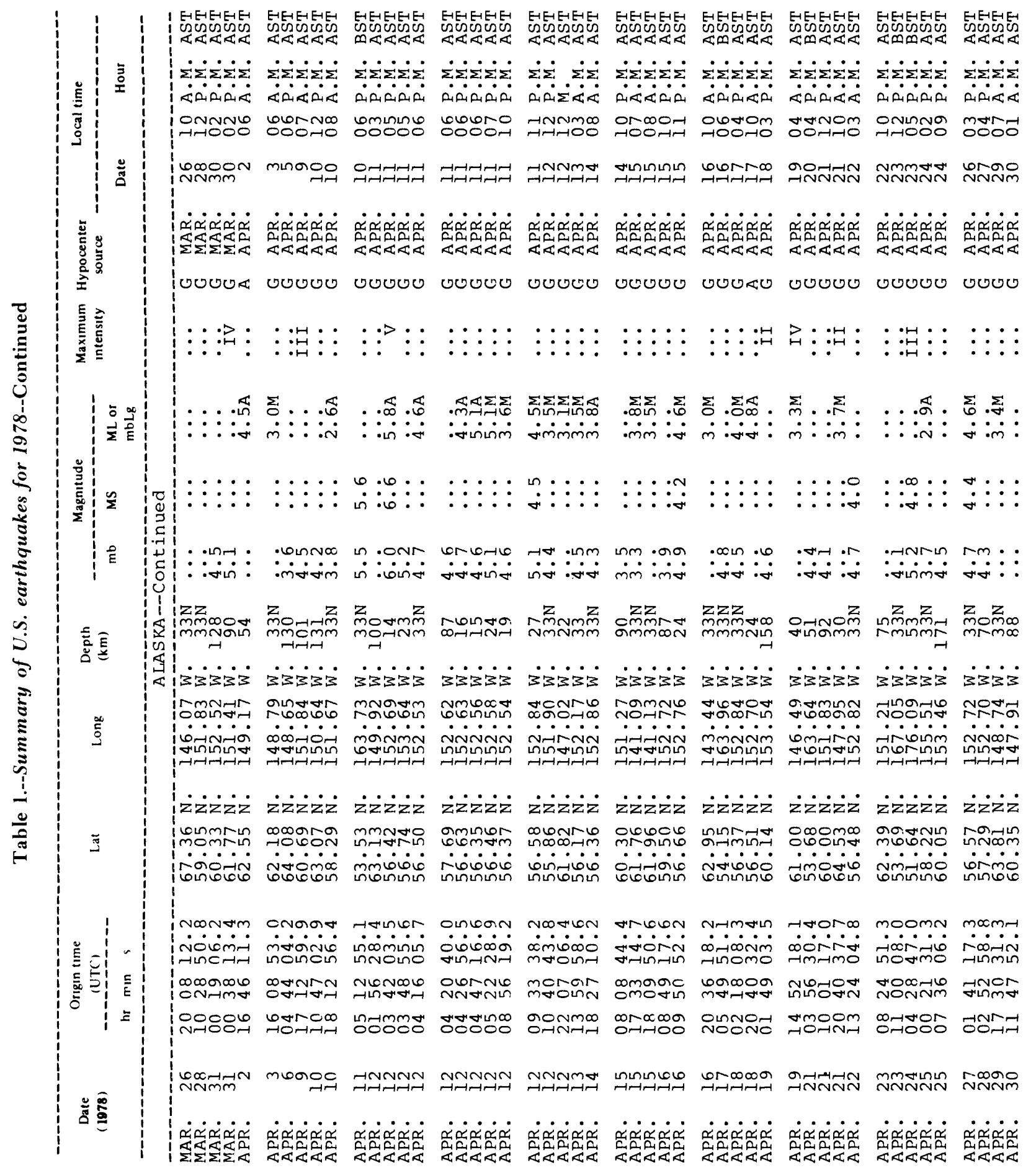




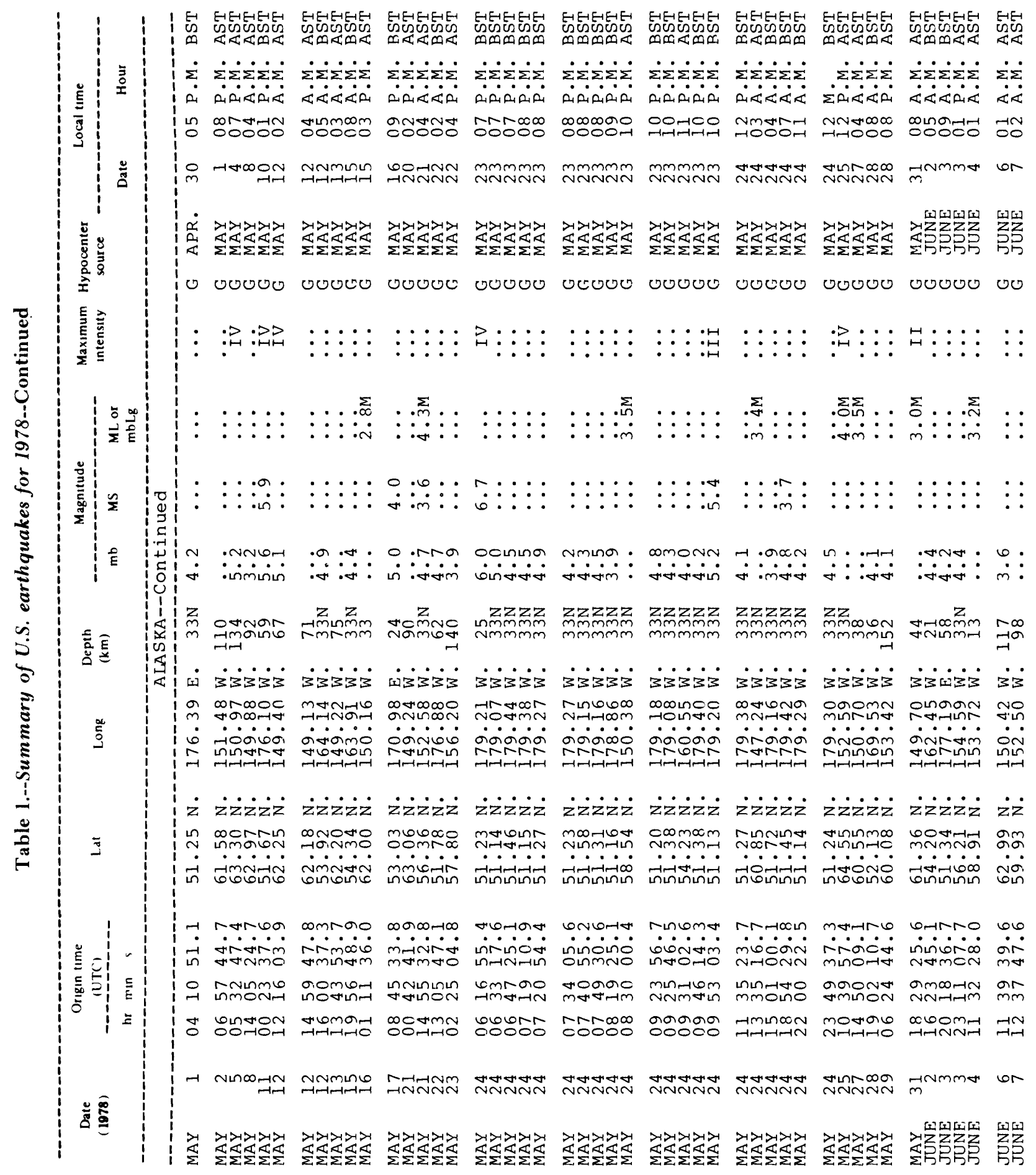




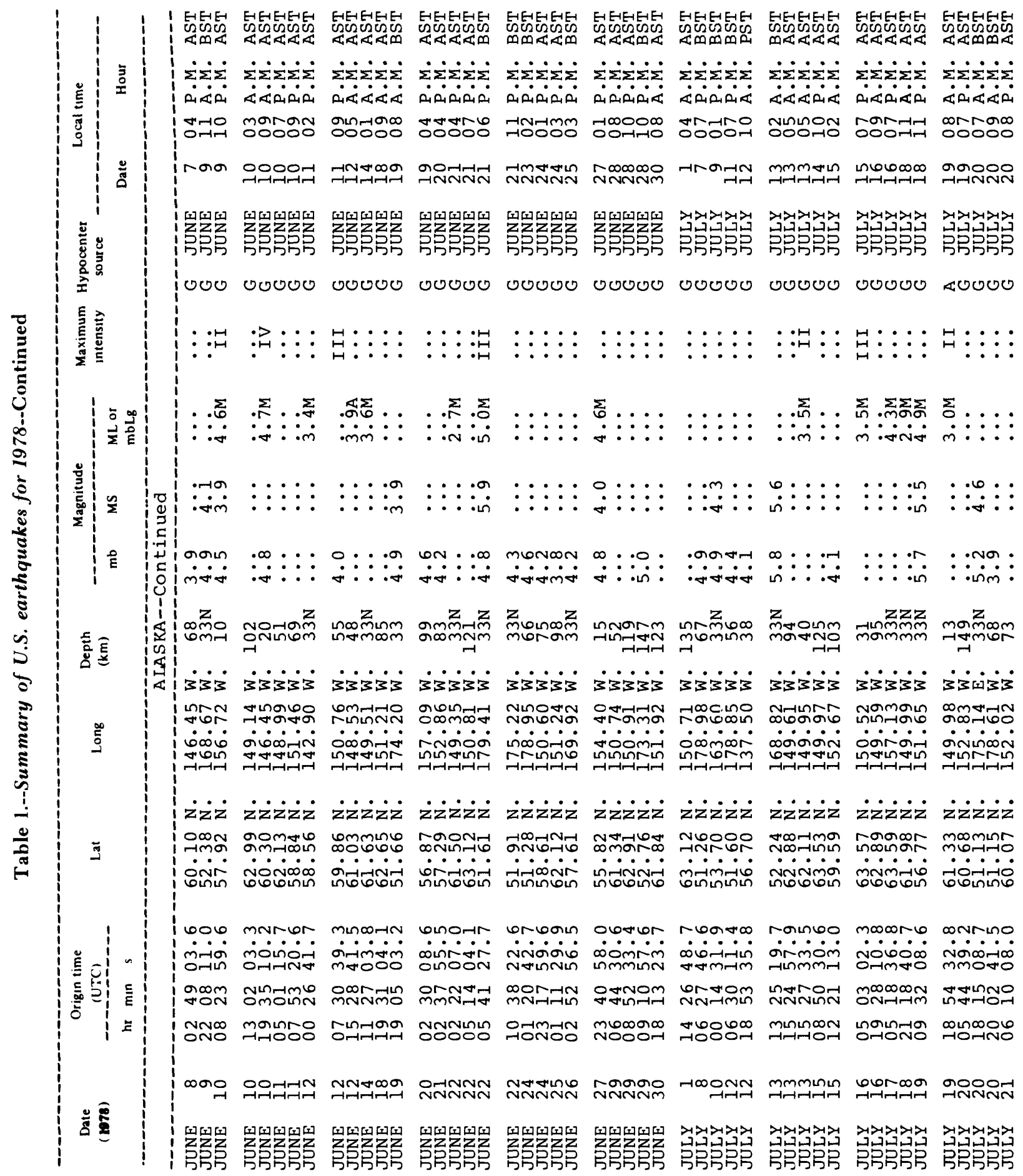




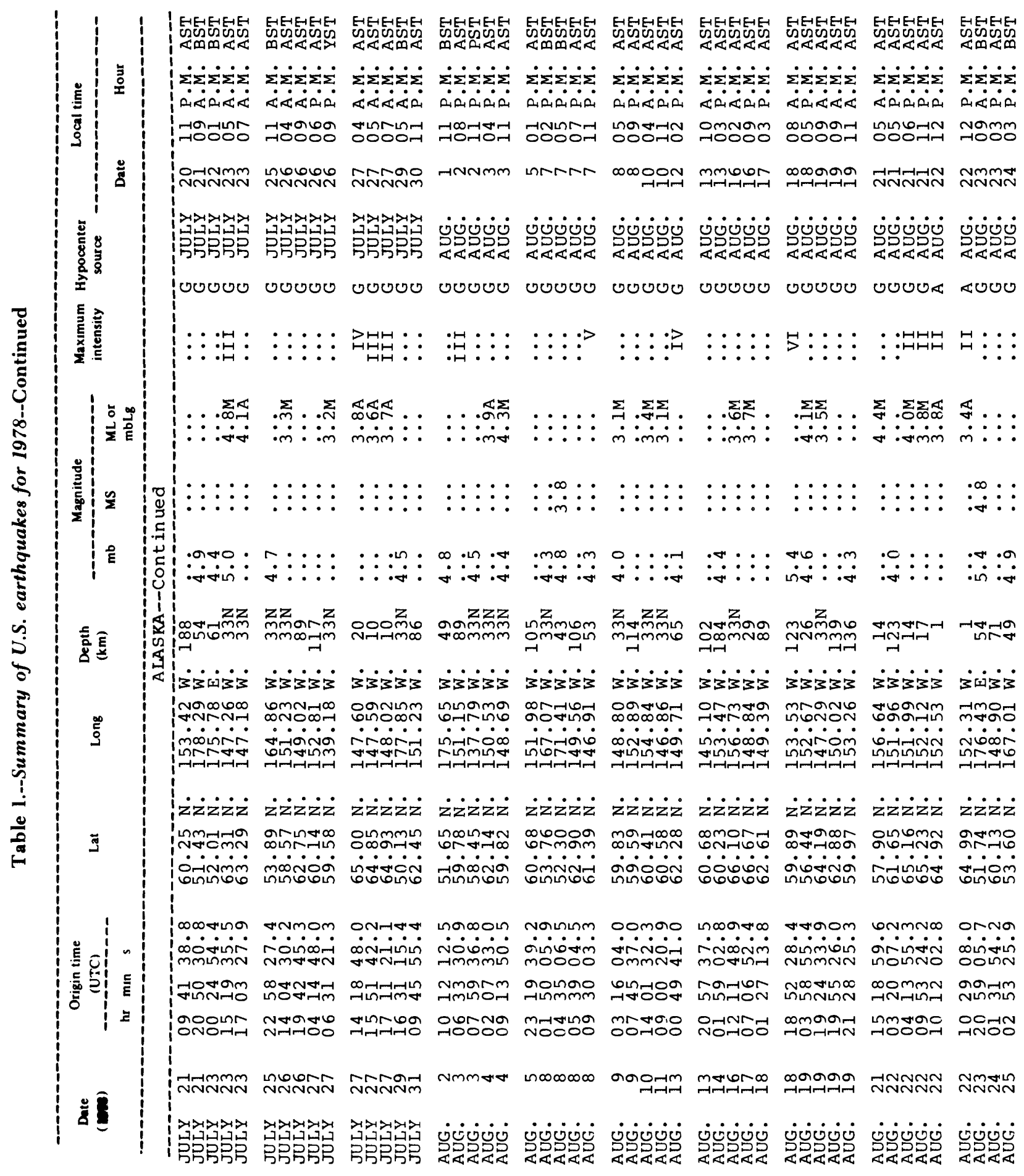




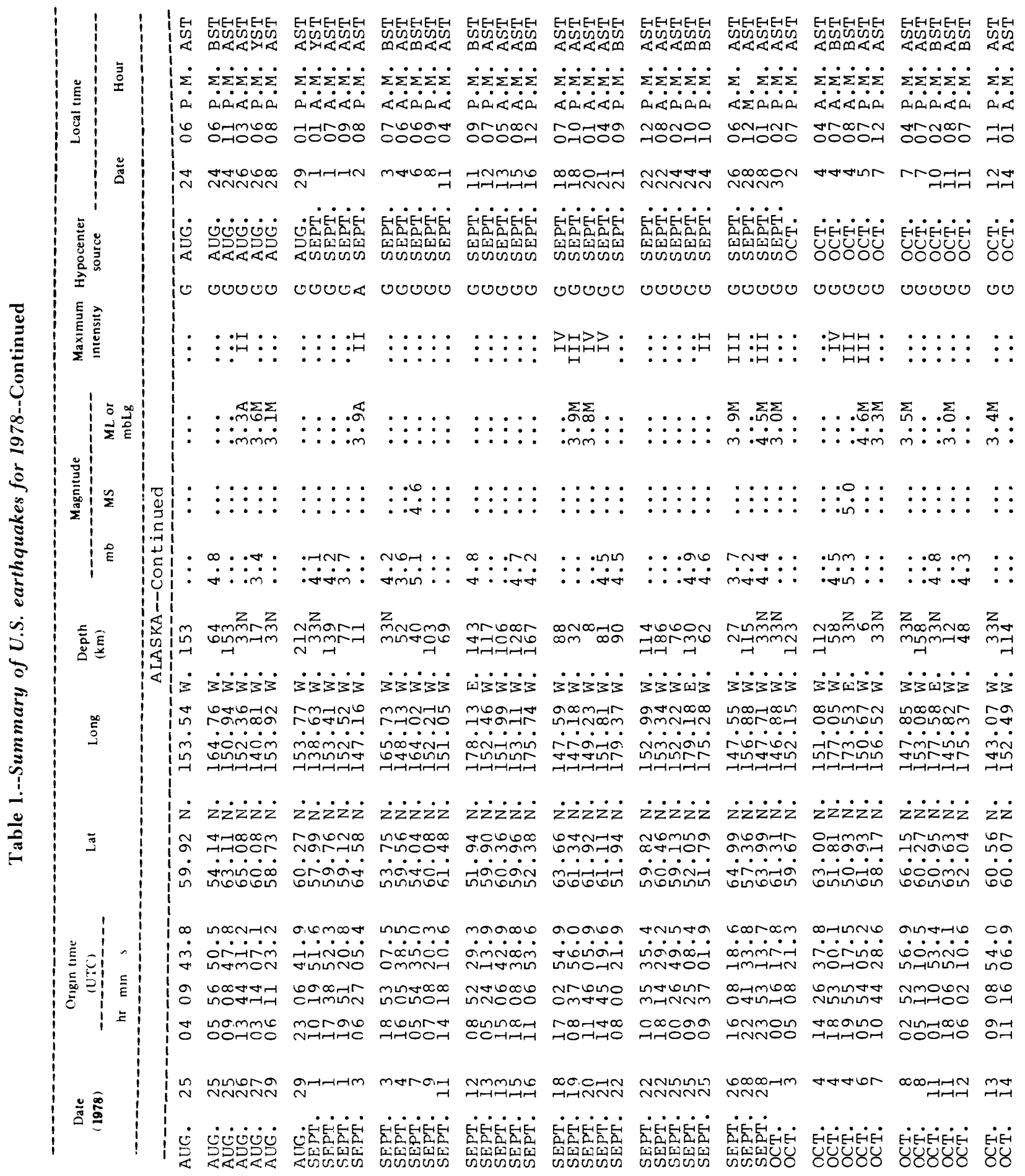




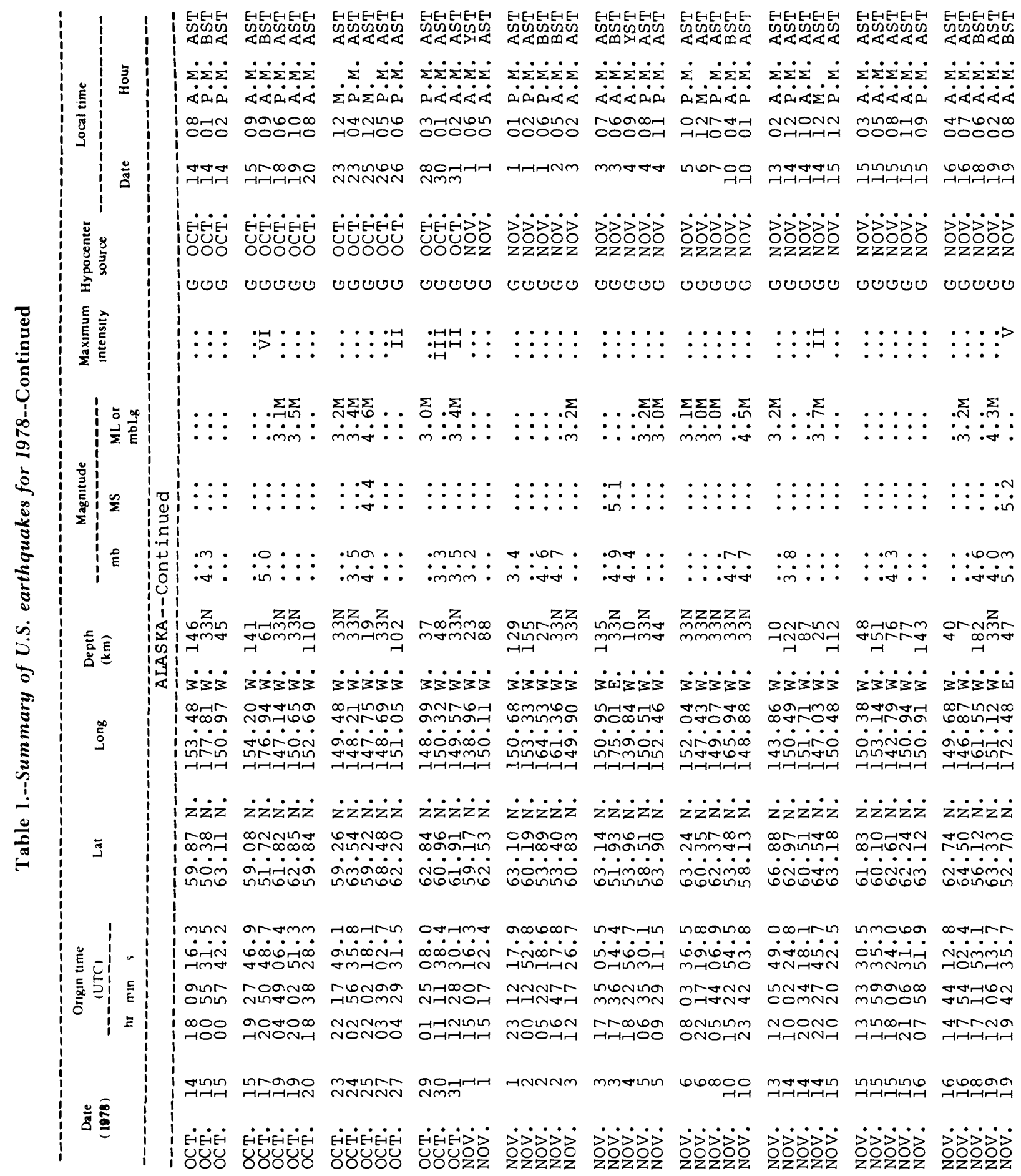




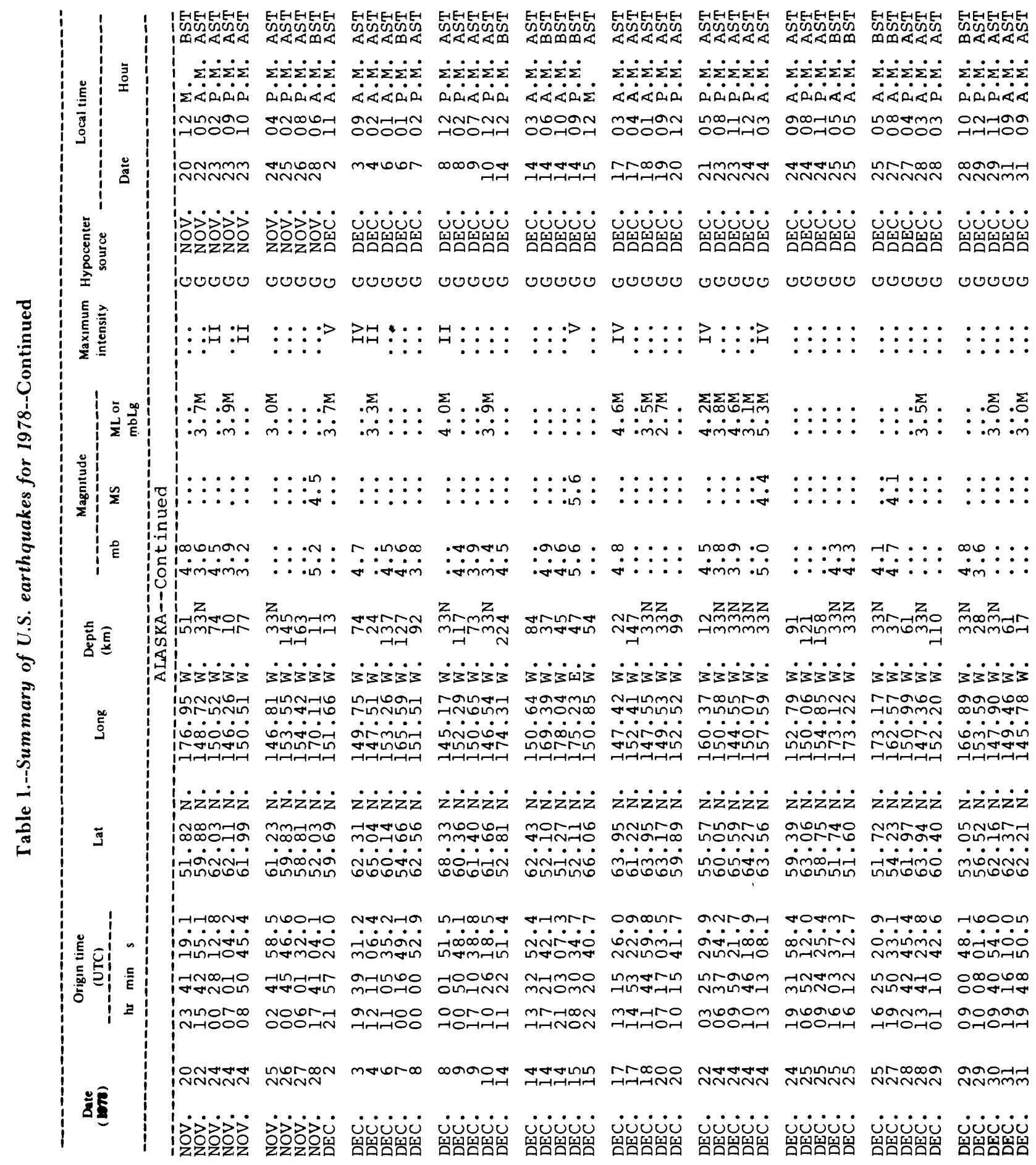




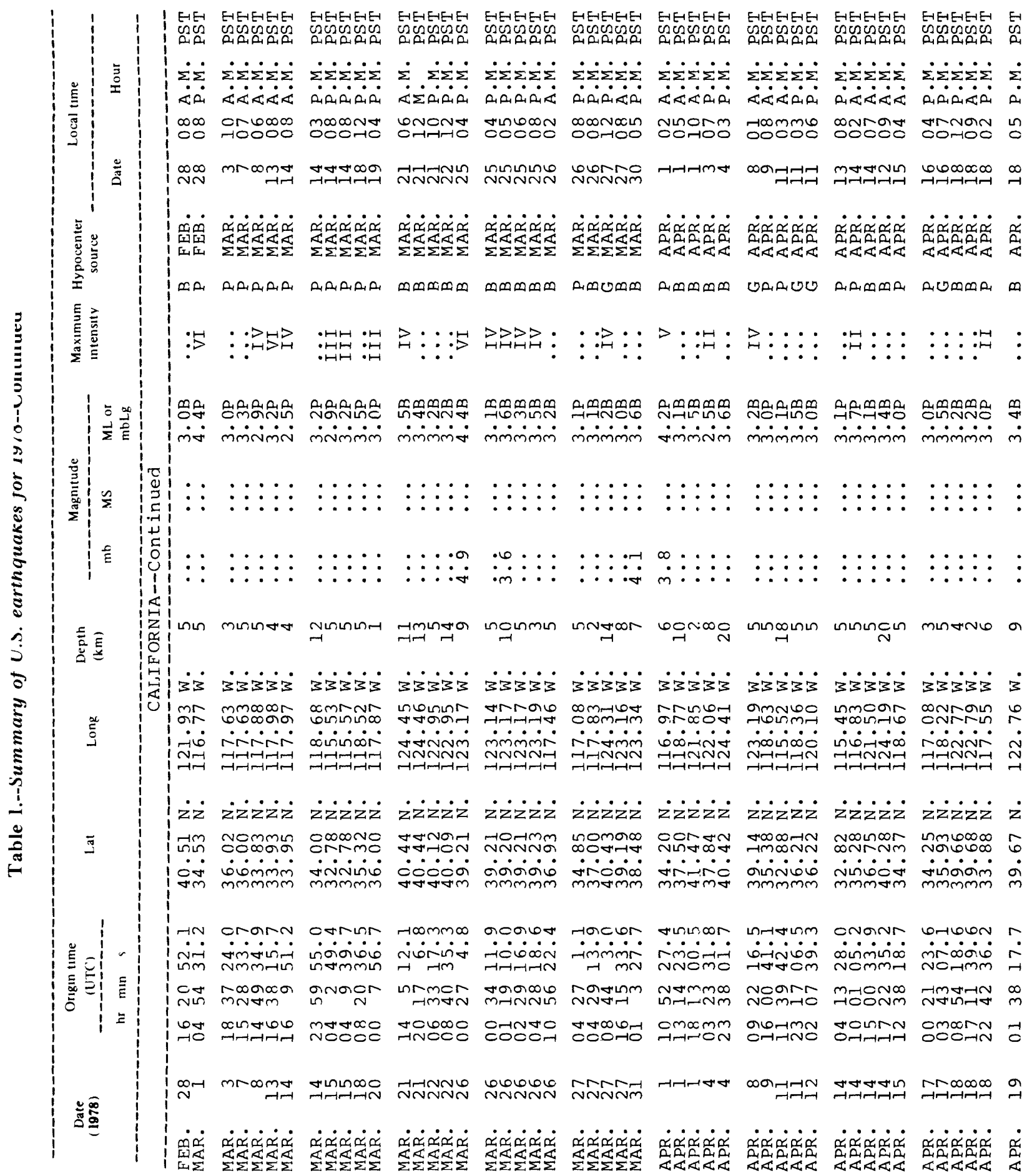




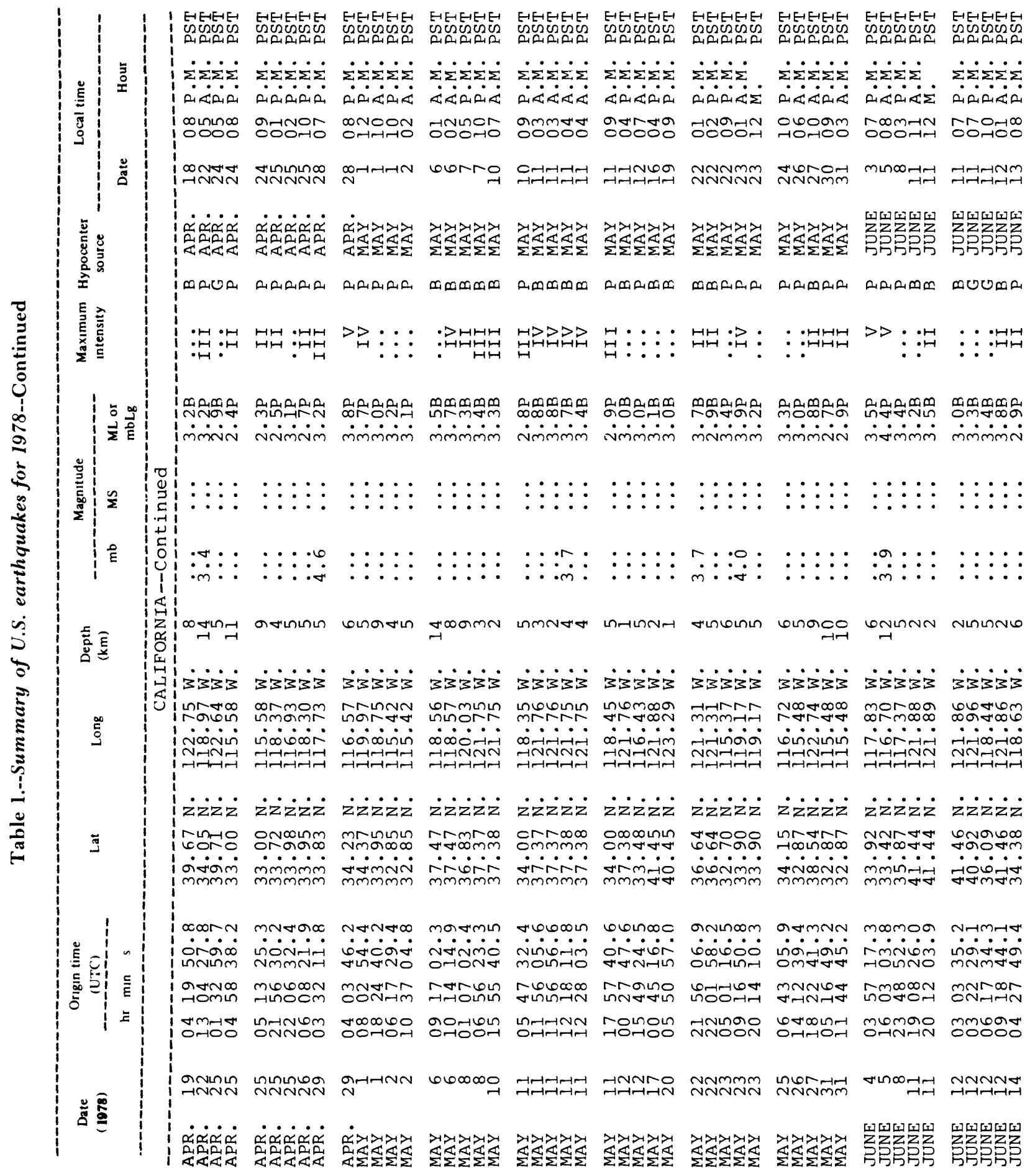




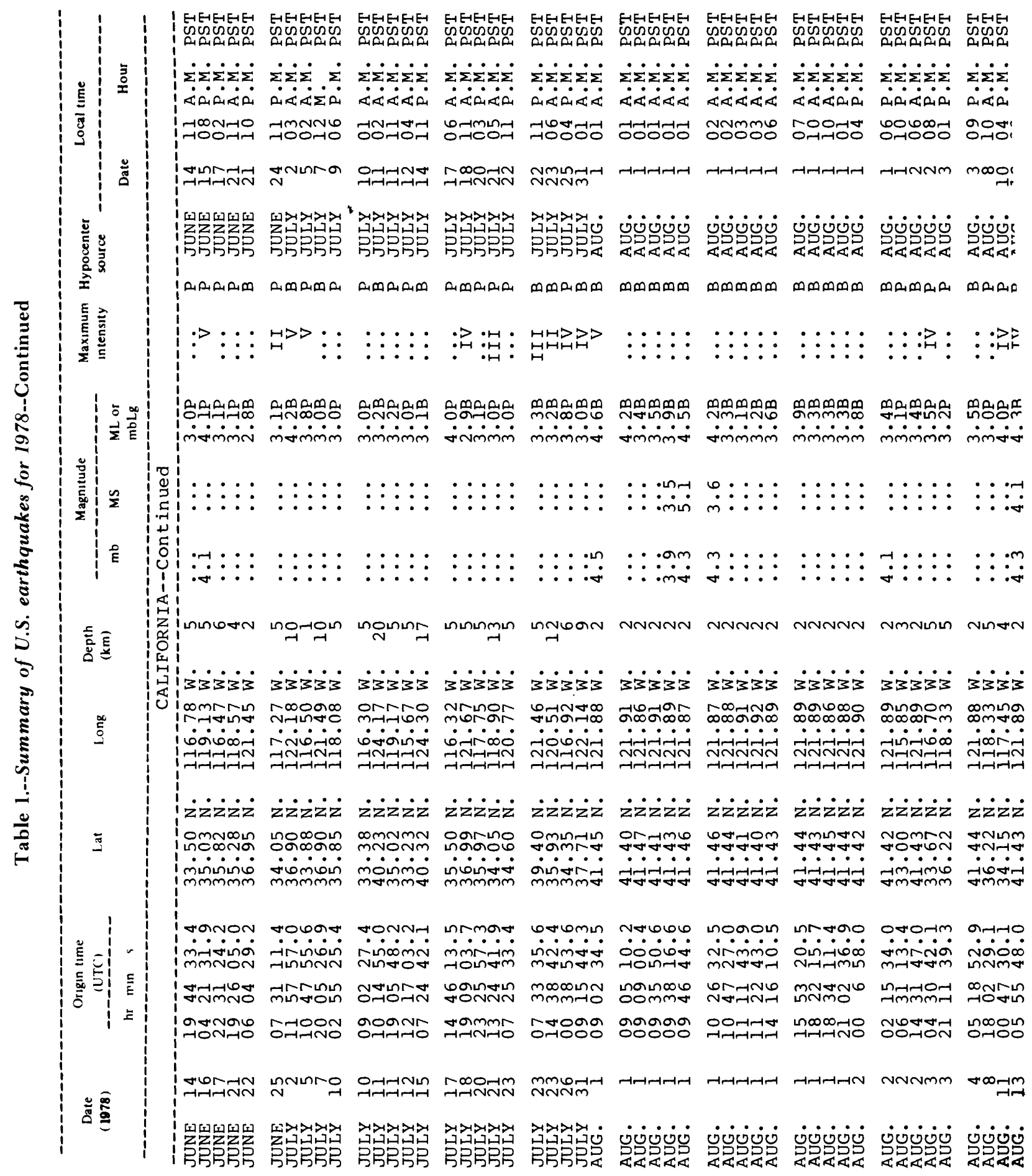




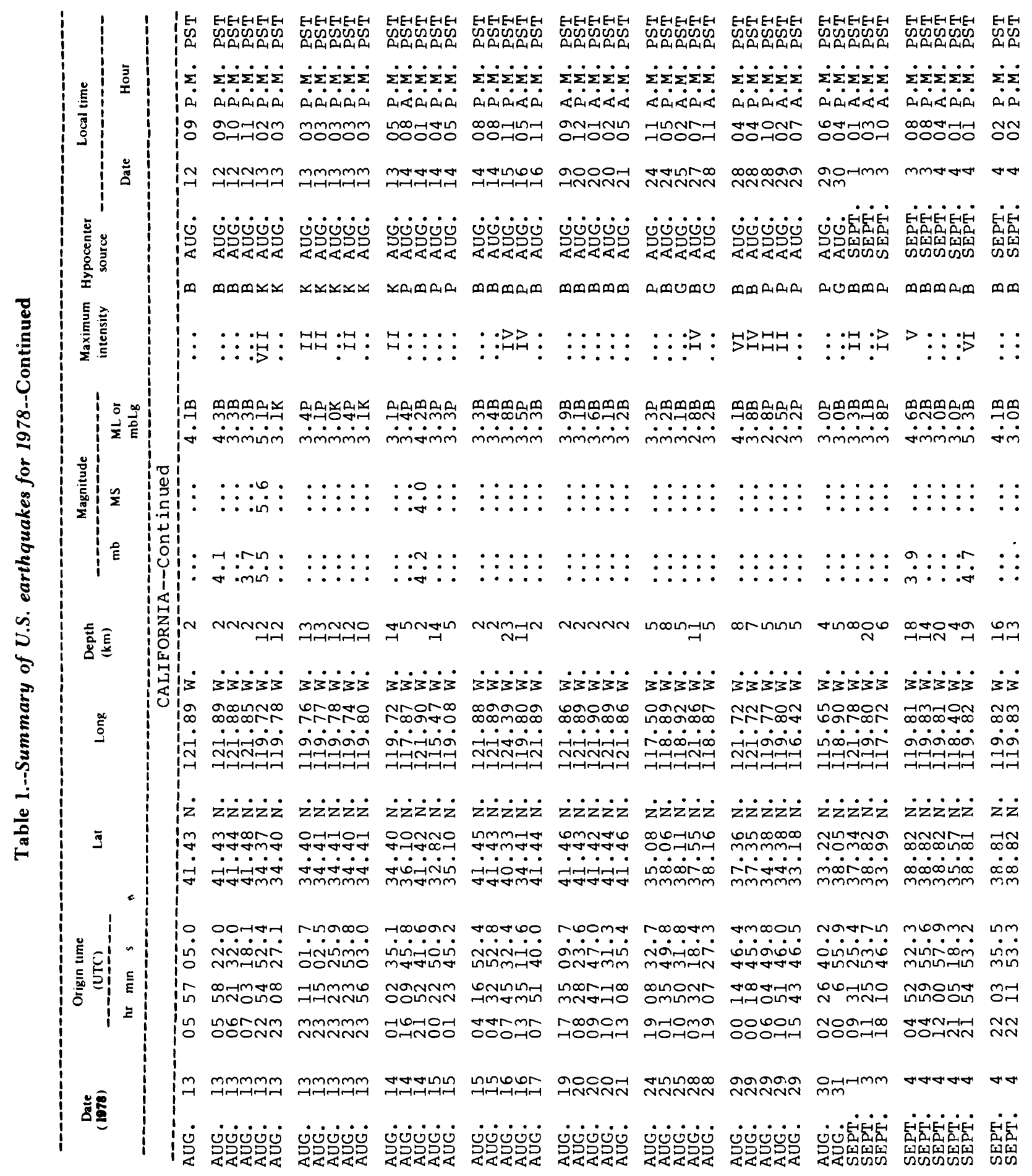




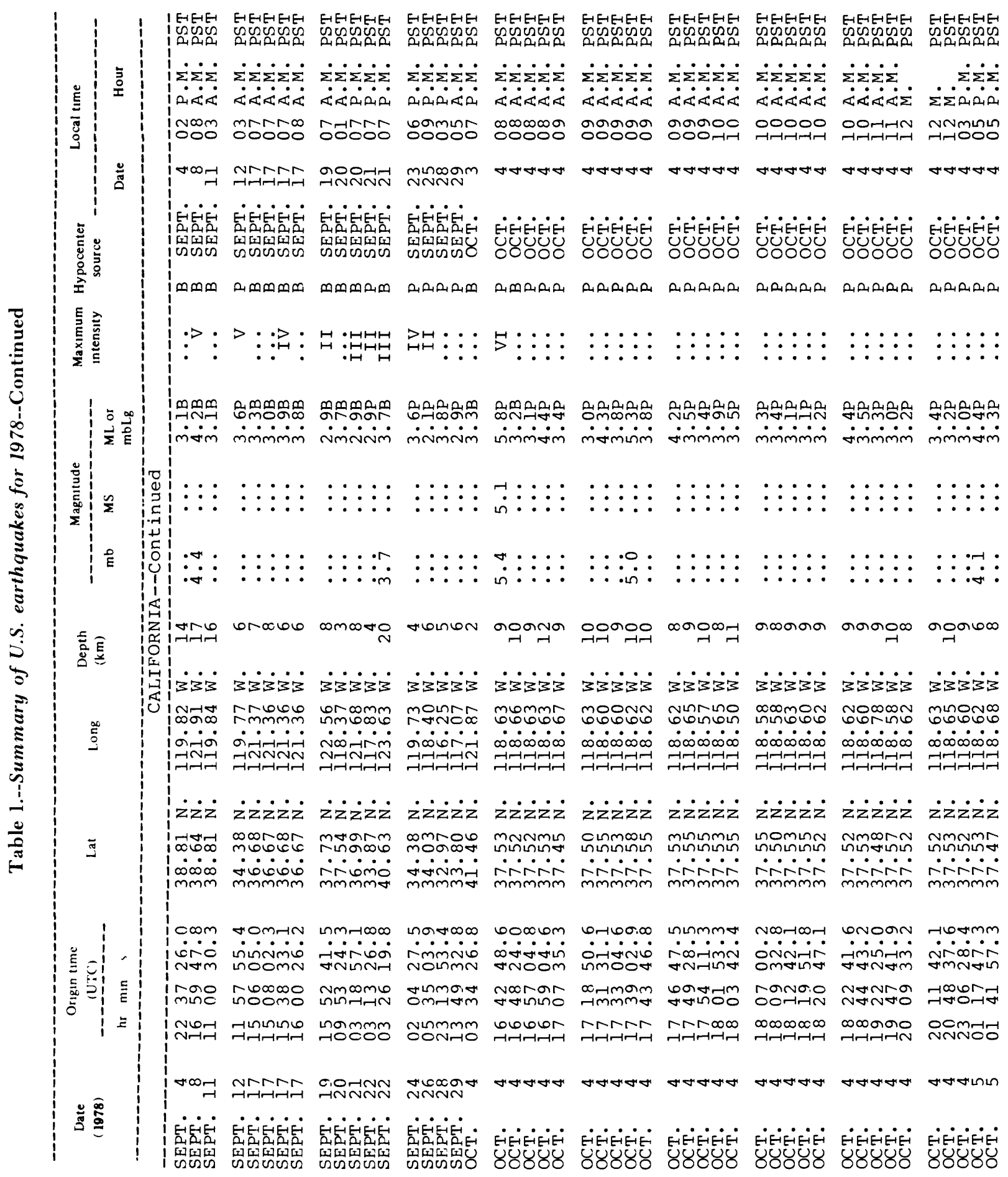




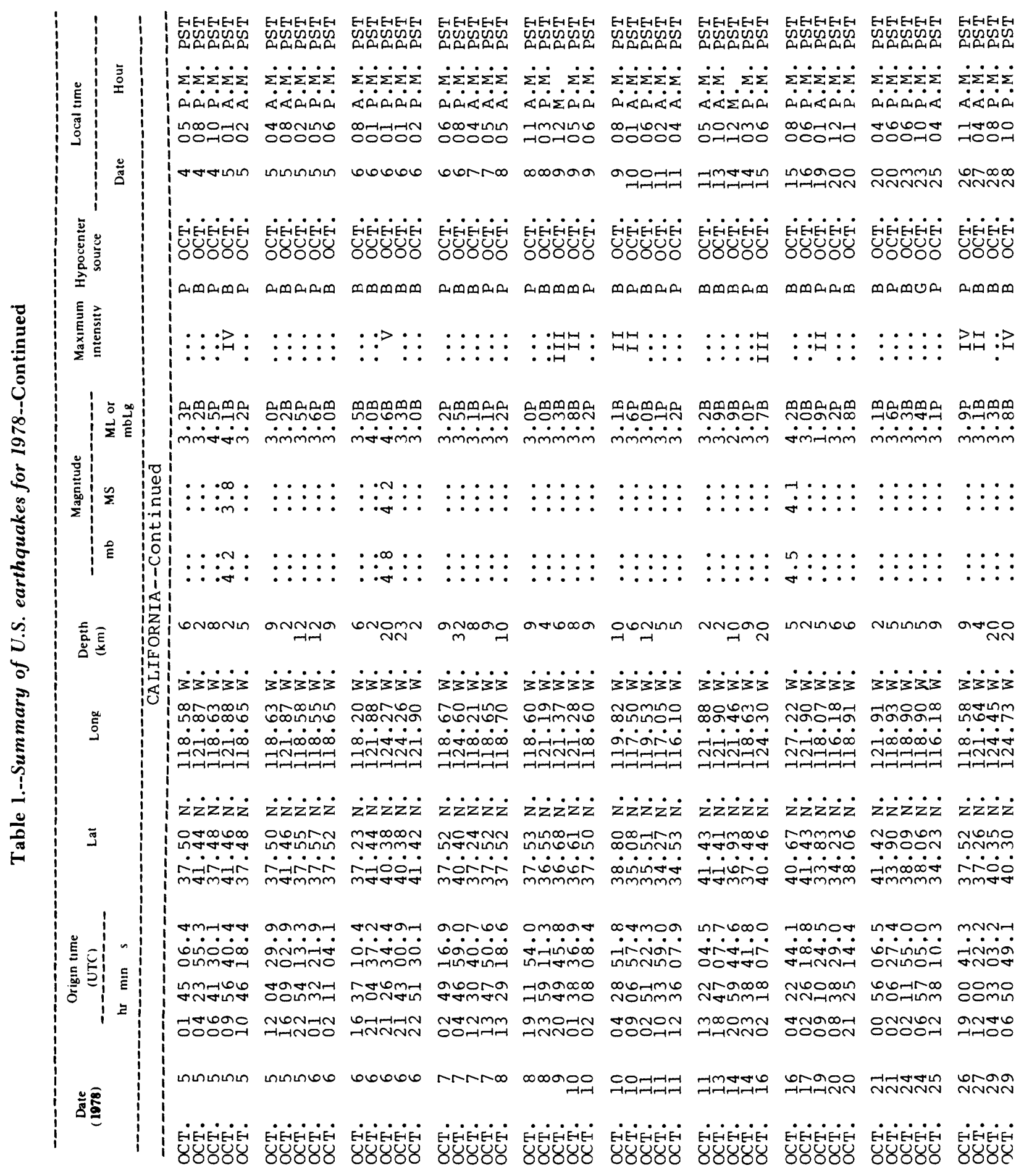




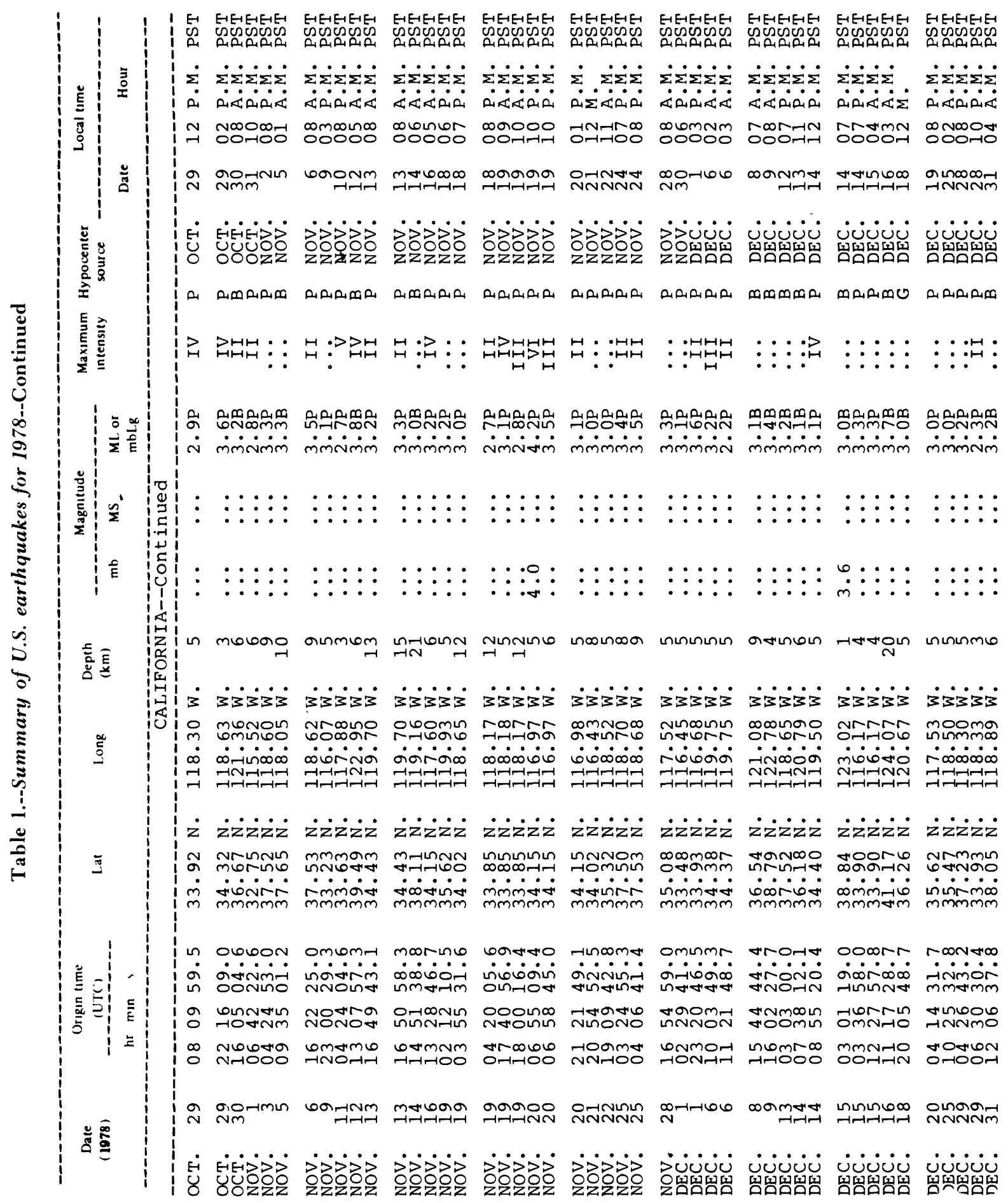




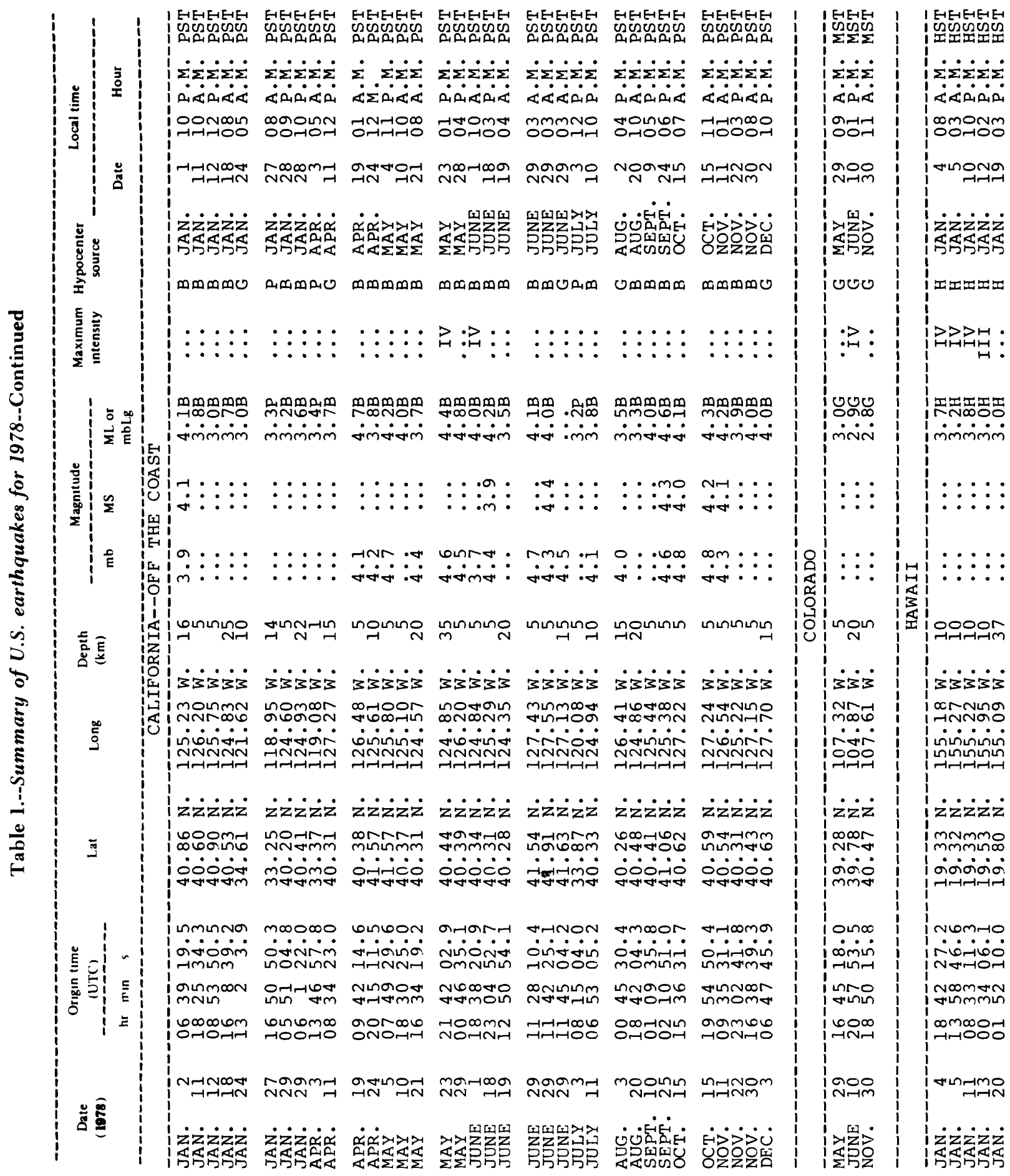




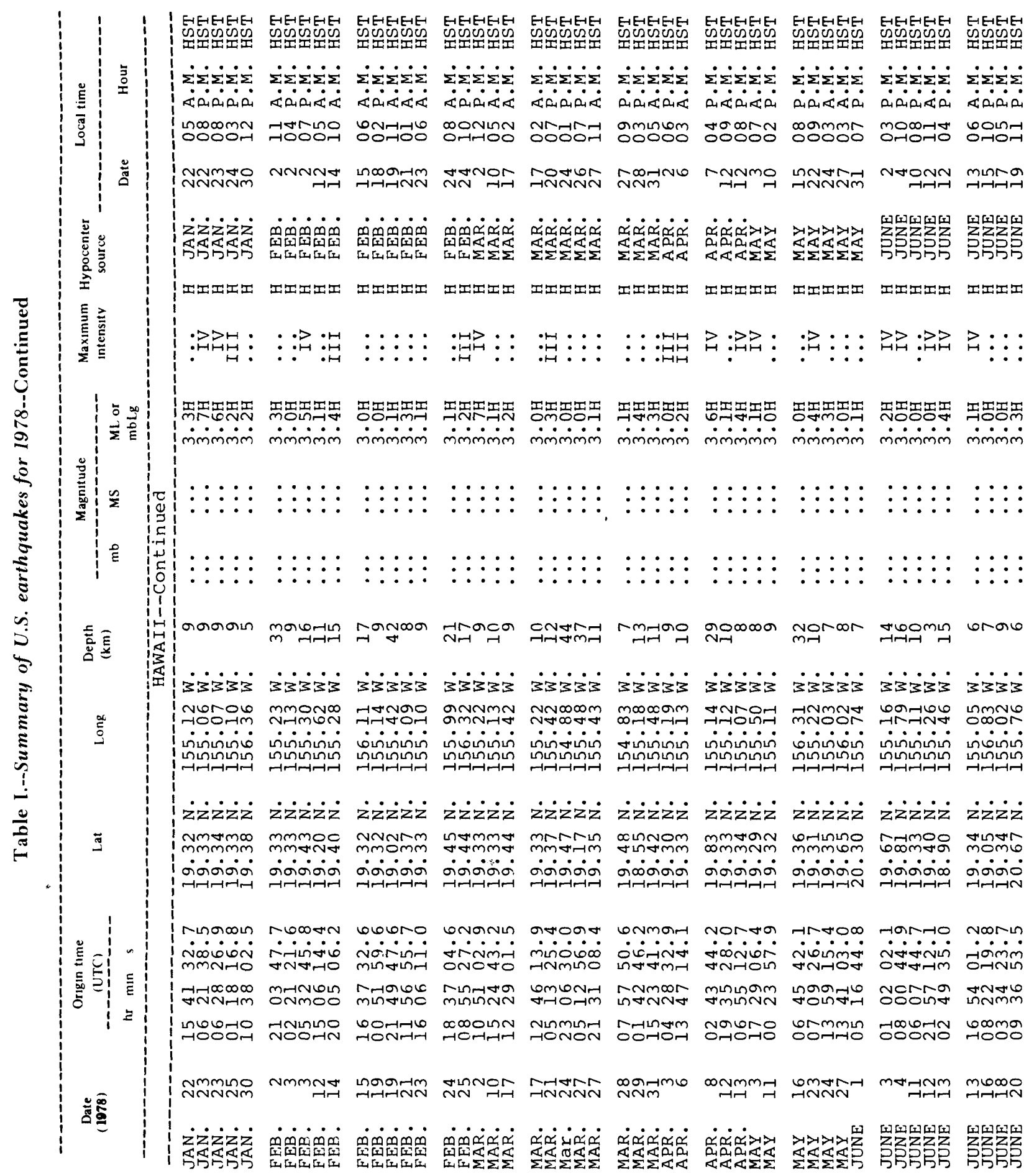




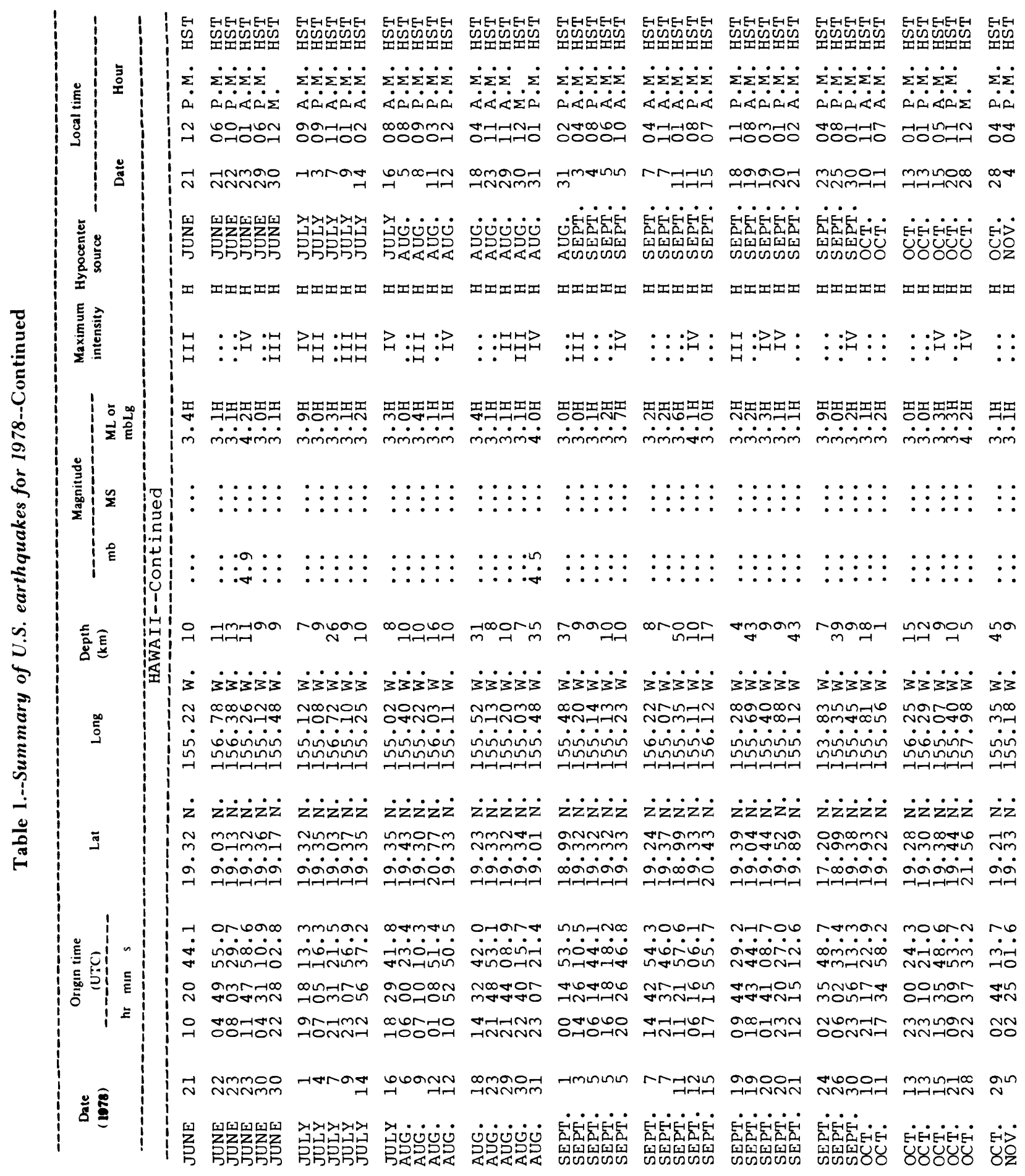



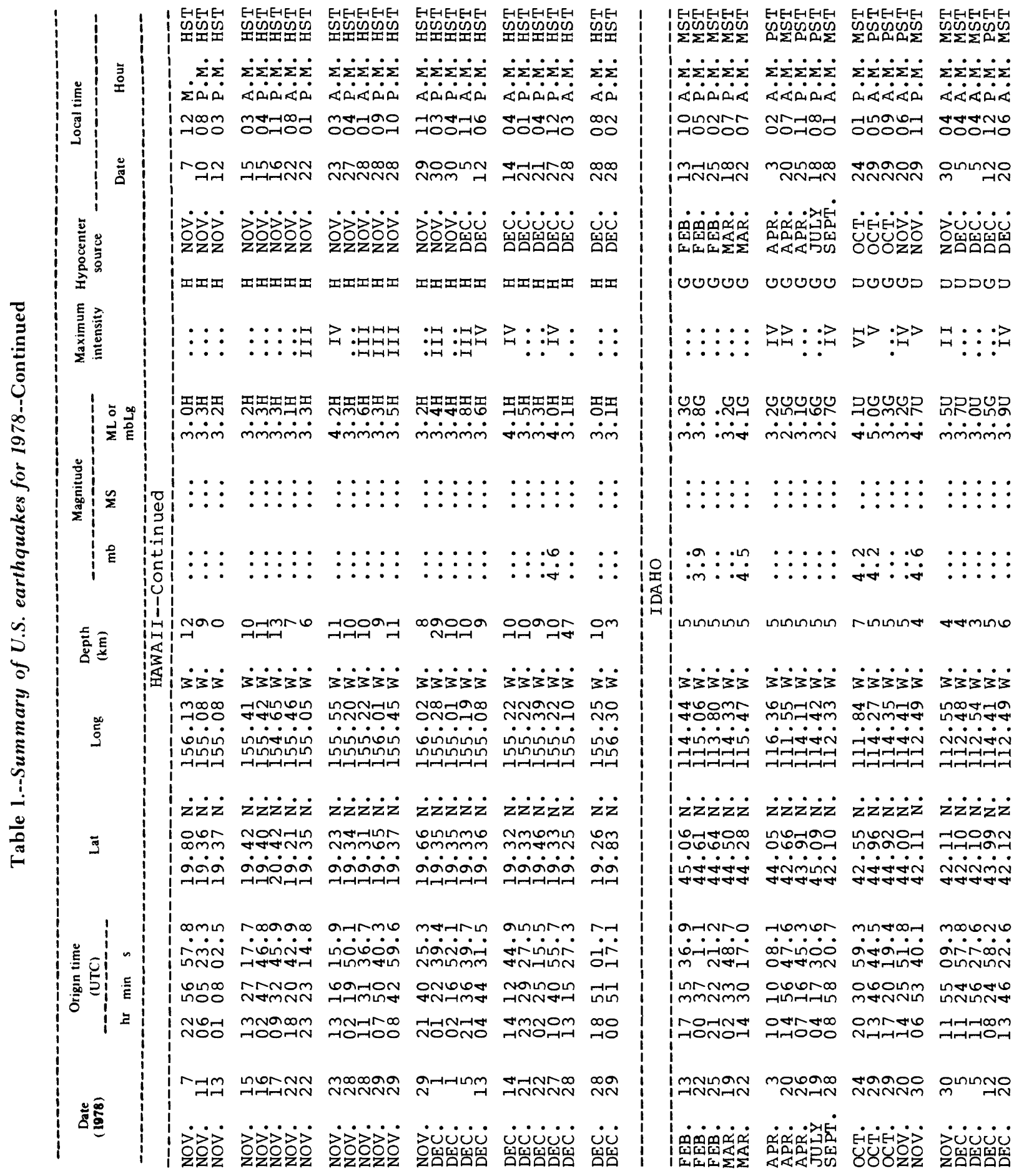


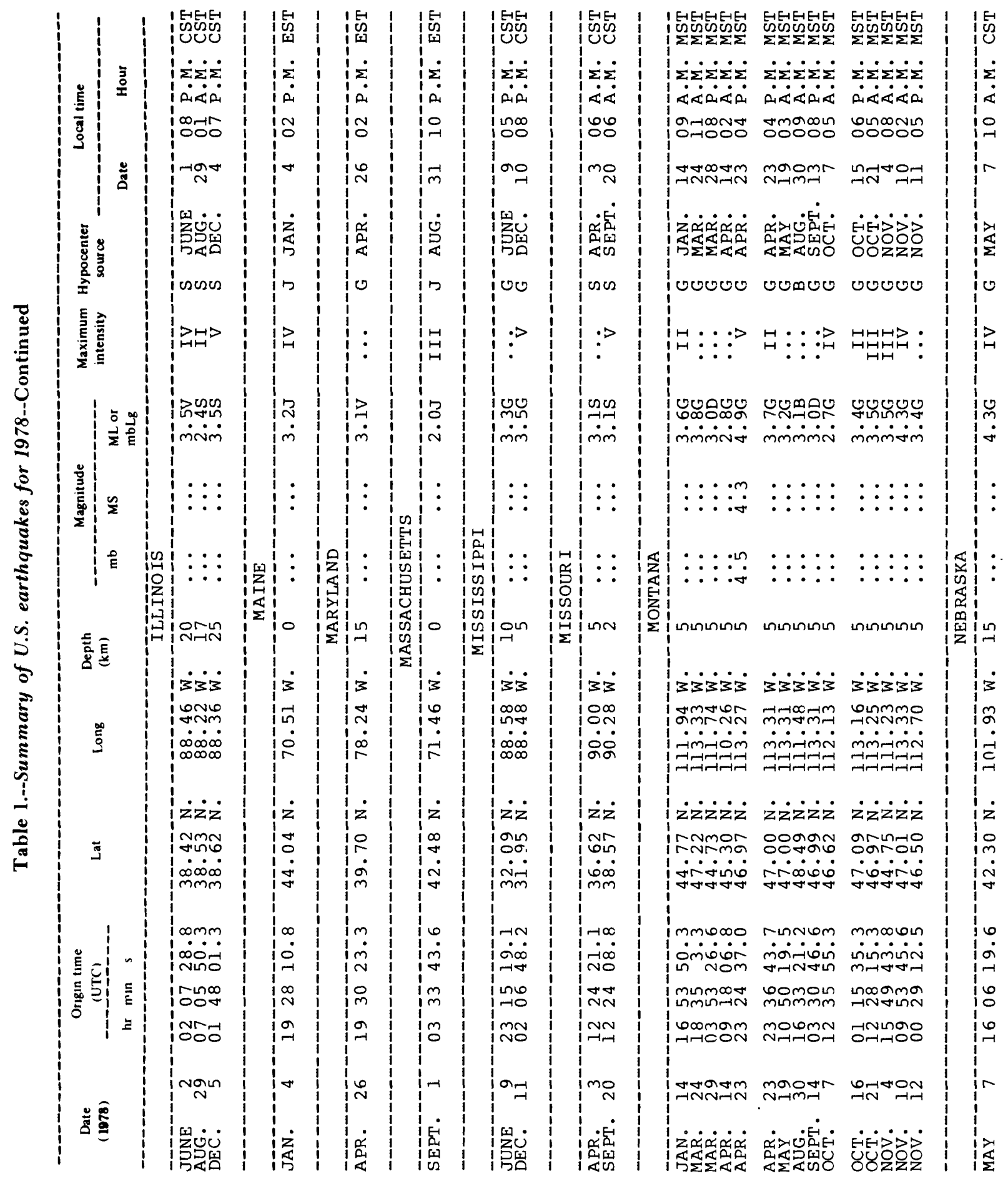




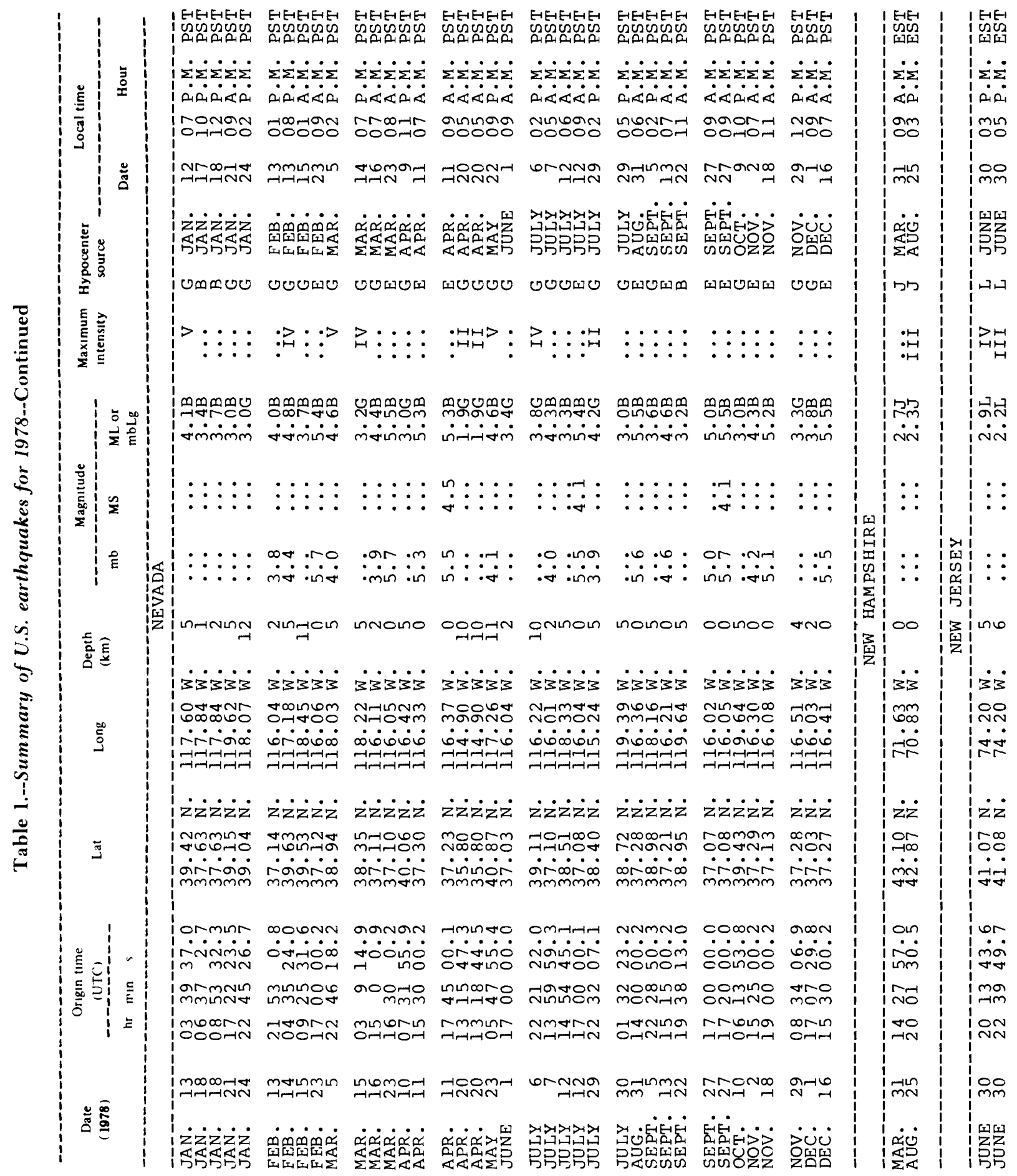




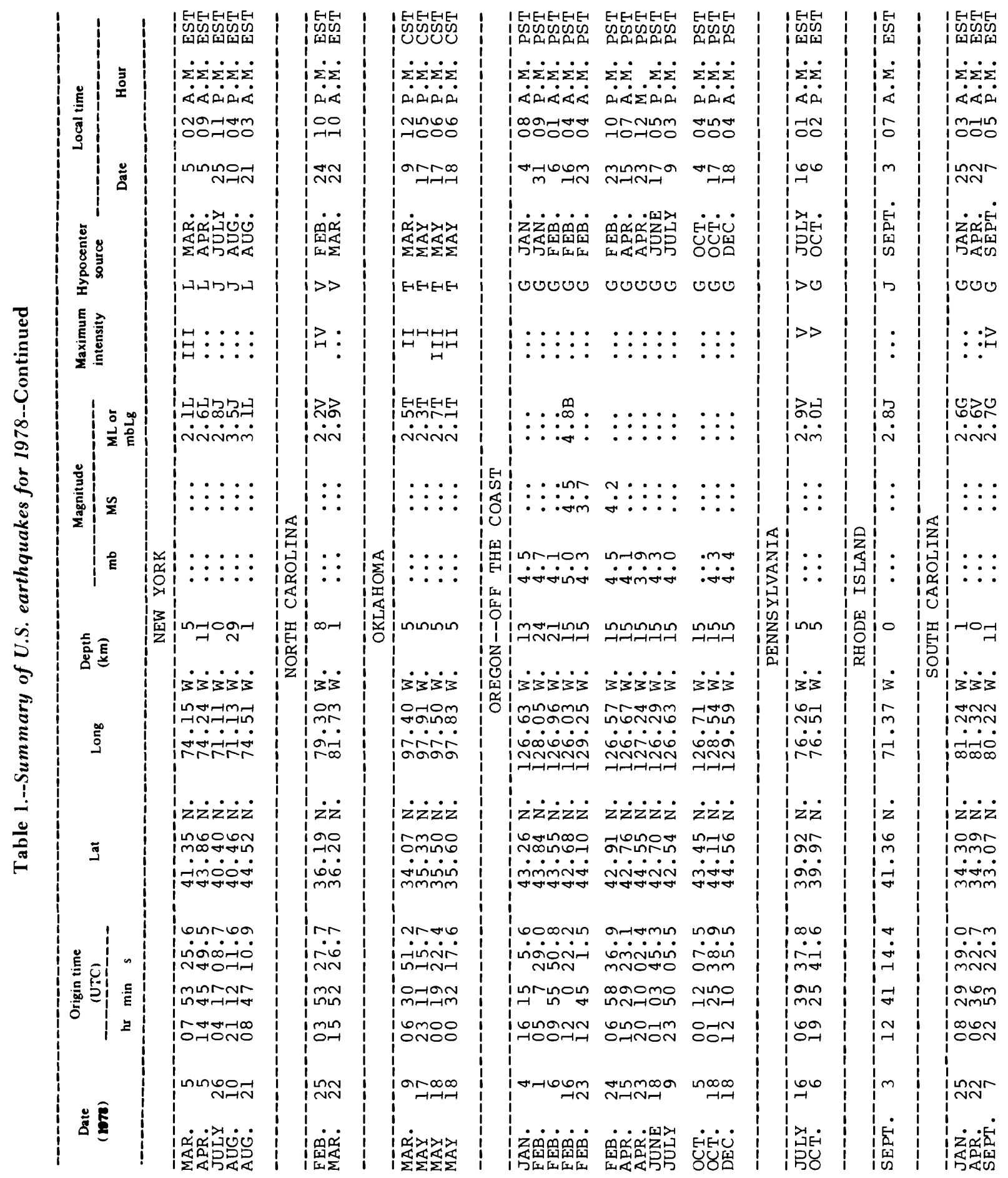




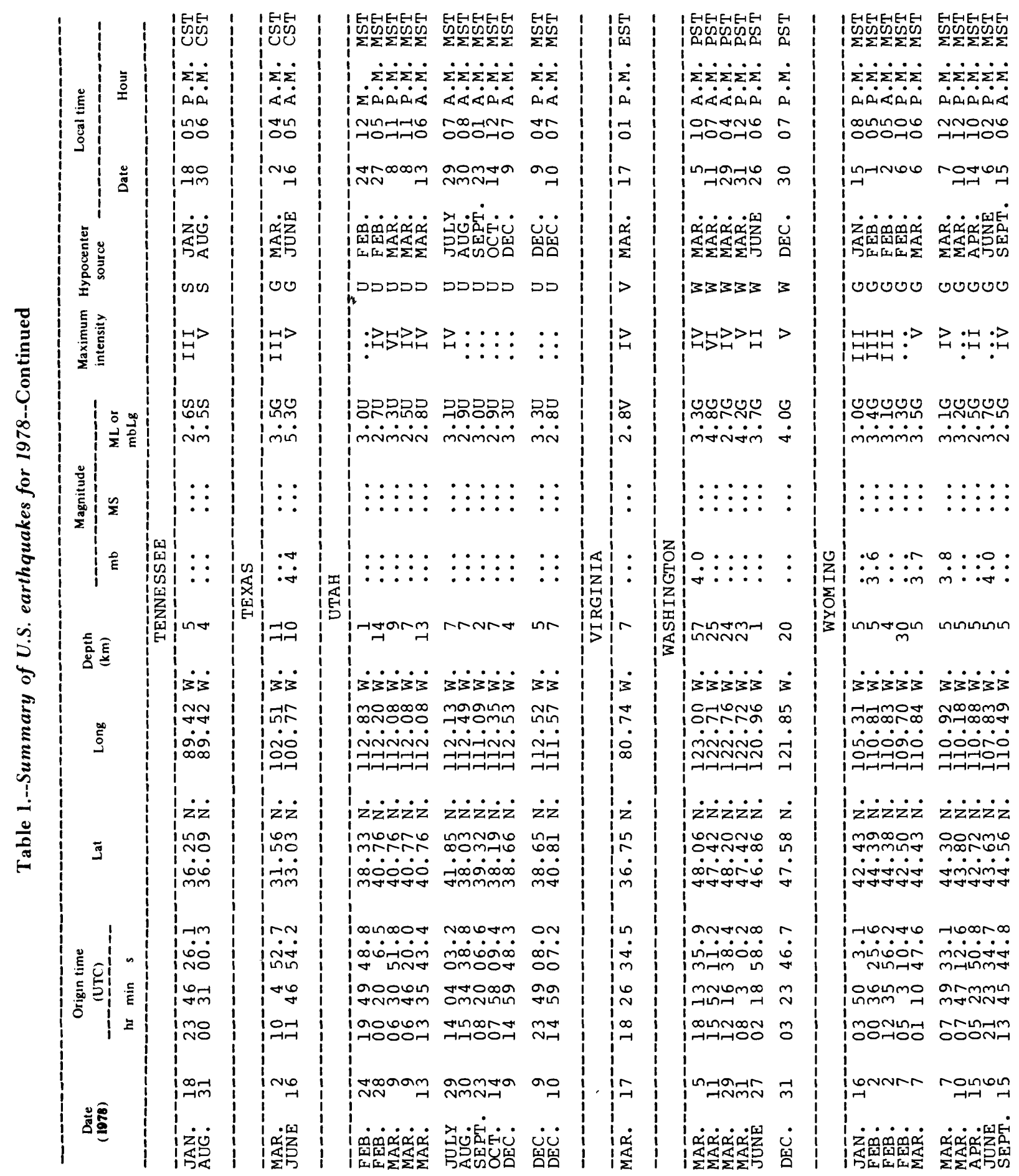




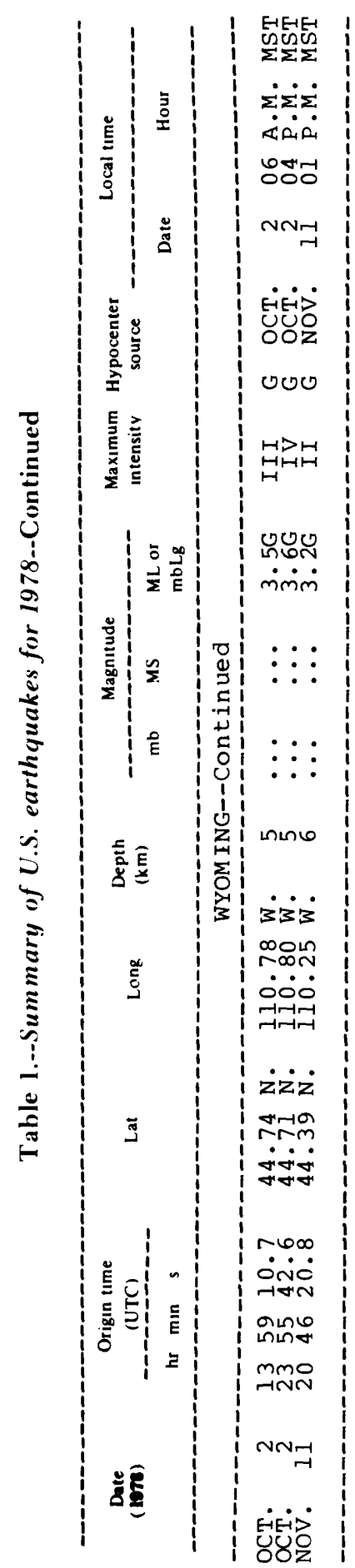




\section{Network Operations}

\section{ALASKA EARTHQUAKES, 1978}

\author{
By Larry Gedney \\ Geophysical Institute \\ University of Alaska \\ Fairbanks, Alaska 99701
}

During 1978, the Geophysical Institute of the University of Alaska located 4,281 Alaskan earthquakes occurring within the boundaries of its existing Alaskan seismographic network. The results were presented in the series of publications, "Summary of Alaskan Earthquakes," prepared quarterly by the Geophysical Institute. Magnitudes of the individual earthquakes ranged from below 0 (in areas which are heavily instrumented, such as the Fairbanks seismic zone) to magnitude 6.5 . That largest event occurred southeast of Kodiak Island on April 12, 1978. The Institute's seismographic coverage does not permit the location of earthquakes in either southeast Alaska or the Aleutians.

Owing to various research and monitoring needs, there exists several identifiable subnets of stations in the Geophysical Institute's seismographic coverage. A 6-station subnet was operated in northeastern Alaska to evaluate seismic hazards in the Brooks Range and adjacent areas where petroleum development and production activities are underway or planned. A subnet around the Seward Peninsula was established to evaluate hazards in an area planned for future hydrocarbon development in Norton and Kotzebue Sounds. A subnet in southern Alaska, around Cook Inlet and Kodiak Island, was also designed to provide information of seismic hazards in an area soon to be undergoing hydrocarbon development, as well as for monitoring a seismic gap, expected to undergo a major earthquake in the near future. These three subnets have been largely supported by funds from the Outer Continental Shelf Environmental Assessment Program, administered by the National oceanographic and Atmospheric Administration, and the southern subnet has also received support from the U.S. Department of Energy. A subnet in central Alaska has been supported largely by funds from the state of Alaska. This subnet occupies the most populated area of the state and most of the transportation corridors.

Data originating at the various seismometers were transmitted in analog form to central recording sites at Fairbanks and Homer, Alaska, via combinations of VHF, tropospheric scatter and satellite radio 1 inks. Communication ties with the
NOAA Alaska Tsunami Warning Center at Pa1mer, Alaska, permit real-time mutual data exchange with both the USGS and NOAA. cooperative data acquisition and analysis also is performed with the Lamont Geophysical observatory which operated seismic stations in the Shumagin region of the Aleutians.

\section{NORTHERN AND CENTRAL CALIFORNIA EARTHQUAKES, 1978}

By Robert A. Uhrhammer Se ismographic Station 475 Earth Sciences Building University of California, Berkeley Berkeley, California 94720

Some 5,200 events were noted on summary sheets and 1,220 teleseisms and 440 local earthquakes were analyzed during 1978. The Bulletin of the Seismographic Stations, Volume 48, Nos. I and 2 (McKenzie and others, 1978), contains location and magnitude information for 210 earthquakes $(3.0 \leq M L \leq 5.8)$ located in northern and central california and adjoining regions. The epicentral locations are plotted in figure 26 (the numbers correspond to a 1 ist in the bulletin).

A plot of the cumulative number of earthquakes versus local Richter magnitude (ML) is given in figure 27. The data set consists of 895 earthquakes $(3.0<M L<5.9)$ listed in the U. C. Bulletin of the Seismographic Stations, in a $280,000 \mathrm{sq} \mathrm{km}$ region in northern and central California during the decade of January l, 1967 to December 31 , 1976. The region is bounded on the north and east by the california border, on the southeast by the dashed $l$ ine in figure 26, on the southwest by a 1 ine connecting $35^{\circ} \mathrm{N} .-121^{\circ} \mathrm{W}$. and $39^{\circ}$ N. - $125^{\circ} \mathrm{W}$. , and on the west by $125^{\circ} \mathrm{W}$. longitude. The earthquakes are grouped into 20 consecutive 6-month intervals for analysis and the average cumulative number of earthquakes (N) (total number a magnitude $>M L)$ in a 6 -month interval is given by

$$
\log (N)=4.412-0.912 \mathrm{ML}
$$

(valid for $3.0<M L<5.9$ ). The shaded zone in figure 27 deppicts the 95 percent confidence interval for $\log (\mathrm{N})$.

The open circles in figure 27 give the cumulative number of earthquakes ( 59 earthquakes, 3. $0<M L<4.6)$ in the first 6 months of 1978 and the solid circles give the cumulative number of earthquakes $(96$ 


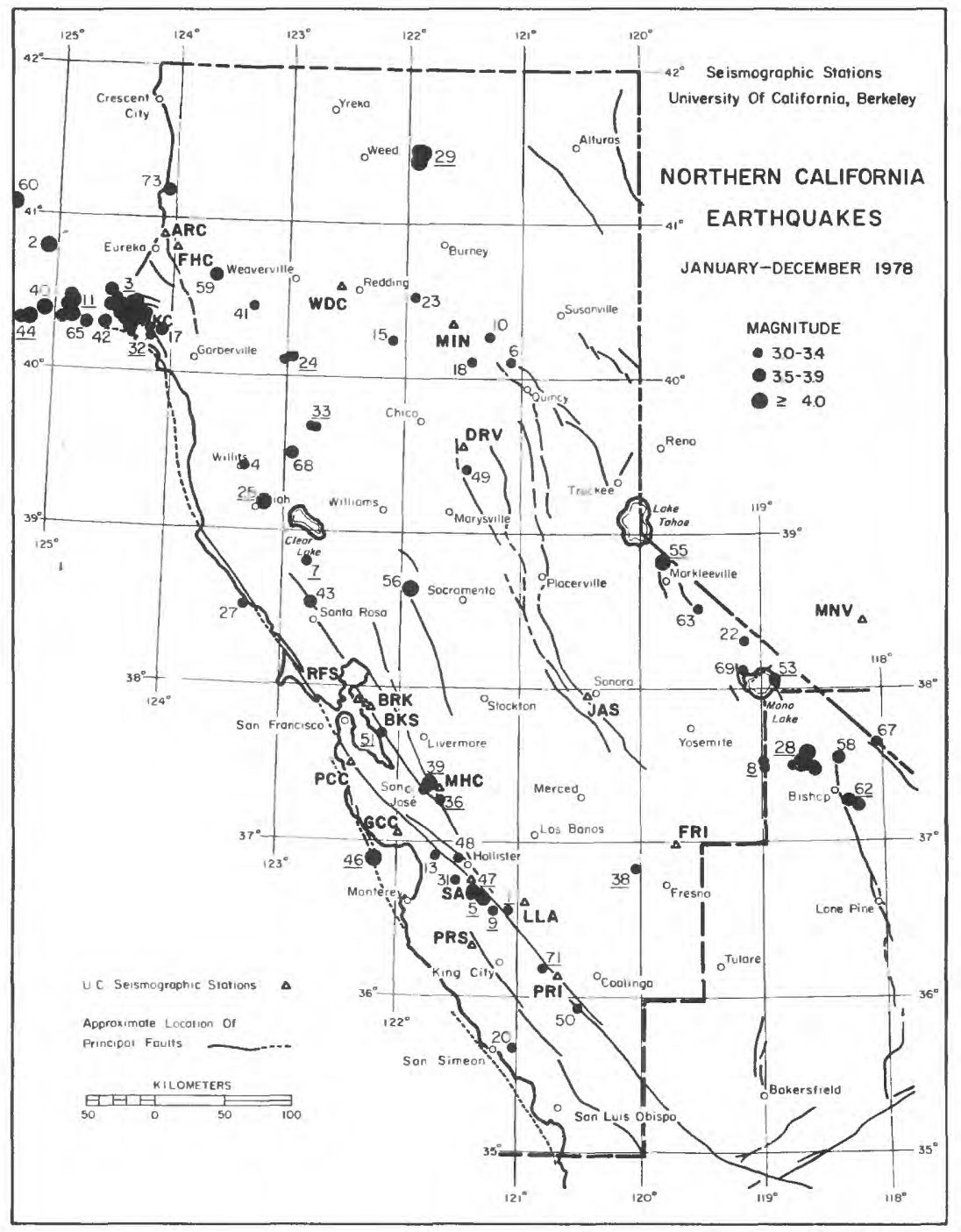

Figure 26.--Northern California earthquakes during 1978.

earthquakes $3.0<M L<5.3)$ in the last 6 months of 1978. There is thus no indication that the rate of seismicity for the first 6 months of 1978 is significantly different from the average semiannual rate of seismicity over the past decade. However, the rate of seismicity for the last 6 months of 1978 is significantly higher than the average semiannual rate of seismicity over the past decade. The high rate of seismicity for the last 6 months of 1978 (41 earthquakes, $3.0<M L<4.6)$ is attributed to the occurrence o $\bar{f}$ the earthquake sequence to the east of Mt. Shasta (\#29 in figure 26)

A sequence of 43 earthquakes (3.0 $<$ ML $\leq 5.8)$ centered approximately $30 \mathrm{~km}$ northwest of Bishop (\#28 in figure 26) occurred during the last quarter of 1978 . This sequence contained the largest earthquake (ML $=5.8$ ) to have occurred in northern and central California during 1978.
The Seismographic Station operated a network of 19 stations during 1978. Two instruments located at Berkeley (BKS) are of particular interest: a pair of WoodAnderson torsion instruments which are recorded on helicorders and on magnetic tape, and a three-component ultralongperiod seismograph ( $\mathrm{T}=100 \mathrm{sec})$ which is recorded photographically and on magnetic tape.

A pair of Wood-Anderson torsion seismometers are mounted in phototube amplifiers that provide electrical signals proportional to their deflection. The signals are recorded on helicorders at two magnification levels, $700 \mathrm{x}$ and $14000 \mathrm{x}$, and also on analog magnetic tape at two gain levels. The helicorder recordings are used for preliminary local magnitude (ML) estimates.

A three-component ultralong-period seismograph $(T=1,00 \mathrm{sec})$ is undergoing 
RECENT RATE OF SEISMICITY FOR NORTHERN \& CENTRAL CALIFORNIA

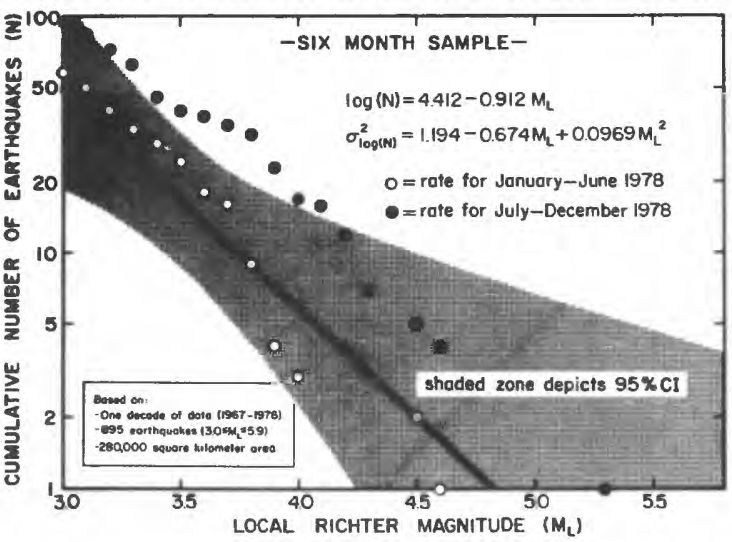

Figure 27.--Plot of cumulative number of earthquakes versus local Richter magnitude (ML).

continuous development. Displacement transducers are used on the seismometers to detect the boom deflection. The displacement transducer output is filtered to simulate a velocity transducer coupled to a 300 sec galvonometer and recorded photographically with a peak magnification of 500 at a period of $100 \mathrm{sec}$. The signals are also recorded on analog magnetic tape at two gain levels, +20 microns fullscale (flat in displacment response from $0.1 \mathrm{sec}$ to $100 \mathrm{sec)}$ and $+2 \mathrm{~mm}$ full-scale (passband: 0 - $10 \mathrm{~Hz}$ ). The ultralongperiod seismograph is used primarily to analyze major teleseisms and strong local earthquakes.

\section{SOUTHERN CALIFORNIA EARTHQUAKES, 1978}

By L. K. Hutton, J. H. Whitcomb, and $C$. R. Allen

Seismological Laboratory

California Institute of Technology

Pasadena, California 91125

and

G. S. Fuis and C. E. Johnson

U.S. Geological Survey at

Seismological Laboratory

California Institute of Technology

Pasadena, California 91125

During $1978,5,643$ local earthquakes were located by the 135-station Southern California Seismographic Network, which is operated jointly by the California Institute of Technology and the U.S. Geological Survey. Figure 28 shows these events plotted on a map of the region, with the major faults indicated. Caltech maintains an earthquake catalog complete above magnitude 3.0 in the area enclosed by the box in the figure.

Twenty-six of the year's earthquakes were 4.0 or greater in magnitude. The most significant sequences were: (1) a swarm in the Cerro Prieto area, Baja California, in March which included 288 events; (2) the Santa Barbara earthquake $(\mathrm{ML}=5.1)$ of August 13 and its 403 aftershocks; and (3) the Bishop (Owens valley) earthquake ( $M L=5.8)$ with its 223 aftershocks. The latter two main shocks caused light to moderate damage in their respective epicentral areas.

1978 marked the second year of routine operation of the CEDAR (Caltech Earthquake Detection and Recording) online computer system (Johnson, 1979). Experience shows that the seismicity data can be processed in a reasonably timely fashion, with a detection threshold maintained at 1.8 in the dense part of the network. The seismicity data are currently available in preliminary monthly catalogs (Whitcomb and others, 1978 , 1979), and will soon be printed in final form.

\section{CENTRAL MISSISSIPPI VALLEY EARTHQUAKES, 1978}

By W. Stauder, R. Herrmann, C. Nicholson,

S. Singh, M. Woods, C. Kim, R. Perry, S. Morrissey, and E. Haug Department of Earth and Atmospheric Sciences Saint Louis University St. Louis, MO 63156

During 1978, 244 earthquakes were located and 388 other nonlocatable earthquakes were detected by a twenty-four station regional microearthquake network operated by Saint Louis University under contract for the USGS and NRC. Figure 29 shows the earthquakes located within a $4^{\circ}$ $x \quad 4^{\circ}$ region centered on $37.0^{\circ} \mathrm{N}$. and $^{\circ}$ $89.5^{\circ} \mathrm{W}$. The magnitudes are indicated by the size of the open symbols. Figure 30 shows the locations and magnitudes of 210 earthquakes $10 \mathrm{cated}$ within a $1.5^{\circ} \mathrm{x} 1.5^{\circ}$ region centered at $36.25^{\circ} \mathrm{N}$. and $89.75^{\circ} \mathrm{W}$.

The year 1978 marked the beginning of expansion of the original 16 station USGS southeast Missouri regional seismic network by the addition of eight NRC sponsored stations in southeastern Illinois. Funding was also obtained from the USGS and the NRC to add 24 stations along the seismicity trends of figure 30. The resultant station separation of about 10 km will significantly improve hypocenter accuracies and detection thresholds.

Significant earthquakes during 1978 included the following:

1. 2 June 0207 UTC, $38.4^{\circ} \mathrm{N} ., 88.5^{\circ}$ W. f felt in Fairfield, Illinois, $\mathrm{m} 3 \mathrm{~Hz}=3.7$ (FVM).

2. 29 August 0705 UTC, $38.5^{\circ}$ N., $88.2^{\circ}$ W., felt in West Salem, Illinois, $\mathrm{m} 3 \mathrm{~Hz}=2.4$ (FVM). 


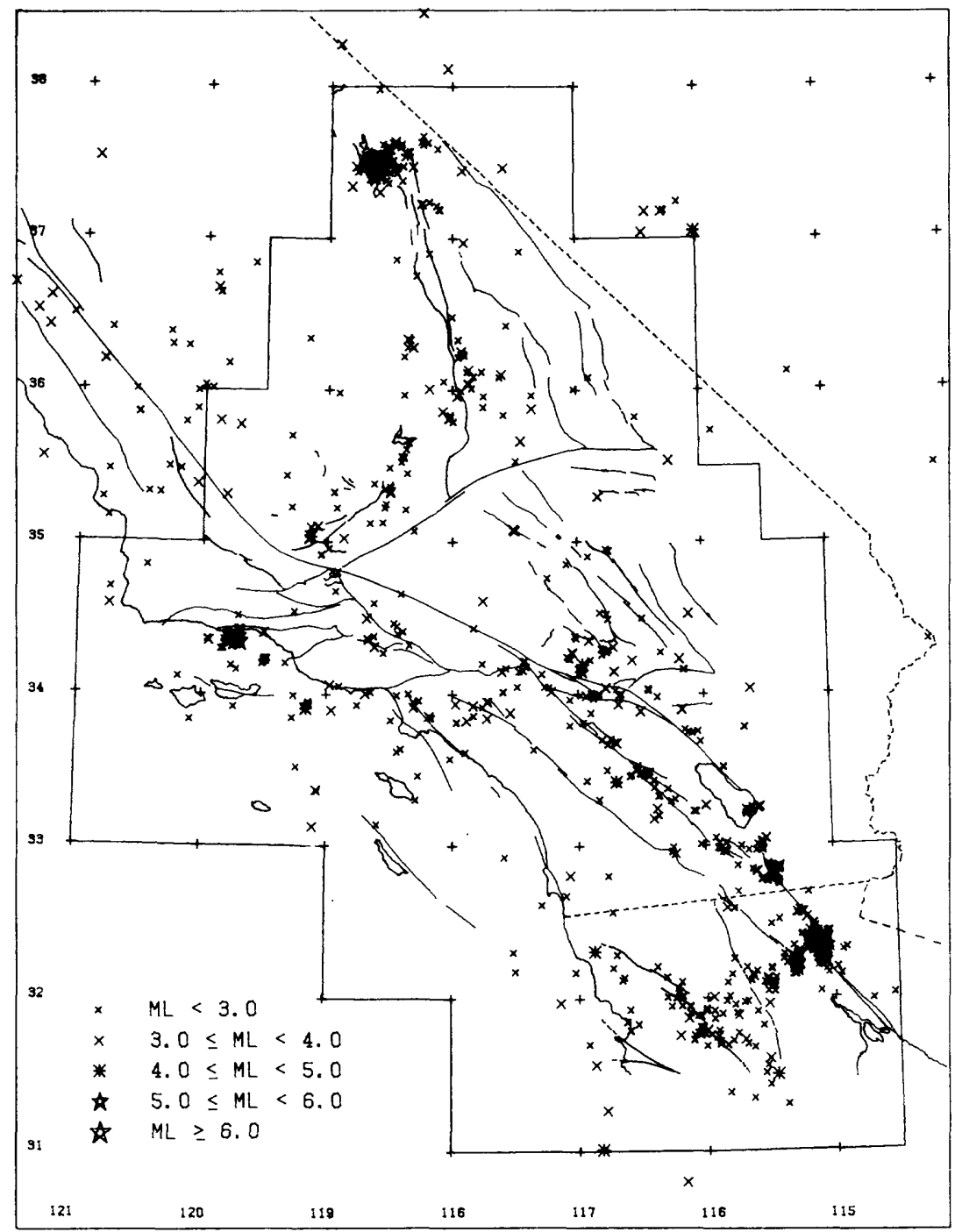

Figure 28.--Southern California earthquakes of magnitude 2.5 ML or greater during 1978 .

$89.4^{\circ}$ W., felt in Dyersburg, Tennessee, MM V, $\mathrm{mb}=3.5(\mathrm{FVM})$.

4. 20 September 1224 UTC, $38.6^{\circ} \mathrm{N}$, , $90.3^{\circ} \mathrm{W} .$, felt in St. Louis, Missouri, MM IV, $m b=3.0$ (BLA).

5. 21 November 2331 UTC, $36.0^{\circ} \mathrm{N}$, , 89.9 $9^{\circ} \mathrm{W}$., felt in Blytheville, Arkansas, $\mathrm{m} 3 \mathrm{~Hz}=2.3$ ( $\mathrm{FVM}$ ).

6. 5 December 0148 UTC, $38.6^{\circ}$ N., $88.4^{\circ}$ W., felt in West Salem, Illinois and neighboring five counties, $\mathrm{mb}=3.5$ (FVM).

The Illinois earthquakes located between WSIL and BPIL exhibit focal depths between 15 and $25 \mathrm{~km}$, making them deeper on the average than those in the Mississippi Embayment where the focal depths are usually less than $15 \mathrm{~km}$. A microearthquake survey using portable seismographs was conducted near Ridgely, Tennessee by Nicholson and Singh (1978). Northwest striking, southwesterly dipping reverse faulting was identified, near the station GRT of figure 30 , through the use of composite focal mechanisms and vertical depth profiles.

\section{KANSAS EARTHQUAKES, 1978}

By Don $W$. Steeples

Kansas Geological Survey University of Kansas

Lawrence, Kansas 66044

During 1978, the Kansas Geological Survey operated nine telemetered microearthquake seismograph stations ( $f i g$. $31)$ in eastern Kansas. Four additional stations were to be installed in northwest Kansas and south-central Nebraska during 1979. Station locations are given in table 2 . 


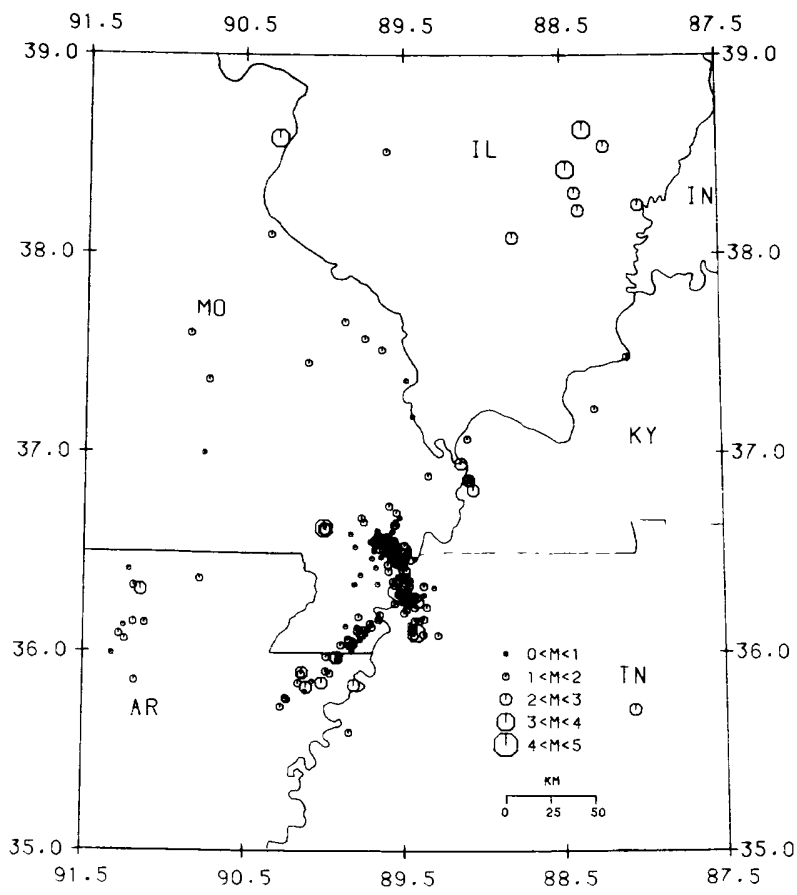

Fiqure 29.--Central Mississippi Valley earthquakes during 1978 within a $4^{\prime} \times 4^{\prime}$ region centered at $37.0^{\prime} \mathrm{N}$. and $89.5^{\prime}$ W.

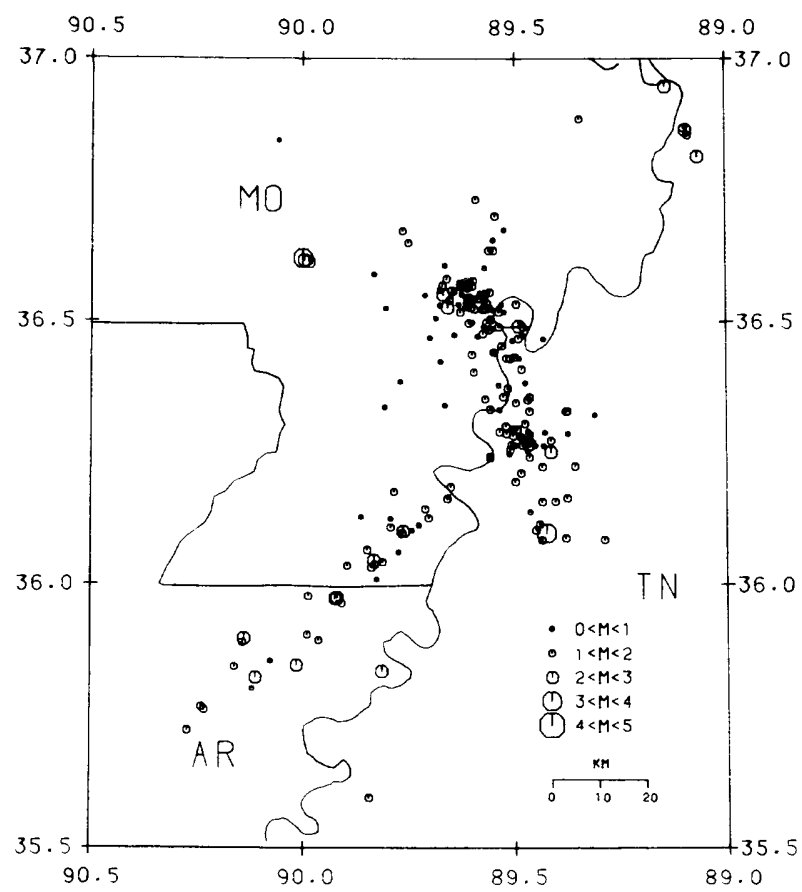

Figure 30.--Central Mississippi Valley earthquakes during 1978 within a $1.5^{\prime} \times 1.5^{\prime}$ region centered at $36.25^{\prime} \mathrm{N}$. and $89.75^{\prime} \mathrm{W}$.
A series of 16 microearthquakes occurred within or near the Kansas borders between December 1, 1977 and the end of 1978 (fig. 32). Location parameters are given in table 3. Several of the microearthquake locations seem to trend along the Nemaha/Humboldt structure, indicating that parts of it are at least slightly active.

The occurrence of microearthquakes along the approximate trace of the Humboldt fault implies the possible occurrence in the past and future of larger earthquakes. Previously unknown felt reports from the 1867 so-called "Manhattan earthquake" (Modified Mercalli Intensity VII) suggest that the epicenter may have been east of Manhattan near the known Humboldt fault trace (DuBois and Wilson, 1978) rather than "22 miles northwest of Manhattan" (Merriam, 1956). Several years of microearthquake data will be required in conjunction with historical earthquake data to estimate the average return period for the 1867-type earthquake.

The series of felt earthquakes in the Manhattan vicinity in 1929 remain an enigma in regard to their structural source. Isoseismal patterns clearly indicate that the epicenters were 20 to $40 \mathrm{~km}$ west of the trace of the Humboldt fault. It is expected that this question will be answered in the future by microearthquake recording.

The microearthquakes plotted in figure 32 in northwest Kansas and southwest Nebraska occurred between December 1, 1977 and September 30, 1978. The events are only located with an estimated accuracy of 20 to $25 \mathrm{~km}$, but they all clearly lie on a structural trend known as the Central Kansas Uplift in Kansas and as the Chadron and Cambridge Arch in Nebraska. On May 7 , 1978 an event with $\mathrm{mbLg}=3.9$ (not shown in fig. 32) occurred on the Chadron Arch in western Nebraska. The seismicity pattern of 1977-78, coupled with several historical epicenters (Woollard, 1958) suggests that some low to moderate level of tectonic activity is occurring along this whole structure in central and northwest Kansas and in western Nebraska. A longterm trend of such tectonic movement is explicitly suggested by stanley and Wayne (1972) on the basis of drainage and sedimentation patterns in the area during the Pleistocene Epoch and by present-day stream gradients and knickpoints on several rivers that cross the structural trend. 


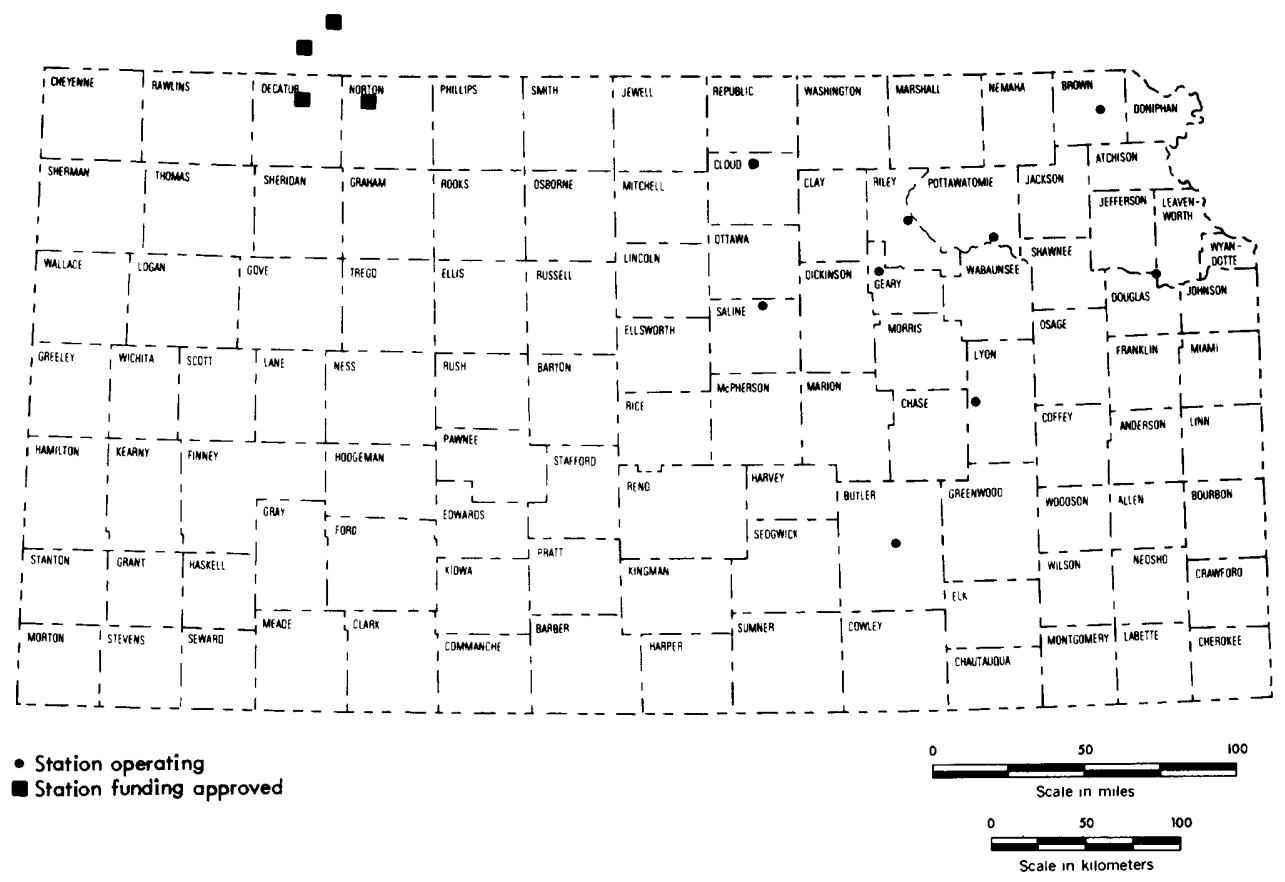

Figure 31.--Kansas Geological Survey seismograph network in 1978. Stations in Cloud, Saline, and Butler Counties did not begin operating until April, 1978 . Stations shown by squares were to be added in 1979 .
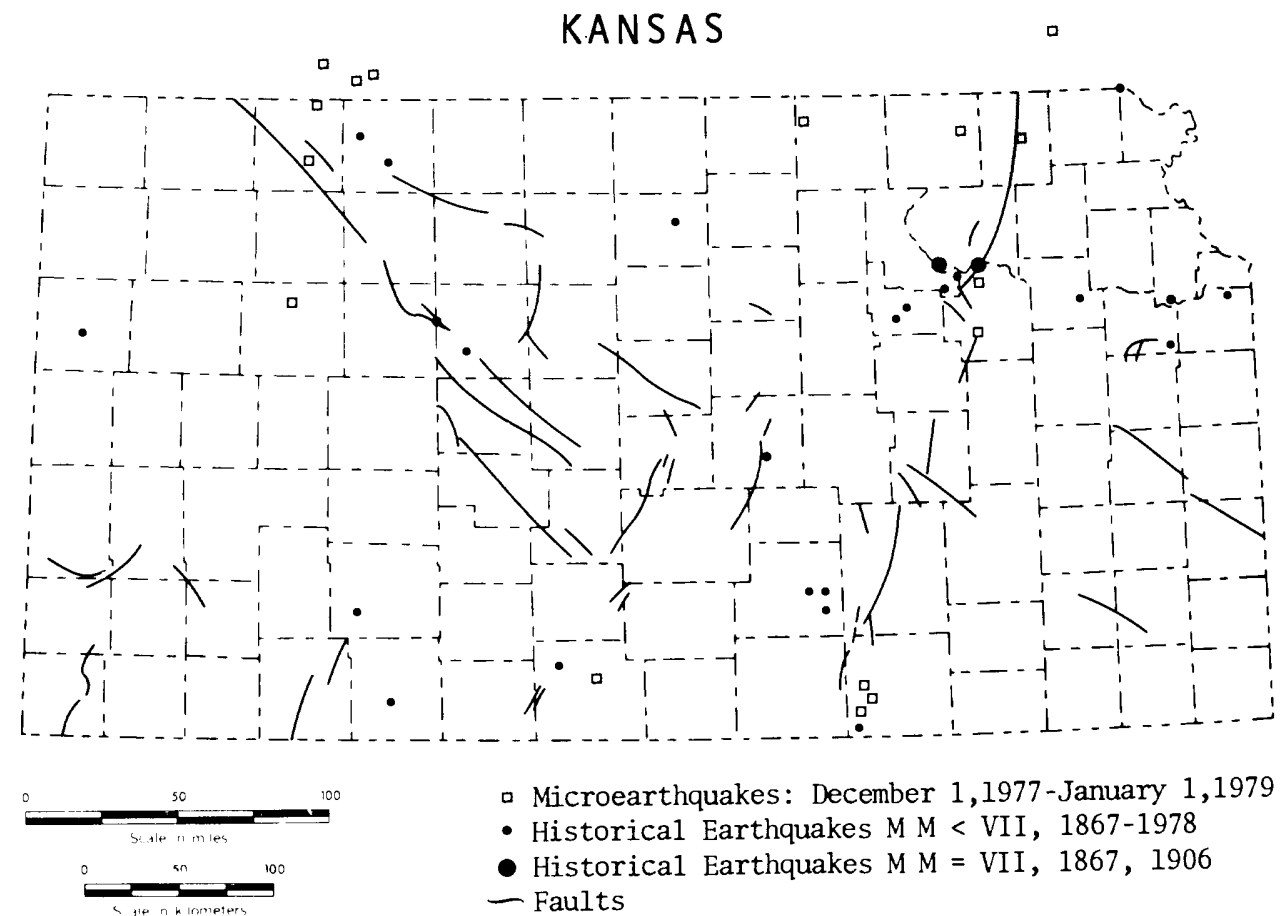

- Microearthquakes: December 1,1977-January 1,1979

- Historical Earthquakes M M < VII, 1867-1978

- Historical Earthquakes M M = VII, 1867, 1906

- Faults

Figure 32.--Kansas earthquakes and the Humboldt Fault Zone that trends northnortheasterly across the State. The Central Kansas Uplift is associated with the faults that trend north-westerly in northwestern Kansas. 
Table 2.--Station locations in Kansas

\begin{tabular}{cccc} 
Station & $\begin{array}{l}\text { Lat. } \\
\left({ }^{\circ} \mathrm{N}\right)\end{array}$ & $\begin{array}{l}\text { Long. } \\
\left({ }^{\circ} \mathrm{W}\right)\end{array}$ & $\begin{array}{l}\text { Elevation } \\
(\mathrm{m})\end{array}$ \\
BEK & 39.263 & 96.200 & 349 \\
EMK & 38.447 & 96.317 & 307 \\
HWK & 39.802 & 95.497 & 320 \\
LAK & 39.046 & 95.205 & 326 \\
MLK & 39.106 & 96.892 & 386 \\
TCK & 39.385 & 96.723 & 377 \\
EDK & 37.774 & 96.795 & 418 \\
SNK & 38.953 & 97.603 & 407 \\
CNK & 39.508 & 97.713 & 465 \\
OBK & 39.929 & 100.439 & 805 \\
NNK & 39.936 & 100.042 & 730 \\
\hline
\end{tabular}

Table 3.--Microearthquake locations in Kansas

\begin{tabular}{|c|c|c|c|c|c|c|c|}
\hline $\begin{array}{l}\text { Date } \\
(1978)\end{array}$ & \multicolumn{3}{|c|}{$\begin{array}{l}\text { Origin time } \\
\text { (UTC) }\end{array}$} & $\begin{array}{l}\text { Lat. } \\
\left({ }^{\circ} \mathrm{N} .\right)\end{array}$ & $\begin{array}{l}\text { Long } \\
\left({ }^{\circ} \mathrm{W} .\right)\end{array}$ & $\begin{array}{l}{ }^{2} \text { Depth } \\
(\mathrm{km})\end{array}$ & $\begin{array}{l}\text { Mag : } \\
\text { (DUR) }\end{array}$ \\
\hline Jan. & 21 & 32 & 03.88 & 38.868 & 96.201 & 0.41 & 1.6 \\
\hline Jan. & 20 & 15 & 33.39 & 40.093 & 95.700 & 5.00 & 1.7 \\
\hline Jan. & 11 & 25 & 37.65 & 39.836 & 95.974 & 9.03 & 2.4 \\
\hline Feb. & 00 & 25 & 47.62 & 40.032 & 100.333 & 5.00 & 2.4 \\
\hline Apr. 14 & 23 & 27 & 38.29 & 39.814 & 96.395 & 1.18 & 1.9 \\
\hline May 20 & 01 & 53 & 43.36 & 39.998 & 100.333 & 5.00 & 2.3 \\
\hline May & 04 & 28 & 35.06 & 39.135 & 96.292 & 8.86 & 2.3 \\
\hline Sep. & 08 & 06 & 18.59 & 40.896 & 100.367 & 5.00 & 2.2 \\
\hline Nov. & 08 & 44 & 59.88 & .860 & 97.349 & 5.00 & 1.6 \\
\hline Dec. & 23 & 06 & 14.64 & 37.340 & 98.640 & 0.52 & \\
\hline Dec. & 23 & 06 & 23.20 & 39.138 & 100.455 & 5.00 & 2. \\
\hline Dec. 10 & 13 & 41 & 02.12 & 39.477 & 93.259 & 5.00 & 2.0 \\
\hline
\end{tabular}

OKLAHOMA EARTHQUAKES, 1978

By James F. Lawson, Ir. Oklahoma Geophysical Observatory University of oklahoma Leonard, Okl ahoma

and

Kenneth V. Luza

Oklahoma Geological Survey

University of oklahoma

Norman, Okl ahoma 73019

The article below is a condensed version of a paper by Irawson and Luza originally published in oklahoma Geology Notes, vol. 39, June 1979 .

A statewide network of 10 seismograph stations are recording seismological data in Oklahoma (fig. 33). The Oklahoma Geophys-ical observatory station, TUL, has been recording earthquake data since December 1961. The Observatory, located near Leonard, Oklahoma, in southern Tulsa County, operates seven seismometers, three long-period and four short-period, which are installed in a vault detached from the main building. The seismic responses at TUL are recorded on 11 paper-drum recorders; 16 seismograms are recorded on 16-mm film. Seven semipermanent, volunteer- operated seismograph stations and two radio-telemetry stations constitute oklahoma's regional network. The installation and maintenance of these stations are being supported by the U.S. Nuclear Regulatory Commission (NRC) (Luza, 1978).

Each of the seven volunteer-operated seismograph stations consists of a Geotech S-13 short-period, vertical seismometer; a Sprengnether MEQ-800 B unit, including amplifier, filters, ink-recording unit and a clock; and a Kinemetrics time-signal radio receiver for high-frequency WWV time signals. Each radio-telemetry system consists of one Geotech $\mathrm{S}-13$ seismometer and one Emheiser Rand telemetry unit. Seismographs from the radio-telemetry stations are recorded at the okl ahoma Geophysical observatory.

In 1978,35 oklahoma earthquakes were located (fig. 34) by the Oklahoma Geophysical Observatory staff. Magnitude values range from a low of $1.3(\mathrm{~m} 3 \mathrm{~Hz})$. in okfuskee county to as high as 3.1 (m3Hz) in Le Flore county. The listing only represents those earthquakes that could be located by using three or more seismograph records. Only four earthquakes were reported felt by people living in the vicinity of the earthquake epicenters. These earthquakes include the March 9 earthquake in Love County, MM II (modified Mercalli intensity), the May 18 earthquake in Canadian County, MM I, and two earthquakes on May 19 in Canadian County, MM III and MM II.

The 1978 earthquake epicentral data, which form a limited data set, when combined with previous earthquake data produce some seismic trends worthy of discussion. Five 1978 earthquakes, of which three were felt, occurred near El Reno in Canadian county. This location is near the site of the April 9, 1952 earthquake with the magnitude of 5.5 (mb). Three additional earthquakes occurred northeast of El Reno in Logan County. These earthquakes, when combined with the historical earthquakes in this area, form a zone 30 $\mathrm{km}$ wide and $145 \mathrm{~km}$ long that crosses the Nemaha Uplift at a $30^{\circ}$ angle. It is not clear what this trend represents; however, it is subparallel to a a regional northeast-southwest structural grain in northeastern oklahoma. In south-central oklahoma, there is a concentration of earthquakes in the Wilson area, Carter and Love Counties. This area has also been the site of numerous small earthquakes in the past. The subsurface geology near wilson consists of deformed, complexly faulted Paleozoic rocks. Several 1978 epicenters as well as pre-1978 earthquake epicenters fall within and (or) along the northern front of the Ouachita Mountains. Several of these epicenters are within 1 or $2 \mathrm{~km}$ of known surface faulting. 


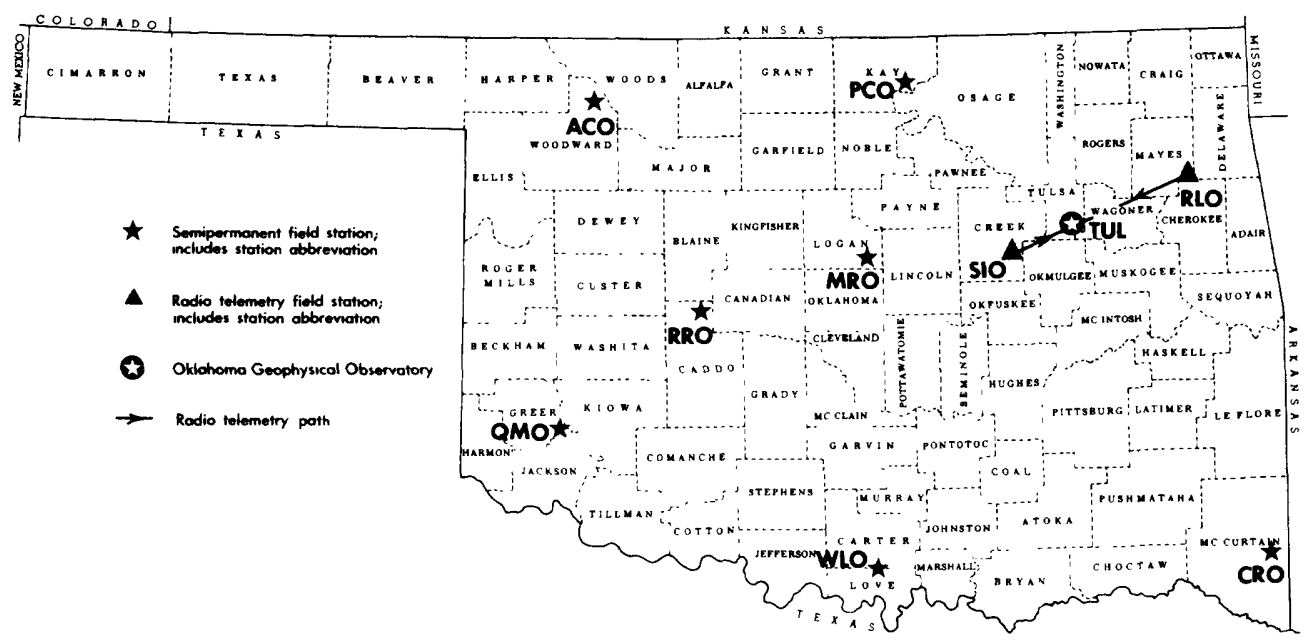

Figure 33.--Active seismograph stations in Oklahoma.

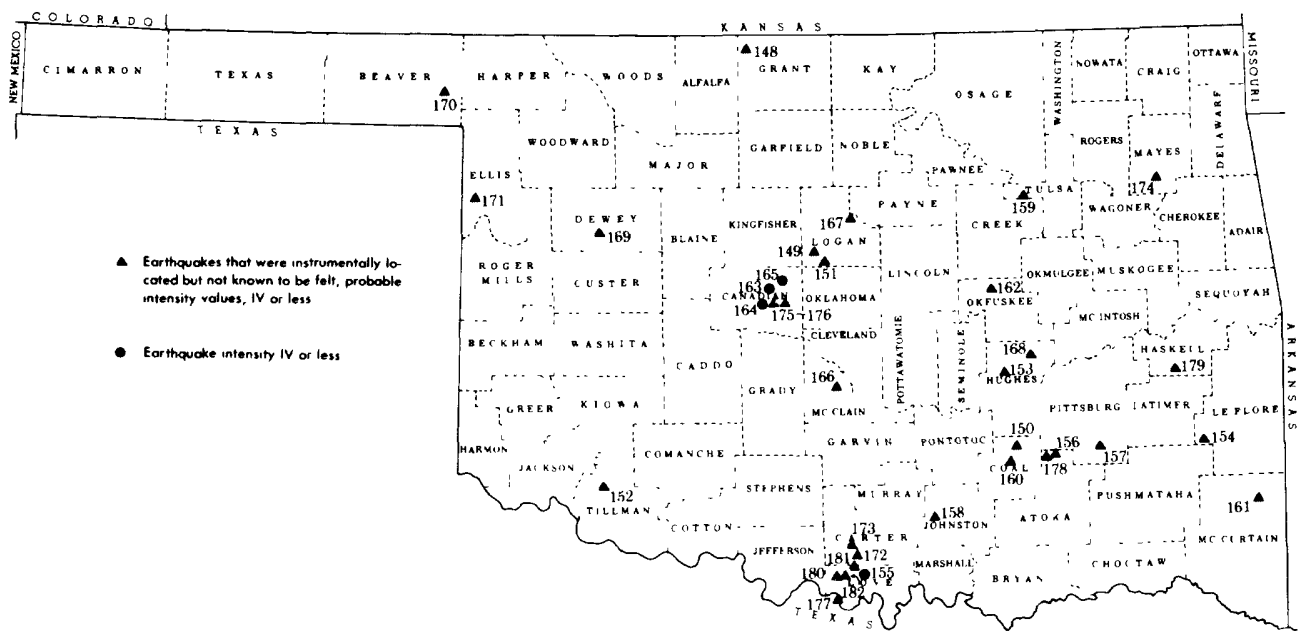

igure 34.--Distribution of Oklahoma earthquakes during 1978. Numbers correspond to event numbers in table 4 .

is used to calculate local earthquake epicenters. A catalog containing date, origin time, county, intensity, magnitude, location, focal depth, and references is printed in page-size format. Table 4 contains the 1978 Oklahoma earthquakes displayed in a modified version of the regional earthquake catalog.

There are several different scales used to report magnitude. Table 4 has three magnitude scales which are mbLg (Nuttli, 1973), m3Hz (Nuttli, 1973), and MDUR (Lawson, 1978). Each magnitude scale was established to accommodate specific criteria, such as the distance from the epicenter, as well as the availability of certain seismic data.

For earthquake epicenters located from $11 \mathrm{~km}$ to $222 \mathrm{~km}$ from a seismograph station, otto Nuttli developed the $\mathrm{m} 3 \mathrm{~Hz}$ magnitude scale (Zollweg, 1974). This magnitude is derived from the following expression:

$$
m 3 \mathrm{~Hz}=\log (\mathrm{A} / \mathrm{T})-1.63+.87 \log (\mathrm{D}),
$$

where $A$ is the maximum center-to-peak vertical-ground-motion amplitude sustained for three or more cycles of $\mathrm{Sg}$ waves, near 3 hertz in frequency, measured in nanometers; $T$ is the period of the $S g$ waves measured in seconds; and $D$ is the greatcircle distance from epicenter to station measured in kilometers.

Otto Nutt1i's (1973) earthquake magnitude, mbla, for seismograph stations located between $55.6 \mathrm{~km}$ and $445 \mathrm{~km}$ from the epicenter, is derived from the following equation:

$$
m b L g=\log (A / T)-1.09+0.90 \log (D) .
$$

Where seismograph stations are located 
between $445 \mathrm{~km}$ and $3,360 \mathrm{~km}$ from the epicenter, mbLg is defined as

$$
\mathrm{mbIg}=\log (\mathrm{A} / \mathrm{T})-3.10+1.66 \log (D),
$$

where $A$ is the maximum center-to-peak vertical-ground-motion amplitude sustained for three or more cycles of Sg waves, near 1 hertz in frequency, measured in nanometers; $\mathrm{T}$ is the period of $\mathrm{Sg}$ waves measured in seconds; and is the great-circle distance from station to epicenter measured in kilometers.

The MDUR magnitude scale was developed by Lawson (1978) for earthquakes in Oklahoma and adjacent areas. It is defined as

$$
\text { MDUR }=1.86 \log (\text { DUR })-1.49,
$$

where DUR is the duration or difference, in seconds, between the Pg-wave arrival time and the time the final coda amplitude decreases to twice the background-noise amplitude. If the $P n$ wave is the first arrival, the interval between the earthquake-origin time and the decrease of the coda to twice the background-noise amplitude is measured instead.

The depth to the earthquake hypocenter is measured in kilometers. For most Oklahoma earthquakes the focal depth is unknown. In almost all oklahoma events, the stations are several times farther from the epicenter than the likely depth of the event. This makes the locations indeterminate at depth, which usually requires that the hypocenter depth be restrained to an arbitrary $5 \mathrm{~km}$ for purposes of computing latitude, longitude, and origin time. All available evidence indicates that no Oklahoma hypocenters have been deeper than 15 to $20 \mathrm{~km}$.

\begin{tabular}{|c|c|c|c|c|c|c|c|c|c|c|c|c|c|c|}
\hline $\begin{array}{l}\text { Event } \\
\text { number }\end{array}$ & \multicolumn{2}{|c|}{$\begin{array}{l}\text { Date } \\
(1978)\end{array}$} & \multicolumn{3}{|c|}{$\begin{array}{l}\text { Origin time } \\
\text { (UTC) }\end{array}$} & $\begin{array}{l}\text { Lat } \\
(\mathrm{N} .)\end{array}$ & $\begin{array}{l}\text { Long } \\
(\mathrm{W} .)\end{array}$ & \multicolumn{2}{|c|}{$\begin{array}{l}\text { Depth } \\
(\mathrm{km})\end{array}$} & \multicolumn{3}{|c|}{ Magnitudes } & County & $\begin{array}{l}\text { Intensity } \\
\text { (MM) }\end{array}$ \\
\hline 148 & Jan. & 8 & 04 & 16 & 33.56 & 36.971 & 97.463 & 5.0 & $\mathrm{R}$ & 1.5 & -- & 1.5 & Kay & --- \\
\hline 149 & Jan. & 8 & 10 & 19 & 17.65 & 35.824 & 97.642 & 5.0 & $\mathrm{R}$ & 2.1 & 2.0 & 2.2 & Log an & -- \\
\hline 150 & Feb. & 10 & 06 & 42 & 02.39 & 34.712 & 96.157 & 5.0 & $\mathrm{R}$ & 2.1 & 1.5 & 1.9 & Coal & --- \\
\hline 151 & Feb. & 14 & 01 & 09 & 38.64 & 35.777 & 97.585 & 5.0 & $\mathrm{R}$ & 1.7 & $-\infty$ & 1.7 & Logan & --- \\
\hline 152 & Feb. & 21 & 11 & 12 & 48.11 & 34.535 & 99.003 & 5.0 & $\mathrm{R}$ & 2.5 & 2.2 & 2.0 & Tillman & --- \\
\hline 153 & Mar. & 3 & 02 & 24 & 37.28 & 35.086 & 96.278 & 5.0 & $\mathrm{R}$ & 2.5 & 2.1 & 2.4 & Hughes & $-\infty$ \\
\hline 154 & Mar. & 5 & 14 & 46 & 50.48 & 34.699 & 95.033 & 7.0 & $\mathrm{R}$ & 3.1 & 2.9 & 2.7 & LeFlore & --- \\
\hline 155 & Mar. & 9 & 06 & 30 & 50.82 & 34.010 & 97.378 & 5.0 & $R$ & -- & 2.6 & 2.5 & Love & II \\
\hline 156 & Apr. & 2 & 21 & 32 & 48.08 & 34.635 & 96.057 & 5.0 & $\mathrm{R}$ & 2.5 & 2.3 & 2.5 & Atoka & $-\infty$ \\
\hline 157 & Apr. & 11 & 08 & 51 & 02.43 & 34.693 & 95.681 & 5.0 & $\mathrm{R}$ & 1.7 & -- & 1.8 & Pittsburg & --- \\
\hline 158 & Apr. & 13 & 03 & 43 & 50.76 & 34.351 & 96.820 & 5.0 & $\mathrm{R}$ & 1.9 & 2.0 & 1.9 & Johnston & --- \\
\hline 159 & Apr. & 19 & 14 & 20 & 54.06 & 36.088 & 96.136 & 5.0 & $\mathrm{R}$ & 1.5 & -- & 1.1 & Tulsa & -- \\
\hline 160 & Apr. & 20 & 08 & 13 & 04.00 & 34.586 & 95.293 & 5.0 & $R$ & 1.7 & -- & 1.6 & Coal & --- \\
\hline 161 & May & 1 & 22 & 59 & 13.38 & 34.400 & 94.673 & 5.0 & $\mathrm{R}$ & 2.1 & 2.2 & 2.2 & McCurtain & --- \\
\hline 162 & May & 4 & 04 & 35 & 52.89 & 35.588 & 96.345 & 5.0 & $\mathrm{R}$ & 1.3 & -- & 1.5 & Okfuskee & --- \\
\hline 163 & May & 17 & 23 & 11 & 15.65 & 35.525 & 97.910 & 5.0 & $\mathrm{R}$ & 2.1 & 2.3 & 2.0 & Canadian & I \\
\hline 164 & May & 18 & 00 & 19 & 22.43 & 35.502 & 97.949 & 5.0 & $\mathrm{R}$ & 2.5 & 2.7 & 2.6 & Canadian & I I I \\
\hline 165 & May & 18 & 00 & 32 & 17.57 & 35.601 & 97.828 & 5.0 & $\mathrm{R}$ & 2.2 & 2.1 & 2.1 & Canadian & II \\
\hline 166 & May & 19 & 00 & 39 & 37.46 & 35.135 & 97.503 & 5.0 & $\mathrm{R}$ & 1.7 & 2.0 & 1.9 & McCla in & --- \\
\hline 167 & May & 19 & 06 & 27 & 32.70 & 36.002 & 97.367 & 5.0 & $\mathrm{R}$ & 1.8 & --- & 1.4 & Log an & --- \\
\hline 168 & May & 2.8 & 09 & 19 & 00.22 & 35.213 & 96.144 & 5.0 & $\mathrm{R}$ & 2.1 & -- & 1.8 & Hughes & --- \\
\hline 169 & June & 22 & 05 & 10 & 15.54 & 35.923 & 99.089 & 5.0 & $\mathrm{R}$ & 2.0 & $-\infty$ & 2.2 & Dewey & $-\infty$ \\
\hline 170 & Aug. & 3 & 00 & 35 & 37.09 & 36.689 & 100.162 & 5.0 & $\mathrm{R}$ & 2.3 & 2.1 & 2.4 & Beaver & --- \\
\hline 171 & Aug. & 6 & 04 & 28 & 56.83 & 36.073 & 99.935 & 5.0 & $\mathrm{R}$ & 3.0 & 2.2 & 2.6 & Ellis & -- \\
\hline 172 & Aug. & 8 & 12 & 07 & 48.69 & 34.127 & 97.463 & 5.0 & $\mathrm{R}$ & 2.3 & 2.2 & 1.9 & Carter & --- \\
\hline 173 & Aug. & 26 & 14 & 57 & 51.99 & 34.178 & 97.463 & 5.0 & $\mathrm{R}$ & -- & --- & 1.4 & Carter & --- \\
\hline 174 & Sep. & 8 & 05 & 16 & 06.60 & 36.155 & 95.275 & 5.0 & $\mathrm{R}$ & -- & -- & 1.4 & Mayes & --- \\
\hline 175 & Sep. & 26 & 21 & 17 & 17.72 & 35.519 & 97.866 & 5.0 & $\mathrm{R}$ & 2.2 & 2.2 & 2.2 & Canadian & --- \\
\hline 176 & Sep. & 27 & 01 & 56 & 03.81 & 35.519 & 97.843 & 5.0 & $\mathrm{R}$ & 2.2 & 2.1 & 2.2 & Canadian & -- \\
\hline 177 & Sep. & 27 & 20 & 56 & 03.75 & 33.883 & 97.477 & 5.0 & $\mathrm{R}$ & 2.4 & --- & 1.9 & Love & -- \\
\hline 178 & Dec. & 8 & 11 & 18 & 53.92 & 34.676 & 96.063 & 5.0 & $\mathrm{R}$ & 2.0 & 1.8 & 1.7 & Atoka & $-\infty$ \\
\hline 179 & Dec. & 19 & 02 & 00 & 28.87 & 35.086 & 95.125 & 5.0 & $\mathrm{R}$ & 1.2 & 1.7 & 1.7 & Haskell & -- \\
\hline 180 & Dec. & 27 & 22 & 00 & 30.02 & 33.996 & 97.512 & 5.0 & $\mathrm{R}$ & 2.0 & $-\infty$ & 1.9 & Love & --- \\
\hline 181 & Dec. & 28 & 05 & 30 & 32.43 & 34.080 & 97.462 & 5.0 & $\mathrm{R}$ & 1.4 & 1.9 & 1.5 & Love & --- \\
\hline 182 & Dec. & 2.8 & 13 & 54 & 09.81 & 33.991 & 97.456 & 5.0 & $\mathrm{R}$ & 1.9 & 2.1 & 1.9 & Love & -- \\
\hline
\end{tabular}

Table 4.--Oklahoma earthquake catalog for 1978 


\section{SOUTHEASTERN UNITED STATES EARTHQUAKES. 1978}

By G. A. Bollinger Department of Geological Sciences Virg inia Polytechnic Institute and State University Blacksburg, virginia 24061

There were 25 earthquakes located in the southeastern United States during 1978. Of these, seven had $M \geq 3$ and/or were felt (table 5). Considerable microearthquake $(M<3)$ activity was reported near reservoírs in South Carolina and Georgia. Finally, some microearthquake occurrences, not in the vicinity of reservoirs, were reported in virginia. Figure 35 shows the spatial distribution of the 1978 seismicity. A full data listing for all 1978 shocks was given in Southeastern United States Seismic Network Bulletins No. 2 and 3. Copies of those reports may be obtained by contacting the author.

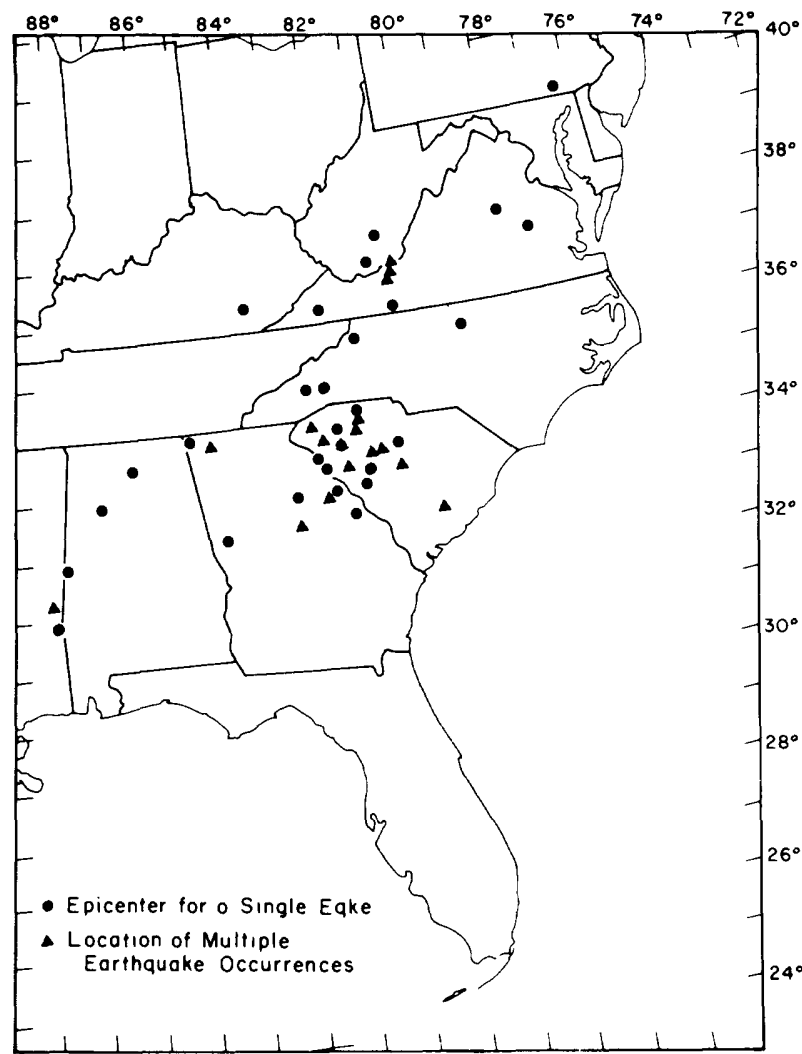

Figure 35.--Southeastern United States earthquake epicenters during 1978 .
The number of seismic stations operating in the region varied between 62 and 81 . Figure 36 shows the station distribution (81) present at the end of 1978.

Table 5.--Southeastern United States earthquakes

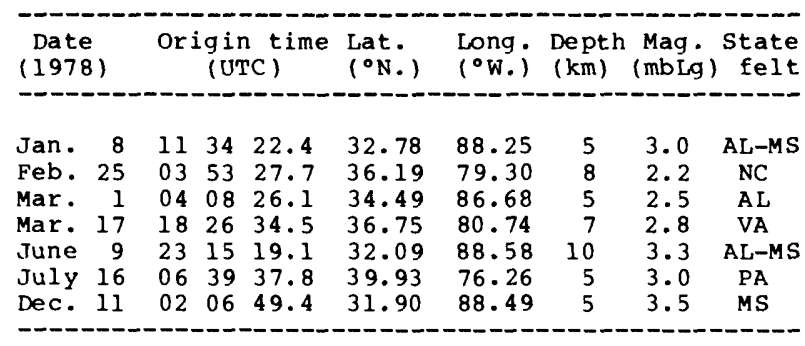

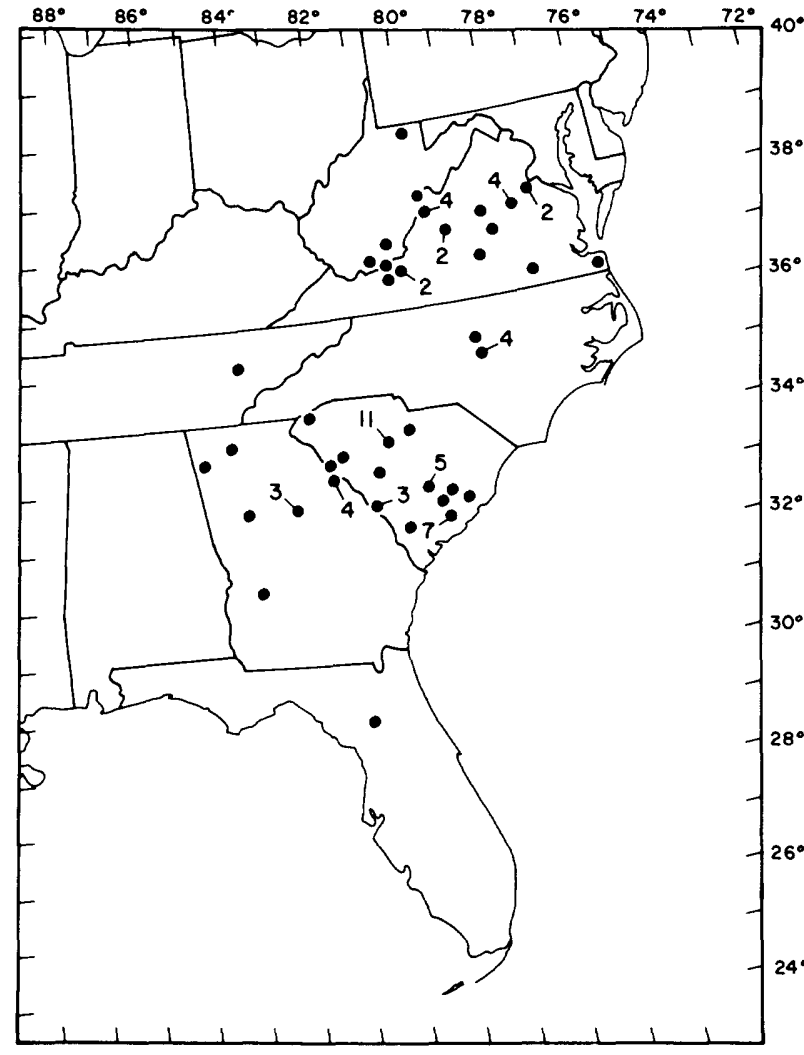

Figure 36.--Southeastern United States seismic stations (solid circles) operating at the close of 1978. Numbers indicate the number of closely-spaced multiple stations at a given location. 


\section{VIRGINIA EARTHQUAKES, 1978}

By G. A. Bollinger

Department of Geological Sciences Virginia Polytechnic Institute and State University

Blacksburg, virginia 24061

Installation of a 10-station seismic network in Virginia was completed in 1978. That network consists of two 5-station clusters: One in the Giles county area of western Virginia and the other in the central portion of the state. Additionally, 4-station subarrays, in the North Anna (central Virginia) and Bath County (north central Virginia), were obtained from Virginia Electric and Power Company. By the end of 1978,16 of the 18 total stations were operational (see fig. 37).

During this initial monitoring period, a total of 26 earthquakes ($0.9<\mathrm{M}<2.8)$ were detected. Of these, only 11 were locatable (three or more recording stations; see fig. 38). Their HYPO 71 parameters are shown in table 6 . For the remaining 15 nonlocatable events, 8 were in the Giles County area, 3 were in central Virginia, 1 was near North Anna, and 3 were in Bath County.

Table 6.--Virginia earthquakes

$\begin{array}{lrrrrrrr}\begin{array}{c}\text { Date } \\ \text { (1978) }\end{array} & \begin{array}{c}\text { Origin time } \\ \text { (UTC) }\end{array} & \begin{array}{l}\text { Lat. } \\ \left({ }^{\circ} \mathrm{N} .\right)\end{array} & \begin{array}{l}\text { Long; } \\ \left({ }^{\circ} \mathrm{W} .\right)\end{array} & \begin{array}{r}\text { Depth Mag. } \\ \text { (km) }\end{array} \\ \text { (DUR) }\end{array}$

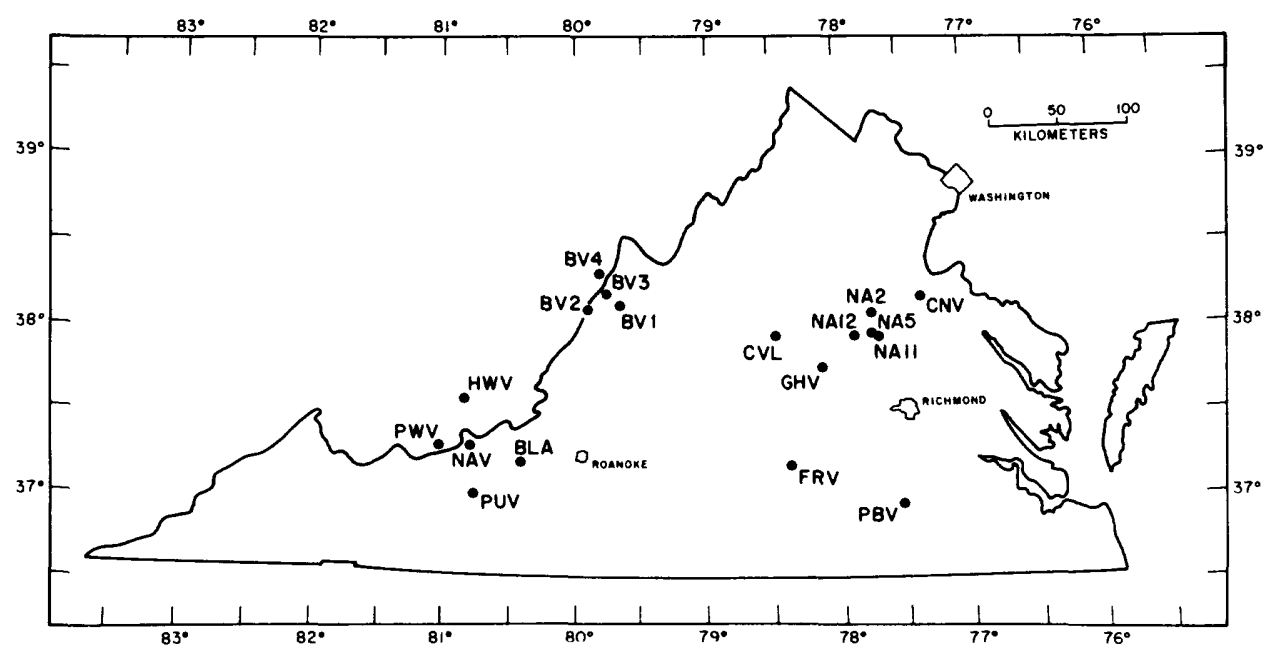

Figure 37.--Virginia Technical Seismic Network.

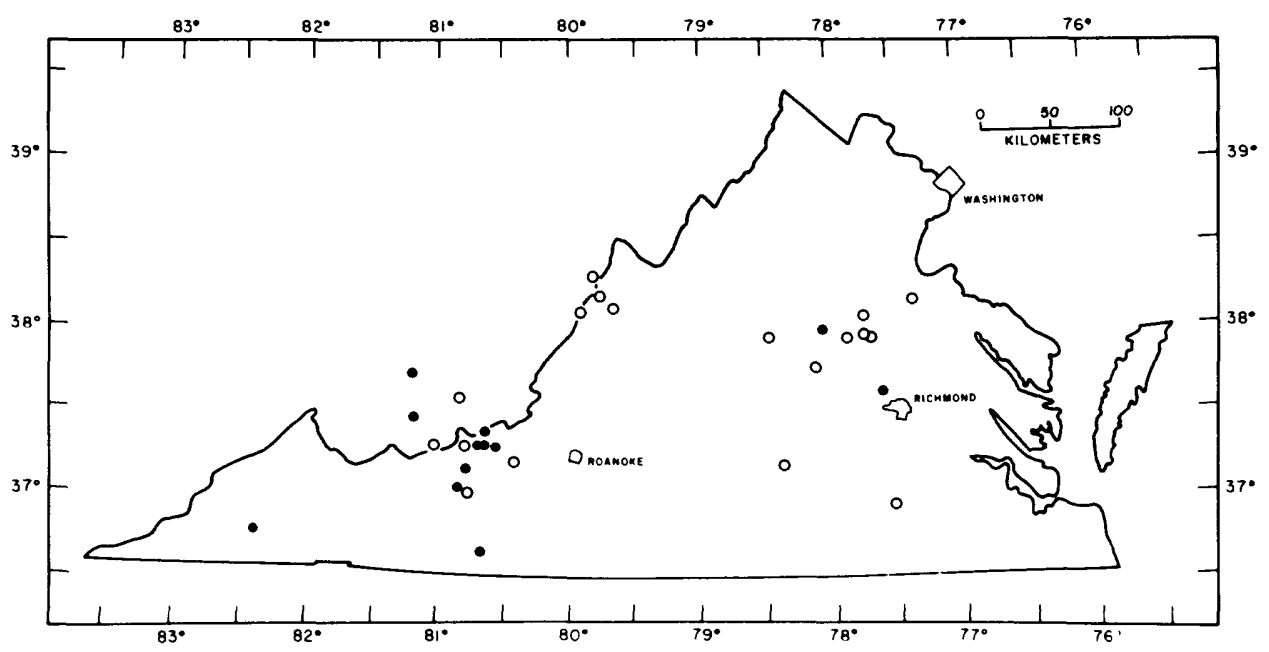

Figure 38.--Virginia earthquake epicenters during 1978. Solid circles represent earthquake epicenters and o en circles represent seismic stations. 


\section{Miscellaneous Activities}

\section{CRUSTAL MOVEMENT STUDIES}

\author{
Vertical Control Surveys
}

By Sandford R. Holdahl

NOAA, National Ocean Survey

National Geodetic Survey

Rockville, Maryland 20852

\section{Alaska}

The results of four leveling surveys carried out by the National Geodetic Survey between Anchorage and Whittier, Alaska, combined with an analysis of sea level measurements at Anchorage, indicate as much as $0.55 \mathrm{~m}$ of 1 and uplift in the decade following the 1964 prince william Sound earthquake. There is some indication that the position of maximum uplift is migrating away from Anchorage toward the Aleutian trench. The uplift occurs in a region which subsided as much as $1.9 \mathrm{~m}$ during the earthquake. The uplift is decreasing exponentially since the time of the 1964 earthquake, and seems to represent postseismic deformation. The observations are most easily explained by creep along the down dip extension of the fault which ruptured in 1964 .

Imperial Valley, California

A releveling survey of the Imperial valley was cooperatively accomplished in the winter of $1977-78$ by local and federal agencies. The purpose of the survey was to detect the trend of natural vertical motion prior to the utilization of geothermal resources in the valley. The economically significant agricultural development within the Imperial valley relies heavily on irrigation, and hence, it is directly affected by subsidence or tilting of the land. The continuation of the leveling program will measure the impact of geothermal development on the agricultural community. Existing data reveal that a maximum subsidence of $19 \mathrm{~cm}$ relative to Ocotillo occurred near the south end of the Salton sea for the 197277 time interval. A large area between the Salton Sea and El Centro has subsided more than $10 \mathrm{~cm}$ during the 5-year interval.

\section{Southern California}

In early 1978 , a massive $4,400 \mathrm{~km}$ releveling survey of the palmdale region of Southern California was bequn. The objective of the project was to establish a time-homodeneous reference network for calculating future elevation changes, and also to calculate recent vertical movement in the region which spans the San Andreas and Garlock faults. The 3-month, \$1.5 million, multiagency survey was coordinated by the National Geodetic survey, involved 18 leveling and 5 gravity teams from the National Geodetic Survey and the U.S. Geological Survey. In addition, 20 leveling teams were provided by Imperial, Los Angeles, Orange, Riverside, San Bernardino, San Diego, and Ventura counties, Los Angeles City, the Los Angeles Water and Power Department, and the Metropolitan Water District of Southern California in this cooperative effort. A joint report by the U.S. Geological survey and the National Geodetic Survey is in preparation.

Houston, Texas

Leveling surveys in approximately 5year intervals are being used to monitor subsidence in the Houston, Texas area. The maximum subsidence for the 1 ast inter$\mathrm{val}, 1973-78$, occurred approximately $25 \mathrm{~km}$ from the center of Houston and amounted to $43 \mathrm{~cm}$. An area of approximately $2500 \mathrm{sq}$ $\mathrm{km}$ has subsided more than $20 \mathrm{~cm}$ during this interval. In calculating the subsidence, the known rate of subsidence at the Galveston tide gauge was used to initialize the new set of heights; in addition, various points well outside Houston were assumed to be stable during the period.

Horizontal Control Surveys

By Richard A. Snay

NOAA, National Ocean Survey

National Geodetic Survey

Rockville, Maryland 20852

The National Geodetic Survey has recently observed fault monitoring networks in the Shelter Cove area of northern California and on the Tejon Ranch in the foothills of California's Tehachapi Mountains. The Shelter Cove network straddles part of the fault which ruptured during the 1906 San Francisco earthquake. Analysis of the recent survey data, combined with data from a 1930 network yields an estimate of $\mathrm{N} .13 .2^{\circ} \mathrm{W}$. $\pm 4.5^{\circ}$ for the direction of maximum $\bar{r}$ ight-lateral shear strain through the area. The mean shear strain rate in this direction is $1.01+0.18 \mu \mathrm{rad} / \mathrm{yr}$ for the $1930-76$ time inter $\bar{v} a l$.

The Tejon Ranch network spans two 
branches of the Garlock fault where this fault intersects the California aqueduct. The network incorporates two interior networks which were established in 1964 and 1970 , and have been repeatedly observed at one to three intervals. Network stations positioned on one side of the Garlock fault's southern branch have moved leftlaterally relative to stations lying approximately $500 \mathrm{~m}$ away on the opposite side, according to the following rates:

\begin{tabular}{cc} 
Time Interval & Rate (mm/yr) \\
\cline { 2 - 2 } $1964-68$ & $1.7 \pm 0.5$ \\
$1968-72$ & $1.7 \pm 0.6$ \\
$1972-78$ & $0.2 \pm 0.3$
\end{tabular}

The report for each survey is available from the National Geodetic Survey, National Ocean Survey, NOAA, Rockville, Maryland 20852 .

\section{Gravity Surveys}

By William E. Strange

National Ocean Survey

National Geodetic Survey

Rockville, Maryland 20852

In conjunction with large scale releveling surveys in southern California, the National Geodetic Survey established a network of high-accuracy gravity stations for future monitoring. The network included 29 stations which were continuously interconnected during the period of the survey using helicopter transportation. Measurements were made at 2180 benchmarks along the level lines using multiple gravimeters to interconnect to the helicopter stations. Information on this network is available from the National Geodetic Survey of the National Ocean Survey, Rockville, Maryland 20852.

\section{TSUNAMIS}

By Mark Spaeth

National Oceanic and Atmospheric Administration

National Weather Service Silver Spring, Maryland 20910

During 1978, seven tsunamis were reported to the National Oceanic and
Atmospheric Administration; however, none were recorded on NOAA tide gauges.

An earthquake on January 14 (mag. 6.6 MS) near the south coast of Honshu $\left(34.8^{\circ}\right.$ N., $139.3^{\circ}$ E.) caused a tsunami which was recorded at four nearby stations. Maximum amplitudes were $70 \mathrm{~cm}$ at Oshima and $25 \mathrm{~cm}$ at Mera.

On March 23, an earthquake (mag. 7.5 MS) in the Kuril Islands $\left(44.9^{\circ} \mathrm{N}, 148.4^{\circ}\right.$ E.) generated a tsunami which reached maximum amplitudes of $28 \mathrm{~cm}$ at Chichijima and Hanasaki and $25 \mathrm{~cm}$ at Yuzhno Kurilsk.

A second earthquake (mag. $7.6 \mathrm{MS}$ ) in the same area on March $24\left(44.2^{\circ} \mathrm{N}\right.$. $\left.148.9^{\circ} \mathrm{E}.\right)$ also caused a minor tsunami with maximum wave heights of $42 \mathrm{~cm}$ at Hanasaki and $30 \mathrm{~cm}$ at Chichijima.

The June 12 earthquake (mag. 7.7 MS) near the east coast of Honshu also caused a minor tsunami which was recorded with the following representative maximum amplitudes; Hachinohe, $42 \mathrm{~cm}$; Ayukawa, 34 $\mathrm{cm}$; Onahama and ofunato, $30 \mathrm{~cm}$; and Kushiro, $25 \mathrm{~cm}$.

The Mindanao earthquake $\left(8.2^{\circ} \mathrm{N}\right.$. $122.4^{\circ} \mathrm{E}$., mag. $\left.6.9 \mathrm{MS}\right)$ of June 14 caused a slight tsunami which was recorded at Jolo with a maximum amplitude of $3 \mathrm{~cm}$.

An earthquake (mag. $7.4 \mathrm{MS}$ ) on July 23 in the Taiwan region $\left(22.3^{\circ} \mathrm{N} ., 121.5^{\circ}\right.$ E.) caused a tsunami which reached a maximum amplitude of $8 \mathrm{~cm}$ at Ishigaki Shima.

The final tsunami of the year on November 29 was generated by an earthquake (mag. 7.7 MS) near Oaxaca, Mexico, (16. $0^{\circ}$ N., $96.6^{\circ}$ W.) and was recorded at Acajutla, El Salvador, with a maximum amplitude of $29 \mathrm{~cm}$.

\section{PRINCIPAL EARTHQUAKES OF THE WORLD}

Table 7 1ists principal world earthquakes for 1978. The 1 ist has been included in this annual series since 1941 . It includes earthquakes of magnitude 6.8 or greater; those of smaller magnitude that were locally destructive to 1 ife and property; and events of unusual interest. 


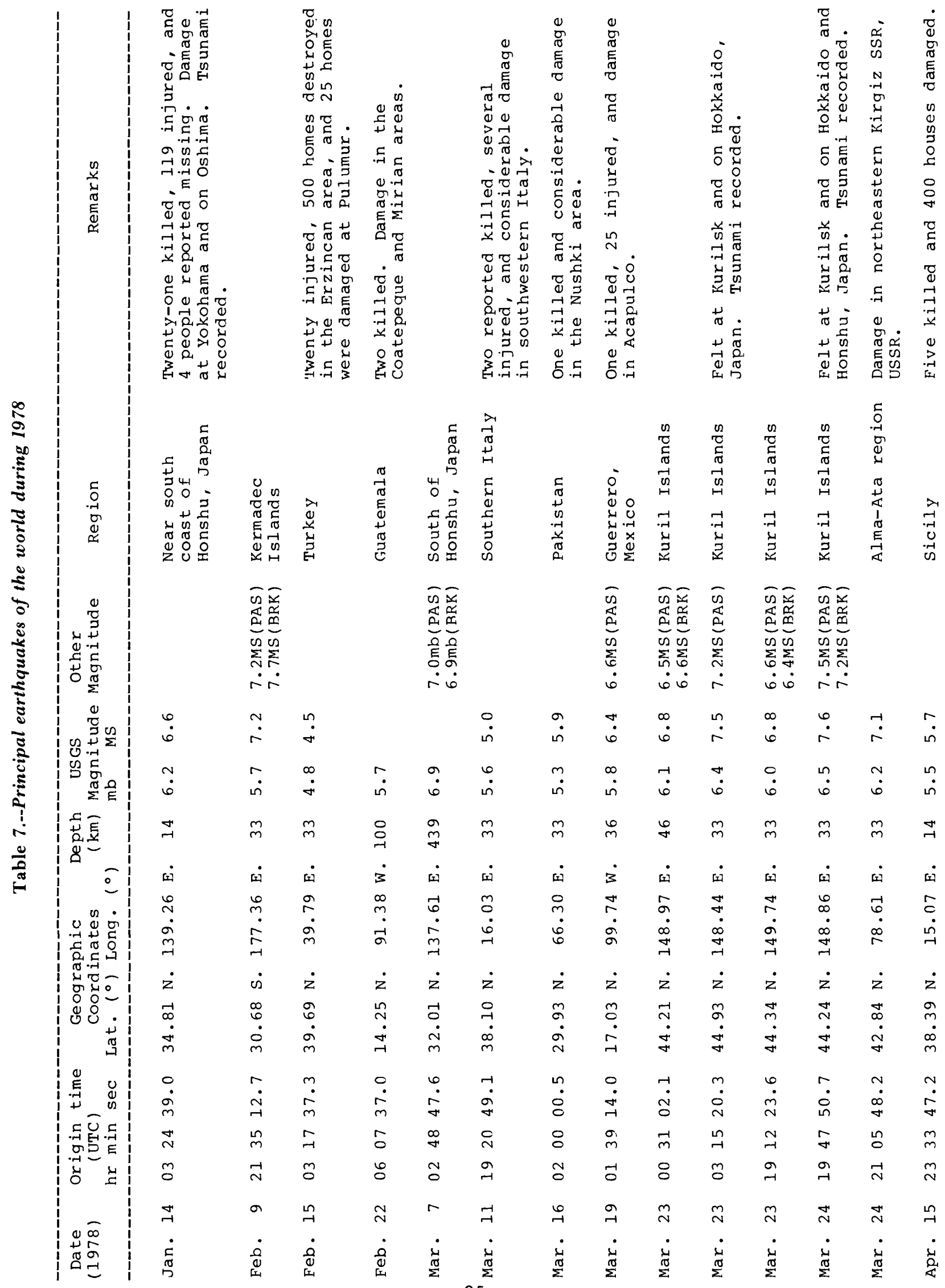




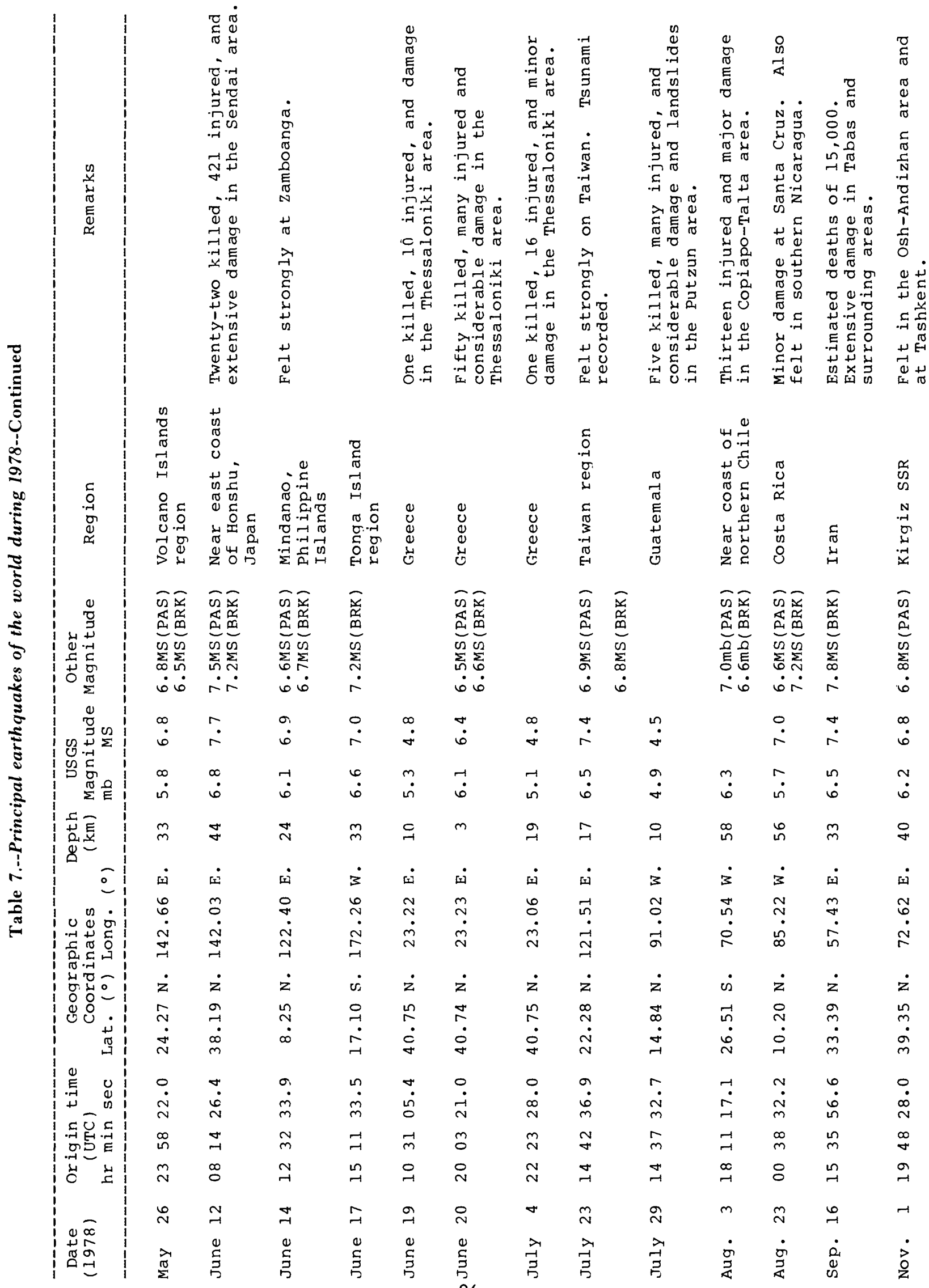




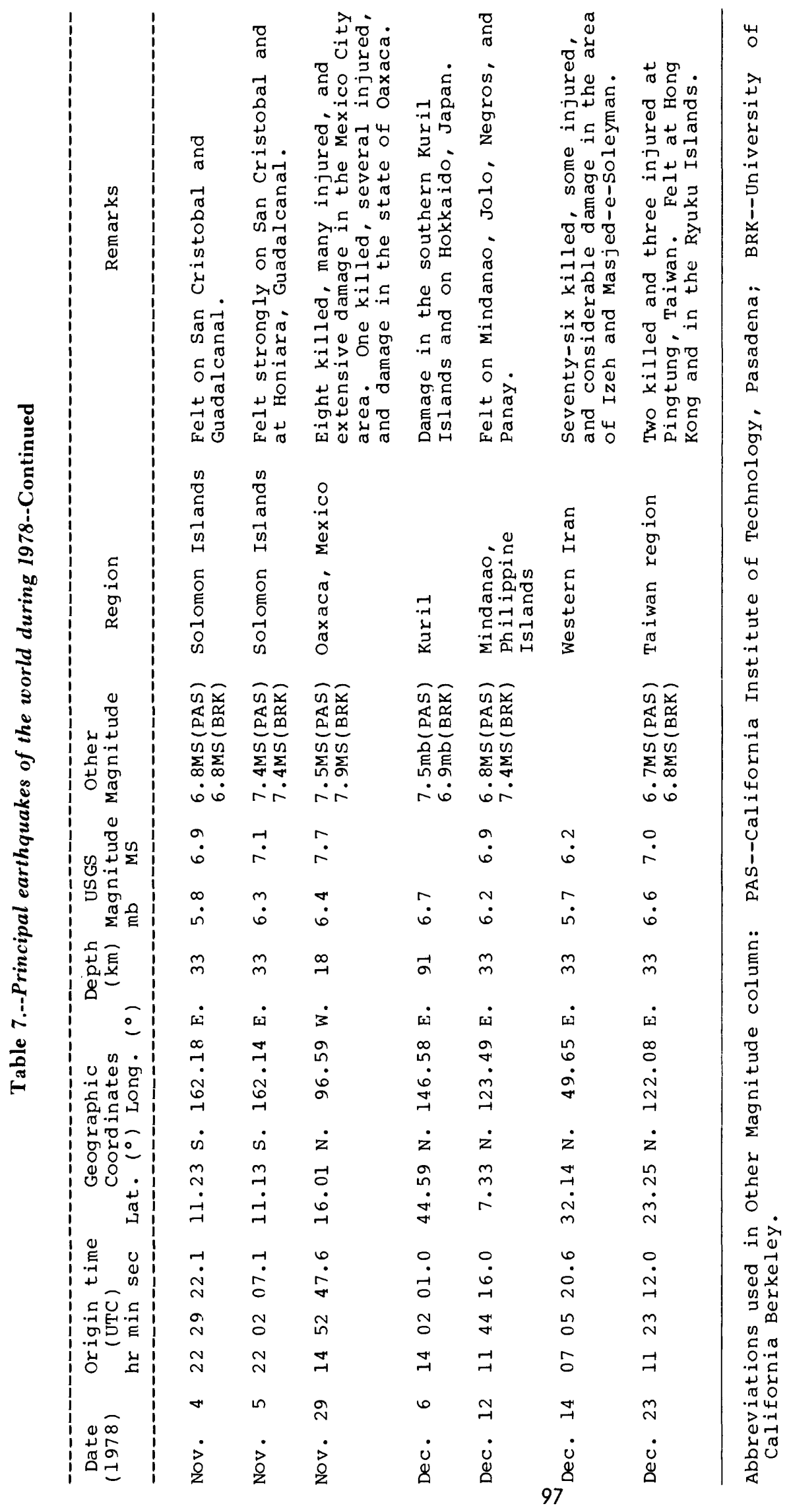




\section{Strong-Motion Seismograph Data}

\author{
By Ronald L. Porcella \\ Seismic Engineering Branch \\ U.S. Geological Survey \\ Menlo Park, California 94025
}

\section{INTRODUCTION}

The first engineering seismology program in the United States was administered by the Seismological Field Survey of the Coast and Geodetic survey (C\&GS). This program was begun in 1931 and effectively remained the responsibility of the Seismological Field survey (SFS) for more than 40 years. During this period the SFS was shifted from one acronymic agency to another, including C\&GS, ESSA, NOS, NOAA, ERL, ESL, and finally, in 1973, USGS; soon afterwards the SFS became the seismic Engineering Branch of the USGS. In spite of these numerous high-level administrative changes, the program has retained a distinct identity and its basic objectives and field-level operations have remained remarkably consonant throughout the years.

The current program of stronq-motion instrumentation administered by the USGS is supported by the National Science Foundation (Grant CA-114) in cooperation with both private industry and education institutions, as well as numerous Federal, State, and local agencies and organizations. The objectives of the program are to record strong ground motions and the response of representative types of engineered structures during potentially damaging earthquakes and to disseminate processed data and information about the records, sites, and structures to external users in earthquake engineering research and design practice and engineering seismology. The dissemination of this information and data is achieved in various ways.

Preliminary earthquake reports and a summary of recent accelerograph records are presented on a regular basis in Seismic Engineering program Reports ${ }^{1}$. These summaries include a brief description of the earthquake and strong-motion recording station, the results of routine scalings of those records that contain peak accelerations greater than $0.05 \mathrm{~g}$,

${ }^{1}$ Copies of these reports are available free on application to Branch of Distribution, U.S. Geological Survey, 1200 South Eads Street, Arlington, Va. 22202. and photographic reproductions of many of the more significant accelerograms. The program reports also contain abstracts of recent reports, notes on strong-motion information sources and the availability of digitized data, and other information pertinent to the USGS and other strongmotion programs.

Strong-Motion event and strong-motion data reports are periodically published as USGS Open-file Reports and include the results of digitization and routine analyses of strong-motion accelerograms that contain peak accelerations greater than $0.10 \mathrm{~g}$ or are related to a specific event, particular strong-motion station, or geographic group of stations. The minimum acceleration level is based primarily on the current capability of the USGS to process strong-motion records and may vary with both the degree of seismic activity and number of personnel available at any given time. Although maximum acceleration is not directly related to frequency content or duration of strong-motion, the peak acceleration $c$ an be readily obtained from an accelerogram and thus the value is commonly used as a general indicator of the potential significance of the record. Detailed information on the availability of digitized data from various sources is published regularly in Seismic Engineering Program Reports.

The Strong-Motion Accelerograph Station List is pe riodically published as a USGS Open-file Report and includes information on all of the accelerograph stations in the western hemisphere known to the USGS. Because of the ever-changing nature of this information, it is impossible to have a complete 1 ist of all of the stations in existence at any one time. Rather, the list is intended to provide that community of persons interested in strong-motion programs with a reasonably complete indication of the current status of the various strong-motion networks. Information presented in this list includes the station name and geographic coordinates, site characteristics, type and size of structure, location of instruments, and the primary sources of data. The current 1 ist contains information on approximately 1200 stations located in 38 states, Canada, the Caribbean, and throughout Central and South America.

A strong-motion information retrieval system (SMIRS) has been developed to provide up-to-date information about strong- 
which they were recorded. The system is accessible through a data terminal ( 300 baud, half duplex). The system is operational, but the information within it is incomplete and needs to be verified. A user's manual is available (Converse, 1978). To retrieve information, dial (415) 329-8600 and place the telephone handset into the terminal. When the carrier light comes on press the "line-feed" key and wait for the computer to respond (two lines will be printed); type the following:

\section{enter your name SMIRS}

Type the "enter" and "SMIRS" exactly as shown above, but replace your name with your own name. The word "enter" is five lowercase characters followed by one space; your name is typed as one continuous character string and followed by one space; and "SMIRS" is five uppercase characters. Type the carriage-return key and then the line-feed key; then you will be given instructions.

\section{ACCELEROGRAPH DATA}

Table 8 is a summary of the 95 accelerograph records recovered, although not necessarily recorded, during 1978 . This value can be compared to a yearly average of 188 records for the period 1972 to 1977 inclusive. This decrease in the reported number of accelerograms recovered in 1978 is largely due to state legislation that has given responsibility to the California Division of Mines and Geology's Office of Strong-Motion studies (OSMS) for dissemination of that organization's strong-motion data; osms data are no longer routinely 1 isted in Seismic Engineering Program Reports.

The earthquakes (table 8) are listed in chronological order and include date, time (UTC), general location, geographic coordinates, and magnitude. Information about the recording station consists of the name and location, owner, and geographic coordinates. Record data include S-wave minus trigger time and the orientation, maximum acceleration, and duration of strong-motion (greater than $0.10 \mathrm{~g}$ ) for each instrument component. Record data is included only when one or more components recorded at least $0.05 \mathrm{~g}$ at ground stations or $0.10 \mathrm{~g}$ at upper floors of buildings. The event information has been compiled principally from the Preliminary Determination of Epicenters, published by the Geological survey.

The following paragraphs summarize the results of the most significant earthquakes from which strong-motion records were obtained in 1978 .

UKIAH, CALIFORNIA - MARCH 25
March 25, at 4:27 p.m. (local time) triggered three accelerographs at Coyote Dam located $18 \mathrm{~km}$ from the epicenter (Porcella, 1978a), a U.S. Army Corps of Engineers (COE) facility approximately 175 $\mathrm{km}$ northwest of San Francisco near the town of Ukiah. The earthquake occurred off the coast of northern California at a depth of about $5 \mathrm{~km}$ and was felt in the Ukiah area; no damaqe was reported. The instrumentation at coyote Dam is owned by the COE and operated by the USGS as part of the cooperative National strong-Motion Network supported by the National Science Found ation.

Coyote Dam is an earthfill embankment approximately $1070 \mathrm{~m}$ long and $50 \mathrm{~m} \mathrm{high}$; the axis is aligned in a nearly northsouth direction. Accelerographs are located at the center crest, center toe, and south abutment, and are equipped with horizontal starters; thus, the relatively short trigger minus $s$-wave intervals (approximately $0.5 \mathrm{~s}$ ) recorded at the crest, toe, and abutment stations (fig. 39) are the result of the accelerographs trigaering on horizontal ground motion perhaps 2 or 3 sec after the first P-wave arrival. Maximum accelerations recorded at the crest, toe, and south abutment stations are $0.30 \mathrm{~g}, 0.34 \mathrm{~g}$, and $0.20 \mathrm{~g}$, respectively (fig. 39 and table 8 ).

In addition to the magnitude 4.6 earthquake, three smaller aftershocks produced minor records at coyote Dam (table 8). The maximum acceleration $(0.11 \mathrm{~g})$ was recorded on the crest instrument. Additionally, the California Division of Mines and Geology recovered several strongmotion records from stations located in the Willits-Ukiah area (Toppozada, 1978).

\section{SANTA BARBARA, CALIFORNIA - AUGUST 13}

Eight accelerograms were recovered from strong-motion stations at Santa Barbara, Goleta, Cachuma Dam, and the University of California, Santa Barbara campus (UCSB) in the days immediately following the August 13 earthquake (Porcella and others, 1978c). The main event occurred at $3: 54 \mathrm{p.m}$. (local time) and was assigned magnitudes (ML) of 5.1 (California Institute of Technology), and 5.7 (University of California, Berkeley). The hypocenter was located offshore about $6 \mathrm{~km}$ south of Santa Rarbara at a depth of $7 \mathrm{~km}$ (fig. $40)$.

Strong-motion instruments that triggered during the earthquake include triaxial accelerographs located at crest and toe sites at Cachuma Dam, a U.S. Bureau of Reclamation facility at an epicentral distance of $34 \mathrm{~km}$. A maximum horizontal ground acceleration of $0.12 \mathrm{~g}$ (fig. 4l) was recorded at the crest in a southwest direction, transverse to the axis of the embankment. A triaxial accelerograph installed at the basement level of the 

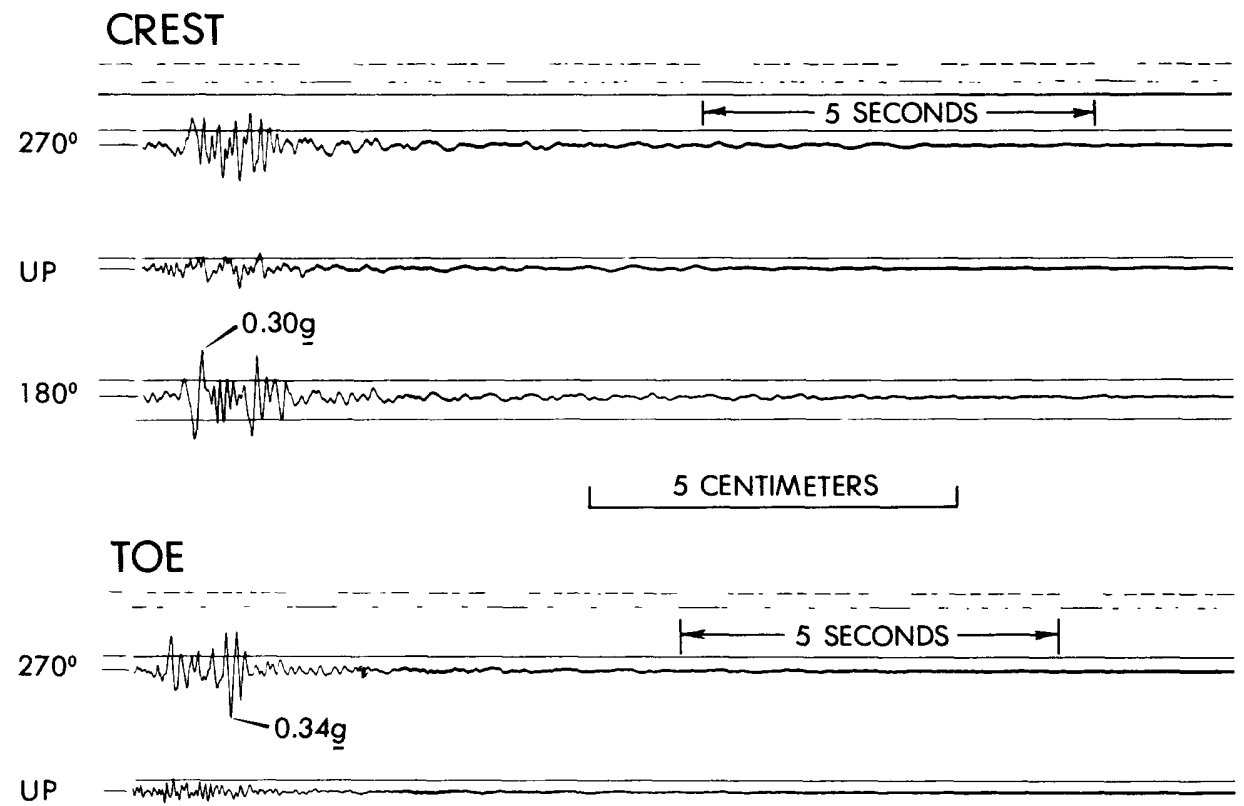

$180^{\circ}$
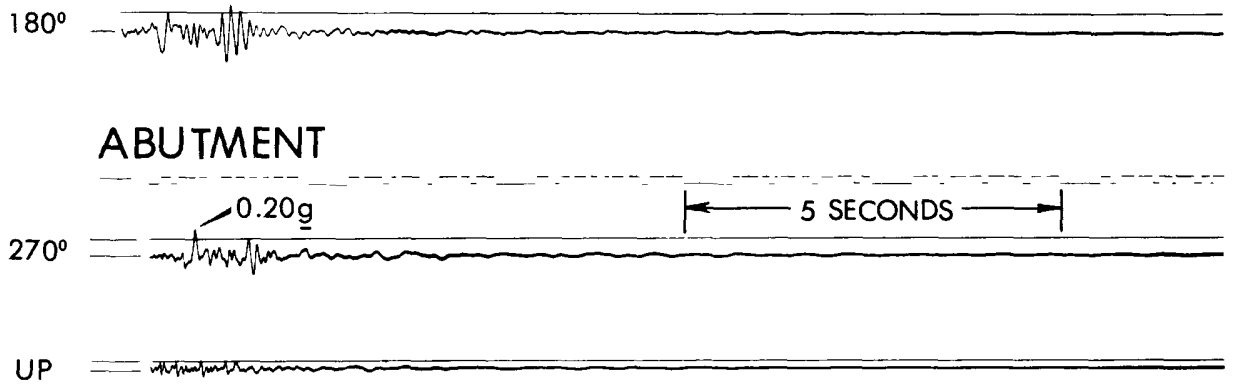

$180^{\circ}$

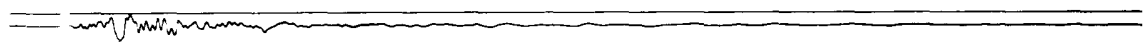

Figure 39.--Coyote Dam accelerograms from the Ukiah, California earthquake of 25 March 1978 .

Santa Barbara Courthouse recorded a maximum horizontal ground acceleration of $0.21 \mathrm{~g}$ in the southwest direction (epicentral distance $6 \mathrm{~km})$; the duration of strong-motion (greater than 0.1 g) was approximately 1.3 sec. Additionally, this instrument recorded a small aftershock about $14 \mathrm{sec}$ after triggering during the main shock (fig. 41 ).

The Southern California Edison (SCE) company recovered a strong-motion record from an SCE power facility on Glen Annie Road in Goleta; the triaxial recording system (SMA-2) employs FM modulation on a four-track magnetic tape cassette. The analog readout indicates a peak horizontal ground acceleration of $0.28 \mathrm{~g}$ (north direction). Epicentral distance $1 \mathrm{~s} 19 \mathrm{~km}$.
The California Division of Mines and Geology strong-motion program (CSMIP) includes four sites in the santa Barbara area that produced records during the August 13 event (Porter, 1978). Three of these sites are buildings instrumented by CSMIP in accordance with recently developed building instrumentation criteria. The record from a nine-channel system installed at the three-story North Hall building on the UCSB campus indicates peak horizontal accelerations of $0.44 \mathrm{~g}$, $0.66 \mathrm{~g}$, and $0.99 \mathrm{~g}$ for the ground, thi $\mathrm{rd}$ floor, and roof levels, respectively. Durations of strong (horizontal) motion (greater than $0.1 \mathrm{~g}$ ) range from $2 \mathrm{sec}$ at the ground level to approximately 9 sec at the roof level. Epicentral distance is about $13 \mathrm{~km}$. 


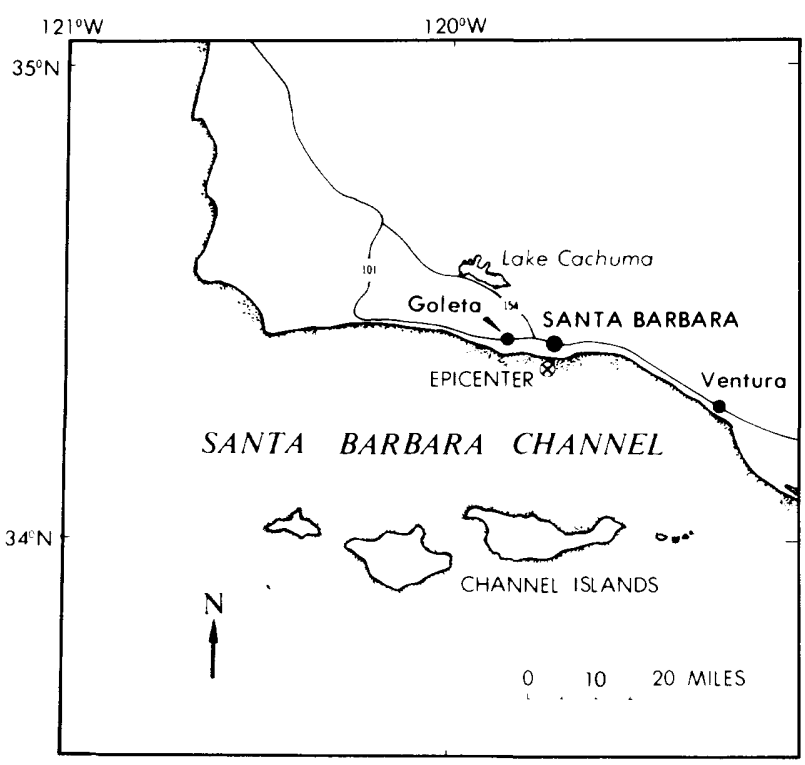

Figure 40.--Location map, Santa Barbara, California earthquake of 13 August 1978 .

The four-story Freitas Building in downtown Santa Barbara (epicentral distance $6 \mathrm{~km}$ ) is instrumented with a ninechannel remote-recording system; the strong-motion record from the August 13 event shows peak horizontal accelerations of approximately $0.22 \mathrm{~g}, 0.30 \mathrm{~g}$, and 0.67 $\mathrm{g}$ at the basement, second floor, and roof levels, respectively. Durations of strong-motion range from $0.5 \mathrm{sec}$ at the second floor to $7 \mathrm{sec}$ at the roof level.

The twelve-story Holiday Inn on Harbor Boulevard in ventura is instrumented with a twelve-channel remote-recording system; accelerometers are located at ground, fourth floor, eighth floor, and roof levels. Maximum recorded acceleration is less than $0.05 \mathrm{~g}$ at an epicentral distance of $40 \mathrm{~km}$.

A CSMIP triaxial accelerograph at the Physical plant station on the UCSB campus recorded a peak horizontal ground acceleration of about $0.37 \mathrm{~g}$ in the south direction (epicentral distance $13 \mathrm{~km}$ ). Duration of strong-motion was approxi- mately 1.5 sec. Additionally, this instrument recorded a small aftershock about 17 sec after triggering during the main event; maximum acceleration is less than $0.05 \mathrm{~g}$.

\section{JENKINSVI LLE, SOUTH CAROLINA - FEBRUARY to NOVEMBER}

Twenty-one earthquakes were recorded between February and November 1978 at Monticello Dam in north-central South Carolina; the dam and reservoir are part of the virgil C. Summer Nuclear Power Facility operated by the South Carolina Electric and Gas Company (SCEG) and are located approximately $4 \mathrm{~km}$ northwest of Jenkinsville, S.C. The reservoir covers an area of about 2750 hectares and has an average depth of about $25 \mathrm{~m}$. Filling of the reservoir began in late necember 1977 and was accompanied by an abrupt increase in seismic activity (up to 100 events per day) recorded by a network of four seismographs operated by SCEG (Porcella, 1978b). prior to filling of the reservoir, the USGS seismograph station at Jenkinsville had recorded one event about every six days. This increase in activity tapered off and then resumed abruptly in August 1978. Two strong-motion accelerographs were installed by the USGS in February 1978 as a result of the first increase in seismic activity. One accelerograph was placed in an office building a few hundred meters west of the power plant; the other instrument was installed on the south abutment of the largest of four embankment sections. Thirteen records with peak accelerations greater than $0.05 \mathrm{~g}$ have been recovered from these two stations (table 8 ). The largest acceleration was recorded at the abutment site and suggests a $0.253 \mathrm{~g}$ peak horizontal ground acceleration in the "south" direction (fig. 42); the duration of strong-motion (greater than $0.1 \mathrm{~g})$ is about 0.06 sec at approximately $25 \mathrm{~Hz}$. This record has been attributed to a magnitude 2.7 event (depth $1.5 \mathrm{~km}$ ) on August 27, 1978 at 10:23:08 UTC and contains the largest known recorded acceleration from an earthquake in central or eastern North America. Epicentral distance is about $1 \mathrm{~km}$. The absence of trigger minus $S$ or S-P intervals on any of the 21 records indicates sources very near the recording stations. 

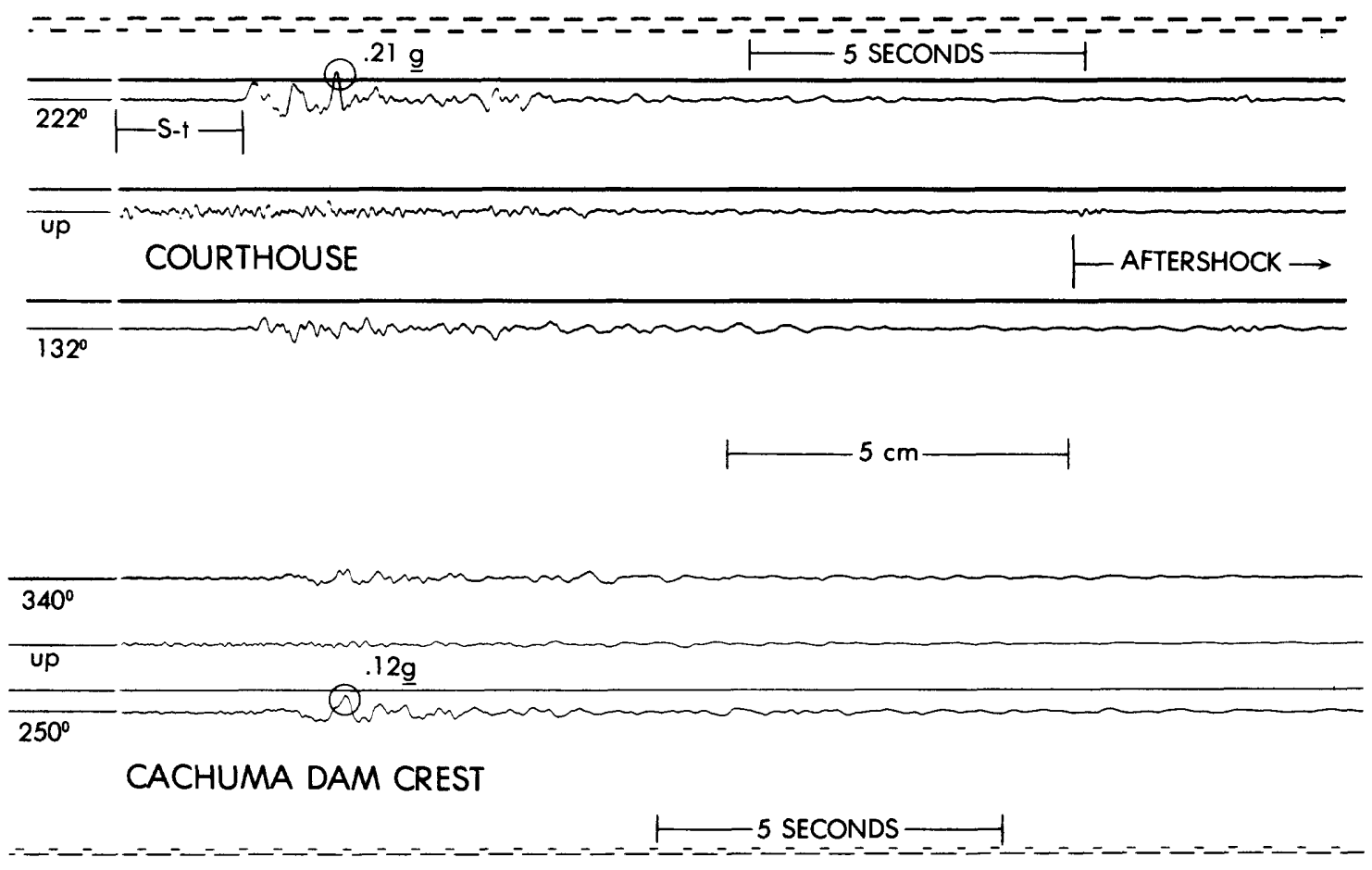

\section{CACHUMA DAM TOE}

$340^{\circ}$

$\frac{\text { up }}{250^{\circ}} \longrightarrow 07 \mathrm{~g}$

Figure 41.--Accelerograms from Santa Barbara Courthouse and Cachuma Dam, Santa Barbara earthquake of 13 August 1978. Component direction refers to direction of case acceleration for upward trace deflection on accelerogram. 
27 August, 1023 UTC
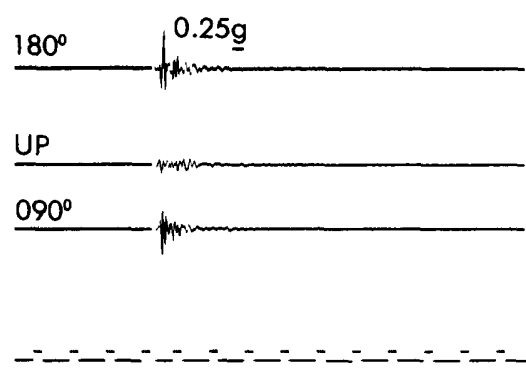

5 SECONDS

31 August - 6 November
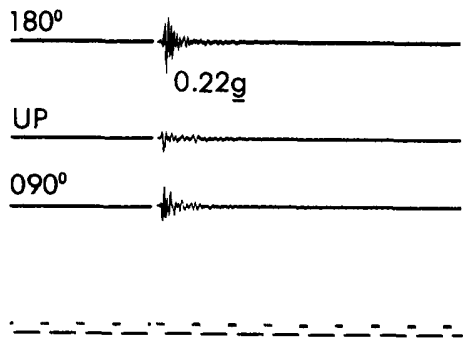

$27-31$ August

$180^{\circ}$

$\underline{U P}$

$090^{\circ}$
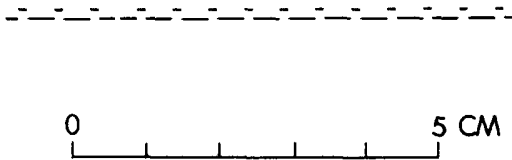

31 August - 6 November

$180^{\circ}$
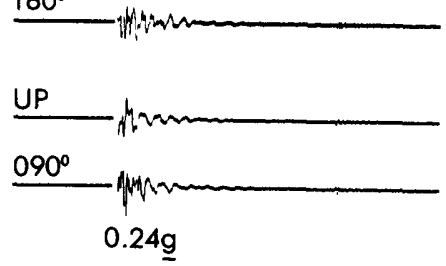

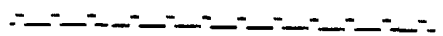

Figure 42.--Selection of Monticello Dam accelerograms from the Jenkinsville, South Carolina earthquakes of February to November 1978. 
Table 8.--Summary of U.S. accelerograph records recovered during 1978

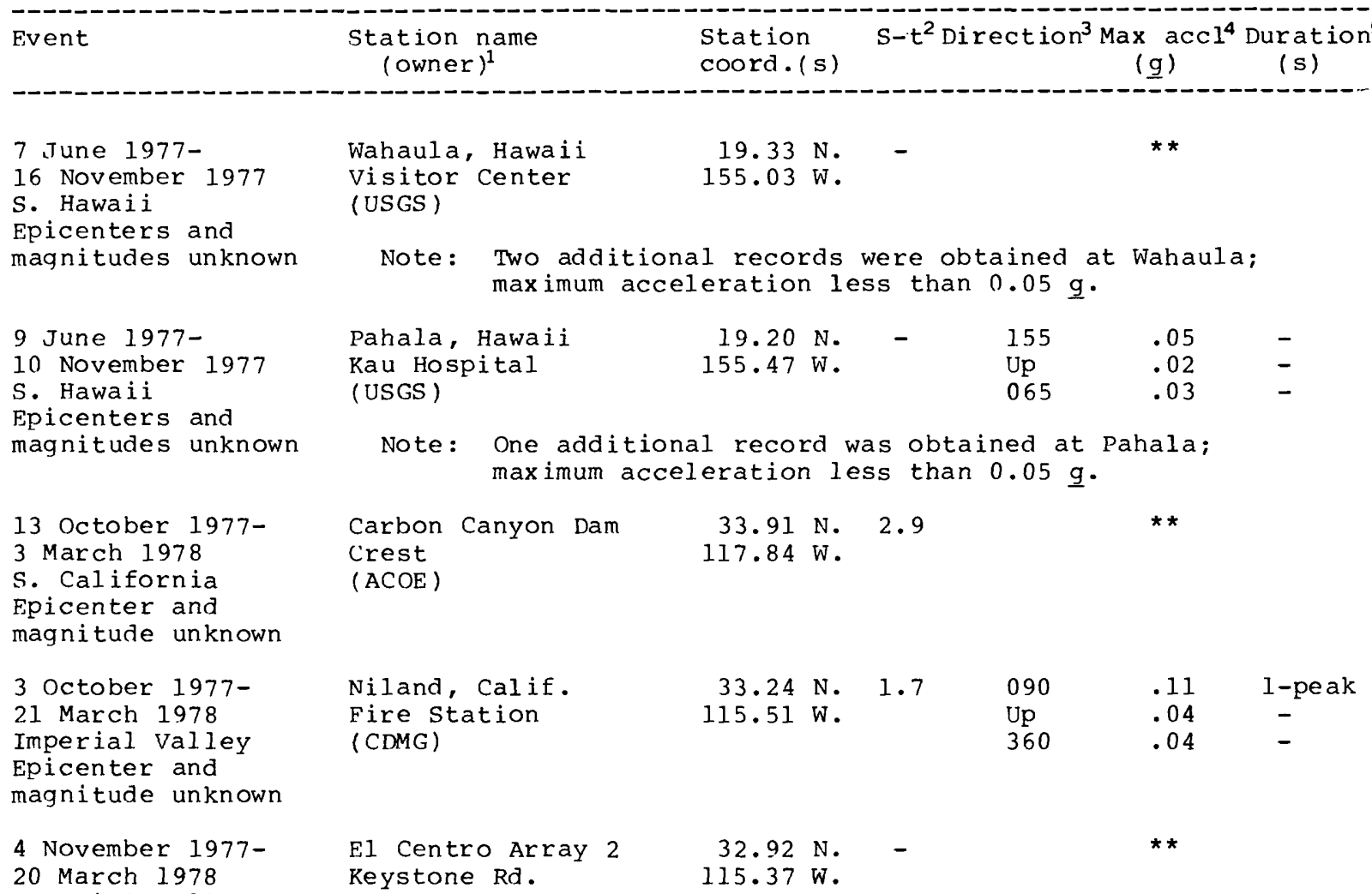
Imperial Valley Epicenters and magnitudes unknown

14 November 197720 March 1978 Imperial Valley Epicenters and magnitudes unknown

15 November 1977

1913 UTC

Imperial Valley

$32.83 \mathrm{~N}$. , $115.47 \mathrm{~W}$.

Magnitude 3.4

15 November 1977

2200 UTC

Imperial Valley

Epicenter and

magnitude unknown

15 November 1977-

23 March 1978

Imperial Valley

Epicenter and

magnitude unknown

\section{(USGS)}

$115.37 \mathrm{~W}$.

Note: One additional record was obtained at Array 2; maximum acceleration less than $0.05 \mathrm{~g}$.
E1 Centro Array 6 Huston Rd. ( CDMG)

\begin{tabular}{|c|c|c|c|c|}
\hline $\begin{array}{r}32.84 \mathrm{~N} . \\
115.49 \mathrm{~W} .\end{array}$ & 2.2 & $\begin{array}{l}230 \\
\text { Up } \\
140\end{array}$ & $\begin{array}{l}.14 \\
. \\
.21\end{array}$ & $\begin{array}{l}1-\text { peak } \\
- \\
0.3\end{array}$ \\
\hline & 2.1 & $\begin{array}{l}230 \\
\text { Up } \\
140\end{array}$ & $\begin{array}{c}.07 \\
-17\end{array}$ & $\begin{array}{l}- \\
\text { 1-peak }\end{array}$ \\
\hline & 2.1 & & $\pi \pi$ & \\
\hline & 2.0 & $\begin{array}{l}230 \\
\text { Up } \\
140\end{array}$ & $\begin{array}{c}.04 \\
.06\end{array}$ & - \\
\hline
\end{tabular}

El Centro Array 7 Imp. Valley college (USGS )

E1 Centro Array 7 Imp. Valley College (USGS)
32.83 N. 1.1

$115.50 \mathrm{~W}$.
32.83 N. 1.6 $115.50 \mathrm{~W}$.
230

Up

140
.05

.03

.06
-

-
E1 Centro Array 9 Commercial Ave. (USGS)
$32.79 \mathrm{~N}$. $115.55 \mathrm{~W}$. 
Table 8.--Summary of U.S. accelerograph records recovered during 1978--Continued

\begin{tabular}{|c|c|c|c|c|c|c|}
\hline Event & $\begin{array}{l}\text { Station name } \\
\text { (owner) }\end{array}$ & $\begin{array}{l}\text { Station } \\
\text { coord. (s) }\end{array}$ & & ectic & (g) & $\begin{array}{l}\text { Durat } \\
\text { (s) }\end{array}$ \\
\hline $\begin{array}{l}11 \text { December } 1977 \\
0557 \text { UTC } \\
\text { Imperial Valley } \\
32.57 \mathrm{~N} \text {. } 115.32 \mathrm{~W} \text {. } \\
\text { Magnitude } 3.4\end{array}$ & $\begin{array}{l}\text { Bonds Corner, Calif. } \\
\text { Ground Level } \\
\text { (USGS) }\end{array}$ & $\begin{array}{r}32.693 \mathrm{~N} . \\
115.338 \mathrm{~W} .\end{array}$ & - & & $\star \star$ & \\
\hline \multirow{3}{*}{$\begin{array}{l}26 \text { March } 1978 \\
\text { 0027 UTC } \\
\text { N. California } \\
\text { 39.09N. } 123.34 \mathrm{~W} \text {. } \\
\text { Magnitude } 4.5\end{array}$} & $\begin{array}{l}\text { Ukiah, Calif. } \\
\text { Coyote Dam, Crest } \\
(A C O E)+\end{array}$ & $\begin{array}{r}39.20 \mathrm{~N} . \\
123.18 \mathrm{~W} .\end{array}$ & - & $\begin{array}{l}270 \\
\mathrm{Up} \\
180\end{array}$ & $\begin{array}{l}.25 \\
.14 \\
.30\end{array}$ & $\begin{array}{l}1 \cdot 0 \\
0.3 \\
1.3\end{array}$ \\
\hline & $\begin{array}{l}\text { Ukiah, Calif. } \\
\text { Coyote Dam, Toe } \\
(\text { ACOE)+ }\end{array}$ & $\begin{array}{r}39.20 \mathrm{~N} . \\
123.18 \mathrm{~W} .\end{array}$ & - & $\begin{array}{l}270 \\
\mathrm{Up} \\
180\end{array}$ & $\begin{array}{l}.34 \\
.09 \\
.22\end{array}$ & $\begin{array}{l}1.1 \\
- \\
1.2\end{array}$ \\
\hline & $\begin{array}{l}\text { Ukiah, Calif. } \\
\text { Coyote Dam, Abutment } \\
(\text { ACOE })+\end{array}$ & $\begin{array}{r}39.19 \mathrm{~N} . \\
123.18 \mathrm{~W} .\end{array}$ & - & $\begin{array}{l}270 \\
\text { Up } \\
180\end{array}$ & $\begin{array}{l}.20 \\
.07 \\
.11\end{array}$ & $\begin{array}{l}0.8 \\
- \\
1-\text { pe }\end{array}$ \\
\hline $\begin{array}{l}26 \text { March } 1978- \\
27 \text { March } 1978 \\
\text { N. California }\end{array}$ & $\begin{array}{l}\text { Ukiah, Calif. } \\
\text { Coyote Dam, Crest } \\
\text { (ACOE) }\end{array}$ & $\begin{array}{r}39.20 \mathrm{~N} . \\
123.18 \mathrm{~W} .\end{array}$ & - & $\begin{array}{l}270 \\
\text { Up } \\
180\end{array}$ & $\begin{array}{l}.11 \\
.04 \\
.07\end{array}$ & $\begin{array}{l}1-p e \\
- \\
-\end{array}$ \\
\hline
\end{tabular}

Epicenters and

magnitudes unknown

25 March 1977

1544 UTC

Imperial Valley

$32.97 \mathrm{~N}$. , 115.50W.

Magnitude 3.4

21 October 1977

0612 UTC

Imperial Valley

$32.90 \mathrm{~N}, 115.50 \mathrm{~W}$.

Magnitude 4.3

24 February 1978-

16 March 1978

Jenkinsville, S.C. Epicenters and

magnitudes unknown

Note: Two additional aftershocks recorded at crest station and three aftershocks each recorded at abutment and toe stations. Maximum acceleration less than $0.05 \mathrm{~g}$.

Brawley Airport Ground Level (USGS)

$32.99 \mathrm{~N} \cdot 1.8$ $115.51 \mathrm{~W}$.

1.8

315

UP

225

$32.99 \mathrm{~N}$.

$115.51 \mathrm{~W}$. Ground Level (USGS)

Jenkinsville, S.C. Monticello Dam (USGS)

Abutment (Office)

Shared abutment (Center crest)
16 March 1978-

3 May 1978

Jenkinsville, S.C.

Epicenters and

magnitudes unknown
Jenkinsville, S.C. Monticello Dam (USGS) Shared abutment (Center crest)
$34.30 \mathrm{~N}$. $81.33 \mathrm{~W}$.

\begin{tabular}{|c|c|c|c|}
\hline- & $\begin{array}{l}090 \\
\text { Up } \\
360\end{array}$ & $\begin{array}{r}.05 \\
.03 \\
.03\end{array}$ & $\begin{array}{l}- \\
-\end{array}$ \\
\hline- & & $\star \star$ & \\
\hline - & & $\pi \star$ & \\
\hline- & $\begin{array}{l}180 \\
\text { Up } \\
090\end{array}$ & $\begin{array}{l}.15 \\
.10 \\
.07\end{array}$ & $\begin{array}{c}1 \text {-peak } \\
1 \text {-peak } \\
\text { - }\end{array}$ \\
\hline - & $\begin{array}{l}180 \\
\text { Up } \\
090\end{array}$ & $\begin{array}{l}.06 \\
.06 \\
.07\end{array}$ & $\begin{array}{l}- \\
-\end{array}$ \\
\hline- & & $\begin{array}{l}\star \star \\
\star \star\end{array}$ & \\
\hline
\end{tabular}

$34.30 \mathrm{~N}$ $81.33 \mathrm{~W}$. 
Table 8.--Summary of U.S. accelerograph records recovered during 1978--Continued

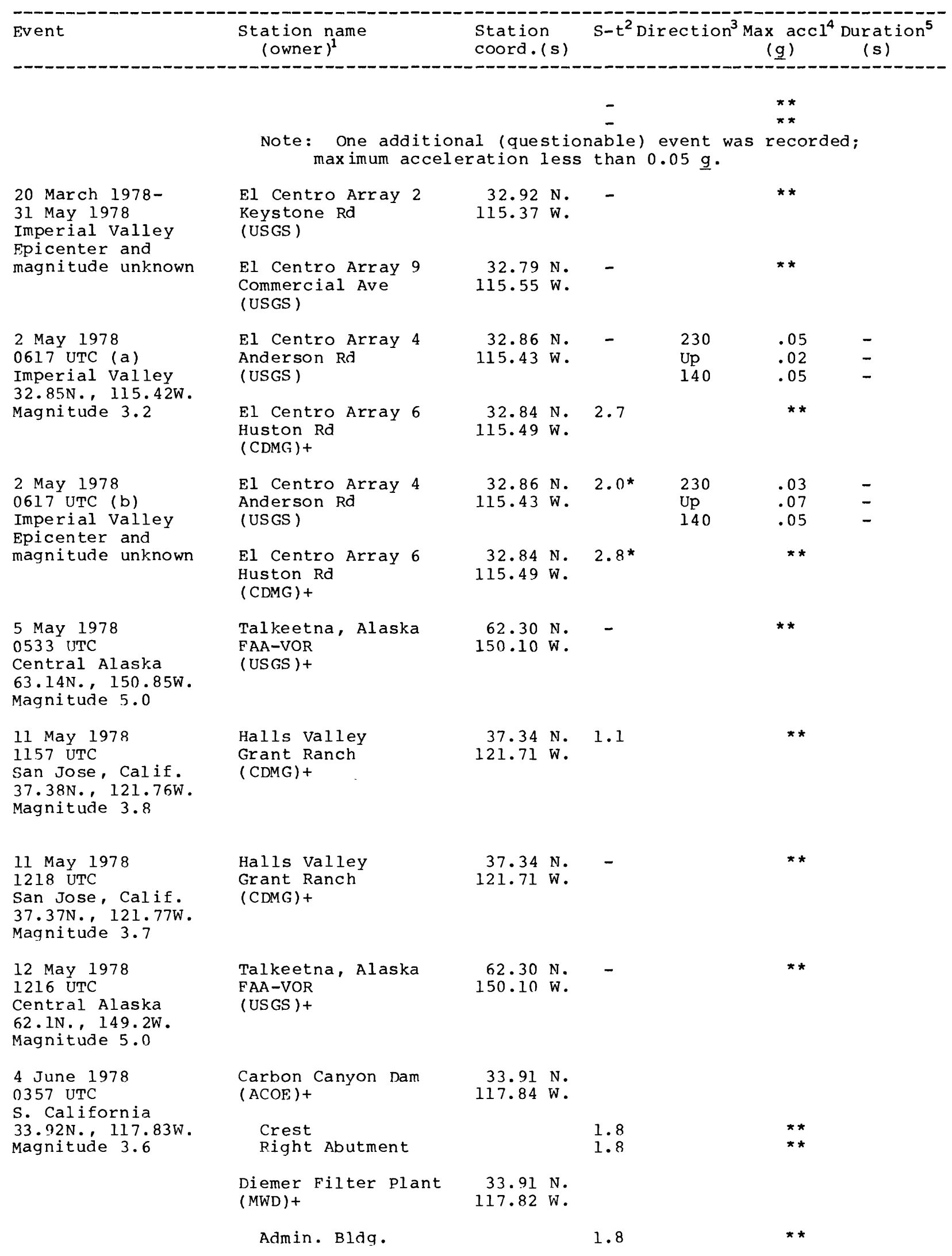


Table 8.--Summary of U.S. accelerograph records recovered during 1978--Continued

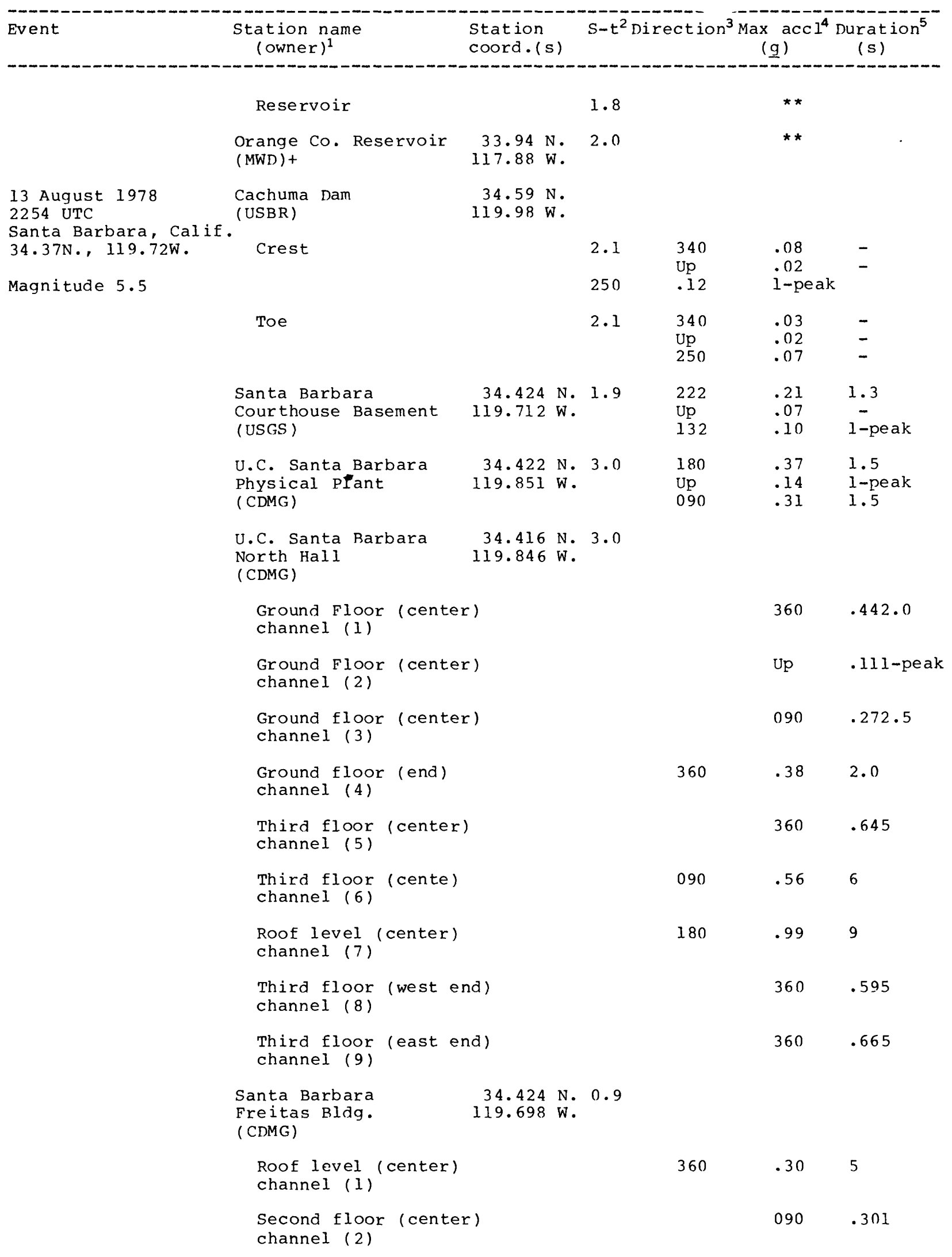


Table 8.--Summary of U.S. accelerograph records recovered during 1978--Continued

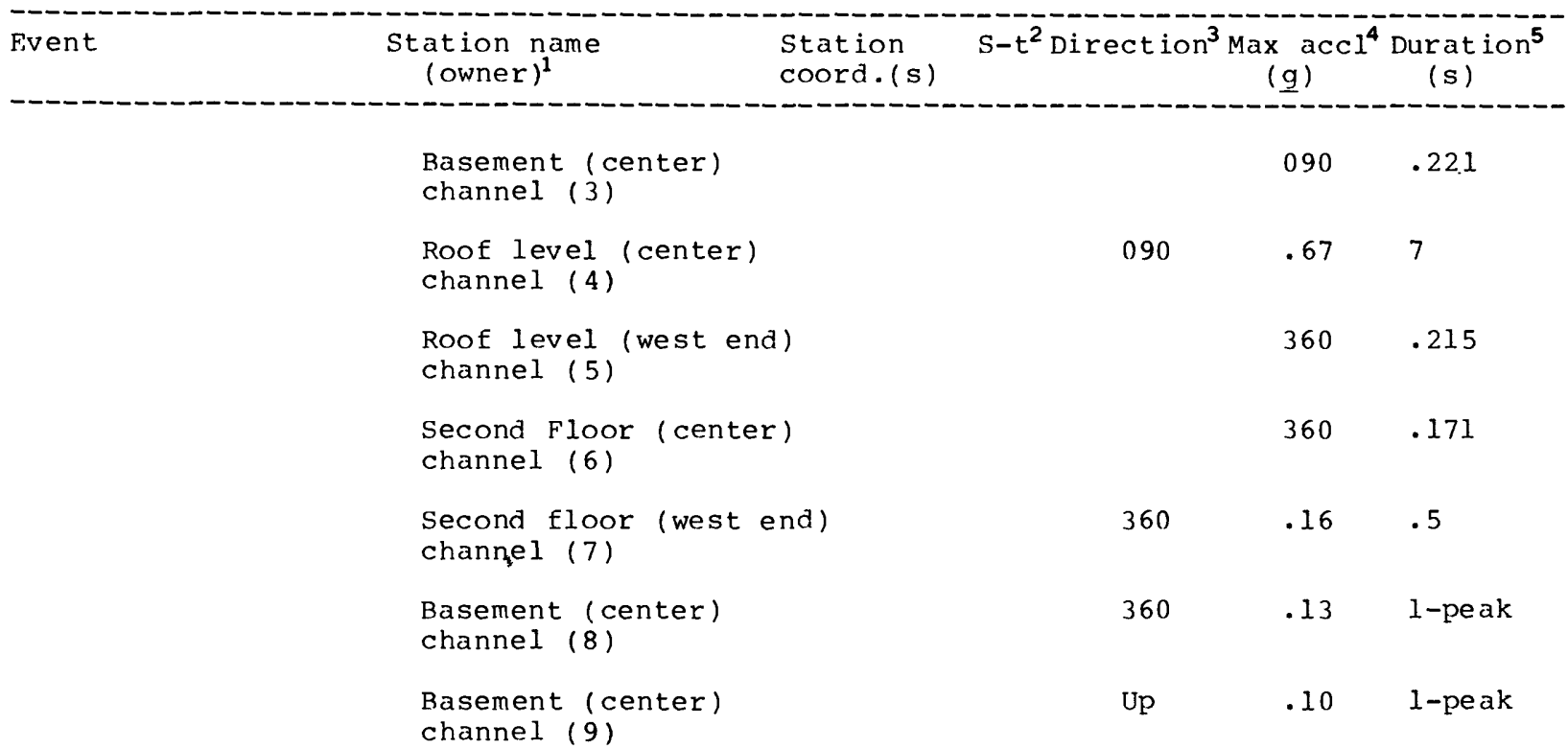

Note: Acceleration and duration-data for UCSB (Physical plant and North Hall) and Goleta (Freitas Bldg.) are approximations.

Ventura, Calif. Holiday Inn ( CDMG)
$34.276 \mathrm{~N}$. $119.294 \mathrm{~W}$.

Note: Building is 12 stories above ground level and is instrumented with a 12-channel CRA-1 remote recording system. Accelerometers are located at ground, 4 th floor, 8 th floor, and roof levels. A vertical starter is located at ground level; a horizontal starter is located at the roof level.

\begin{tabular}{|c|c|c|c|}
\hline $\begin{array}{l}\text { Goleta, Calif. } \\
\text { Glen Annie Rd } \\
\text { (SCE) }\end{array}$ & $\begin{array}{r}34.47 \mathrm{~N} . \\
119.89 \mathrm{~W} .\end{array}$ & 2.4 & $\begin{array}{l}090 \\
\text { Up } \\
360\end{array}$ \\
\hline
\end{tabular}

Note: A small aftershock was recorded at U.C. Santa Barbara Physical Plant and Santa Barbara Courthouse. Maximum acceleration less than $0.05 \mathrm{~g}$. Two seismoscope records were recovered at UCSB and Santa Barbara Courthouse; maximum relative displacements are 4.06 and $3.42 \mathrm{~cm}$, respectively.

27 August 1978

1023 UTC

Jenkinsville, S.C. $34.31 \mathrm{~N}, 81.33 \mathrm{~W}$. Magnitude 2.7
Jenkinsville, S.C. Monticello Dam (USGS)+

Shared abutment (Center crest)
$34.30 \mathrm{~N}$.

$81.33 \mathrm{~W}$.

$\begin{array}{cccc}- & 180 & .25 & .06 \\ \text { Up } & .08 & - \\ 090 & .20 & .16 \\ - & & \star \star & \\ - & & & \\ & 180 & .08 & - \\ \text { Up } & .04 & - \\ 090 & .06 & -\end{array}$

31 August 19786 November 1978 Jenkinsville, S.C. Epicenters and
Jenkinsville, S.C. $\quad 34.30 \mathrm{~N}$. Monticello Dam $81.33 \mathrm{~W}$. (USGS) 
Table 8.--Summary of U.S. accelerograph records recovered during 1978--Continued

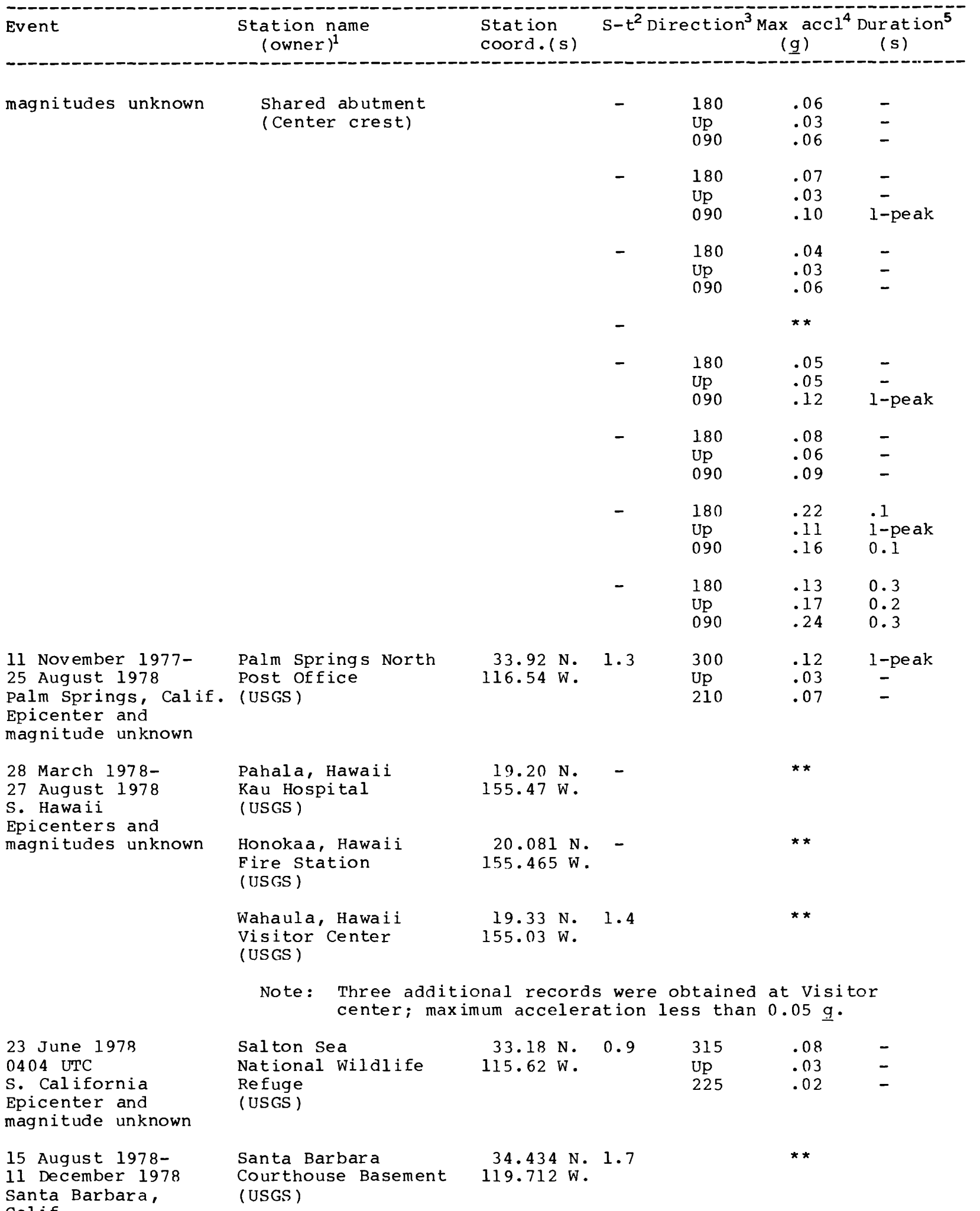

\section{Calif.}


Table 8.--Summary of U.S. accelerograph records recovered during 1978--Continued

Pine Flat Dam
$\begin{aligned} & \text { Piedra, Calif. } \\ & \text { (ACOE)+ }\end{aligned}$

${ }^{1}$ ACOE - U.S. Army Corps of Engineers

CDMG - California Division of Mines and Geology

MWD - Metropolitan Water District

SCE - Southern California Edison

USBR - U.S. Bureau of Reclamation

USGS - U.S. Geological Survey

+ - WWVB time code is incomplete or nonexistent; correlation of accelerogram with event is questionable.

${ }^{2} \mathrm{~S}$-wave minus trigger time.

* Denotes S-P interval, that is, the earthquake occurred within the instrumental run-time of a previous event.

${ }^{3} \mathrm{Az}$ imuthal direction of case acceleration for upward trace deflection on accelerogram (opposite direction to pendulum motion). Case acceleration for vertical component indicated as up or down.

${ }^{4}$ Unless otherwise noted, maximum acceleration recorded at ground or basement 1 evel. ** Denotes maximum acceleration is less than $0.05 \mathrm{~g}$ at ground stations or less than $0.10 \mathrm{~g}$ at upper floors of buildings.

${ }^{5}$ Duration for which peaks of acceleration exceed $0.10 \mathrm{~g}$. 


\section{References}

Bath, Markus, 1966, Earthquake energy and magnitude, in Physics and Chemistry of the Earth, Volume 7: Oxford and New York, Pergamon Press, p. 115-165.

Bennett, J. H., Sherburne, R. W. , Cramer, C. H., Chesterman, C. W., and Chapman, R. H., 1979, Stephens Pass earthquakes: California Geology, $v$. 32 , no. 2 , p. 27-34.

Converse, A., 1978, Strong-motion information retrieval system user's manual: U.S. Geological Survey Open-file Report 79-289, $51 \mathrm{p}$.

DuBois, S. M., and Wilson, F. W., 1978, List of earthquake intensities for Kansas, 1867-1977: Kansas Geological Survey, Environmental Geology Series $2,56 \mathrm{p}$.

Gutenberg, B. and Richter, C. F., 1956, Magnitude and energy of earthquakes: Annali di Geofisca, v. 9, no. 1 , p. $1-15$.

Johnson, C. E., 1979, I. CEDAR - An approach to computer automation of short-period local seismic networks, Ph.D. Thesis, Division of Geological and Planetary Sciences, California Institute of Technology.

Lawson, J. E., Jr., 1978, A preliminary duration magnitude scale for local and regional earthquakes recorded at oklahoma seismograph stations: Oklahoma Geophysical Observatory Open-file Report, $14 \mathrm{p}$.

Lee, W. H. K., Johnson, C. E. , Heneyey, T. L., and Yerkes, R. C., 1978, A preIiminary study of the Santa Barbara, California earthquake of August 13, 1978 and its major aftershocks: U.S. Geological Survey Circular 797, 11 p.

Luza, K. V., 1978, Regional seismic and geologic evaluations of Nemaha Uplift: Oklahoma, Kansas, and Nebraska, Oklahoma Geology Notes, $v$. 38 , p. 49-58.

McKenzie, M. R. , Miller, R. D., and Uhrhammer, R. A., 1978, Bulletin of the Seismographic Stations, v. 48, nos. 1 and $2,83 \mathrm{p} .$, January 1 , 1978December 31 , 1978 .

Merriam, D. F., 1956, History of earthquakes in Kansas: Bulletin of the Seismological Society of America, $v$. 46 , no. 2 , p. $87-96$
Miller, R. K. and Felszeghy, S. F., 1978, Engineering features of the Santa Barbara earthquake of August 13, 1978: Earthquake Engineering Research Institute and University of California, Santa Barbara, UCSB-ME$78-2,140 \mathrm{p}$.

Nicholson, C. and Singh, S., 1978, A detailed microearthquake study of the New Madrid Fault Systems western Tennessee segment (abstract): Earthquake Notes, v. 49, p. 19.

Nuttli, o. W. 1973, Seismic wave attenuation and magnitude relations for eastern North America: Journal of Geophysical Research, v. 78, no. 5, p. 876-885.

Porcella, R. L., 1978a, Recent strongmotion records, in Seismic Engineering program Report, January - April 1978: Geological Survey Circular 785-A, p. 1-2.

Porcella, R. L., 1978b, Recent strongmotion records, in Seismic Engineering Program Report, May - August 1978: Geological Survey Circular $785-\mathrm{C}, \mathrm{p} .1$.

Porcella, R. L., 1978c, Recent strongmotion records, in Seismic Engineering program Report, September December 1978: Geological Survey Circular 785-C, p. 1 .

Porcella, R. L., Maley, R. P., and Acosta, A. V., 1978, Strong-motion results from the Santa Barbara, California earthquake of August 13, 1978, in Seismic Engineering Program Report, May - August 1978: Geological survey Circular 785-B, p. 8-10.

Porter, L. D., 1978, Compilation of strong-motion records recovered from the Santa Barbara earthquake of 13 August 1978: California Division of Mines and Geology, Preliminary Report $22,43 \mathrm{p}$.

Richter, C. F., 1958, Elementary Seismology: San Francisco, Calif., W. H. Freeman and Co., Inc., $768 \mathrm{p}$.

Stanley, K. O., and Wayne, W. J., 1972, Epeirogenic and climatic controls of early pleistocene fluvial sediment dispersal in Nebraska: Bulletin of the Geological Society of America v. 83, p. $3675-3690$ 
Toppozada, T. R., 1978, Earthquakes in the Willits-Ukiah area: California Division of Mines and Geology, California Geology, June 1978, p. 146147.

Whi tcomb, J. H., Allen, C. R., Blanchard, A. C., Fisher, S. A., Fuis, G. S., Hutton, L. K., Jenkins, D. J., Johnson, C. E., Reed, B. A., Richter K. J., 1978, Southern California Array for Research on Local Earthquakes and Teleseisms (SCARLET) Caltech - USGS Monthly preliminary Epicenters for January 1977 to March 1978: Seismological Laboratory, Division of Geological and planetary sciences, California Institute of Technology.

Whi tcomb, J. H., Allen, C. R., Blanchard, A. C., Fisher, S. A., Fuis, G. S., German, P. T., Hutton, I. K., Johnson, C. E., Lamanuzzi, V. D., Reed, B. A., Richter, K. J., 1979, Southern California Array for Research on
Local Earthquakes and Teleseisms (SCARLET) Caltech - USGS Monthly Epicenters from March 1978 to January 1979: Seismological Laboratory, Division of Geological and Planetary Sciences, California Institute of Technology.

Wood, H. O., and Neumann, F., 1931, Modified Mercalli Intensity Scale of 1931: Bulletin of the Seismological Society of America, v. 21 , no. 4, p. $277-283$.

woollard, G. P., 1958, Areas of tectonic activity as indicated by earthquake epicenters: American Geophysical Union. Trans., v. 39, p. 1135-1150.

Zollweg, James, 1974, A preliminary study of the seismicity of the central United States: St. Louis University unpubl ished undergraduate report, 15 p. 
NGSDC PUBLICATIONS (SOLID EARTH)

The publications 1 isted below are available from the National Geophysical and Solar-Terrestrial Data Center, NOAA/EDIS, (D61), 325 Broadway, Boulder, CO 80303 . Please allow 4 weeks for receipt of the reports. Minimum charge per order is $\$ 3$.

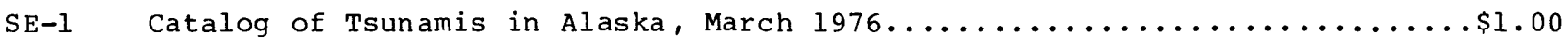

$\mathrm{SE}-2$

$\mathrm{SE}-3$

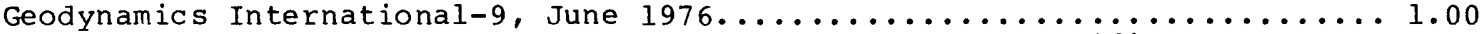

Summary of earthquake Focal Mechanisms for the Western Pacific -

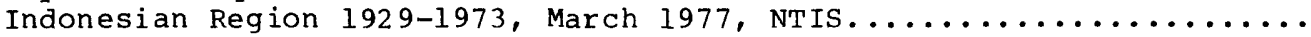

$\mathrm{SE}-4$

$\mathrm{SE}-5$

$\mathrm{SE}-6$

$\mathrm{SE}-7$

$\mathrm{SE}-8$

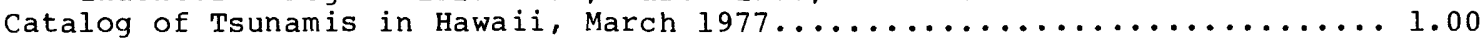

Geodynamics International-10, April $1977 \ldots \ldots \ldots \ldots \ldots \ldots \ldots$

Catalog of Seismograms and Strong-Motion Records, May 1977........... 2.00

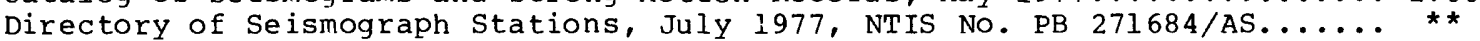

Survey of Practice in Determining Magnitudes of Near Earthquakes, Part 2, Europe, Asia, Africa, Australasia, the Pacific, August 1977, NTIS No.

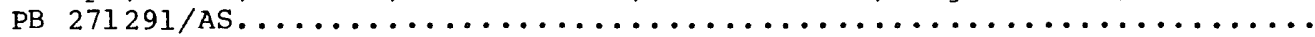

SE-9 Survey of Practice in Determining Magnitudes of Near Earthquakes, Part 1,

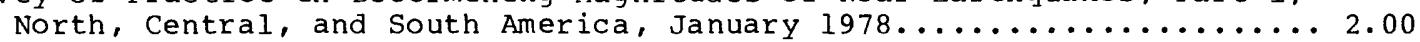

$\mathrm{SE}-10$

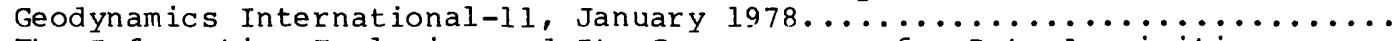

The Information Explosion and Its Consequences for Data Acquisition

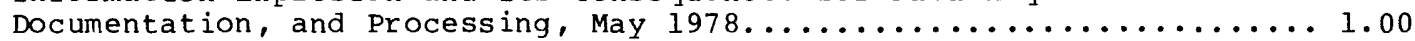

$\mathrm{SE}-12$

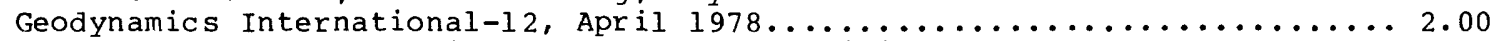

SE-13 Bibliography of Statistical Aspects of Seismicity, August 1978......... 2...0

SE-14 Directory of U.S. Data Repositories Supporting the International

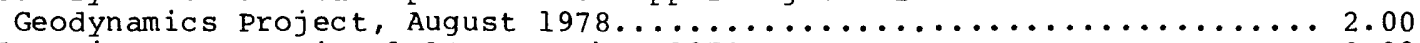

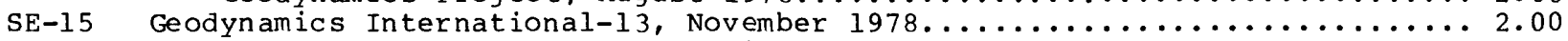

$\mathrm{SE}-16$

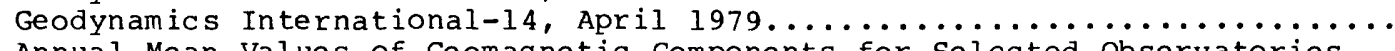

Annual Mean Values of Geomagnetic Components for Selected observatories,

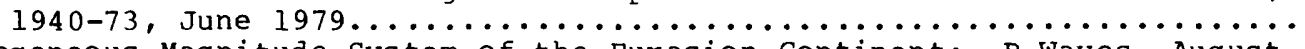

2. 00

$\mathrm{SE}-17$

$\mathrm{SE}-18$

Homogeneous Magnitude System of the Eurasion Continent: $P$ Waves, August

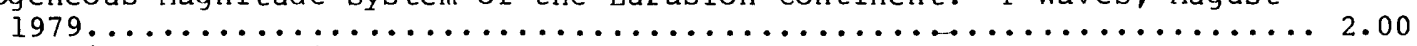

$\mathrm{SE}-19$

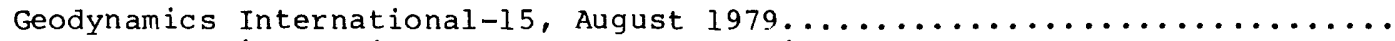

Manual of Seismological Observatory Practice, September 1979, NTIS

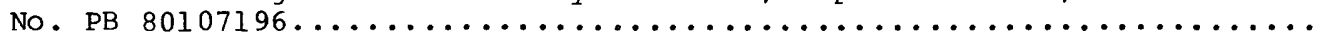

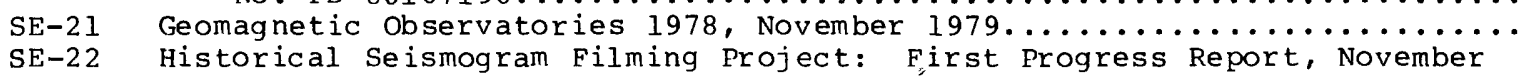

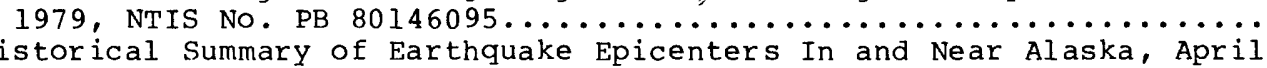
NGSDC-1 A Historical Summary of Earthquake Epicenters In and Near Alaska, April

NGSDC-2 Final Report: An Analysis of Earthquake Intensities with Respect to Attenuation, Magnitude and Distance, August $1976 \ldots \ldots \ldots \ldots \ldots \ldots$

2.00
2.00

NGSDC-3 An Analysis of Earthquake Intensities and Recurrence Rates In and Near

3.00

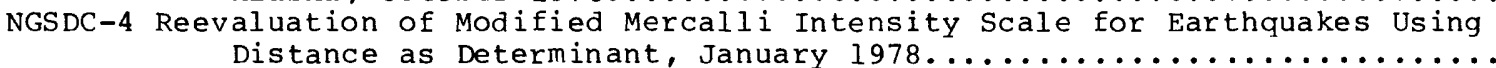

NGSDC Data Fact Sheet No. 1. Some Summary Geomagnetic Activity Data $1932-1976$

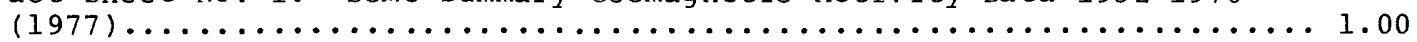

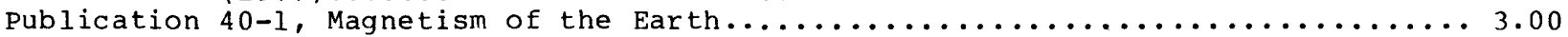

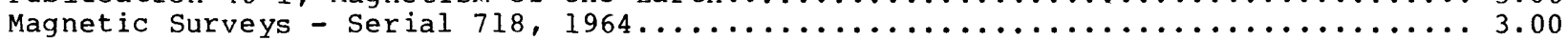

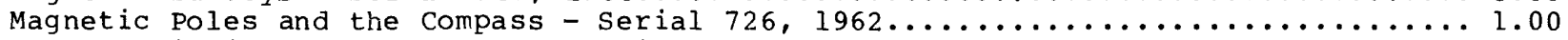
KGRD 2 Bibliography and Index to Literature on Manganese Nodules (1874-1975),

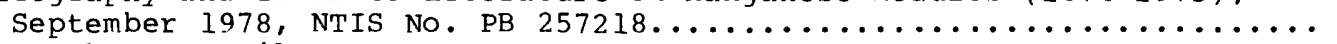

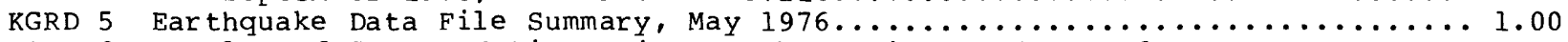

KGRD 6 Catalog of Strong-Motion Seismograph Stations and Records, NTIS No.

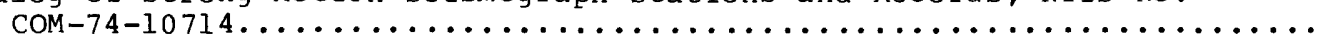

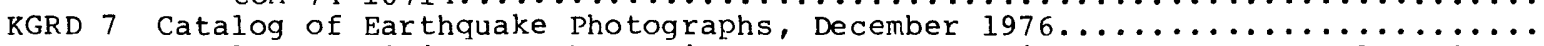

KGRD 8 Catalog of Digital Bathymetric Data for the United States Coastal Regions.

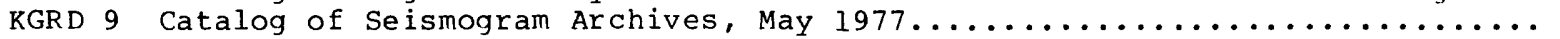

KGRD 10 The Marine Geophysical Data Exchange Format - "MCD $7, \ldots$ September igji....

KGRD 11 Summary of Digital Marine Geophysical Data Holdings (Bathymetric, Gravimetric, and Magnetic Data), september 1978....................

proceedings of Marine Geology Data Management Workshop, May 22-24, 1978........ Free principles underlying the Interpretation of Seismograms, Special Publication

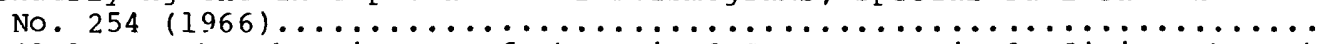

publication $41-1$, Earthquake History of the United states, Revised Edition through

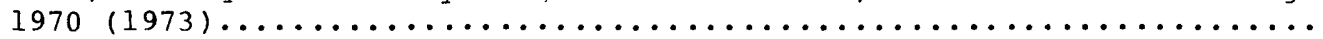

Earthquake History of the United States (1971-76 supplement) $1979 \ldots \ldots \ldots \ldots$ Publication 41-2, Earthquake Investigation in the Western United States, 1931-1964

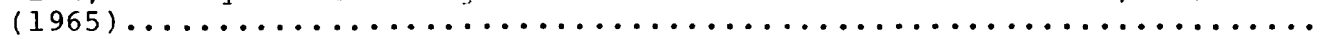

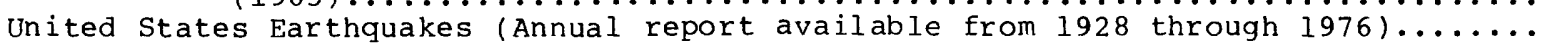


United States Earthquakes, 1977 (Annual report available from 1928)..........

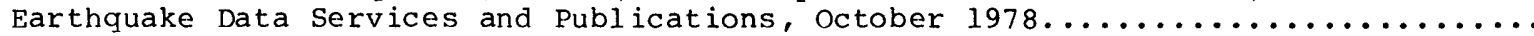

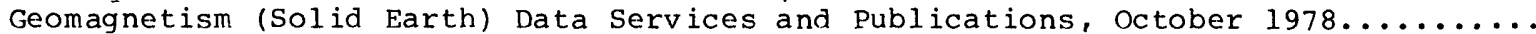
Marine Geology and Geophysics Data Services and Publications, October 1978....... The Puget Sound, Washington Earthquake of April $29,1966$.

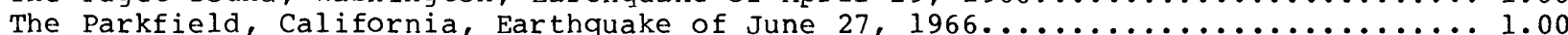

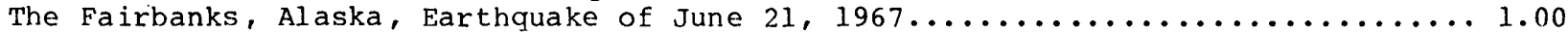

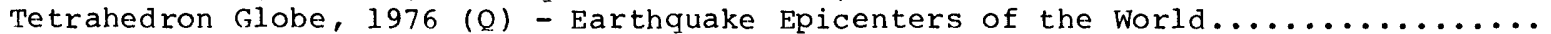

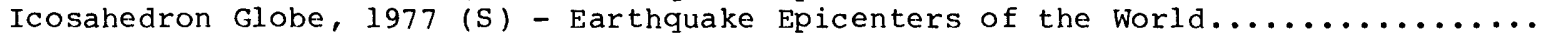

Icosahedron Globe, $1978(\mathrm{~N}-\mathrm{N})$ - International Geomagnetic Reference Field.......

Icosahedron Globe, 1979 (S) - International Geomagnetic Reference Field........

Volcanoes of the World map (L.D. Morris, T. Simkin, and H. Meyers) 1979.........

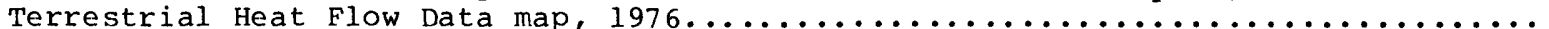

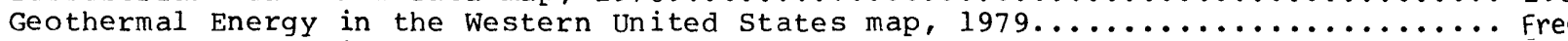

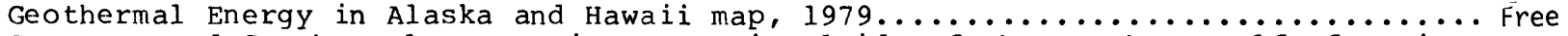

Geopressured-Geothermal Energy in Reservoir Fluids of the Northern Gulf of Mexico

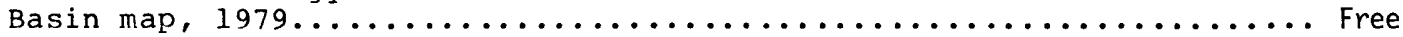

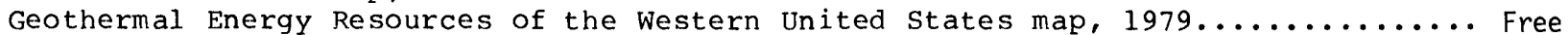

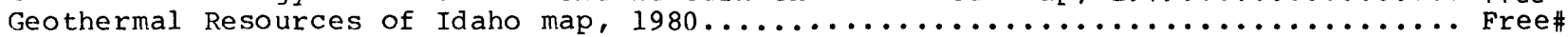

\footnotetext{
**Available from National Technical Information Service (NTIS), 5285 Port Royal Road, Springfield, VA 22161 .

***Price: 10 or more copies, $\$ 0.10$ each; 2 to 9 copies; $\$ 1$ total; 1 copy free.

***Available from Superintendent of Documents, U.S. Government Printing Office, Washington, DC 20402 .

\#\#Available from NOAA, National Ocean Survey, (C44), 6501 Lafayette Ave., Riverdale, MD 20840 .

\#For availability consult NGSDC.
} 